\title{
Growth kinetics and doping of gallium nitride grown by rf-plasma assisted molecular beam epitaxy
}

Aaron Joseph Ptak

West Virginia University

Follow this and additional works at: https://researchrepository.wvu.edu/etd

\section{Recommended Citation}

Ptak, Aaron Joseph, "Growth kinetics and doping of gallium nitride grown by rf-plasma assisted molecular beam epitaxy" (2001). Graduate Theses, Dissertations, and Problem Reports. 1351.

https://researchrepository.wvu.edu/etd/1351

This Dissertation is protected by copyright and/or related rights. It has been brought to you by the The Research Repository @ WVU with permission from the rights-holder(s). You are free to use this Dissertation in any way that is permitted by the copyright and related rights legislation that applies to your use. For other uses you must obtain permission from the rights-holder(s) directly, unless additional rights are indicated by a Creative Commons license in the record and/ or on the work itself. This Dissertation has been accepted for inclusion in WVU Graduate Theses, Dissertations, and Problem Reports collection by an authorized administrator of The Research Repository @ WVU.

For more information, please contact researchrepository@mail.wvu.edu. 


\title{
Growth Kinetics and Doping of Gallium Nitride Grown by rf-Plasma Assisted Molecular Beam Epitaxy
}

\author{
Aaron J. Ptak
}

Dissertation submitted to the Eberly College of Arts and Sciences at West Virginia University

in partial fulfillment of the requirements

for the degree of

Doctor of Philosophy

in

Physics

Thomas H. Myers, Ph.D., Chair

Larry E. Halliburton, Ph.D.

Nancy C. Giles, Ph.D.

Charter D. Stinespring, Ph.D.

Mohindar S. Seehra, Ph.D.

Department of Physics

Morgantown, West Virginia

2001

Keywords:

GaN, Growth Kinetics, Doping, Molecular Beam Epitaxy 


\title{
Abstract \\ Growth Kinetics and Doping of Gallium Nitride Grown by rf-Plasma Assisted Molecular Beam Epitaxy
}

\begin{abstract}
Aaron J. Ptak
A reduced rate for growth of GaN by plasma-assisted molecular beam epitaxy often limits growth to temperatures less than $750^{\circ} \mathrm{C}$, with the reduction significantly larger than thermal decomposition rates. Conditions producing a flux consisting predominantly of either atomic nitrogen or nitrogen metastables have been established using various rf-sources. Atomic nitrogen, possibly coupled with the presence of low energy ions, is associated with the premature decrease in growth rate. An active nitrogen flux consisting primarily of nitrogen metastables produces a temperature dependence more consistent with decomposition rates. Growth with molecular nitrogen metastables results in significantly improved electrical properties.
\end{abstract}

Magnesium incorporation was studied for both (0001), or Ga-polarity and $(000 \overline{1})$, or $\mathrm{N}$-polarity, orientations for various growth conditions. A significant dependence on surface polarity of $\mathrm{Mg}$ incorporation was observed, with up to a factor of twenty-five times more $\mathrm{Mg}$ incorporated on the Ga-polarity. Measurements supported surface accumulation of $\mathrm{Mg}$ during growth, with stable accumulations of close to a monolayer of $\mathrm{Mg}$. $\mathrm{Mg}$ coverage of a monolayer on the Ga-polarity induced a surface polarity inversion. Atomic hydrogen was found to increase the incorporation of $\mathrm{Mg}$ without also incorporating potentially compensating hydrogen.

Beryllium incorporation was also studied for both polarities of $\mathrm{GaN}$. Unlike $\mathrm{Mg}$, surface polarity-related incorporation differences were less pronounced for Be. Measurements also support surface accumulation of Be during growth, with stable accumulations approaching a monolayer for heavier doping levels. Transmission electron microscopy studies indicate the surface layer of Be has a significant effect on 
structure, with severe degradation occurring when accumulation nears monolayer coverage.

High-quality GaN films were grown to study the dependence of controlled oxygen incorporation on polarity and oxygen partial pressure. Oxygen concentrations up to $2.5 \times 10^{22} \mathrm{~cm}^{-3}$ were obtained. About 10 times more oxygen incorporates on $\mathrm{N}$-polar $\mathrm{GaN}$ than on the Ga-polarity in high quality epilayers. Oxygen doping is controllable, reproducible, and uncompensated up to concentrations of at least $10^{18} \mathrm{~cm}^{-3}$ with higher levels showing significant compensation. Layers with oxygen levels above $10^{22} \mathrm{~cm}^{-3}$ exhibit severe cracking. Oxygen incorporation has a weak dependence on $\mathrm{Ga}$ overpressure during Ga-stable growth but dramatically increases for conditions approaching N-stable growth. 
To my wife and my parents. None of this would be possible without you. 
Magnesium stays

On the surface to change

Gallium polar

Like summer to fall

Nitrogen polarity

shuns magnesium

- T.H. Myers 


\section{Acknowledgments}

A work such as this is not possible without help from a great number of resources. The greatest resource I have is my advisor, Dr. Tom Myers. He has done his best to fill this empty vessel with not only knowledge, but the ability to gain more knowledge. For that I am extremely grateful.

I would like to acknowledge the tremendous support I have received from my committee: Dr. Nancy Giles, Dr. Charter Stinespring, Dr. Larry Halliburton, and Dr. Mohindar Seehra. In addition, I would like to thank Dr. Giles and the members of her lab, in particular Dr. Monica Moldovan and Lijun Wang, for all of their work on the

photoluminescence, reflectance, and Raman spectroscopy studies contained in this work. Also, I would like to express my gratitude to Dr. Stinespring and the members of his lab, especially Dr. Katie Ziemer, for all of their work with the mass spectrometry and Auger experiments, and for their general expertise in surface science.

I would also like to recognize Dr. Linda Romano at the Xerox Palo Alto Research Center for TEM measurements and Patrick van Lierde of Evans Analytical Group, Charles Evans and Associates for SIMS characterization.

I want to extend my appreciation to the staff of the Physics Department at West Virginia University, especially Ms. Siobhan Byrne, Ms. Sherry Puskar, Mr. Charles Jackson, and the staff of the Technical Services Unit: Mr. Carl Weber II, Mr. Doug Mathess, Mr. Tom Milam, and Mr. Chuck Sicina.

This work would not have been possible without my colleagues and friends in and out of the Physics Department. The following people have contributed in a significant way to this work through science, friendship, or both: Lauren Hirsch, Matt Millecchia, Brenda VanMil, Dr. Katie Ziemer, Dr. Nelson Garces, Vladimir Stoica, Luke Holbert, Craig Swartz, Liu Ting, Dr. Larry Hornak, Tim Charlton, Carlo Da Cunha, Swati Jain. 
I would not be in the position I am in - indeed, I would not be the person I am without the never-wavering love and support of my family. To my parents: thank you for always believing in me. To my wife: Thank you. Just thank you.

Primary support for this work was provided through the Office of Naval Research, monitored by Dr. C.E.C. Wood. 


\section{Table of Contents}

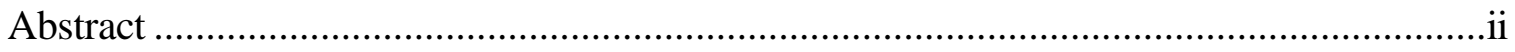

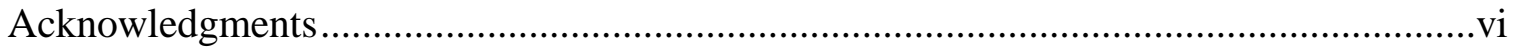

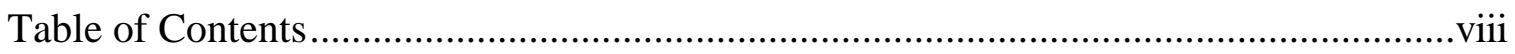

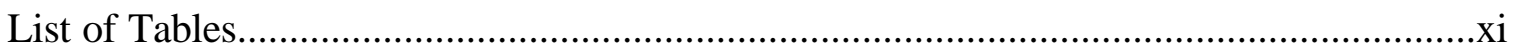

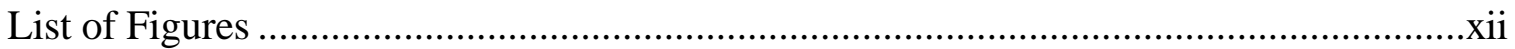

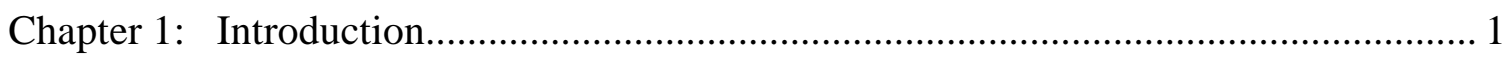

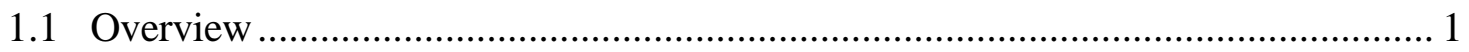

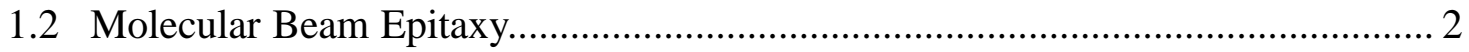

1.3 Basic Properties and Applications of GaN ................................................. 4

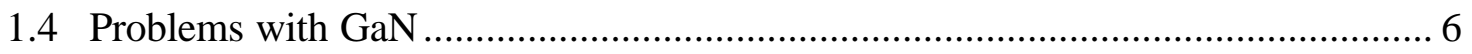

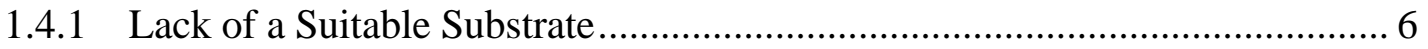

1.4.2 High Background Electron Density....................................................... 7

1.4.3 Ineffectiveness of p-type Doping ........................................................ 7

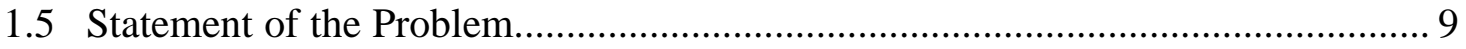

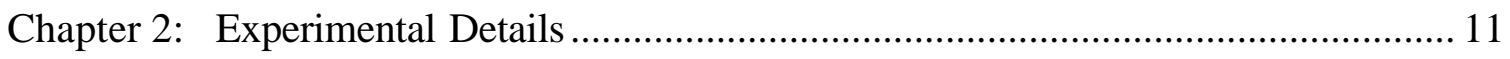

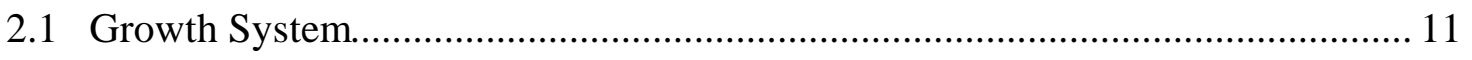

2.1.1 MBE Chamber and Source Flange........................................................ 11

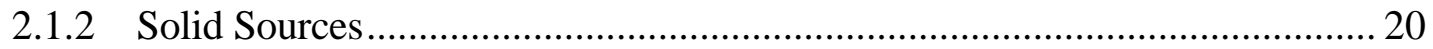

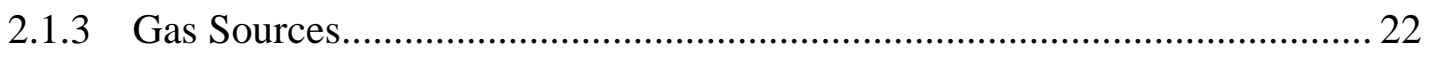

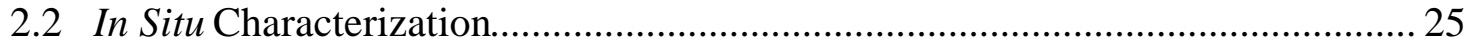

2.2.1 Reflection High Energy Electron Diffraction......................................... 26

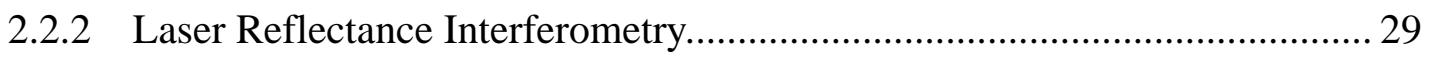

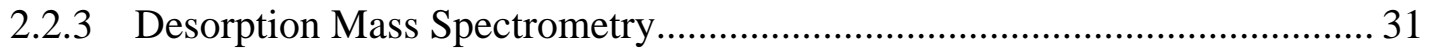

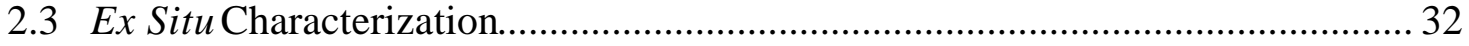

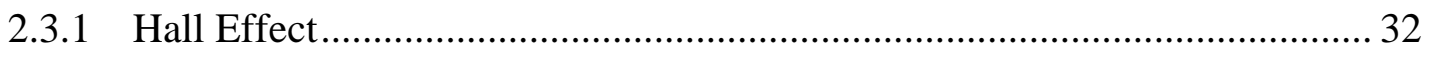

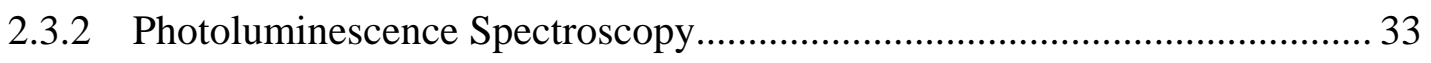

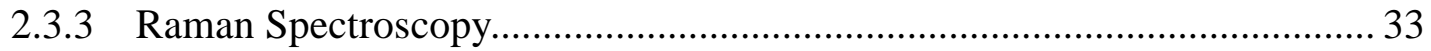

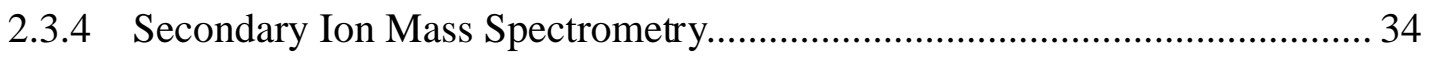

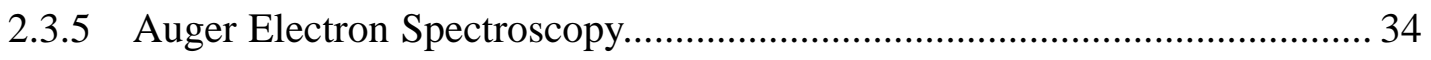

Chapter 3: Nitrogen Source Issues and Growth Kinetics ......................................... 37 


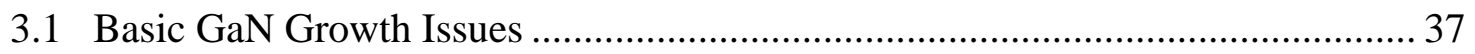

3.1.1 Polarity of the Wurtzite GaN Crystal............................................................ 37

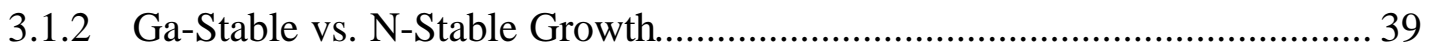

3.2 Determination of Active Nitrogen Species ……………....................................... 40

3.2.1 Description of the Energy Levels of Nitrogen............................................. 44

3.2.2 Description of the rf-Plasma Sources and Experimental Set-up ................... 46

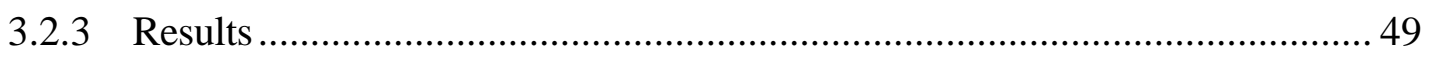

3.3 Determination of the Relative Reactivity of Various Nitrogen Species by the

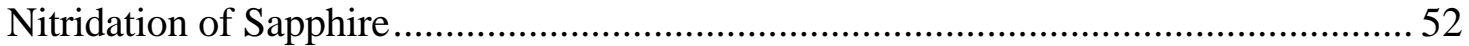

3.4 Effect of Various Nitrogen Species on the Growth of GaN ................................. 63

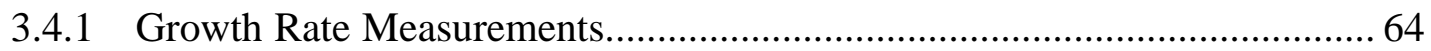

3.4.2 Desorption Mass Spectroscopy Measurements.............................................. 68

3.4.3 Optical and Electrical Measurements............................................................. 68

3.4.4 Effects of Atomic Hydrogen..................................................................... 70

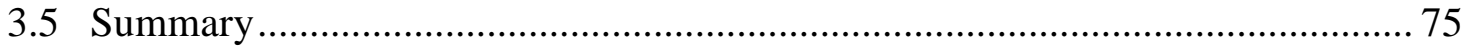

Chapter 4: Magnesium in Gallium Nitride ……………............................................ 77

4.1 Incorporation of Magnesium......................................................................... 78

4.1.1 Growth Conditions ……………………………........................................ 78

4.1.2 Dependence of Magnesium Incorporation on Surface Polarity.................... 79

4.1.3 The Effects of Atomic Hydrogen................................................................. 79

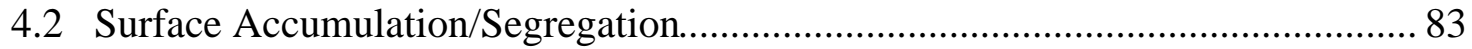

4.2.1 Evidence for Surface Accumulation/Segregation......................................... 83

4.2.2 Surface Rearrangement and Polarity Inversion............................................ 87

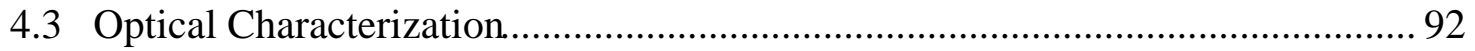

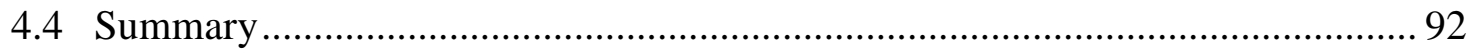

Chapter 5: Beryllium in Gallium Nitride .............................................................. 95

5.1 Incorporation of Beryllium in $\mathrm{GaN}$................................................................... 95

5.1.1 General Behavior of Beryllium Incorporation............................................... 96

5.1.2 Effects of Atomic Hydrogen..................................................................... 99

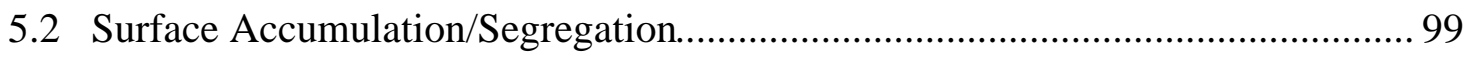

5.2.1 Evidence for Surface Accumulation/Segregation........................................... 99 
5.2.2 Structural Effects of Beryllium Incorporation........................................ 104

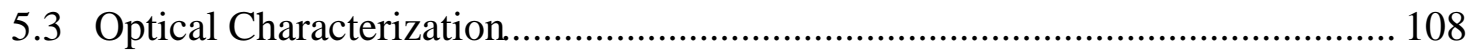

5.4 Electrical Characterization.................................................................... 110

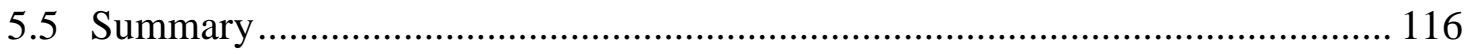

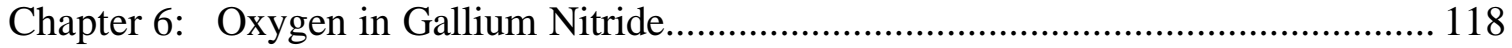

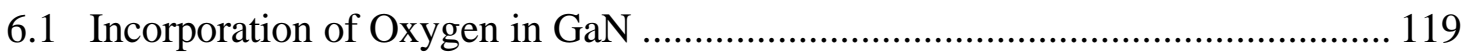

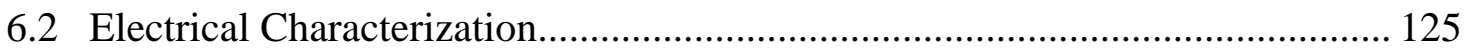

6.3 Structural Effects of Oxygen................................................................... 128

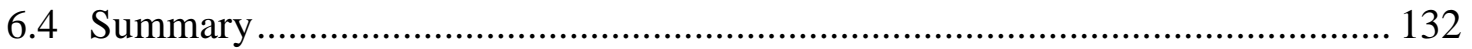

Chapter 7: Conclusions and Work Proposed for the Future .................................... 135

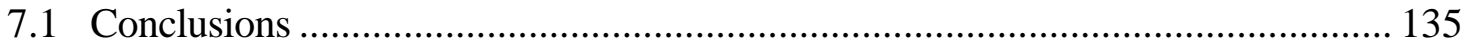

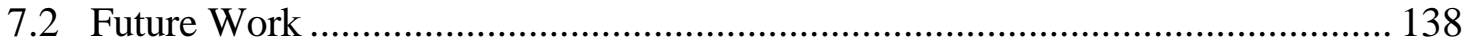

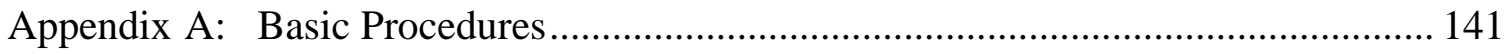

A.1. Sapphire Substrate Preparation ................................................................. 141

A.2. MOCVD- and HVPE-Grown GaN Substrate Preparation............................ 142

A.3. In Situ Substrate Cleaning and Relative Temperature Check ....................... 142

A.4. Buffer Layer Growth................................................................................. 143

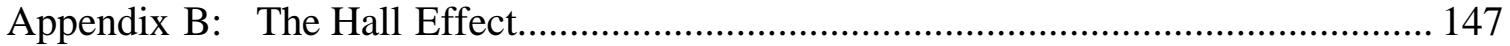

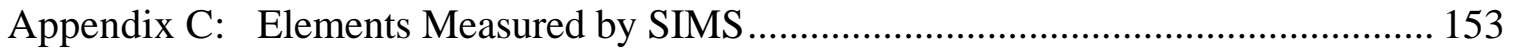

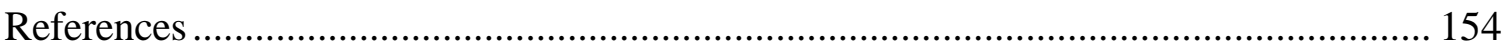




\section{List of Tables}

\section{Chapter 1}

Table 1.1. Properties of wurtzite GaN.

Chapter 3

Table 3.1. Typical flux of ions and atomic nitrogen from the rf-plasma sources, and the actual incorporation rate into GaN. ................................................... 48

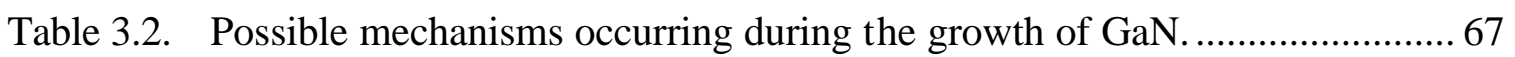

\section{Chapter 4}

Table 4.1 SIMS results for all Mg step-doped samples investigated.

\section{Chapter 5}

Table 5.1 SIMS results for all Be step-doped samples investigated.......................... 97

Table 5.2 Electrical results from Be-doped GaN................................................ 112

\section{Chapter 6}

Table 6.1 The effect of Ga overpressure on oxygen incorporation.......................... 124

Table 6.2 Growth conditions for oxygen doped samples..................................... 130

Table 6.3 Raman spectroscopy and Hall effect results for oxygen-doped samples.... 131 


\section{List of Figures}

\section{Chapter 1}

1.1 Schematic drawing of a typical III-V MBE system...........................

\section{Chapter 2}

2.1 Schematic drawing of the MBE growth system...........................12

2.2 Residual gas analyzer (RGA) scan of the background contamination in the MBE growth system. Note that the only contamination results from trace amounts of hydrogen (2 amu), water (18 amu), nitrogen (28 amu) and carbon dioxide (44

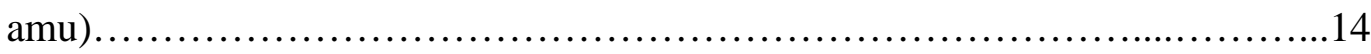

2.3 Schematic drawing of the substrate manipulator/heater design. The thermocouple is kept electrically isolated by being fed through ceramics (hatched regions) until it is in contact with the substrate block ..................................16

2.4 Substrate temperature calibration. Thermocouple reading of the Si/substrate block system, Pyrometer reading of the Si/substrate block system, Al melting point. All calibrations agree when the emissivity change of the substrate block with $\mathrm{GaN}$ coating is taken into account..............................17

2.5 Comparison of the new (top) and old (bottom) source flanges. The new source flange (shown with sources and water cooling lines installed) has nine total ports,

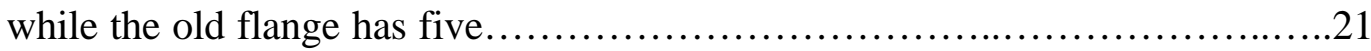

2.6 Vapor pressure curves for gallium, magnesium and beryllium................23

2.7 Schematic drawing of the atomic hydrogen source from Applied EPI, Inc.......24

2.8 Schematic drawing of the RHEED measurement. Shown are the electron gun, the sample, the phosphor screen and the CCD camera........................27

2.9 RHEED diffraction patterns along a [11 $\overline{2} 0]$ azimuth showing a smooth, unreconstructed surface on the left and a two-fold reconstructed surface on the

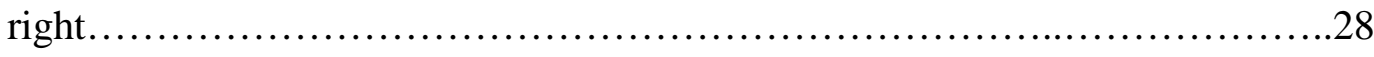

2.10 Laser interferometry growth rate measurement of GaN. The time of 13572 seconds over 5 fringes equates to a growth rate of $\sim 0.18 \mu \mathrm{m} / \mathrm{hr}$ in our system....30 
2.11 A typical Raman measurement of GaN. The position of theE $E_{2}$ peak is used to measure the strain in the sample .35

\section{Chapter 3}

3.1 Ball and stick model of wurtzite GaN. The convention used for the determination of the (0001) direction is indicated, as well as the two other principal symmetry

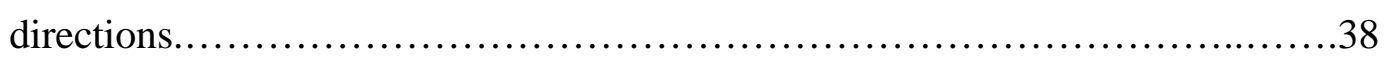

3.2 Typical RHEED images of streaky (left) and spotty (right) patterns.............41

3.3 GaN Growth rate vs Ga flux. The "knee" on the plot for growth without atomic hydrogen represents the transition from a three-dimensional to a two-dimensional growth mode........................................................... 42

3.4 Ga flux necessary to keep the RHEED pattern streaky for both polarities of GaN. Note that the point at $750^{\circ} \mathrm{C}$ for the Ga polarity is only a lower limit as a higher flux could not be reached with the current source...............................43

3.5 Energy levels of molecular nitrogen and the formation energy of GaN. (Courtesy N. Newman, Northwestern University) .................................45

3.6 Schematic drawing of the Oxford CARS-25 rf-plasma source..................47

3.7 Schematic drawing of the experimental set-up used for the characterization of the rf-plasma sources....................................................... 50

3.8 Auger analysis of $\mathrm{Al}, \mathrm{O}, \mathrm{N}$, and $\mathrm{B}$ during nitridation of sapphire using the Oxford source with a single hole aperture. The solid lines are guides to the eyes..........55

3.9 AlN overlayer thickness as a function of time for (a) and , single-hole aperture; (b) and , 9-hole aperture with ions deflected; and (c) , 9-hole aperture without ion deflection. The solid lines are guides to the eye.............56

3.10 Auger analysis of $\mathrm{Al}, \mathrm{O}, \mathrm{N}$, and $\mathrm{B}$ during nitridation of sapphire using the EPI

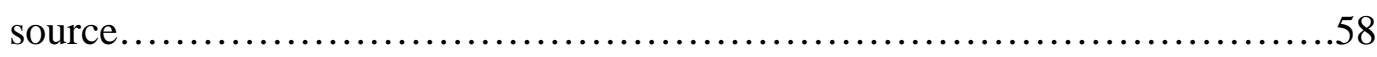

3.11 SIMS measurement of the boron concentration in GaN samples grown by two different rf-plasma sources................................................6 
3.12 Variation of the in-plane lattice constant with nitridation time as observed using RHEED. The $x$-values represent the composition of $\mathrm{Al}_{1-\mathrm{x}} \mathrm{B}_{\mathrm{x}} \mathrm{N}$ that would result in this lattice constant.

3.13 Relative growth rates of $\mathrm{GaN}$ for various conditions. Growth with molecular nitrogen metastables brings the growth rate vs temperature curve more in line with predictions from decomposition rates

3.14 Comparison of the electrical properties of GaN grown with the Oxford and EPI plasma sources. The results for the Oxford source are for samples grown under atomic hydrogen

3.15 Room temperature photoluminescence measurement of undoped $\mathrm{GaN}$ showing a strong free excitonic luminescence signal. This is indicative of high quality material. . .71

3.16 Desorbed Ga flux during growth under atomic hydrogen.....................73

3.17 Effect of atomic hydrogen on the growth rate of GaN.......................74

Chapter 4

4.1 SIMS measurement of $\mathrm{Mg}$ incorporation in a $\mathrm{N}$-polar film grown under various conditions. Shown schematically on the bottom of the figure are the opening and closing positions of the $\mathrm{Mg}$ shutter, as well as the $\mathrm{Mg}$ furnace temperatures......80

4.2 SIMS measurement of Mg incorporation in a Ga-polar film grown under various conditions. Shown schematically on the bottom of the figure are the opening and closing positions of the $\mathrm{Mg}$ shutter, as well as the $\mathrm{Mg}$ furnace temperatures......81

4.3 SIMS measurement of a Mg step-doped layer showing clear signs of surface accumulation. The opening and closing of the Mg shutter is indicated and the substrate temperatures are listed for each peak..................................85

4.4 SIMS measurement of $\mathrm{Mg}$ showing surface accumulation on both polarities of $\mathrm{GaN}$. The region where the $\mathrm{Mg}$ shutter was open is also shown..................86

4.5 SIMS measurement of Mg showing an anomalous decrease in incorporation with a high incident Mg flux. Shown schematically on the bottom of the figure are the opening and closing positions of the $\mathrm{Mg}$ shutter. .88 
4.6 (a) High magnification image of the inversion domain boundary (IDB) taken along the $[11 \overline{2} 0]$ axis with diffraction vector $g=0002$. (b) Lattice image of the IDB taken along the [1 100$]$ axis showing facets that form along the (0001) plane and at an angle $\theta \sim 50$ degrees with the basal plane...........................90

4.7 Convergent beam electron diffraction patterns taken (a) above and (c) below the interface that is indicated by the arrow in (b). (b) is a bright field image taken with a diffraction vector $\mathrm{g}=0002$ near the $[11 \overline{2} 0]$ axis of a $\mathrm{GaN}: \mathrm{Mg}$ film.............91

4.8 Low temperature photoluminescence spectrum of a Mg-doped GaN film. The acceptor bound exciton $\left(\mathrm{A}^{0}, \mathrm{X}\right)$ and donor-acceptor pair (DAP) peaks are

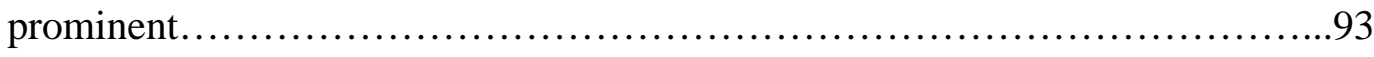

\section{Chapter 5}

5.1 SIMS measurement showing the lack of Be incorporation dependence on the growth conditions of GaN. The shutter openings and closings are shown schematically on the bottom of the figures along with the Be oven temperature

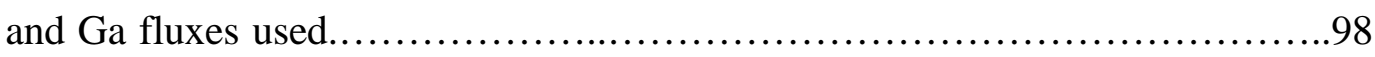

5.2 SIMS measurement of the Be concentration in a Ga-polar GaN sample. The lack of any discernable step-like structure is indicative of surface accumulation 101

5.3 SIMS measurement showing the Be incorporated into a Ga-polar sample. Shown schematically on the bottom of the figure are the opening and closing positions of the Be shutter, as well as the Be furnace temperatures 102

5.4 SIMS measurement inside and just outside the stripe created by the electron irradiation of the RHEED beam. The high-energy electrons appear to suppress the formation of the accumulation layer. Shown schematically on the bottom of the figure are the opening and closing positions of the Be shutter, as well as the Be furnace temperatures

103

5.5 TEM micrographs of the same sample as shown in Figure 5.3. The dislocations bend (left image) upon the first introduction of Be, while high concentrations of Be promote polarity inversion and defect formation (right image). 105 
5.6 High-resolution TEM micrographs of the sample shown in Figure 5.5. The lower left and upper right images show inversion domains and the image at the lower right shows a cubic grain................................................ 107

5.7 Low temperature photoluminescence measurements of Be-doped GaN. Both samples show evidence of a donor-acceptor pair (DAP) at an energy of $3.38 \mathrm{eV}$, while the sample in a) shows the electron-acceptor $\left(\mathrm{e}, \mathrm{A}^{0}\right)$ transition.

5.8 Mobility and carrier concentration vs temperature for a Be-doped GaN layer. The wide scatter of the data represents continuing problems with electrical characterization. 114

5.9 Temperature dependent conductivity of a Be-doped GaN sample. The thermal activation energy is calculated to be $\sim 160 \mathrm{meV}$.

\section{Chapter 6}

6.1 SIMS measurement showing oxygen incorporation in a $\mathrm{N}$-polar step-doped sample. The partial pressure of oxygen used for each step is indicated. The steps were generated by opening and closing a leak valve....

6.2 SIMS measurement showing oxygen incorporation in a Ga-polar step-doped sample. The partial pressure of oxygen used for each step is indicated. The steps were generated by opening and closing a leak valve....

6.3 SIMS of oxygen concentration (lines) and room temperature Hall carrier concentration (points) vs oxygen partial pressure. The lines represent a least-squares fit to SIMS oxygen concentration for constant Ga-overpressure (solid: $1.6 \times 10^{-6}$ Torr BEP, dashed: $1.0 \times 10^{-6}$ Torr BEP). N-polar Hall results were for layers grown under a Ga-overpressure of $1.6 \times 10^{-6}$ Torr BEP while Ga-polar Hall results were for a Ga-overpressure of $1.0 \times 10^{-6}$ Torr BEP.................122

6.4 The variation of oxygen incorporation with Ga overpressure. The Ga BEP is indicated for each step.

6.5 Temperature-dependent Hall effect measurement of an oxygen-doped Ga-polar sample. The line is the fit to the full charge balance equation assuming a single donor with a potential compensating acceptor. The measured donor activation energy is $30 \mathrm{meV}$ and results indicate the sample is uncompensated 
6.6 Ultraviolet fluorescence image of a heavily oxygen doped GaN sample........129

6.7 AFM images from oxygen doped samples. The sample in a) was grown with an oxygen doped buffer layer while the sample in b) had an undoped buffer layer. Note the differences in the height scales (in $\mathrm{nm}$ )

\section{Chapter 7}

7.1 SIMS measurement of a step-doped sample that was co-doped with Be and O. A dramatic increase of oxygen incorporation was observed for high Be fluxes. The partial pressure was held constant during the growth. Be oven temperatures are

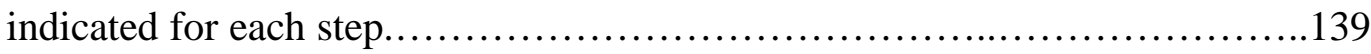

Appendix A

A.1 RHEED images of the buffer layer growth. a) The (11 $\overline{2} 0)$ direction of bare sapphire. b) The (11 $\overline{2} 0)$ direction of GaN after the first minute of growth and the first one minute growth interruption. The pattern is faint and broken. c) After the second growth interruption. d) After the third growth interruption. The pattern is becoming bright and streaky. e) After the fourth growth interruption.

f) The completed buffer layer..................................... 145

\section{Appendix B}

B.1 Schematic for conventional Hall effect measurements.......................148 


\section{Chapter 1: Introduction}

\subsection{Overview}

"Gallic nitride is an exceedingly stable compound." This sentence was printed in a paper by Johnson et al., who first synthesized the compound in 1928. ${ }^{1}$ With these words, research in the nitride semiconductor system began.

Gallium nitride $(\mathrm{GaN})$ along with the other members of the nitride family, aluminum nitride $(\mathrm{AlN})$ and indium nitride $(\mathrm{InN})$, form the basis of an extremely promising system of semiconductors with potential uses in nearly every field imaginable. These three materials form a continuous alloy system of direct band gap semiconductors ranging in energy from $1.9 \mathrm{eV}(\mathrm{InN})$ to $3.4 \mathrm{eV}(\mathrm{GaN})$ to $6.2 \mathrm{eV}(\mathrm{AlN})$ at room temperature. With this range of energies, the III-nitrides can be fashioned into devices that are optically active from red to ultraviolet (uv) wavelengths. This is important as the blue and uv areas of the spectrum are inaccessible with the more familiar arsenide- and phosphide-based semiconductor systems.

An immense amount of effort was expended in the 1960's and 1970's to improve the quality of the nitrides, in particular GaN. Researchers met many obstacles, and were unable to produce material of high quality. Among these difficulties were a high background electron concentration, the lack of a suitable lattice-matched or thermally matched substrate, and the inability to obtain p-type doped material. It was not until the last two decades that the quality of nitride semiconductors really improved with the advent and popularity of such thin film growth techniques as molecular beam epitaxy (MBE) and metalorganic chemical vapor deposition (MOCVD). These growth techniques have been very successful for both the growth of other III-V compounds ${ }^{2}$ and II-VI semiconductors ${ }^{2,3}$ for the production of optical and electronic devices. 


\subsection{Molecular Beam Epitaxy}

The name molecular beam epitaxy first appeared in 1970 and refers to "an epitaxial growth process involving the reaction of one or more thermal beams of atoms or molecules with a crystalline surface under ultra high vacuum conditions." ${ }^{2}$ MBE is related to vacuum evaporation techniques, but distinguishes itself with a much more precise control over growth conditions and fluxes.

MBE systems are based on ultra high vacuum (UHV) chambers that are typically evacuated to less than $10^{-10}$ Torr. A schematic drawing of a typical MBE set-up is shown in Figure 1.1. The UHV environment gives MBE the great advantage of being a very clean process, something that is essential in semiconductor growth where impurities of 10 parts per billion can have a dramatic effect on device performance. Gas-phase interactions are also negligible due to the low operating pressures. During growth by MBE, thermal sources produce beams of atoms or molecules that are directed to the heated substrate where thin film growth occurs. As the name implies, the beams of atoms and molecules are in the molecular, not hydrodynamic, flow regime. The beams can thus be considered as non-interacting, unidirectional sources. This allows for control of the reacting species to be maintained during growth.

MBE is also a low-temperature alternative to growth processes such as MOCVD. MOCVD growth temperatures for $\mathrm{GaN}$ are typically in excess of $1000^{\circ} \mathrm{C}$, while $\mathrm{MBE}$ temperatures are usually in the range of $700-800^{\circ} \mathrm{C}$. This allows for more flexibility in the choice of substrate materials and minimizes thermal effects such as diffusion. Growth rates are typically about $1 / 3 \mu \mathrm{m} / \mathrm{hr}$ (approximately one-third monolayer per second). This allows thickness control on the atomic level. All of these factors combine to help MBE exhibit extremely fine control over semiconductor growth. With this control, MBE has made a tremendous impact on the world of device fabrication. Both optical and electronic devices have been aided greatly by use of the MBE technique. MBE continues to be uniquely suited to the growth of many device structures, such as modulation-doped field-effect transistors (MODFETs) and quantum-well devices among many others. 


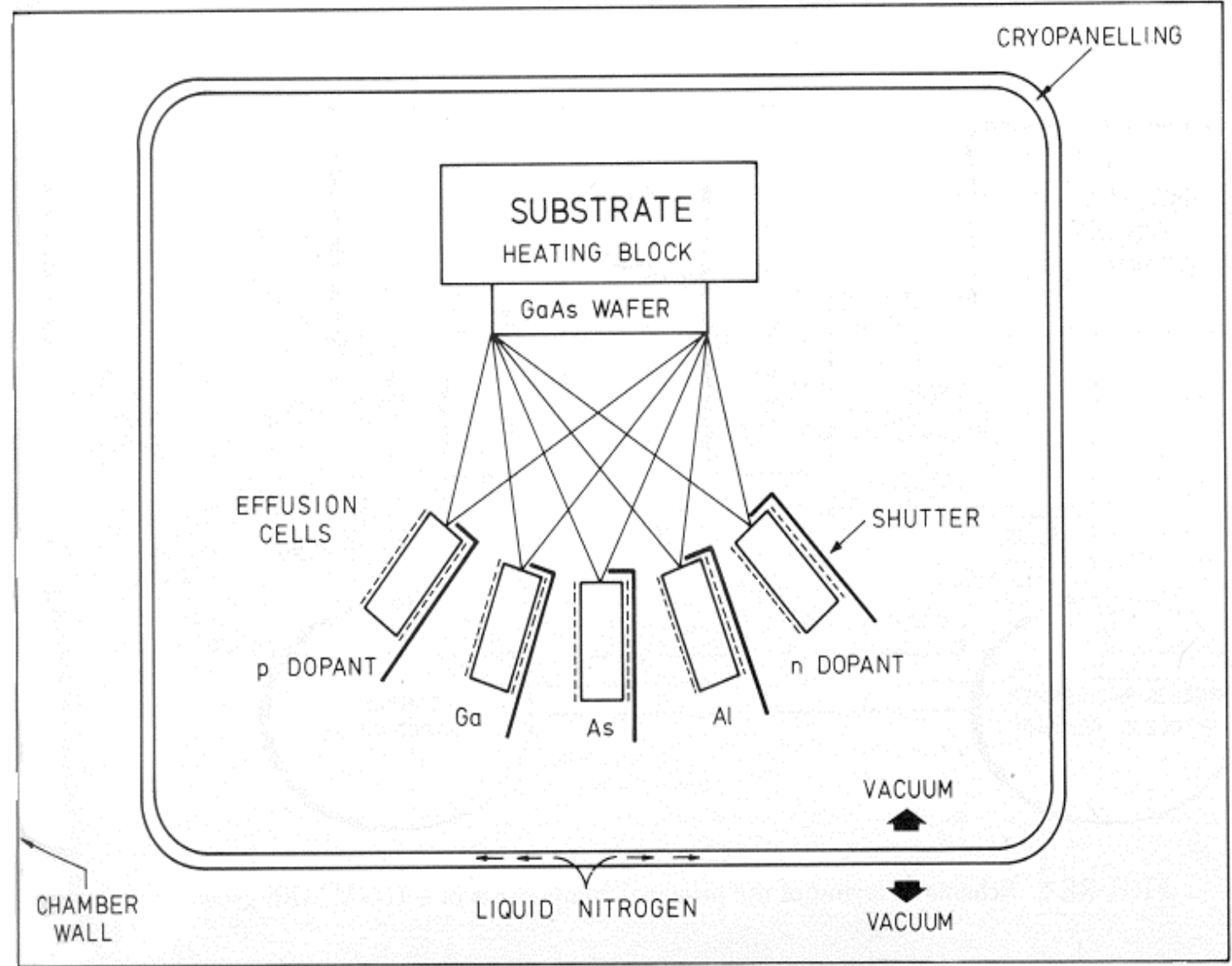

Figure 1.1 Schematic drawing of a typical III-V MBE system. 


\subsection{Basic Properties and Applications of GaN}

Despite the problems during growth with background carriers, lack of a suitable substrate, and inefficient p-type doping, much progress has been made in the development of III-N devices. This is one of the few instances in materials research that useful devices were being produced before a significant understanding of the growth and defect issues was achieved. ${ }^{4} \mathrm{GaN}$ is a very attractive material for devices due to its wide band gap, hardness and high thermal and chemical stability. These characteristics allow the material to be used at elevated temperatures and in caustic environments where the properties of other semiconductor systems would degrade. The knowledge of the basic properties of $\mathrm{GaN}$ has been greatly increased over many years of study. Some of the most important properties are listed in Table 1.1. ${ }^{5}$

Optical devices based on the nitride system are very useful as they are, in principle, able to produce and detect the three primary colors of light: red, green and blue. As such, this alloy system in conjunction with the established arsenide- and phosphidebased systems can be used to create full color displays of amazing clarity. Also, the nitrides will play a crucial role in the field of optical data storage with laser diodes (LDs) being pushed toward shorter and shorter wavelengths. GaN based LDs offer storage with an increased capacity of at least a factor of four since the diffraction-limited storage density increases quadratically with decreasing wavelength. ${ }^{6} \mathrm{GaN}$ is also being used to make light emitting diodes (LEDs), with the first nitride-based device developed by J.I. Pankove in $1971 .{ }^{7}$ In addition to LDs, GaN-based LEDs are now appearing in mainstream markets, notably in the form of bright, long-lasting stoplights and full color displays. The lifetimes of current LEDs based on the nitrides are only limited by the polymer packaging that contains them, and not on the semiconductor material itself. ${ }^{4}$ Future work in this area will concentrate on the improvement of optical output efficiency.

Detectors with a spectral response that stretch into the uv are also of great interest. These "solar-blind" detectors would not be saturated by visible radiation and would be able to cover the 350-200 $\mathrm{nm}$ range by using alloys of GaN and AlN. Such detectors can 
Table 1.1. Properties of wurtzite GaN.

Band gap energy

Band gap temperature coefficient $(\mathrm{T}>180 \mathrm{~K})$

Band gap pressure coefficient $(\mathrm{T}=300 \mathrm{~K})$

Lattice constant $(\mathrm{T}=300 \mathrm{~K})$

Coefficient of thermal expansion $(\mathrm{T}=300 \mathrm{~K})$

Ga site density

Ga surface "site" density

Monolayer thickness

Bilayer thickness

Thermal conductivity

Index of refraction

Dielectric constants

Electron effective mass

Hole effective mass

$$
\begin{aligned}
& E_{g}(300 K)=3.39 \mathrm{eV} \\
& E_{g}(1.6 K)=3.50 \mathrm{eV} \\
& \mathrm{dE}_{\mathrm{g}} /(\mathrm{dT})=-6.0 \times 10^{-4} \mathrm{eV} / \mathrm{K}
\end{aligned}
$$$$
\mathrm{dE}_{\mathrm{g}} /(\mathrm{dP})=4.2 \times 10^{-3} \mathrm{eV} / \mathrm{kbar}
$$

$$
\begin{aligned}
& \mathrm{a}=3.189 \AA \\
& \mathrm{c}=5.185 \AA
\end{aligned}
$$

$$
\Delta \mathrm{a} / \mathrm{a}=5.59 \times 10^{-6} \mathrm{~K}^{-1}
$$$$
\Delta \mathrm{c} / \mathrm{c}=3.17 \times 10^{-6} \mathrm{~K}^{-1}
$$

$\mathrm{N}_{\mathrm{Ga}}=4.28 \times 10^{22} \mathrm{~cm}^{-3}$

$\mathrm{N}_{\text {surf }}=1.1 \times 10^{15} \mathrm{~cm}^{-2}$

$2.59 \AA$ (along c-axis)

$5.18 \AA$ (along c-axis)

$\kappa=1.3 \mathrm{~W} / \mathrm{cm} \mathrm{K}$

$\mathrm{n}(1.00 \mathrm{eV})=2.33$

$\mathrm{n}(3.38 \mathrm{eV})=2.67$

$\varepsilon_{0}=8.9$

$\varepsilon=5.35$

$\mathrm{m}_{\mathrm{e}}^{*}=0.20 \pm 0.02 \mathrm{~m}_{0}$

$\mathrm{m}_{\mathrm{h}}{ }^{*}=0.8 \pm 0.2 \mathrm{~m}_{0}$ 
be used to examine high temperature flames or detect missile plumes in the atmosphere without interference from the sun or other visible light sources. ${ }^{4}$

Electronic devices based on the nitrides are also being actively developed. GaN possesses a high electron saturation velocity and a large breakdown voltage. When combined with an excellent thermal conductivity, GaN becomes a very promising material for high power, high frequency applications where traditional $\mathrm{Si}$ and $\mathrm{SiGe}$ technologies fail. ${ }^{8,9,10}$

\subsection{Problems with GaN}

While nitride devices have enjoyed much success, many of the basic problems that plagued early researchers still remain. It is necessary to find permanent solutions so that nitride device technology may continue to prosper.

\subsubsection{Lack of a Suitable Substrate}

One of the largest stumbling blocks in GaN thin film growth has been the lack of bulk GaN substrates for homoepitaxy. Researchers have been forced to use alternative substrates since there is not yet a mature technology for the bulk synthesis of $\mathrm{GaN}$. Unfortunately, there is no substrate available that is physically and thermally matched to $\mathrm{GaN}$. Hence, GaN has been grown heteroepitaxially on a wide variety of substrates and orientations, including but not limited to, $\mathrm{Si}, \mathrm{GaAs}, \mathrm{NaCl}, \mathrm{GaP}, \mathrm{InP}, \mathrm{SiC}, \mathrm{W}, \mathrm{ZnO}$, $\mathrm{MgAl}_{2} \mathrm{O}_{4}, \mathrm{TiO}_{2}, \mathrm{MgO}, \mathrm{LiAlO}_{2}$, and $\mathrm{Al}_{2} \mathrm{O}_{3}$. Sapphire, $\mathrm{Al}_{2} \mathrm{O}_{3}$, has been the most common substrate in use due to its wide availability, ease of cleaning and handling, and stability at high temperatures.

The largest drawback for the use of sapphire is the $16 \%$ lattice mismatch with GaN. Even if one considers the scheme where six lattice constants of GaN would fit onto seven lattice constants of the oxygen sublattice of sapphire, the resulting mismatch is still $0.5 \%$. This is a considerable barrier to overcome. In addition to the lattice mismatch, there is a mismatch in thermal expansion coefficients of $\sim 29 \%$. This indicates that even if $\mathrm{GaN}$ grows strain-free at the growth temperature, the result of cooling the sample 
would induce a large amount of strain in the material. SiC substrates have become a popular alternative to sapphire for GaN growth as high-quality substrates are now more available and affordable. Although $\mathrm{SiC}$ displays a lattice mismatch of $\sim 3.4 \%$ the thermal mismatch is slightly better than sapphire. $\mathrm{SiC}$ has the added benefit of being intentionally doped, allowing backside contacts that will eliminate at least one step during post-growth processing. GaN film quality will continue to suffer until bulk GaN substrates become available.

\subsubsection{High Background Electron Density}

Until recently, most unintentionally doped GaN had a high n-type background carrier concentration, often as high as $10^{20} \mathrm{~cm}^{-3}$. Many groups have brought this background down to less than $10^{15} \mathrm{~cm}^{-3}$ in recent years, but there have been no reports of high resistivity uncompensated GaN films.

The origin of this high n-type background has been blamed both on nitrogen vacancies and residual oxygen impurities in the samples. There exists a mounting body of work in support of oxygen contamination as the cause of the high n-type background. Oxygen can originate in the growth system in many ways: as an impurity in the $\mathrm{NH}_{3}$ precursor in MOCVD; as a residual water background in MBE; from dissociation of the sapphire substrate; or from leaching of the quartz tube in many plasma sources. Regardless of the cause, the n-type background can still play a role in many studies. The background concentration of electrons can also have a detrimental effect on p-type doping. The residual electrons need to be overcompensated for p-type conduction to occur. This overcompensation has been achieved for the most part by taking more care in the purification of source materials and by improving vacuum conditions.

\subsubsection{Ineffectiveness of p-type Doping}

It is typically easy to make a wide band gap semiconductor either n-type or p-type, but not both. N-type doping is relatively easy in $\mathrm{GaN}$ as seen from the high residual n-type background. Although compensation by the residual electron 
concentration has become much less of an issue, p-type doping has presented many problems.

One set of candidates for p-type dopants in III-V materials are the Group IV elements $\mathrm{C}, \mathrm{Si}$, and $\mathrm{Ge}$. For p-type doping, these elements substitute for a $\mathrm{N}$ atom resulting in an excess of conducting holes. Unfortunately, Si and Ge have been shown to be n-type dopants sitting on the $\mathrm{Ga}$ site, while $\mathrm{C}$ is amphoteric and is now used to produce high-resistivity material. ${ }^{11}$ The Group II elements are also obvious choices for p-type dopants. However, it has been proposed that elements with a d-shell of electrons will form a resonance with the lower valence bands of the crystal and will form a deeper level in the valence band. ${ }^{12}$ This has been experimentally confirmed with $\mathrm{Zn}$ and $\mathrm{Ca}$, both of which have a d-shell of electrons and form levels much deeper than that expected from the simple hydrogenic model of impurities. Neither $\mathrm{Mg}$ nor Be have a d-shell and both have been shown to form relatively shallow levels in GaN.

$\mathrm{Mg}$ is currently the p-type dopant of choice, even though the acceptor energy level of $\mathrm{Mg}$ in $\mathrm{GaN}$ is $\sim 200 \mathrm{meV} .{ }^{13}$ Room temperature hole concentrations greater than about $10^{18} \mathrm{~cm}^{-3}$ have been achieved, ${ }^{14}$ but $\mathrm{Mg}$ has several problems. Only $\sim 1 \%$ of the $\mathrm{Mg}$ atoms will be electrically active at room temperature due to the high activation energy. The large concentration of ionized and neutral impurities present for necessary doping levels decreases hole mobilities drastically. Mobilities ranging from $1-10 \mathrm{~cm}^{2} / \mathrm{V}$-sec are typical, limiting the achievable conductivity. The lack of p-type conduction leads to high resistivity contacts that are the limiting factor in many devices. The p-type layer accounts for nearly the entire voltage drop in a GaN-based laser diode leading to high turn-on voltages and severely limiting the device lifetime. Other problems include the necessity of post-growth processing to activate the $\mathrm{Mg}$ that is compensated during growth with hydrogen. This activation can be achieved either by low-energy electron beam irradiation (LEEBI), ${ }^{15}$ or thermal annealing. ${ }^{16} \mathrm{Mg}$ also shows a severe memory effect during growth by MOCVD leading to broad doping profiles in addition to other problems. Tantalizing evidence has indicated that $\mathrm{Be}$ may be a shallower dopant than $\mathrm{Mg}$, but Be also suffers from what may be severe limitations on its 
effectiveness. $\mathrm{Mg}$ and $\mathrm{Be}$ will be discussed in more detail in Chapters 4 and 5, respectively.

\subsection{Statement of the Problem}

$\mathrm{GaN}$ is certainly well suited to a virtually endless number of applications, but problems do exist. The aim of this work is to further the understanding of basic growth and doping issues in GaN. The following questions are addressed:

1. The active nitrogen generated by different rf-plasma sources is generally a complex mixture of molecules, atoms, ions and other excited species. Is it possible by studying different rf-plasma sources to control the dominant type of active specie emitted from the source? What is the relative reactivity of each active specie? What effect does each specie have on the growth of GaN, such as the dependence of growth rate on substrate temperature or for growth under an atomic hydrogen flux?

2. As alluded to above, p-type doping suffers from many problems. What can be learned by studying the incorporation of $\mathrm{Mg}$ in $\mathrm{GaN}$ ? How is incorporation affected by growth on the two different polarities of GaN? What can be determined about the role of surface accumulation/segregation effects that have been seen in the literature? Can anomalous reports of p-type conduction at high incident $\mathrm{Mg}$ flux be explained by these results? How does growth under atomic hydrogen affect the incorporation of $\mathrm{Mg}$ ?

3. A possible alternative to $\mathrm{Mg}$ as a p-type dopant in $\mathrm{GaN}$ is Be. Few studies of $\mathrm{Be}$ in $\mathrm{GaN}$ have been performed and almost nothing is known about its incorporation characteristics. How does Be incorporate in GaN? How does polarity affect this incorporation and how does this compare to $\mathrm{Mg}$ ? What is the effect of atomic hydrogen on the incorporation and electrical activation of Be? Is Be a suitable acceptor impurity for use in GaN? What can optical and 
electrical characterizations determine about Be as an acceptor? How does the incorporation of Be affect the structure of GaN?

4. Few careful studies of intentional oxygen doping in $\mathrm{GaN}$ have been performed and no unambiguous studies have been used to determine the role of crystal polarity on incorporation. How do the two polarities of $\mathrm{GaN}$ affect the incorporation of oxygen? What is the reason for any observed difference? How does oxygen behave as a donor in GaN? What are the effects of oxygen doping on the structure of $\mathrm{GaN}$ for both low and high concentrations? 


\section{Chapter 2: Experimental Details}

\subsection{Growth System}

Molecular beam epitaxy is a UHV growth technique that has been shown to be of great usefulness over the last two or three decades in the fabrication of, among other things, semiconductor devices. MBE has a number of features that make it an attractive option for the growth of semiconductors. Among these is the use of a UHV environment that enables a very high degree of control over background impurities. Gas-phase interactions are also negligible in a UHV environment, while they can play a significant role in such alternative growth methods as MOCVD and hydride vapor phase epitaxy (HVPE). Other favorable characteristics include the lower temperatures involved in the growth process, as well as the avoidance of unintentional hydrogen incorporation during growth. Hydrogen can play a strong role in semiconductors, as will be seen in this work by the intentional inclusion of hydrogen during some growths, and cannot be avoided in MOCVD and HVPE.

\subsubsection{MBE Chamber and Source Flange}

A new MBE system was used in this work that was designed specifically for the study of III-N compounds such as InN, GaN and their solid solutions. The previous system used for the growth of $\mathrm{GaN}$ was much larger than the current system, was not optimized for GaN growth, and contained contaminants generated by II-VI compound semiconductor growth. The substrate heater on the previous system was not capable of the temperatures needed to grow high quality $\mathrm{GaN}$ (approximately $800^{\circ} \mathrm{C}$ ). Also, a spring-loaded type $\mathrm{K}$ thermocouple was used that could not survive the high temperatures for very long. After continued heating, the spring system that held the thermocouple in contact with the substrate block would fail, and there was no way to know when this occurred. Consequently, any previous temperature calibration would be suspect. This, when combined with the degradation of the type $\mathrm{K}$ thermocouple itself, resulted in a continual uncertainty in the actual substrate temperature. The current MBE system, shown schematically in Figure 2.1, was constructed in order to satisfy several 


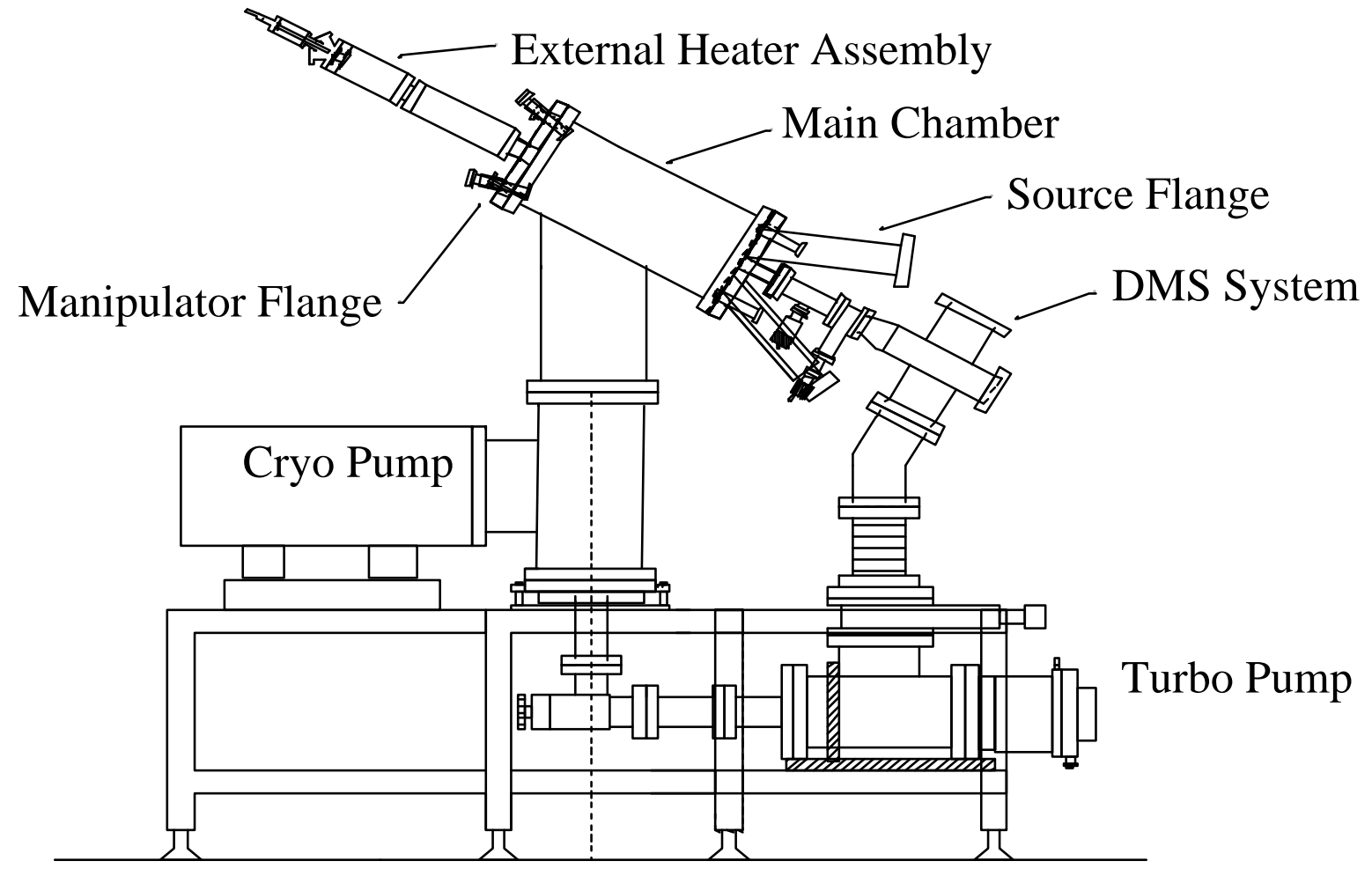

Figure 2.1 Schematic drawing of the MBE growth system. 
requirements. These were: a smaller source-to-substrate distance allowing an increase in both flux at the substrate and in growth rate; a redesigned substrate heater and thermocouple assembly; the incorporation of the same in situ diagnostics as the previous system; and the capability for desorption mass spectrometry (DMS). The redesigned substrate heater is capable of temperatures in excess of $1000^{\circ} \mathrm{C}$. The source-to-substrate distance is on the order of $20 \mathrm{~cm}$, allowing for high source fluxes at the substrate and growth rates for GaN greater than $2 \mu \mathrm{m} / \mathrm{hr}$. DMS and more familiar diagnostics such as reflection high-energy electron diffraction (RHEED) and laser reflectance interferometry (see later in this chapter) are also present. A final point is that with a new system, there is no contamination from unwanted impurities. It has been proposed that some effects seen during the growth of $\mathrm{GaN}$ are the result of such impurities. An example is the appearance of a $2 \times 2$ surface reconstruction that has been used by many as an indicator of optimal growth conditions. ${ }^{17,18}$ This reconstruction, however, likely results from the adsorption of unwanted arsenic present in the system from previous studies involving arsenic containing compounds. ${ }^{19}$ No substances besides $\mathrm{N}, \mathrm{Ga}, \mathrm{In}, \mathrm{Sn}, \mathrm{Be}, \mathrm{Mg}$, and $\mathrm{O}$, have ever been used in this system. Consistent with Smith et al. ${ }^{19}$ who also work in a system free of arsenic, this reconstruction has never been observed in this system despite a concentrated search indicating that it is not due to an intrinsic effect. The current growth chamber is pumped with a CTI-8 cryogenic pump with a nominal pumping speed of $1000 \mathrm{l} / \mathrm{s}$ and has a typical base pressure of $5 \times 10^{-11}$ Torr, consisting mainly of trace amounts of nitrogen, hydrogen and water. Figure 2.2 shows a typical background mass spectrum. The design and initial construction phases for the new MBE system are described in great detail elsewhere. ${ }^{20}$

Absolute values of substrate temperature are one of the most difficult things to measure during thin film growth. A large amount of effort was invested to be as accurate as possible in the determination of substrate temperatures. A new approach was used for the placement of the monitoring thermocouple and for determining its position. Knowledge of the substrate temperature involved several complementary calibrations. 


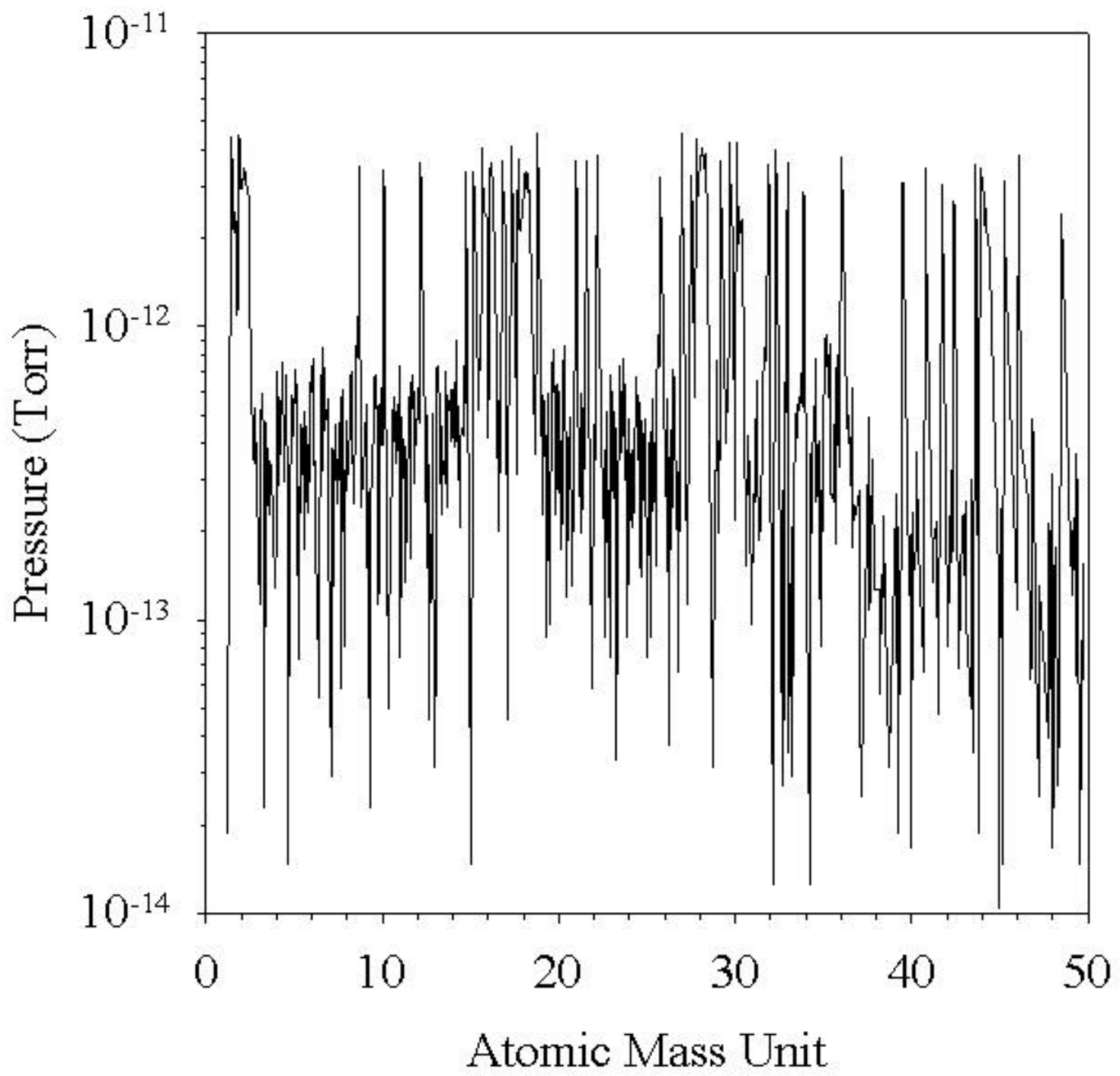

Figure 2.2 Residual gas analyzer (RGA) scan of the background contamination in the MBE growth system. Note that the only contamination results from trace amounts of hydrogen (2 amu), water (18 amu), nitrogen (28 amu) and carbon dioxide (44 amu). 
A schematic of the substrate manipulator/temperature monitoring stage of the MBE system is shown in Figure 2.3. A type $\mathrm{C}$ thermocouple is in physical contact with the back of the molybdenum substrate block. This type of thermocouple is designed for use with much higher temperatures than the type $\mathrm{K}$ thermocouple in the previous system. The type $\mathrm{C}$ thermocouple is electrically isolated by the use of ceramics as it is fed through the heater. A linear motion mini-thimble with a maximum range of \pm 0.63 " adjusts the position of the thermocouple. When the thermocouple is moved forward into contact with the substrate block, electrical continuity is established. In this way, it is clear that the monitoring thermocouple is in contact with the substrate block during growth.

Three different methods were used to calibrate the substrate temperature. First, an additional thermocouple was mounted through a small hole into the face of the substrate block. This allowed a comparison of the temperatures at the front and back surfaces of the block. Figure 2.4 shows the data generated by monitoring both thermocouples while raising the substrate temperature. These measurements were taken with a piece of silicon mounted on the substrate block with molten indium to simulate the conditions of growth. While the temperature is being raised, the front thermocouple parallels the dial temperature, but is lower by about $15^{\circ} \mathrm{C}$. Due to contact with the molten In, the $\mathrm{Si}$ is at virtually the same temperature as the block. When the Si loses thermal contact with the block, i.e. when the In evaporates, the difference in temperature becomes much greater. This is consistent with a change in emissivity due to the lack of In. When the In is present, the block temperature tracks the dial temperature because the In (with a low emissivity) does not radiate much heat away. When the In evaporates, the Si is no longer in thermal contact and the emissivity of the system is defined by the substrate block. Since the block is much less shiny, more heat is radiated away and the difference in temperature becomes greater. It is necessary to work in a regime where the molten metal does not evaporate to avoid this problem and obtain results that are consistent with growth conditions. 


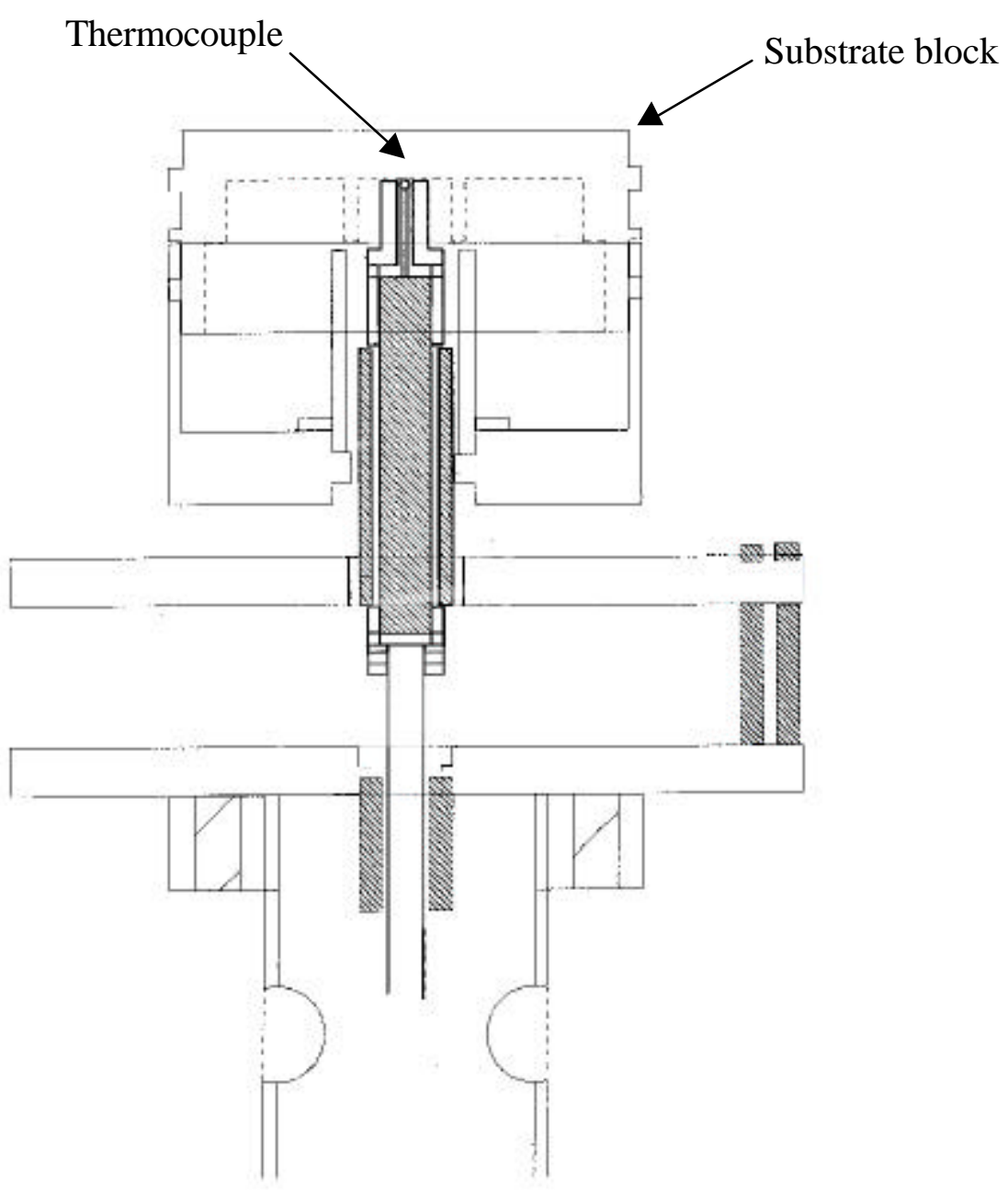

Figure 2.3 Schematic drawing of the substrate manipulator/heater design. The thermocouple is kept electrically isolated by being fed through ceramics (hatched regions) until it is in contact with the substrate block. 


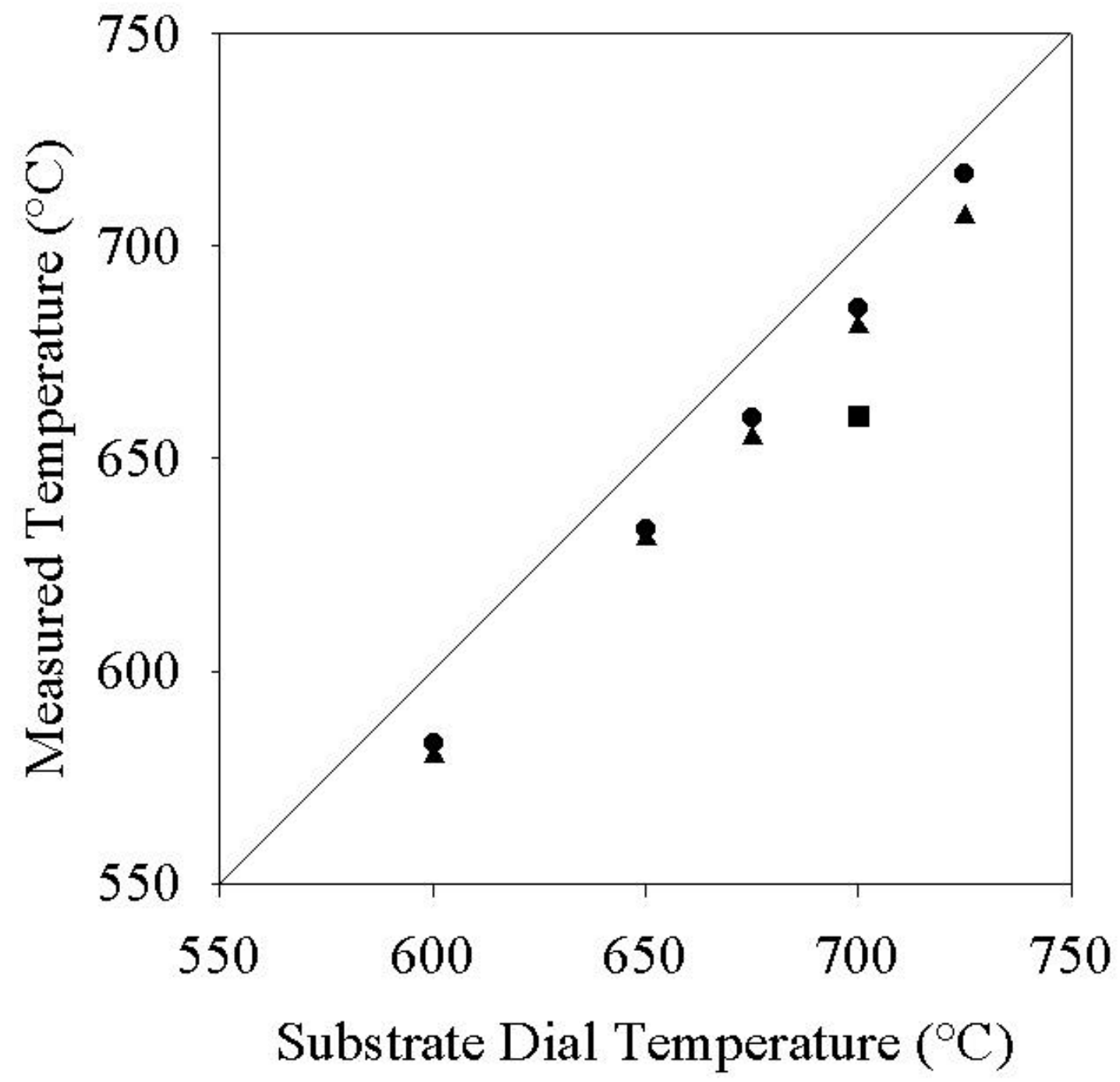

Figure 2. 4 Substrate temperature calibration. Thermocouple reading of the $\mathrm{Si} /$ substrate block system, Pyrometer reading of the Si/substrate block system, $\mathrm{Al}$ melting point. All calibrations agree when the emissivity change of the substrate block with GaN coating is taken into account. 
Second, optical pyrometry measurements were made using relative emissivity values for silicon determined at WVU. The emissivity values for $\mathrm{Si}$ were determined in the laboratory of Prof. Larry E. Halliburton using a Si wafer mounted to a molybdenum block with molten indium. The $\mathrm{Si}$ was heated in a high temperature tube furnace. A type $\mathrm{K}$ thermocouple was placed in a small hole in the back of the molybdenum block and was assumed to be at the same temperature as the $\mathrm{Si}$. This is a reasonable assumption since the furnace should form an isothermal cavity. An OS-3000 AS high temperature optical pyrometer (Omega Engineering, Inc., Stamford, CT) with a spectral range from 2.1-2.3 $\mu \mathrm{m}$ was used to monitor infrared radiation coming from the $\mathrm{Si}$. The pyrometer compares the intensity of this infrared radiation with a stored value based on a blackbody spectrum to determine the temperature. A standard MBE optical port was placed between the pyrometer and the Si to simulate the substrate temperature calibration conditions as accurately as possible. As such, these measurements determine the relative values for the emissivity of the $\mathrm{Si} / \mathrm{In} /$ molybdenum block composite system. The relative emissivity of this composite system could be determined by inputting the proper emissivity value into the programmable pyrometer such that the pyrometer and thermocouple readings were the same. The emissivity values determined in this way were approximately linear in the temperature region investigated, with a range of values from 0.68 to 0.64 for temperatures from 550 to $730^{\circ} \mathrm{C}$, respectively. For a temperature of $700^{\circ} \mathrm{C}$ an uncertainty in the emissivity of 0.01 leads to a change in temperature of less than $2^{\circ} \mathrm{C}$.

The relative emissivity values were used to measure the temperature of the $\mathrm{Si}$ for the calibration of substrate temperature. The pyrometer readings, also shown in Figure 2.4, are quite close to those of the thermocouple mounted in the face of the block while using these measured emissivity values. The average deviation between the two measurements is approximately $5^{\circ} \mathrm{C}$.

As a third, independent calibration, Al dots were sputtered onto a piece of sapphire to use the melting point of $\mathrm{Al}$ as a temperature calibration. The Alon-sapphire 
sample was heated in the chamber while the optical reflectance was measured with the laser reflectance set-up used for growth rate measurements (see Section 2.2.2). When the $\mathrm{Al}$ dots melt at $660^{\circ} \mathrm{C}$, the reflectance of the $\mathrm{Al}$ changes. The dial temperature at which this happens gives an absolute data point for temperature calibration. This point is also shown in Figure 2.4 and is seen to be lower than the pyrometer and thermocouple calibrations by $\sim 25^{\circ} \mathrm{C}$. One complication with these calibrations results from the change in emissivity of the block due to coating with GaN during growth. When the block is clean and free of any deposited GaN, the emissivity is low and the block can be up to $30^{\circ} \mathrm{C}$ hotter for a given substrate dial temperature than if the block is covered with GaN. This change in temperature is confirmed with a measurement of the Ga condensation temperature on sapphire. This is a relative temperature measurement allowing for the day-to-day changes of substrate temperature to be taken into account. Details of this measurement are found in Appendix A. The calibration performed with the melting of Al dots was done with a coated block, while the other calibrations were done with a clean block. Based on numerous relative calibrations, this $30^{\circ} \mathrm{C}$ difference must be taken into account. When this is done, all of the calibrations agree to within $10^{\circ} \mathrm{C}$ and it is seen that there is an approximate $45^{\circ} \mathrm{C}$ difference between the real temperature and the dial temperature in the region of interest for standard growth conditions with a coated block. Due to the extensive temperature calibrations, all temperatures presented in this work are accurate to within $\pm 10^{\circ} \mathrm{C}$. Note that after the block is cleaned, a "sacrificial" growth was performed to re-coat the block to allow known conditions for growth.

The system's original source flange allowed the simultaneous use of two gas sources (nitrogen and hydrogen) as well as two solid sources (typically gallium and a dopant furnace). There was also the capacity for DMS in line-of-sight with the substrate. There were no optical ports on this source flange to be used for laser reflectance (and hence growth rate measurements), although this could be accomplished via two non-ideal ports located elsewhere on the system. As studies became more complex, it was necessary to augment the system's capabilities by designing a new source flange. For example, the windows used for reflectance measurements utilized an additional reflection from a non-mirrorlike surface that drastically reduced signal strength. In addition, during 
measurements, these windows became coated with indium also reducing signal. This meant that growth rate measurements could not be continued for very many growths before the system would have to be opened and the windows replaced. There were also problems with the operation of the Ga furnace. The standard furnace has a diameter of 1.47 in., while the port in which it resided was only 1.50 in. in diameter. During continued operation, excess Ga that was reflected back toward the furnace when the shutter was closed would both build up around the furnace causing a thermal short and wicking of $\mathrm{Ga}$ inside the furnace radiation shielding. This severely limited the lifetime of a new furnace and caused the need for it to be rebuilt after only months of operation. In addition to the cost of replacing the furnace itself, the premature failure also meant that the system must be opened with the additional cost of time and material. Furthermore, a general rule of thumb in MBE is to maximize the capabilities of the system whenever possible. Although the above mentioned problems were certainly factors, the major driving force for redesigning the source flange was the necessity of expanding growth capabilities with the inclusion of additional source ports. The new source flange has room for two gas sources, four solid sources (including two dopant sources and larger diameter ports for the high temperature furnaces), a line of sight port to be used as an additional dopant port or continuing DMS studies, and two optical ports for laser reflectance. The two source flanges are compared in Figure 2.5. To date, there has been no additional thermal shorting of the Ga furnace in the new source flange.

\subsubsection{Solid Sources}

Molecular beams are typically generated using standard MBE-compatible Knudsen cells consisting of a resistive heater assembly surrounding a crucible containing the source material with a thermocouple to monitor the source temperature. Temperature stability to within $0.5^{\circ} \mathrm{C}$ is achieved with a feedback loop and a Eurotherm 818 temperature controller. The temperature of the source and the vapor pressure of the material determine the flux of material that reaches the substrate. The vapor pressure 

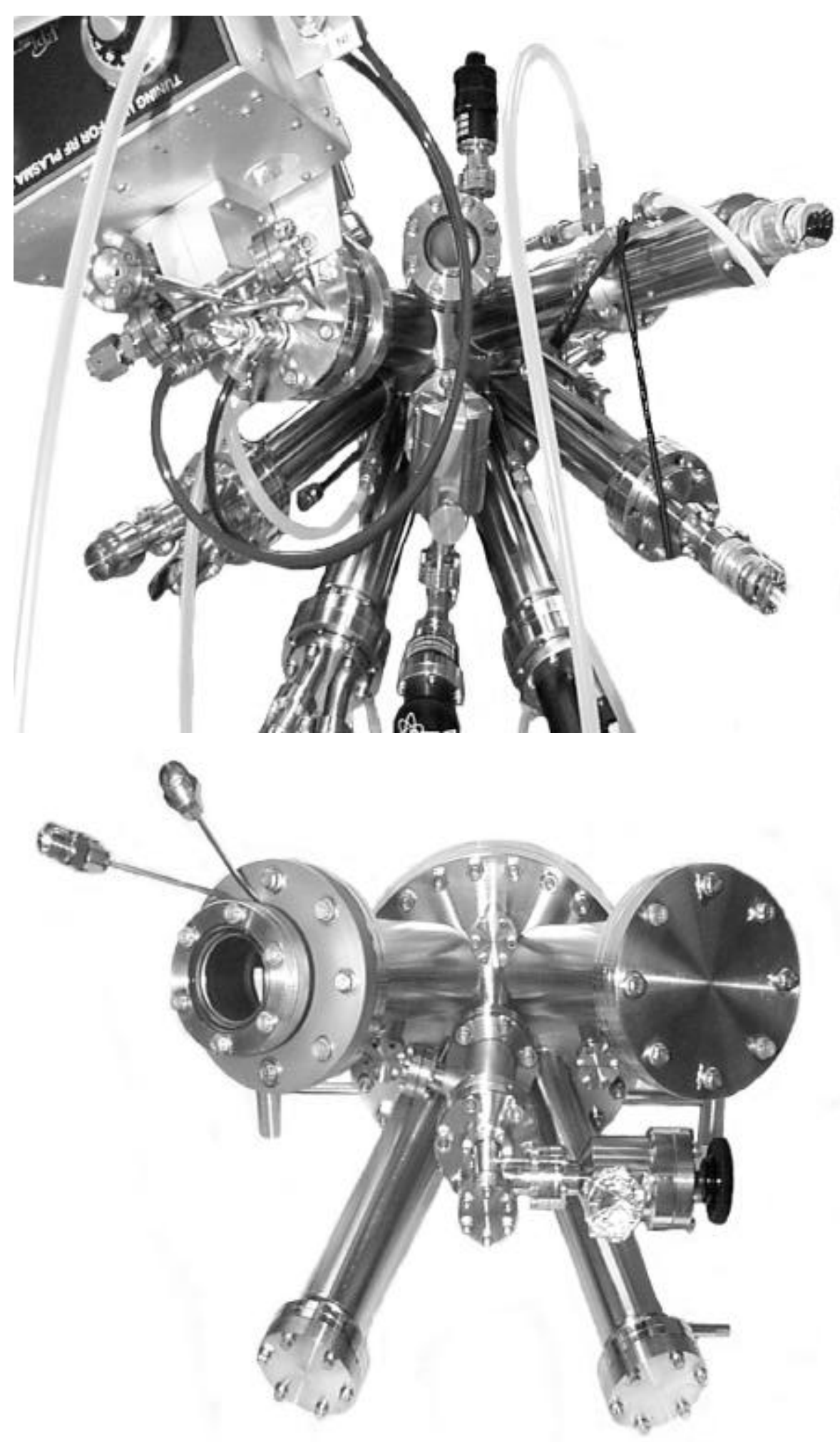

Figure 2.5 Comparison of the new (top) and old (bottom) source flanges. The new source flange (shown with sources and water cooling lines installed) has nine total ports, while the old flange has five. 
curves for $\mathrm{Ga}, \mathrm{Be}$, and $\mathrm{Mg}$ are shown in Figure 2.6. An EPI-40-M modified filament cell is used to produce the $\mathrm{Ga}$, a low-temperature EPI-40 furnace is used for the $\mathrm{Mg}$ flux, while a special EPI-5-D dopant furnace is used for Be. The $\mathrm{Mg}$ furnace is equipped with a type $\mathrm{K}$ thermocouple, while the others contain thermocouples that are type $\mathrm{C}$. The modified, so called "hot lip" cell provides additional heating at the exit orifice to prevent Ga droplet condensation at the edge of the crucible. This material could fall back into the molten Ga causing material spitting.

\subsubsection{Gas Sources}

Three different types of gas sources were used in this work: a thermal cracker for hydrogen, a gas inlet valve for oxygen, and an rf-plasma source for active nitrogen.

The hydrogen source (EPI-AHS, Applied-EPI, St. Paul, MN) operates by the dissociation of molecular hydrogen into hydrogen atoms. Ultra high purity hydrogen (99.9999\%) flows into the source (shown schematically in Figure 2.7) through a UHV leak valve and across a tungsten filament that is heated using a DC current to a temperature greater than $1600^{\circ} \mathrm{C}$. Some fraction of the molecular hydrogen is dissociated by a catalytic reaction on the hot tungsten surface resulting in a mixture of atomic and molecular hydrogen being produced by this source. The following parameters were used for all samples in this study unless otherwise noted: 9.5A, 400-450W (which corresponds to a temperature of approximately $2200^{\circ} \mathrm{C}$ and an estimated atomic hydrogen "cracking" efficiency of 5-10\%), ${ }^{21}$ and system beam equivalent pressure (BEP) of $2 \times 10^{-6}$ Torr for hydrogen. The BEP is the pressure read directly on the ion gauge that is calibrated for nitrogen gas. Based on the reported cracking efficiency along with a relative ion gauge sensitivity of $0.5,^{22}$ we can find the actual flux of $\mathrm{H}$ atoms. We estimate that $2 \times 10^{-6}$ Torr BEP corresponds to a flux of approximately 1-2 monolayer/s equivalent of atomic hydrogen at the growth surface.

The source for oxygen doping experiments consisted of a 3/16 in. stainless steel tube connected to a source port that stuck through a $1 / 4 \mathrm{in}$. hole in the source flange at normal incidence to the substrate. Ultra high purity oxygen $(99.998 \%)$ was connected to 


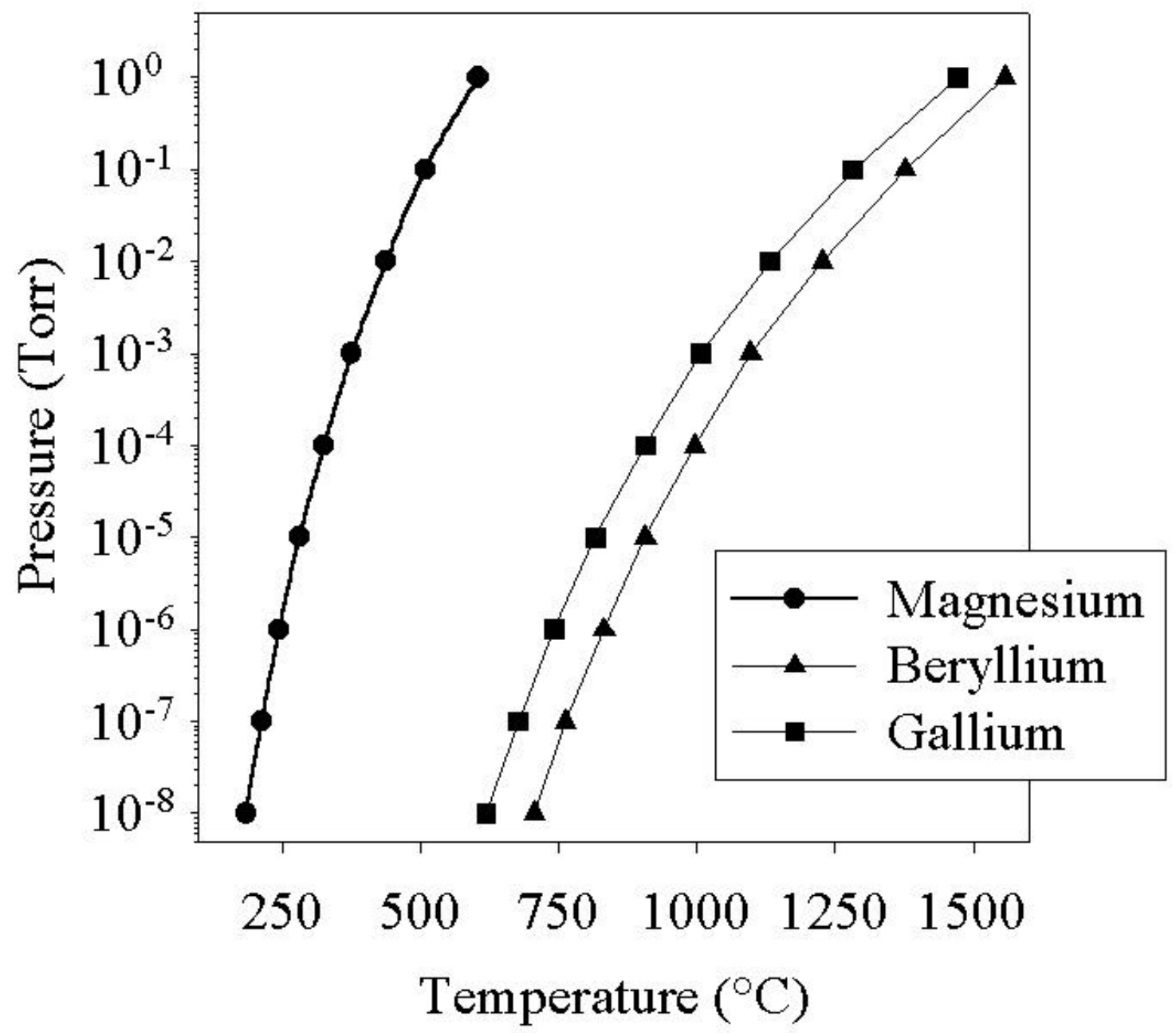

Figure 2.6 Vapor pressure data (from Ref. 20) for Mg Be, and Ga. 


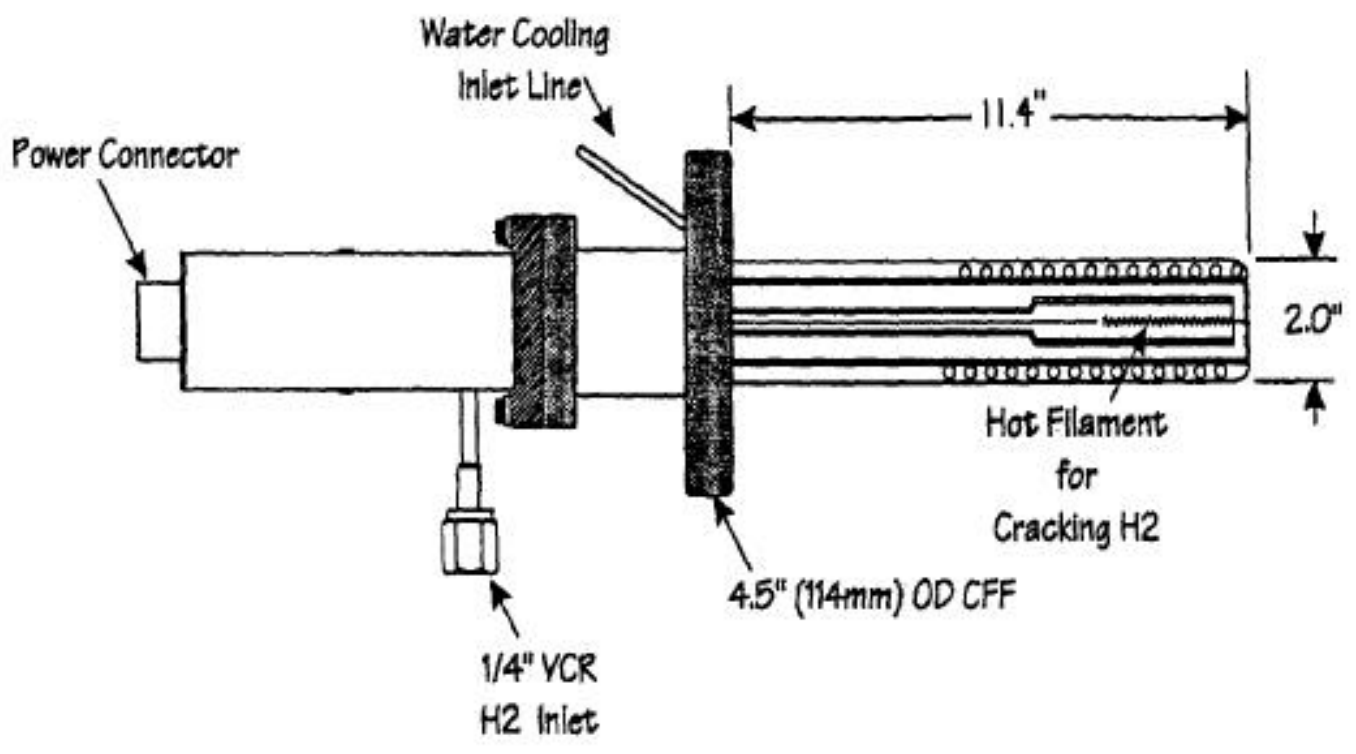

Figure 2.7 Schematic drawing of the atomic hydrogen source from Applied EPI, Inc. 
the tube through a UHV leak valve. Connections were in place to allow pre-pumping of the gas lines before the introduction of oxygen to minimize contamination. An RGA-200 residual gas analyzer from Stanford Research Systems (Sunnyvale, CA) was used to monitor partial pressures of oxygen introduced into the growth chamber.

The source of activated nitrogen is perhaps the single most important piece of equipment to consider for the growth of $\mathrm{GaN}$, as the type of source chosen can have a dramatic impact on the material grown (see Chapter 3). It is necessary to "activate" molecular nitrogen, as the binding energy of $\mathrm{N}_{2}(9.5 \mathrm{eV})$ is too high to thermally dissociate at the substrate temperatures used. One possibility is the use of ammonia, $\mathrm{NH}_{3}$, which will dissociate at these temperatures and provide a source of nitrogen for growth. It also, however, provides a large reservoir of hydrogen that will influence the growth kinetics. Another possibility is the use of an ECR-plasma source, but this type of source is known to produce large quantities of high-energy ions that create crystalline damage.

Our choice was an rf-plasma source, which can produce significant active nitrogen with smaller amounts of ions that also typically have small energies. Even this choice is complicated as the specifics of the design of the rf-plasma source produces a different mix of activated nitrogen, including atoms, ions, metastables and a background of molecular nitrogen. All samples in this study were grown using an EPI Unibulb rf-plasma source with the exception of a limited number of undoped growths outlined in Chapter 3 that use an Oxford CARS-25 rf-plasma source. Both of these sources are described in detail in Chapter 3.

\subsection{In Situ Characterization}

As stated earlier, the UHV nature of the MBE technique has a number of advantages. Among these is the availability of in situ characterizations that allow the determination of material parameters without disturbing the growth process. 


\subsubsection{Reflection High Energy Electron Diffraction}

Perhaps the most widely used in situ technique in MBE is that of reflection highenergy electron diffraction (RHEED). RHEED is commonly used only as a qualitative measure of surface perfection although it is a powerful tool that allows for quantitative analysis of the crystal structure such as the determination of lattice constants, growth rates and surface reconstructions.

The RHEED measurement is simple and robust. A standard set-up is shown schematically in Figure 2.8. A VE-026 electron gun (Vieetech Japan Co., Ltd.) produces a beam of energetic electrons (in this study, $25 \mu \mathrm{A}$ at $13 \mathrm{keV}$ ) that strike the sample surface at a grazing incidence $\left(1-2^{\circ}\right)$ and produce a diffraction pattern on a phosphor screen on the opposite side of the growth chamber. A charge-coupled device (CCD) camera captures the screen image for storage and later analysis. Because the RHEED beam is aligned nearly in the same plane as the substrate, the electron gun and camera do not interfere with the valuable real-estate used by sources and detectors that need to be closer to the substrate normal.

Due to the grazing incidence of the beam the electron momentum normal to the surface is kept small, making RHEED a very surface-sensitive technique that only samples the top few monolayers of the film. This allows the state of the sample surface to be probed during growth, presumably without affecting the sample. A variety of diffraction patterns can be seen using RHEED. These depend on, among other things, the crystal symmetry, the surface morphology, and any surface reconstruction that may be present. For example, if the diffraction pattern is made up of long, uniform streaks, the surface is growing smoothly, in a two-dimensional fashion. If, however, the diffraction appears in the form of spots, the surface is more three-dimensional and rough. The appearance of a ring-like pattern is usually an indication that a sample is polycrystalline. RHEED patterns from GaN, one showing a streaky, unreconstructed surface and one showing a surface that is two-fold reconstructed, are shown in Figure 2.9. Both images 


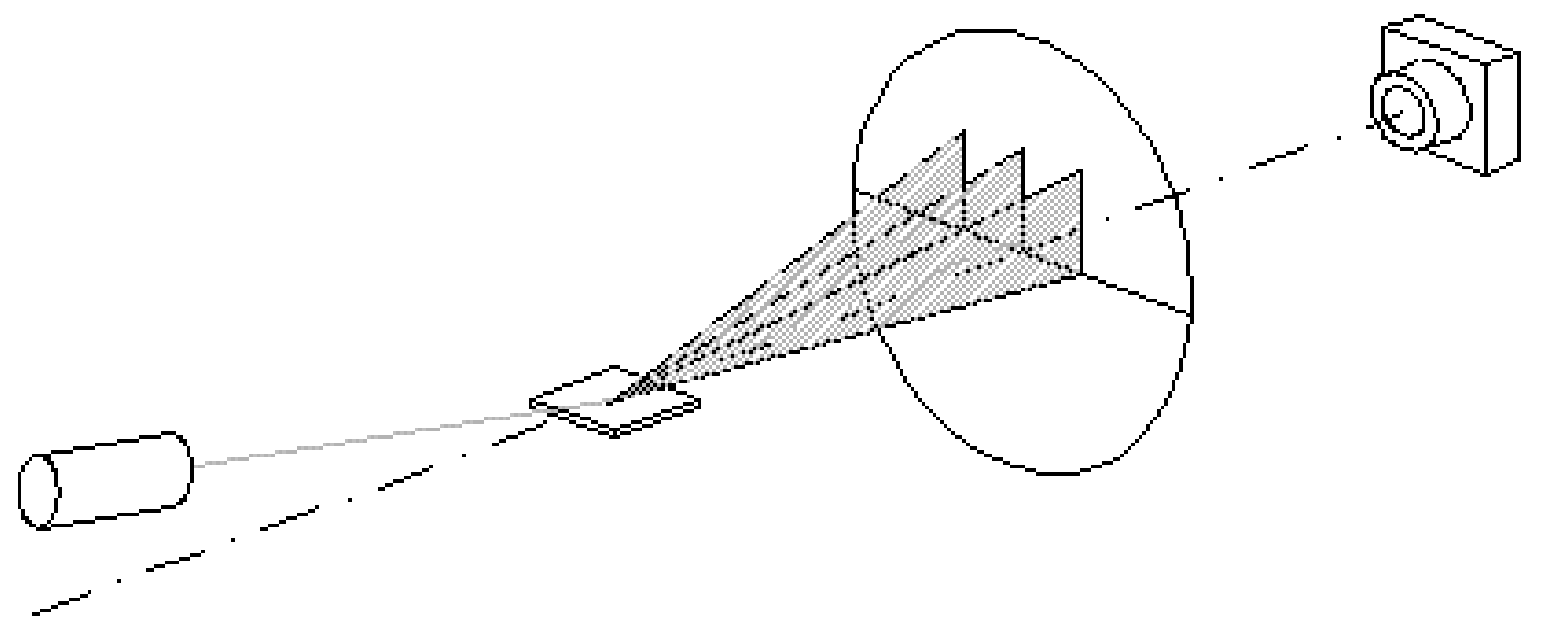

Figure 2.8 Schematic drawing of the RHEED measurement. Shown are the electron gun, the sample, the phosphor screen and the CCD camera. 


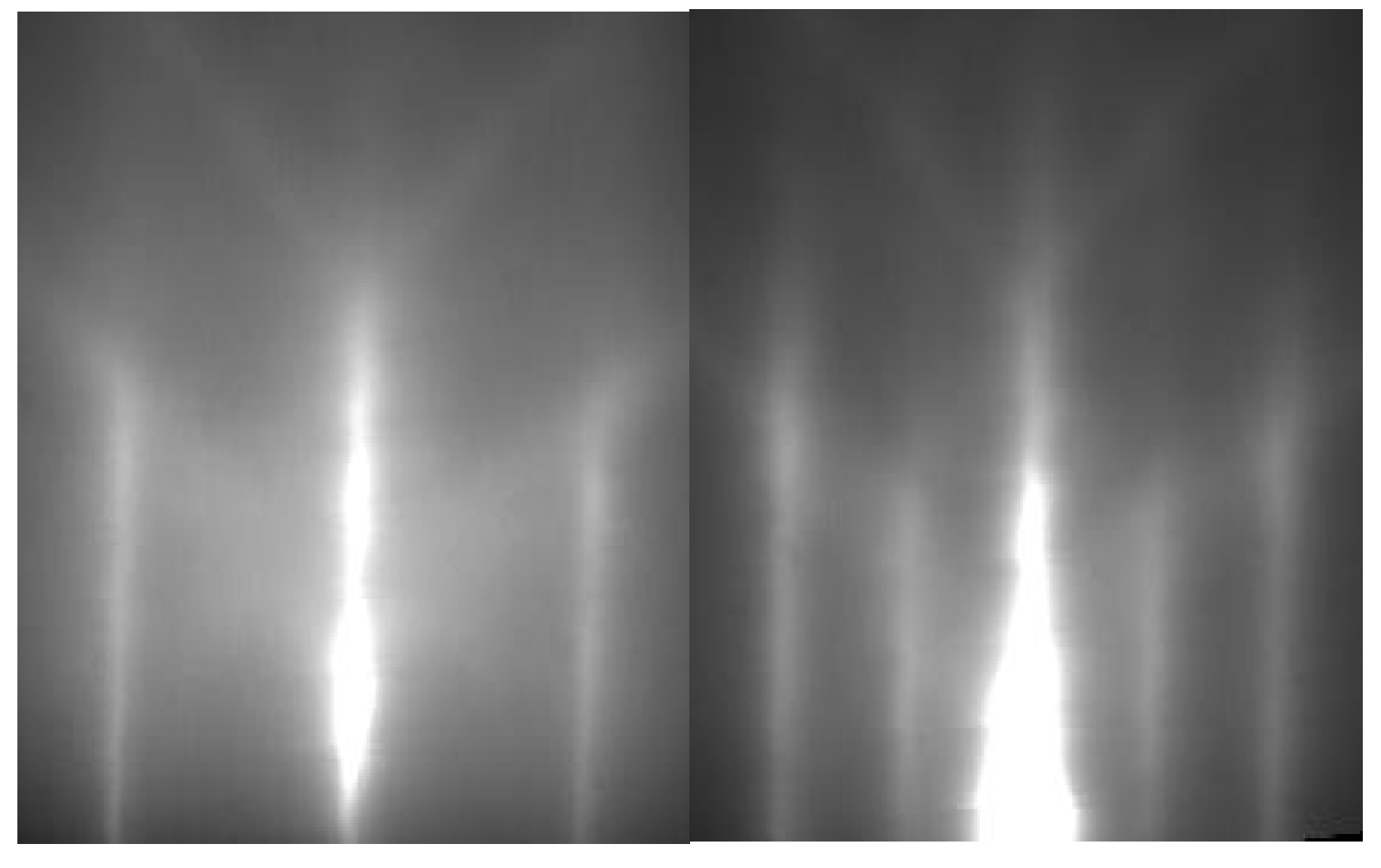

Figure 2. 9 RHEED diffraction patterns along a [11̄20] azimuth showing a smooth, unreconstructed surface on the left and a two-fold reconstructed surface on the right. 
are taken along the $[11 \overline{2} 0]$ direction. Numerous atomic force microscopy (AFM), scanning tunneling microscopy (STM) and transmission electron microscopy (TEM) studies in the III-N system have been used to correlate RHEED patterns and surface morphologies. ${ }^{23,24}$

\subsubsection{Laser Reflectance Interferometry}

It is very convenient to be able to determine the growth rate of the sample while it is growing. This allows the effect of a single parameter on the growth rate to be determined while keeping all other parameters identical. For example, either the effects of atomic hydrogen or the effect of varying substrate temperature on the growth rate could be determined for many conditions in one growth, as will be discussed in the next chapter. In situ determination of growth rate is also necessary, as will be seen in Chapters 4 and 5, in order to correctly determine the timing of such events as the opening or closing of a dopant shutter. The growth rate measurement involves a pair of viewports, each facing the substrate at an equal angle. An S1031 5-mW red diode laser (Thorlabs, Inc., Newton, NJ) with a wavelength of $680 \mathrm{~nm}$ is directed toward the sample through one viewport and the reflected light is collected at the opposing viewport with a photodiode. The laser is electrically modulated by use of a square-wave function generator allowing the use of lock-in techniques to eliminate noise from the surrounding environment. An SR850 DSP Lock-In Amplifier (Stanford Research Systems, Sunnyvale, CA) is used to collect interference spectra such as the one shown in Figure 2.10. As the thickness of the film changes (i.e. as the film grows), the thin film interference condition for the monochromatic red light sweeps through maxima and minima. Reference samples are grown with a known number of interference fringes (an interference maximum to the next maximum is one fringe, see Figure 2.10), and the thicknesses of these samples are measured ex situ using a standard optical transmittance measurement on a Cary 14 spectrophotometer. The thicknesses determined in this way have been found to agree with TEM measurements. Given the thickness and the number

of fringes for each of these reference samples, a "growth rate constant" can be found. In the current system, one fringe represents approximately $0.133 \mu \mathrm{m}$ of $\mathrm{GaN}$ growth. This 


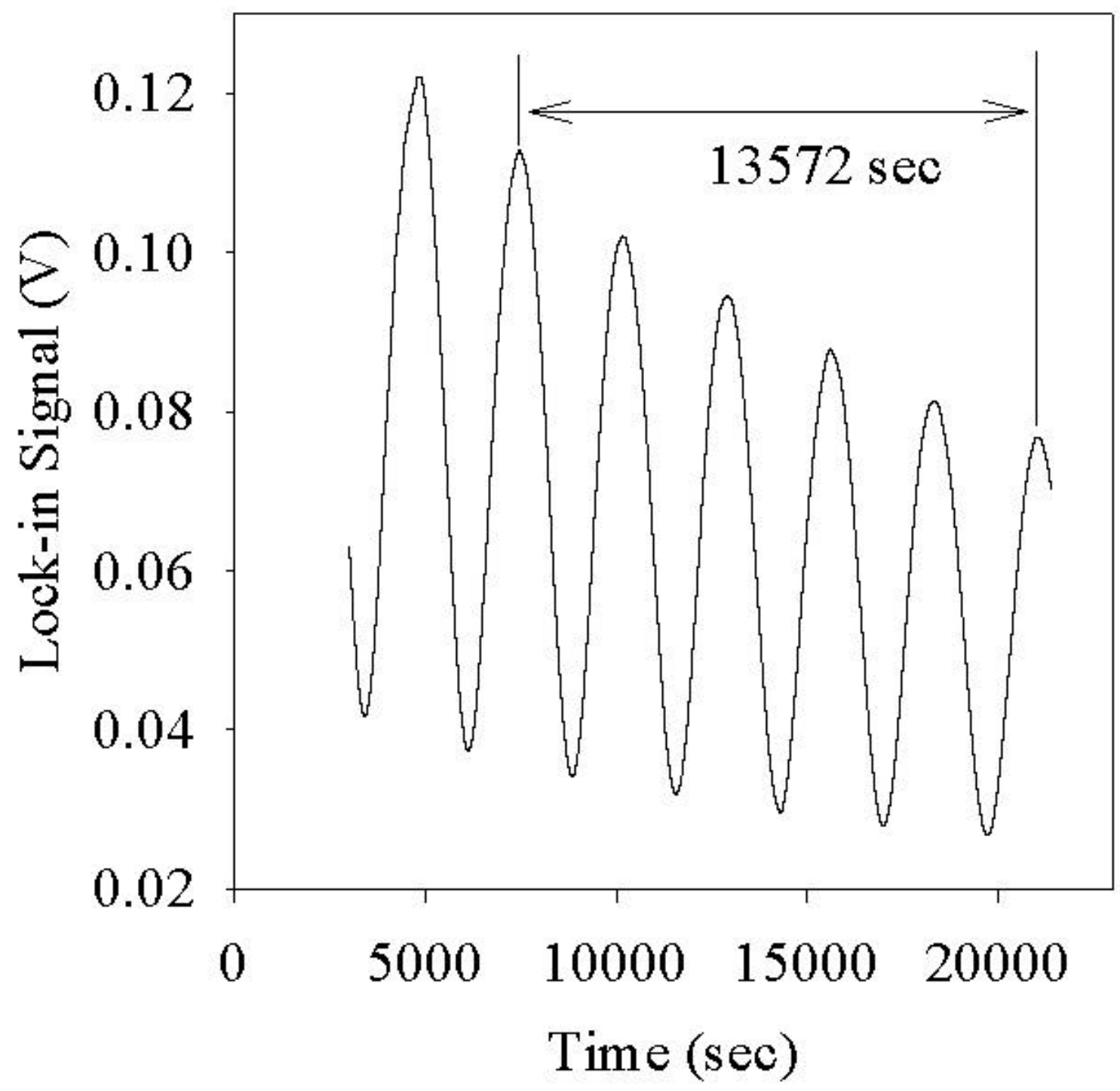

Figure 2. 10 Laser interferometry growth rate measurement of GaN. The time of 13572 seconds over 5 fringes equates to a growth rate of $\sim 0.18 \mu \mathrm{m} / \mathrm{hr}$ in our system. 
constant is then used to determine a sample's growth rate in situ by measuring the period of interference fringes.

\subsubsection{Desorption Mass Spectrometry}

In order to determine the processes involved in the growth of $\mathrm{GaN}$, in situ characterization has to involve the ability to "see" the surface chemistry taking place. One way to do this is through DMS. DMS can be used to study the growth kinetics of semiconductors, and can provide valuable insights into the growth processes. For example, DMS has been used in the GaAs system to monitor and control desorption fluxes allowing for the accurate composition determination of AlGaAs layers grown by MBE. $^{25}$ In GaN, DMS has shown that Ga atoms bond to the surface in two different configurations, each with a distinct energy, ${ }^{26}$ and has been used to accurately determine in situ growth rates. ${ }^{27}$ It has been proposed that hydrogen plays a significant role in the growth kinetics of GaN. ${ }^{26}$ DMS can be used to investigate this effect, as shown in more detail in Section 3.4. DMS uses a mass spectrometer oriented normal to the sample surface. Using apertures to restrict the field of view of the mass spectrometer and "image" only the sample surface, it is possible to measure what species are desorbed or reflected. When combined with measurements of the incoming flux of material, one can determine the relative amount of different species being incorporated into the growing film.

The mass spectrometer used in the current experiments is a UTI 100C 1-400 atomic mass unit (amu) quadrupole mass spectrometer, which consists of an ionizer, a set of electrostatic lenses, a quadrupole mass filter and an electron multiplier. The ionizer generates electrons that ionize the incoming gas in the ionizer assembly. These ions are then focused into the quadrupole mass filter which, based on the applied DC and AC voltages on the four cylindrical rods, can filter out all ions that do not have the correct charge to mass ratio. Only the ions of interest reach the electron multiplier and are counted as signal. 


\subsection{Ex Situ Characterization}

\subsubsection{Hall Effect}

The Hall effect is an efficient and inexpensive measurement of the electrical properties of semiconductors. The sign and density of the majority carriers can be easily determined, as well as their mobility. In addition, temperature-dependent measurements can extract information about impurity energy levels within the band gap and the concentrations of those impurities. This information is essential for the development of semiconductor devices, and is a very useful research diagnostic. A brief review of the Hall effect and the determination of useful material parameters is included in Appendix B.

A temperature-dependent Hall effect apparatus is used to perform the electrical characterization of samples grown within this laboratory. Samples are mounted on a custom designed sample holder, and fine wires are soldered to the sample with In using the standard Van der Pauw four-point geometry to provide electrical contact. ${ }^{28}$ The sample holder is then placed in a Cryomech GB15 cryostat where the temperature is controlled and monitored by a TRI Research Model T2000 temperature controller using a resistive heating element and diode sensors. The cryostat is evacuated to a pressure less than $10^{-5}$ Torr before cooling using a Balzers TPU330 turbomolecular pumping station. The cryostat is placed between the poles of a Varian water-cooled electro-magnet driven by a Hewlett Packard Model 6456B power supply, with a typical magnetic field of 6 kgauss for these experiments. Measurements are possible between 10 and $400 \mathrm{~K}$, but are often limited to a minimum of $\sim 100 \mathrm{~K}$ due to the contacts becoming non-ohmic.

Current is supplied during measurements to the sample by a Keithley Model 220 current source and monitored by a Keithley Model 485 picoammeter. The various voltages are then monitored using a Keithley Model 196 voltmeter. The current supply, voltmeter, and ammeter are connected using a Keithley 7001 switching unit. This switching unit allows the current to be directed between any combination of electrical contacts, a necessity of the four-probe measurement. The switching unit and temperature controller are connected through an IEEE-488 BUS to a 486 computer for systemization 
of measurements and calculations of resistivity, Hall coefficient, Hall mobility, and carrier concentration. All electrical leads external to the cryostat are Keithley triax cable.

\subsubsection{Photoluminescence Spectroscopy}

Exciting a semiconductor with above band gap light produces excess electrons and holes. These excess carriers will find any number of pathways to reach their initial, unexcited state. Many of these recombination pathways will be radiative in nature, meaning that they will emit light with an energy specific to that transition. Very useful information can be gained about defects and impurities in the crystal that have formed energy levels in the band gap by analyzing the spectral nature of the emitted light, or luminescence.

Photoluminescence (PL) measurements were performed in Prof. Nancy C. Giles' laboratory within the WVU Department of Physics as part of the collaborative efforts on compound semiconductors. PL spectra were generated at $4.5 \mathrm{~K}$ using a $\mathrm{He}-\mathrm{Cd}$ laser at $325 \mathrm{~nm}$ as the excitation source. The PL was dispersed by a $0.64 \mathrm{~m}$ monochromator and detected by a GaAs photomultiplier tube working in photon counting mode. All spectra were corrected for the measurement system response, and energies were corrected for strain by using low temperature reflectance measurements to identify free excitonic transitions. The low temperature reflectance measurements were also performed in the same lab.

\subsubsection{Raman Spectroscopy}

Raman scattering is the interaction of light with the optical phonon modes of oscillation in a crystal. In this process, part of the energy that is provided by a photon incident on the sample surface gives rise to a phonon, while the rest of the energy is emitted as light with a slightly smaller energy. This emitted light can be detected and analyzed to determine properties of the crystal lattice. Of particular importance in this study is the $E_{2}$ transverse optical phonon mode of wurtzite GaN. The frequency of this mode is not dependent on the carrier concentration of the sample and can thus be used as 
a determination of the strain due to impurity incorporation. ${ }^{29,30}$ A typical Raman measurement of GaN is shown in Figure 2.11.

Room temperature Raman spectroscopy was performed in Prof. Nancy C. Giles' laboratory in the WVU Department of Physics. Spectra were recorded with a Jobin-Yvon Ramanor U-1000 double monochromator with a microprobe attachment. A GaAs-cathode photomultiplier tube (Hamamatsu R943-02) and photon-counting electronics were used to detect the Raman signals. The room temperature emissions were excited by the $476 \mathrm{~nm}$ output of an Innova 400-10 argon-ion laser from Coherent, Inc. An Olympus BH-2 microscope focused the beam to a spot size of about 20 microns in diameter on the sample surface.

\subsubsection{Secondary Ion Mass Spectrometry}

Secondary ion mass spectrometry (SIMS) measurements were carried out on a number of the samples in this study. All SIMS measurements were performed at Evans Analytical Group, Charles Evans and Associates (Sunnyvale, Ca). A high-energy beam of ions, typically oxygen or cesium, is used to sputter a crater into a sample during the SIMS measurement. The ejected material is analyzed by mass spectrometry to determine what chemical species composed the bulk of the material. In this way, a depth profile is obtained. For instance, a plot can be generated for the concentration of boron in GaN vs the depth in the sample. This type of information is extremely useful when attempting to look at the incorporation and diffusion of both intentional and unintentional impurities in a semiconductor. Appendix $\mathrm{C}$ contains a table of the elements measured as well as their uncertainties and minimum detectable levels.

\subsubsection{Auger Electron Spectroscopy}

Auger electron spectroscopy (AES) is another surface sensitive technique for the analysis of the chemical make-up of materials. Here, a high-energy beam of electrons is used to excite atoms on the surface of the material. These high-energy electrons will knock a core electron out of its orbital, creating a core-level vacancy where another electron can fall. When another electron from that atom falls into the vacated core spot, 


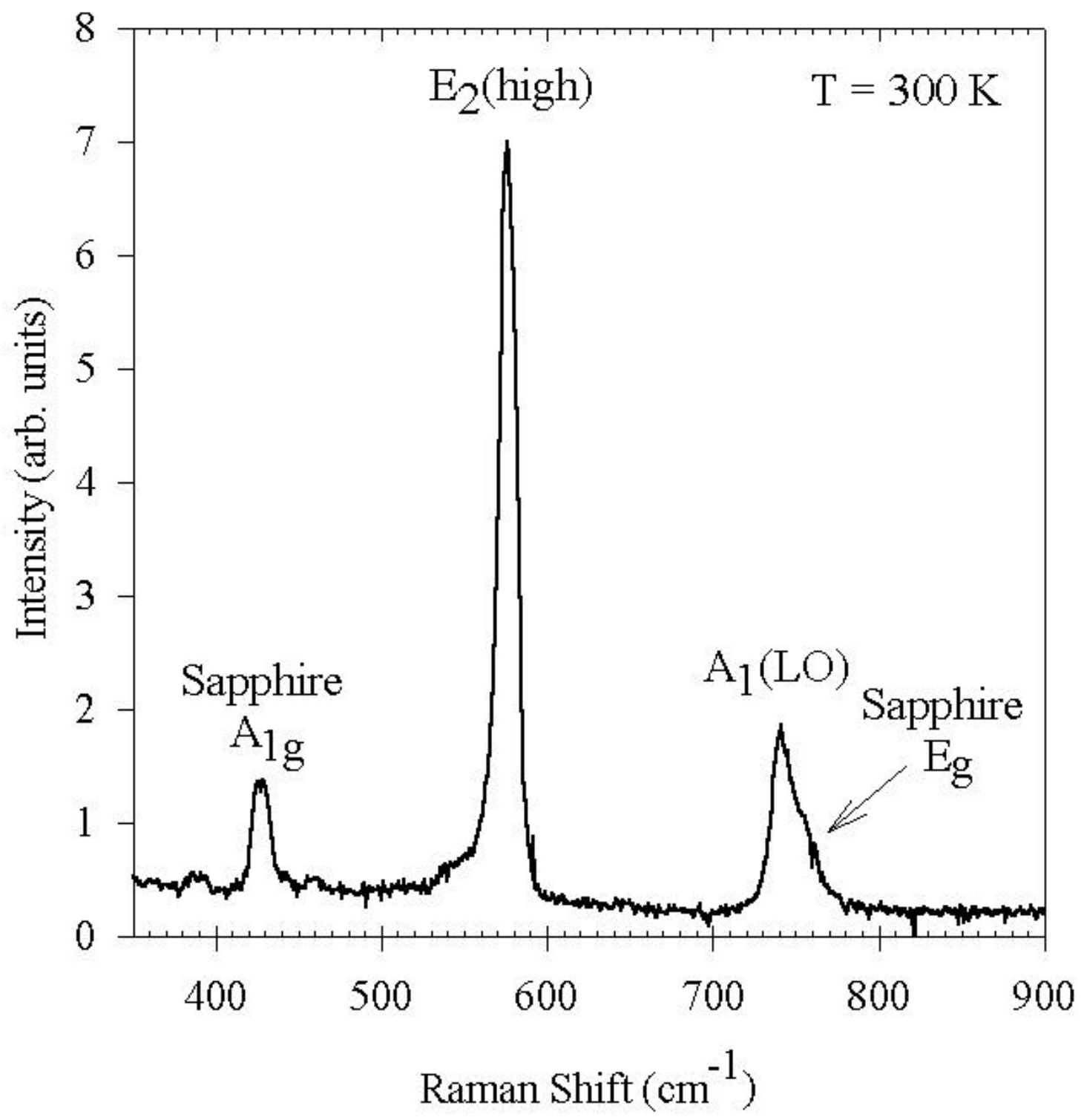

Figure 2.11 A typical Raman measurement of $\mathrm{GaN}$. The position of the $\mathrm{E}_{2}$ peak is used to measure the strain in the sample. 
one pathway for the released energy is to excite an outer electron (the Auger electron) which is ejected from the atom. The Auger electron has a kinetic energy characteristic of the energy levels of atom from which it was ejected. The mean free path for Auger electrons in most materials is on the order of several angstroms, but if the ejected Auger electron is close enough to the surface of the material, its energy can be analyzed and its parent atom can be identified. By scanning through a range of kinetic energies, the atomic make-up of the surface can be found. This technique is useful for many purposes, including study of the nitridation of sapphire (Chapter 3) and in the study of surface segregation effects (Chapters 4 and 5).

AES measurements were performed in Prof. Charter D. Stinespring's lab in the Department of Chemical Engineering at WVU. The system includes a Phi 545 Scanning AES Microprobe with Model 110A Cylindrical Electron Optics. Scans were performed with a $3 \mathrm{keV}$ incident electron beam, a beam current of $2 \mu \mathrm{A}$ and a nominal spot size of $3 \mu \mathrm{m}$. 


\section{Chapter 3: Nitrogen Source Issues and Growth Kinetics}

\subsection{Basic GaN Growth Issues}

\subsubsection{Polarity of the Wurtzite GaN Crystal}

The wurtzite phase of $\mathrm{GaN}$ is the most thermodynamically stable configuration at standard conditions, although the zinc-blende phase can be achieved by epitaxial growth upon a substrate with cubic symmetry. The wurtzite phase belongs to the space group $C_{6 v}^{4}$ and consists of two interpenetrating hexagonal close packed lattices, one Ga lattice and one $\mathrm{N}$ lattice, separated by $5 / 8$ of the distance along the c-axis. Four atoms of the opposite type form tetrahedral bonds with each $\mathrm{Ga}$ atom and each $\mathrm{N}$ atom. A ball and stick model of the structure is shown in Figure 3.1.

One feature of the wurtzite structure is that the two opposite directions along the c-axis are crystallographically inequivalent. That is, there exists no set of symmetry operations that will map the $+\mathrm{c}$-axis direction onto the $-\mathrm{c}$-axis direction. By convention, the $+\mathrm{c}$-axis, or the (0001) direction, is taken to be a vector starting with a Ga atom on the origin pointing to a second Ga atom directly above. The vector denoting the (0001) direction is shown in Figure 3.1. The termination along this direction is referred to as the Ga-face, or Ga-polar surface. The opposite direction, the $(000 \overline{1})$ direction, is known as the $\mathrm{N}$-face or N-polar surface.

The structure of the crystal and the relatively ionic bonds induce a built-in spontaneous electric polarization. In addition to this equilibrium polarization, stress induced, or piezoelectric polarization can also be present. Some advantageous consequences of this polarization allow modulation doping to produce a high mobility two-dimensional electron gas (2DEG) at an $\mathrm{AlGaN} / \mathrm{GaN}$ heterostructure interface, and the formation of piezoelectronically induced sheet carriers. ${ }^{31,32}$ Electron sheet densities of $5 \times 10^{13} \mathrm{~cm}^{-2}$ have been predicted for this interface. ${ }^{31,33}$ 


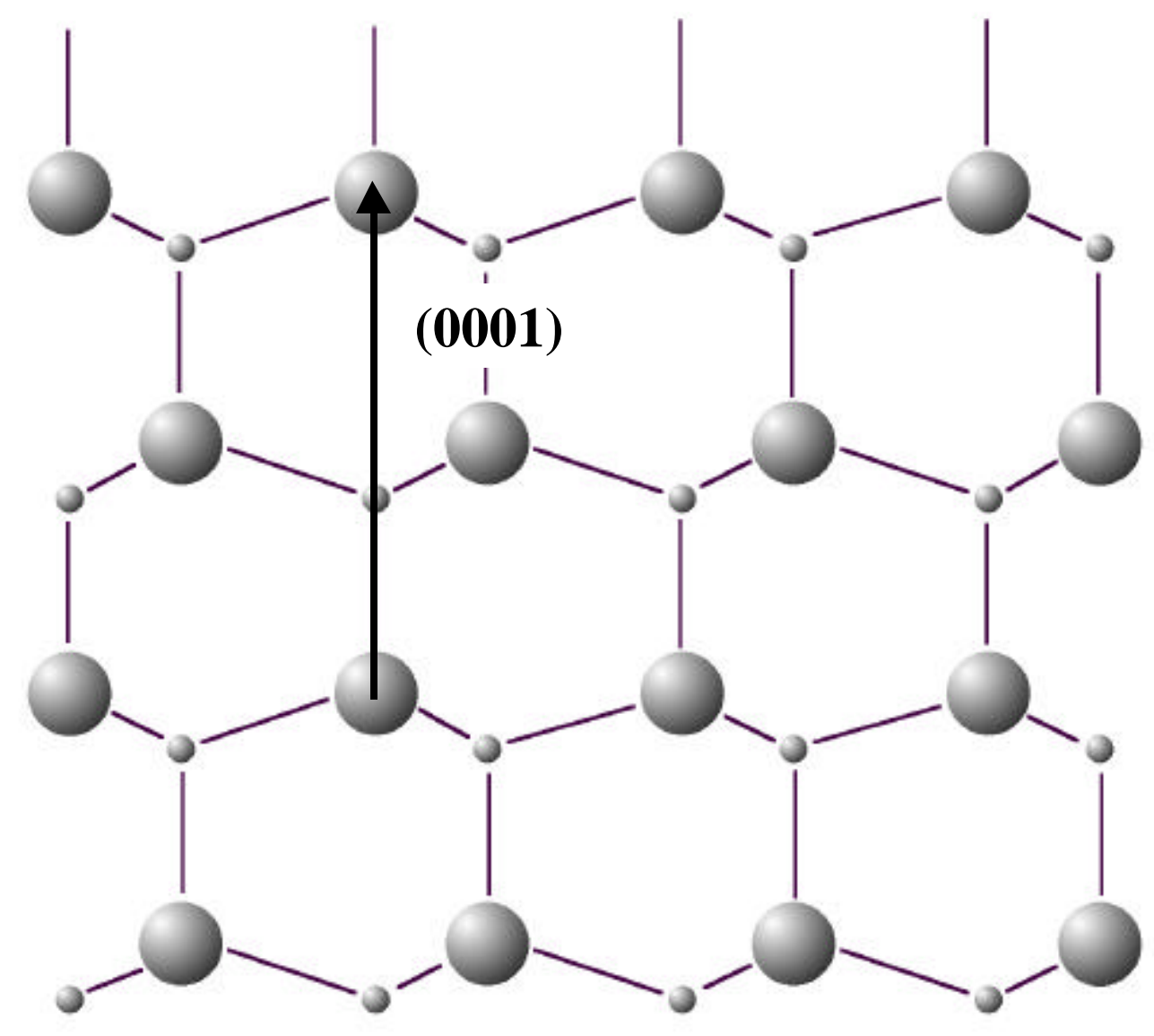

[0001]

\section{Gallium}

Nitrogen

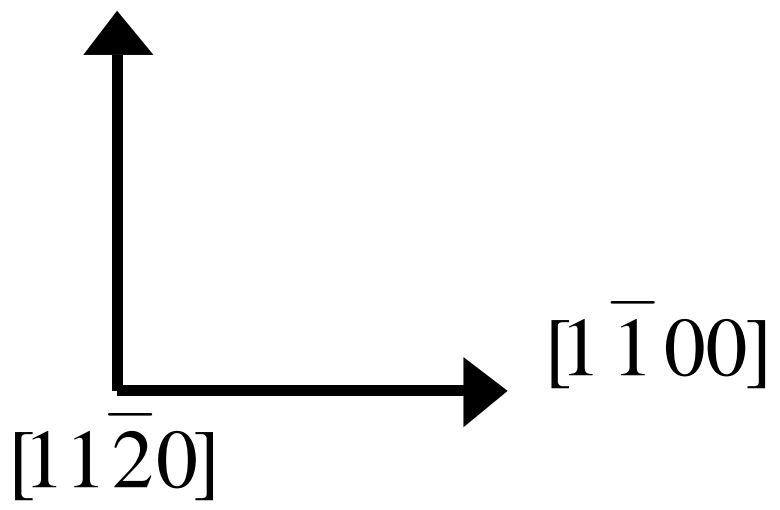

Figure 3.1 Ball and stick model of wurtzite GaN. The convention used for the determination of the (0001) direction is indicated, as well as the two other principal symmetry directions. 
Crystal polarity in this system is also important during growth because of the different chemical properties belonging to each surface. It is known from work in other compound semiconductor systems, such as GaAs, that each polar direction displays different chemical properties. For example, the Ga-terminated (111)A face of GaAs tends to grow more slowly and smoothly than the As-terminated (111)B face. The Ga-polar surface of $\mathrm{GaN}$ is preferred for most applications because it promotes growth with a smooth surface and displays greater chemical stability. The N-polar surface, on the other hand, shows less chemical resistance and often has a surface morphology during MOCVD growth consisting of hexagonal pyramidal hillocks. N-polar GaN also readily decomposes in a hydrogen atmosphere at elevated temperatures.

Growth by MOCVD has been optimized to produce Ga-polar material although films with both polarities can be grown. Techniques for growing Ga-polar material by

MBE have only been developed recently. ${ }^{34,35}$ Growth of GaN by MBE utilizing a 'standard' low temperature buffer layer on sapphire substrates (including all growths in the WVU MBE Lab) almost always grow as N-polar material. It is difficult to perform experiments on both polarities of $\mathrm{GaN}$ using $\mathrm{MBE}$, as in this study, when it is only possible to nucleate one polarity. It is therefore necessary to obtain Ga-polar GaN from an outside source in the form of MOCVD- or HVPE-grown GaN wafers. Fortunately, there seem to be no problems with the regrowth of MBE GaN on these wafers. TEM studies have shown that the MBE/MOCVD interface is virtually invisible. TEM measurements have been made on a number of the samples presented in this work, and no structural trace of the MBE/MOCVD interface has yet been found.

\subsubsection{Ga-Stable vs. N-Stable Growth}

An abrupt transition in the growth morphology of GaN occurs as the conditions are changed from Ga-stable (an excess of $\mathrm{Ga}$ on the growth surface) to N-stable (an excess of $\mathrm{N}$ on the growth surface). This is typically seen as a transition in the RHEED pattern from a streaky pattern indicative of two-dimensional growth to a spotty, transmission-type pattern representative of rough, three-dimensional growth as the III/V ratio is decreased. Representative streaky and spotty RHEED patterns are shown in 
Figure 3.2. We have measured the $\mathrm{Ga} / \mathrm{N}$ ratio of this transition to be unity, in agreement with another study, ${ }^{36}$ both by determining where growth rates are $\mathrm{N}$-limited or Galimited, and by using DMS to determine when the desorbed Ga flux becomes zero. A plot of the growth rate of GaN vs. Ga flux is shown in Figure 3.3 for constant $\mathrm{N}$ source parameters. In this case, below a Ga BEP of $\sim 4-5 \times 10^{-7}$ Torr the growth rate begins to decrease, indicating that the growth is now limited by the amount of $\mathrm{Ga}$ available, i.e. $\mathrm{N}$-stable growth. The "knee" on this graph where the growth rate begins to decrease is where the streaky-to-spotty RHEED transition occurs. As the Ga flux is lowered from Ga-stable conditions, the RHEED pattern brightens. This brightening of the RHEED pattern is associated with the disappearance of the adsorbed layer (most likely at least two adlayers) of Ga from the surface. ${ }^{37}$ This surface is not stable as several minutes of GaN growth at these conditions will turn the diffraction pattern spotty. Numerous AFM and STM studies of samples grown both Ga- and N-stable support the change in growth morphology. ${ }^{19,23,24}$ Ga-stable GaN growth results in a smooth, microscopically flat morphology, while $\mathrm{N}$-stable samples appear rough, as one would expect from the RHEED patterns associated with each regime. The spotty nature of the RHEED pattern and the rough surface morphology are believed to arise from kinetic roughening due to limited Ga diffusion on a N-rich surface. ${ }^{23,38}$ This transition in morphology occurs for both polarities of $\mathrm{GaN}$, but has a somewhat different dependence on the substrate temperature. As can be see in Figure 3.4, for higher substrate temperatures the Gapolarity requires a Ga flux higher than that on the N-polar surface in order to keep the RHEED pattern streaky. This indicates that any excess Ga atoms on the Ga-polar surface are less tightly bound than on the N-polar surface. This also indicates that the N-polar surface is more reactive and that Ga desorption plays more of a role for growth on the Ga-polar surface.

\subsection{Determination of Active Nitrogen Species}

The chemical composition of the nitrogen species generated in an rf-plasma source is typically a complex mixture of molecules, atoms, ions and molecular metastables. Neutral molecular nitrogen in its ground state is inert at the growth 


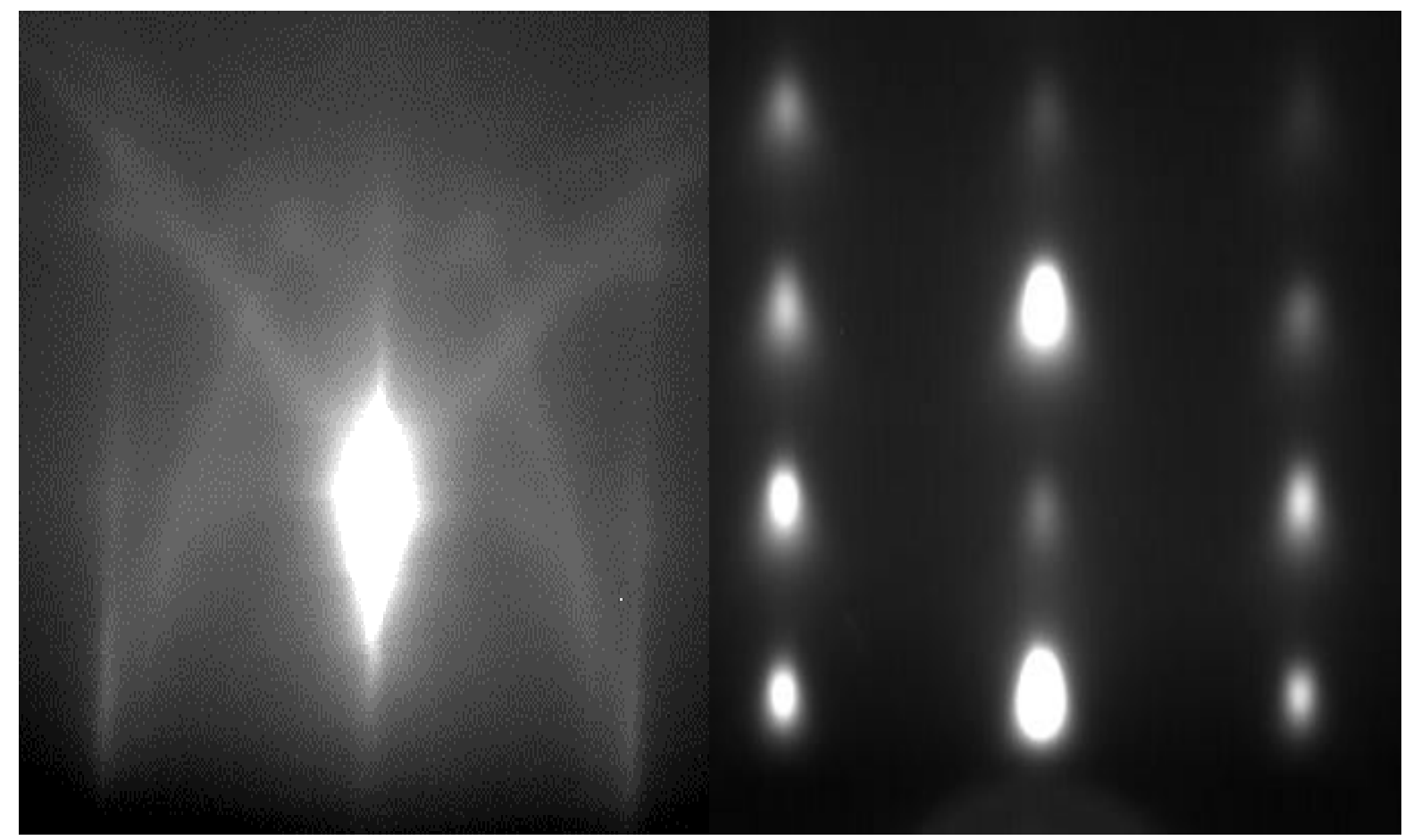

Figure 3.2 Typical RHEED images of streaky (left) and spotty (right) patterns. 


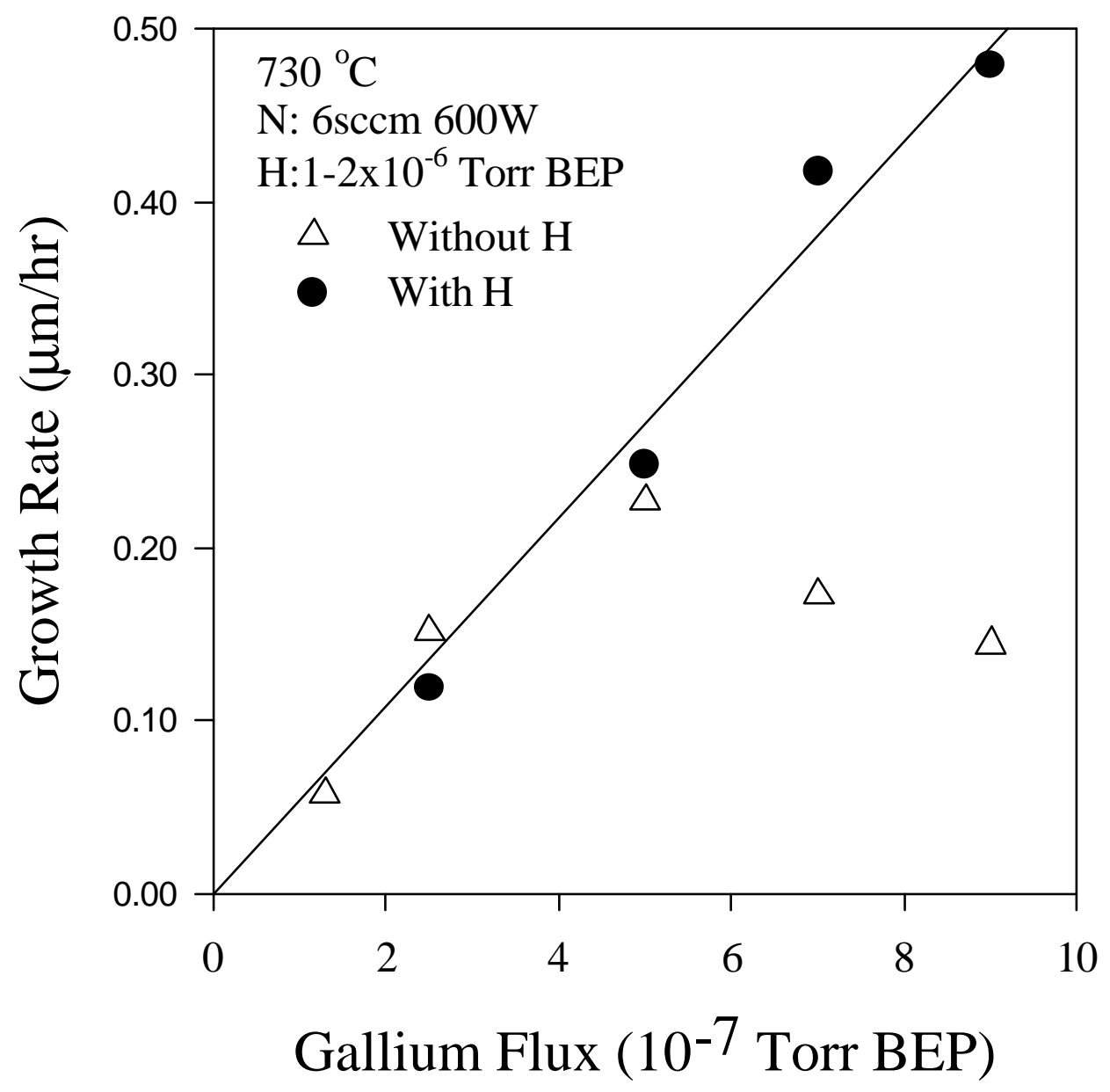

Figure 3.3 GaN Growth rate vs. Ga flux. The "knee" on the plot for growth without atomic hydrogen represents the transition from a three-dimensional to a two-dimensional growth mode. 


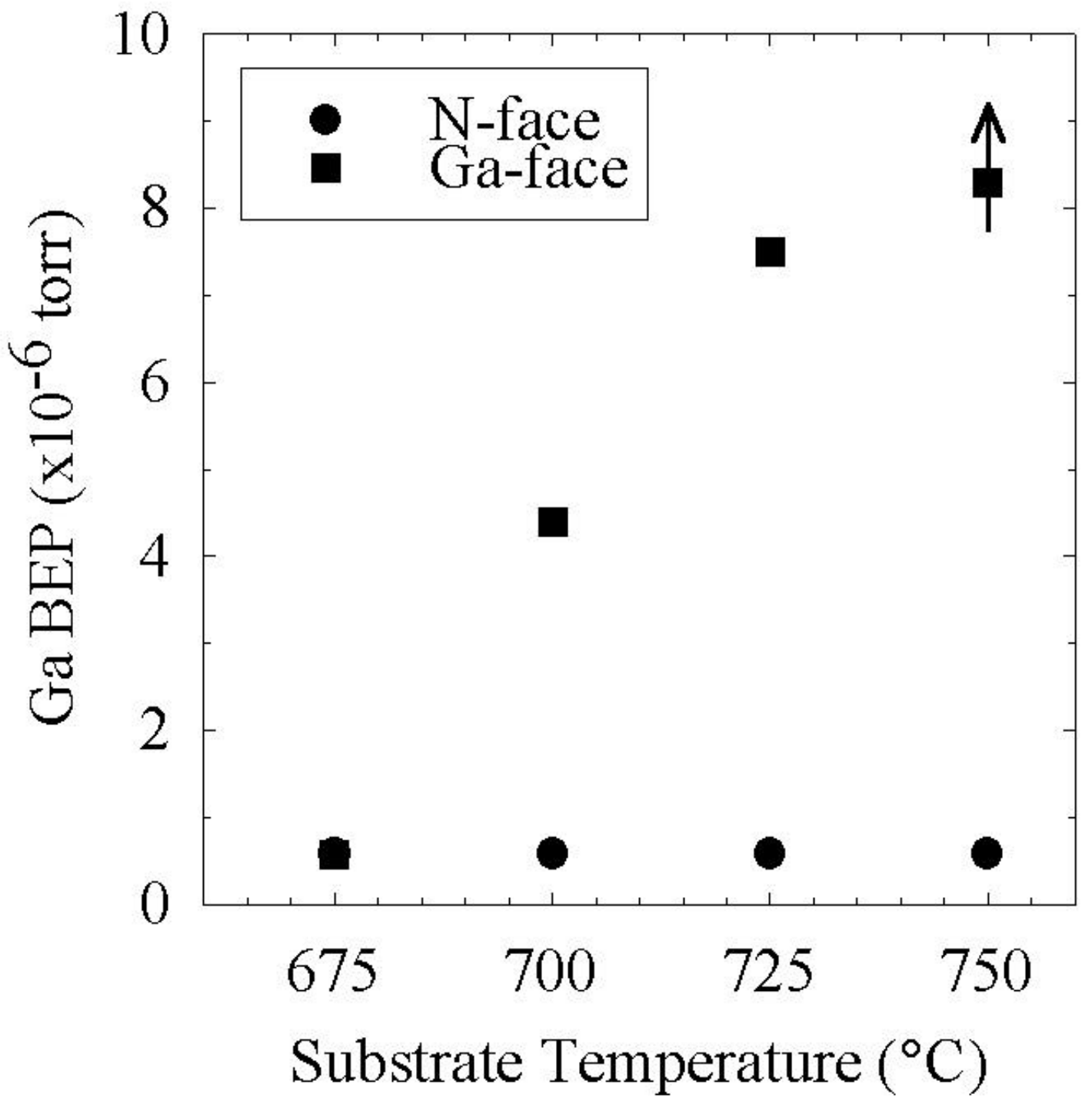

Figure 3.4 Ga flux necessary to keep the RHEED pattern streaky for both polarities of $\mathrm{GaN}$. Note that the point at $750^{\circ} \mathrm{C}$ for the Ga polarity is only a lower limit as a higher flux could not be reached with the current source. 
temperatures used in MBE. For the growth of nitride-based materials it is necessary, and indeed of paramount importance to determine the constituent ingredients that are generated by the plasma source for the purpose of understanding the growth kinetics and mechanics. The quality of semiconductors in general, and $\mathrm{GaN}$ in particular, is greatly influenced by the type of active species used as will be shown in this chapter.

The analysis of the species of active nitrogen emitted from an rf-plasma source can be complicated. It is worth noting that it is not necessarily what is generated in the plasma itself that matters, but rather what species of nitrogen reach the growth surface. Most spectroscopic studies of active nitrogen have been performed by examining the light generated inside the plasma cell. The EPI Unibulb rf-plasma source (described below) allows this measurement as there is a direct line-of-sight into the plasma cell through an optical port at the back of the source. The matter is somewhat more complicated in the Oxford CARS-25 source (also described below), as the light must first travel through a pyrolytic boron nitride $(\mathrm{PBN})$ cell to be collected. Some of the light generated in the plasma is blocked or attenuated making analysis of the experimental data more difficult. Again, it is important to note that this light is representative of processes inside the plasma cell, and not necessarily what is reaching the growth surface. By analyzing this light, it is impossible to account for any interaction of the active species with the walls or aperture of the plasma cell, or gas-phase interactions once the plasma has left the cell. A much less ambiguous way of determining the active flux is to use a mass spectrometer in direct line-of-sight of the plasma source as was done in this study. This allows for the direct determination of what nitrogen species are being emitted from the source.

\subsubsection{Description of the Energy Levels of Nitrogen}

Figure 3.5 shows the energy levels of the nitrogen molecule. Of particular interest for this work are the chemically inert ground state, the first ionized state with a potential energy of $\sim 15.5 \mathrm{eV}$, and the metastable $A^{3} \Sigma_{u}^{+}$state with an energy approximately $6 \mathrm{eV}$ above the ground state. This metastable state has a relaxation time of 


\section{Activated Nitrogen GaN Reaction}

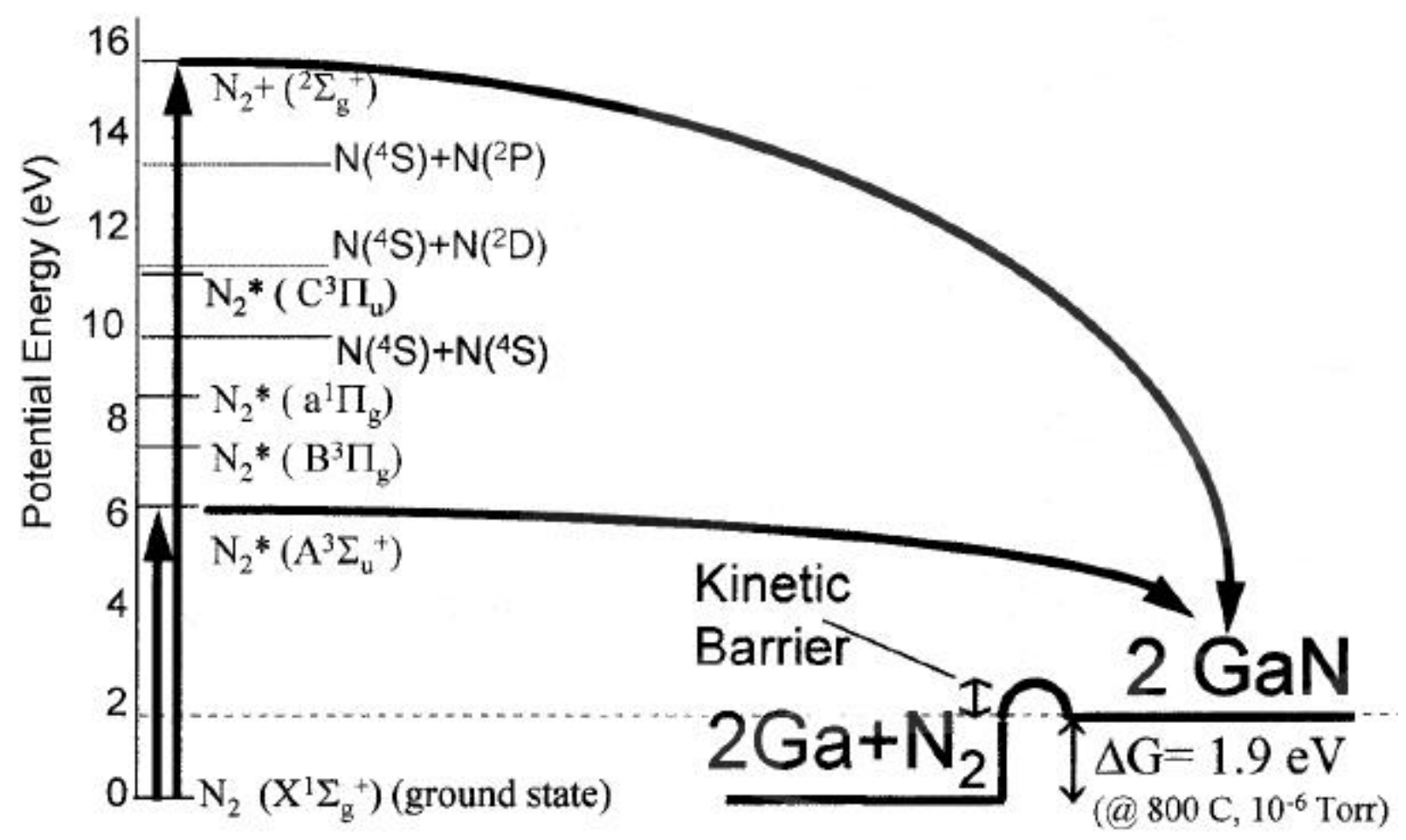

Figure 3.5 Energy levels of molecular nitrogen and the formation energy of GaN. (Courtesy N. Newman, Northwestern University) 
1.3 \pm 0.3 seconds, ${ }^{39}$ considerably longer than normal decay times of nano- to microseconds. The thermal energy can be used to determine the minimum velocity of the nitrogen molecules. Given an estimated plasma cell temperature of $720^{\circ} \mathrm{C},{ }^{40}$ a nitrogen molecule would take less than $1 / 3 \mu \mathrm{sec}$ to travel the $24.2-\mathrm{cm}$ distance from the nitrogen source to the substrate. This time is significantly shorter than the lifetime of the metastable molecule.

\subsubsection{Description of the rf-Plasma Sources and Experimental Set-up}

The nitrogen species from two rf-plasma sources were investigated. The first is a CARS-25 source from Oxford Applied Research (Oxon, UK). A schematic of this source is shown in Figure 3.6. This source has a removable aperture plate allowing the investigation of various aperture configurations while maintaining the same overall conductance. The configuration of the aperture plate was found to have a profound influence on the type of active nitrogen emitted from the source. Four apertures were investigated for the Oxford source, ranging from one large hole to 255 smaller holes, as shown in Table 3.1.

The second source was the EPI Unibulb rf-plasma source from Applied EPI, Inc. (St. Paul, MN). After the initial characterization of the Oxford source, it became clear that more and smaller holes in the aperture plate were preferable to larger ones. With this in mind, discussions commenced with Applied EPI about the fabrication of an aperture with a large number of small exit holes. What resulted is now the standard Unibulb cell, which features 400 holes each with a diameter of $0.2 \mu \mathrm{m}$. This configuration results in an approximate $50 \%$ increase in conductance over the standard Oxford source configuration.

The characterization of these sources was performed in the laboratory of Prof. Charter D. Stinespring in the Department of Chemical Engineering at WVU. The current work involving the $\mathrm{N}$ source characterization was built upon the research of several previous students at WVU. Work directed toward the Master of Science degree was performed by S.L. Buczkowski ${ }^{41}$ in the Department of Physics and S. Kumar ${ }^{42}$ in the 


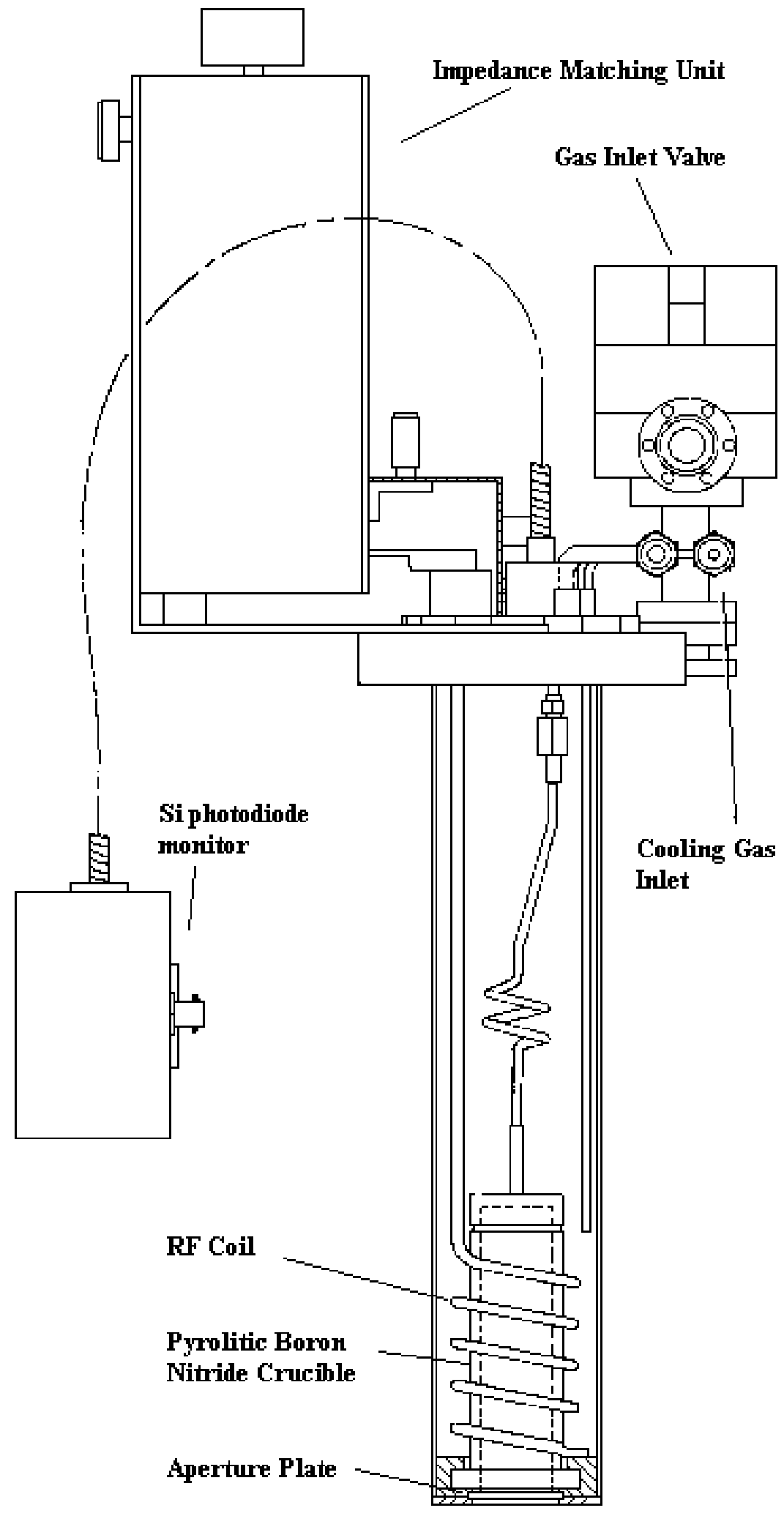

Figure 3.6 Schematic drawing of the Oxford CARS-25 rf-plasma nitrogen source. 
Table 3.1. Typical flux of ions and atomic nitrogen from the rf-plasma sources, and the actual incorporation rate into GaN.

\begin{tabular}{|c|c|c|c|c|c|c|}
\hline \multirow[b]{2}{*}{ SOURCE } & \multicolumn{2}{|c|}{ APERTURE } & \multirow[b]{2}{*}{$\begin{array}{l}\text { Ion Flux } \\
\left(10^{13} \text { ions }\right. \\
\left.\mathrm{cm}^{-2} \mathrm{~s}^{-1}\right)\end{array}$} & \multirow{2}{*}{$\begin{array}{c}\text { Max. } \\
\text { Ion } \\
\text { Energy } \\
(\mathrm{eV})\end{array}$} & \multirow[b]{2}{*}{$\begin{array}{l}\text { N Atom Flux } \\
\left(10^{15} \text { atoms }\right. \\
\left.\mathrm{cm}^{-2} \mathrm{~s}^{-1}\right)\end{array}$} & \multirow[b]{2}{*}{$\begin{array}{l}\mathrm{N} \text { incorporation } \\
\text { in } \mathrm{GaN}\left(10^{15}\right. \\
\left.\text { atoms } \mathrm{cm}^{-2} \mathrm{~s}^{-1}\right)\end{array}$} \\
\hline & $\begin{array}{c}\text { Hole } \\
\text { Diameter } \\
(\mathrm{mm})\end{array}$ & $\begin{array}{c}\text { Number } \\
\text { of } \\
\text { Holes }\end{array}$ & & & & \\
\hline \multirow{4}{*}{$\begin{array}{c}\text { OXFORD } \\
\text { CARS-25 } \\
600 \mathrm{~W} \\
6 \mathrm{sccm}\end{array}$} & 3.0 & 1 & a) & $>65$ & a) & a) \\
\hline & 1.0 & 9 & 7.6 & $40-50$ & 4.5 & 0.58 \\
\hline & 0.5 & 37 & 3.8 & $15-25$ & 3.0 & 0.26 \\
\hline & 0.2 & 255 & 2.3 & $8-12$ & 2.3 & 0.19 \\
\hline $\begin{array}{l}\text { EPI Unibulb } \\
600 \mathrm{~W}, 2 \mathrm{sccm}\end{array}$ & 0.2 & 400 & .003 & $<3$ & 0.63 & 1.9 \\
\hline $\begin{array}{l}\text { EPI Unibulb } \\
300 \mathrm{~W}, 2 \mathrm{sccm}\end{array}$ & 0.2 & 400 & .001 & $<3$ & 0.28 & 1.1 \\
\hline
\end{tabular}

a) not measurable 
Department of Chemical Engineering on the initial mass spectrometry and optical characterizations of the Oxford source. M.R. Millecchia ${ }^{20}$ extended the work on the Oxford source and performed the initial studies on the EPI source. This work also led to the Master of Science degree in the Department of Physics.

The sources were mounted in a UHV chamber in direct line-of-sight to an Extrel quadrupole mass spectrometer whose repeller grid was biased separately to determine ion energies. In addition, a stainless steel electrostatic plate located below the plasma source could be used to completely deflect ions out of the flux. This plate allows for the quantification of neutral species in the plasma. The ionizer energy was set high enough to ionize both atomic and molecular nitrogen (typically $>15 \mathrm{eV}$ ), but below the dissociation threshold of molecular nitrogen $(<28 \mathrm{eV})$. The experimental set-up is shown in Figure 3.7. The 28-amu (molecular nitrogen) and 14-amu (atomic nitrogen) peaks were monitored for various operating conditions of both the source and the mass spectrometer, while the total nitrogen flux was calculated from the pressure and the known geometry and pumping speed of the analysis chamber. In this way, the active nitrogen species were determined for the EPI source as well as the four different configurations of the Oxford source.

\subsubsection{Results}

As mentioned previously, determining the effect of various active nitrogen species on layer growth is complicated when using rf-plasma sources. These sources typically produce a complex mixture of active nitrogen superimposed on a background of inert molecular nitrogen, as illustrated by Table 3.1. The operating conditions shown are for those resulting in a maximum growth rate $(600 \mathrm{~W}$ at a nitrogen gas flow rate of $6 \mathrm{sccm}$ for the Oxford source, $600 \mathrm{~W}$ at a nitrogen gas flow rate of $2 \mathrm{sccm}$ for the EPI source), plus one intermediate condition $(300 \mathrm{~W})$ for the EPI source. The ion flux given is for the total ion flux, that is atomic plus molecular ions. The atomic ion flux was typically two to three times larger than that of the molecular ions, with both distributions peaked near the maximum energies. The maximum ion-energy range listed in Table 3.1 is representative of the difference between molecular and atomic ionic species. Use of 


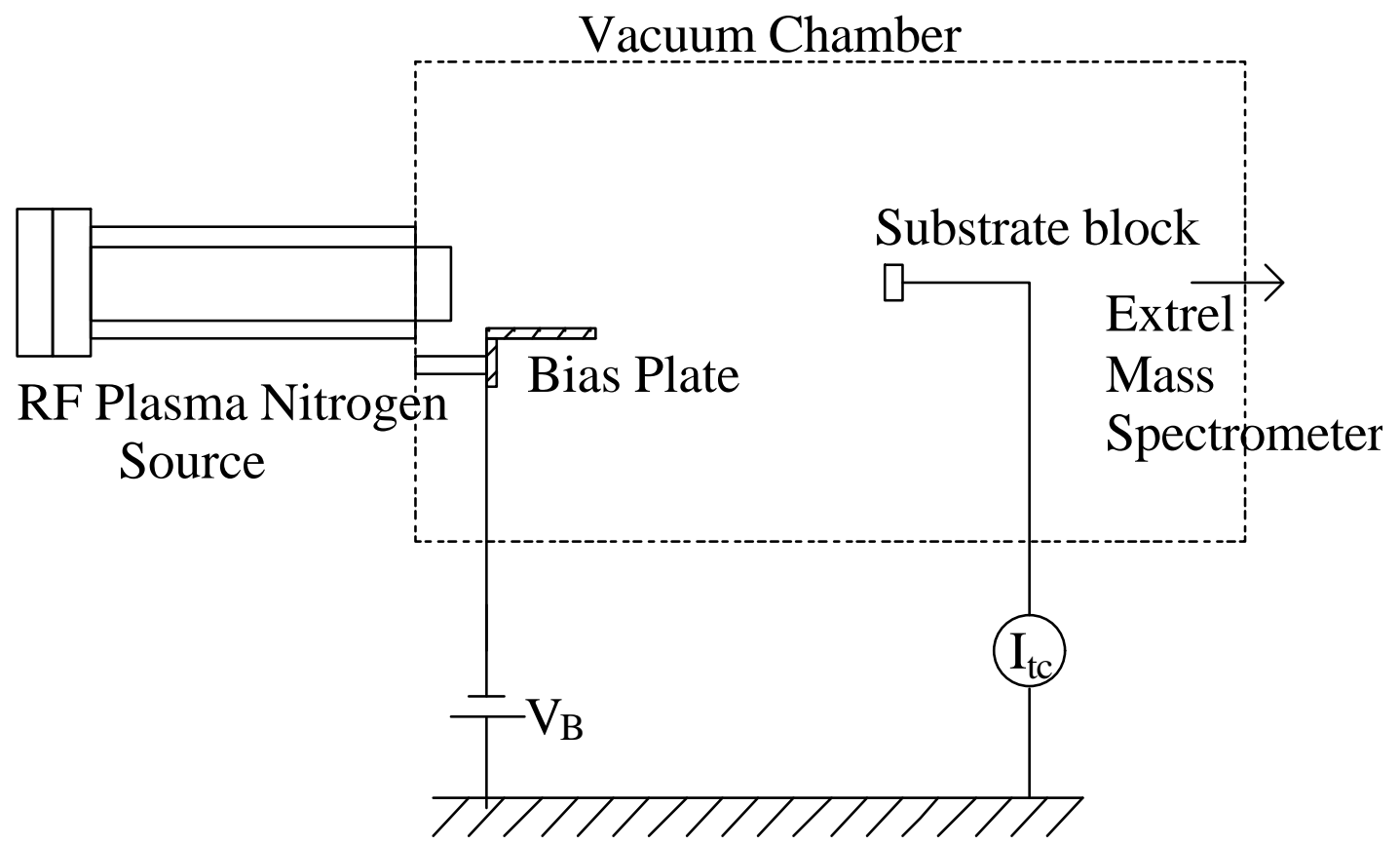

Figure 3.7 Schematic drawing of the experimental set-up used for the characterization of the rf-plasma sources. 
the four different exit hole configurations on the Oxford source led to a varying mixture of atomic nitrogen and ions. Similar results have been reported for apertures used on an ECR source. $^{43}$ Atomic nitrogen accounted for $2-6 \%$ of the total nitrogen flux, with the ion content reduced to less than $0.1 \%$ as the number of holes increased (and the size of the holes decreased). The maximum ion energy for the Oxford source ranged from greater than $65 \mathrm{eV}$ for the single hole aperture to about $10 \mathrm{eV}$ for the 255 -hole aperture. Another source parameter, the incorporation efficiency, was determined by measuring the nitrogen actually incorporated in $\mathrm{GaN}$ based on the growth rates from separate $\mathrm{MBE}$ growth experiments compared to the total nitrogen impinging on the growing layer. Measured growth rates were too large to result from the ions and so must be due to the atomic nitrogen. While the incorporation efficiency scaled well with atomic nitrogen concentrations, it is clear that a significant fraction of the atomic nitrogen is not incorporated into the growing layer. Approximately one in ten $\mathrm{N}$ atoms contribute to growth.

Several significant differences were observed upon characterization of the EPI source. First, the total ion content was significantly reduced, to less than $3 \times 10^{-7}$ of the total flux, with the remaining ions having energies less that $3 \mathrm{eV}$. Second, the incorporation efficiency was significantly larger, with the surprising result that the measured atomic nitrogen flux was far too low to account for the increase in growth rate. During the EPI source characterization, we found considerable quantities of molecular nitrogen ions could be produced with the ionizer energy set approximately $6 \mathrm{eV}$ lower than normally necessary to ionize molecular nitrogen. As seen in Section 3.2.1, this energy difference corresponds to the $A^{3} \Sigma_{u}^{+}$metastable state of the nitrogen molecule, and indicates this source produces a significant amount of molecular nitrogen metastables. The observation of metastables in the EPI source flux is consistent with a previous spectroscopic study indicating that excited molecular nitrogen is generated in the plasma inside the source. ${ }^{44}$ The presence of the metastables complicated the source characterization somewhat, but we were able to determine an atomic nitrogen efficiency of about $2-3 \%$. This source has proven quite effective for the incorporation of nitrogen in $\mathrm{GaN}$ with growth rates at least an order of magnitude too large to be due to the atomic 
nitrogen based on comparison with the Oxford source. Since the ion content is negligible, the most likely active nitrogen species remaining is the molecular nitrogen metastable.

It can be seen from Table 3.1 that neither source produces enough ionic nitrogen to account for the observed growth rates, indicating that growth is due to other nitrogen species. The Oxford source configurations studied produced primarily atomic nitrogen, with little indication of the presence of molecular metastables during characterization. Atomic nitrogen appears to be relatively inefficient for growing $\mathrm{GaN}$, requiring about ten atoms in the flux for each one incorporated into the growing layer. In contrast, the EPI source configuration used produced significantly less atomic nitrogen and yet gave a factor of three-to-five increase in growth rate indicating that metastable molecular nitrogen is the dominant active nitrogen specie in this source. This is an interesting result since analysis of the optical spectrum generated inside the EPI plasma cell indicates the presence of a large concentration of atomic nitrogen. ${ }^{45}$ It is known from this study that decreasing the size of the holes in the aperture dramatically decreases the concentration and energy of nitrogen ions. It is conceivable that interactions of nitrogen atoms with the aperture holes helps atoms to recombine into molecules and perhaps promotes the formation of molecular nitrogen metastables. This would explain the difference in the concentrations of different active species present inside and outside the plasma cell.

\subsection{Determination of the Relative Reactivity of Various Nitrogen Species by the Nitridation of Sapphire}

Now that the components that comprise the nitrogen flux emanating from each source configuration had been determined, and more importantly control of the dominant active specie was achieved, it was necessary to determine the relative reactivity of each specie. This was accomplished by determining the time necessary in order for each type of active nitrogen to nitride sapphire. 
The nitridation of sapphire is a common step in the nucleation of $\mathrm{GaN}$ on sapphire. It is used to form a thin layer of what is presumed to be AlN on the sapphire surface by exposure of the sapphire to active nitrogen. The case may be somewhat more complicated than just the exchange of oxygen atoms in the sapphire with nitrogen atoms to form an AlN overlayer. Some authors ${ }^{46,47}$ have found a completely relaxed surface upon nitridation with a final lattice constant equal to that of $\mathrm{AlN}$, while another ${ }^{48}$ found a lattice constant consistently smaller than that of pure AlN. The formation of an $\mathrm{AlO}_{1-\mathrm{x}} \mathrm{N}_{\mathrm{x}}$ alloy has been invoked to explain the smaller lattice constant, but this is unlikely based on thermodynamic considerations, and is contradicted by an in situ transmission electron microscopy study of nitridation. ${ }^{49}$ It is known that the degradation of the PBN crucible in the plasma source acts as a source of boron that can be incorporated into growing layers. We have proposed that the reduced lattice constant is actually due to the initial formation of a $\mathrm{BN}$ layer followed by the growth of an $\mathrm{Al}_{1-\mathrm{x}} \mathrm{B}_{\mathrm{x}} \mathrm{N}$ alloy. ${ }^{50}$ This is supported by the observation of cubic grains in the GaN layer that may be due to the segregation of $\mathrm{BN}$ during nitridation into its cubic phase.

Regardless of the actual process, nitridation may be used to reduce the lattice mismatch between $\mathrm{GaN}$ and sapphire allowing for a greater degree of crystalline perfection during subsequent layer growth. For our purposes, the nitridation rate of sapphire may be used as a measure of the reactivity of a given nitrogen specie.

Nitridation experiments were performed in the same chamber as the rf-plasma source characterization using a heated sample stage. Nitridation at low temperature was chosen to accentuate differences in the relative reactivity of the various species. Nitridation did not occur at room temperature for any source configuration, but proceeded at a reasonable rate at $400^{\circ} \mathrm{C}$, which was used for all the nitridation experiments in this chamber. The nitridation chamber was connected to a separate chamber where Auger measurements could be performed using the apparatus described in Chapter 2. This meant that the samples would not need to be exposed to the air prior to the Auger measurements. 
The analysis of the Auger measurements uses elemental sensitivity factors, ${ }^{51}$ and is representative of the number of atoms of each element present within the Auger sampling depth. Since Yeadon et al. ${ }^{49}$ have shown that $\mathrm{AlN}$ and $\mathrm{Ab}_{2} \mathrm{O}_{3}$ are immiscible and nitridation proceeds by the formation of an $\mathrm{AlN}$ overlayer on the $\mathrm{Al}_{2} \mathrm{O}_{3}$, we used a standard model ${ }^{52}$ for oxygen signal attenuation by an AlN overlayer normalized by the total Al signal to estimate AlN thickness. The model used an experimentally measured oxygen sensitivity factor relative to aluminum from clean $\mathrm{Al}_{2} \mathrm{O}_{3}$ and electron inelastic mean free paths based on the model by Tanuma et al. ${ }^{53}$ The relative shift in Al signal strength between $\mathrm{Al}_{2} \mathrm{O}_{3}$ and $\mathrm{AlN}$ has been taken into account in the model. However, the model did not include backscatter correction factors so the calculated AlN thickness values should be treated as approximate.

Figure 3.8 details the Auger analysis made during a nitridation experiment using the Oxford source with the single-hole aperture. The $\mathrm{N}$ signal begins to increase as the $\mathrm{O}$ signal becomes attenuated, consistent with the formation of an AlN layer on top of the sapphire. Nitridation using each type of active specie measured proceeded in a similar manner, but each had a different associated time scale. Figure 3.9 summarizes AlN overlayer thickness as a function of time for three configurations of the CARS-25 source. Figure 3.9(a) corresponds to the nitridation of two samples using the single-hole aperture, with a large flux $(5 \%)$ of high-energy molecular and atomic nitrogen ions. This condition is similar to an ECR source, and results in a relatively rapid nitridation with a rate of about $0.3 \AA / \mathrm{min}$ for the first $10 \AA$. Figure 3.9(c) is representative of the 9-hole aperture results. There appears to be an initiation stage lasting approximately 180 minutes, followed by AlN formation at a rate of about $0.03-0.04 \AA / \mathrm{min}$. The active flux from this source configuration is a mixture of both atomic nitrogen (5-6\%) and nitrogen ions $(0.1 \%)$. Figure 3.9(b) represents the same configuration, but now the electrostatic deflector plate underneath the path of the nitrogen was used to remove the ions from the flux, as was done during the course of the source characterization. For this atomic nitrogen only configuration, nitridation began immediately, and maintained a rate approximately three times $(0.1 \AA / \mathrm{min})$ that shown in Figure 3.9(c). 


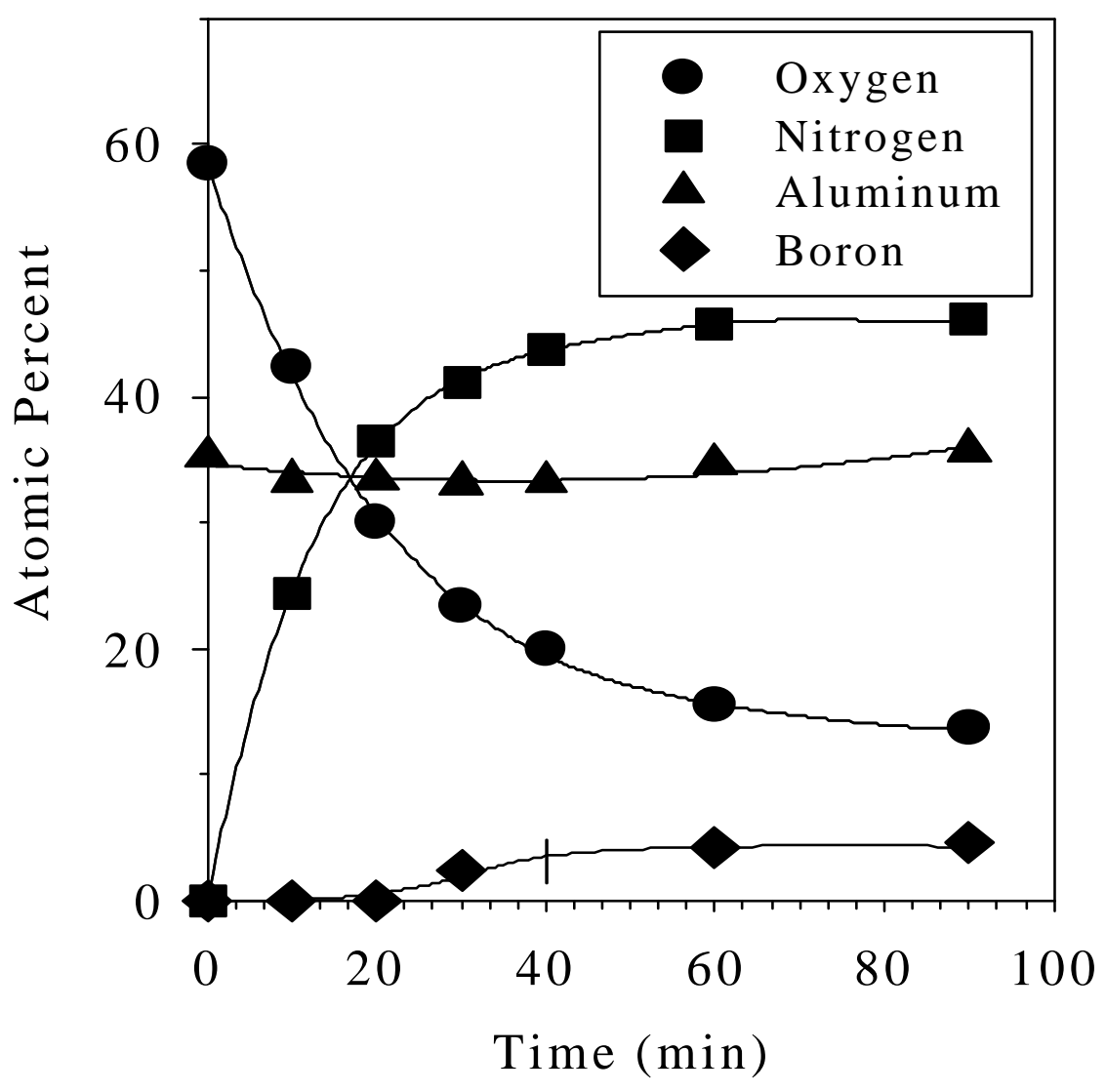

Figure 3.8 Auger analysis of $\mathrm{Al}, \mathrm{O}, \mathrm{N}$, and $\mathrm{B}$ during nitridation of sapphire using the Oxford source with a single hole aperture. The solid lines are guides to the eyes. 


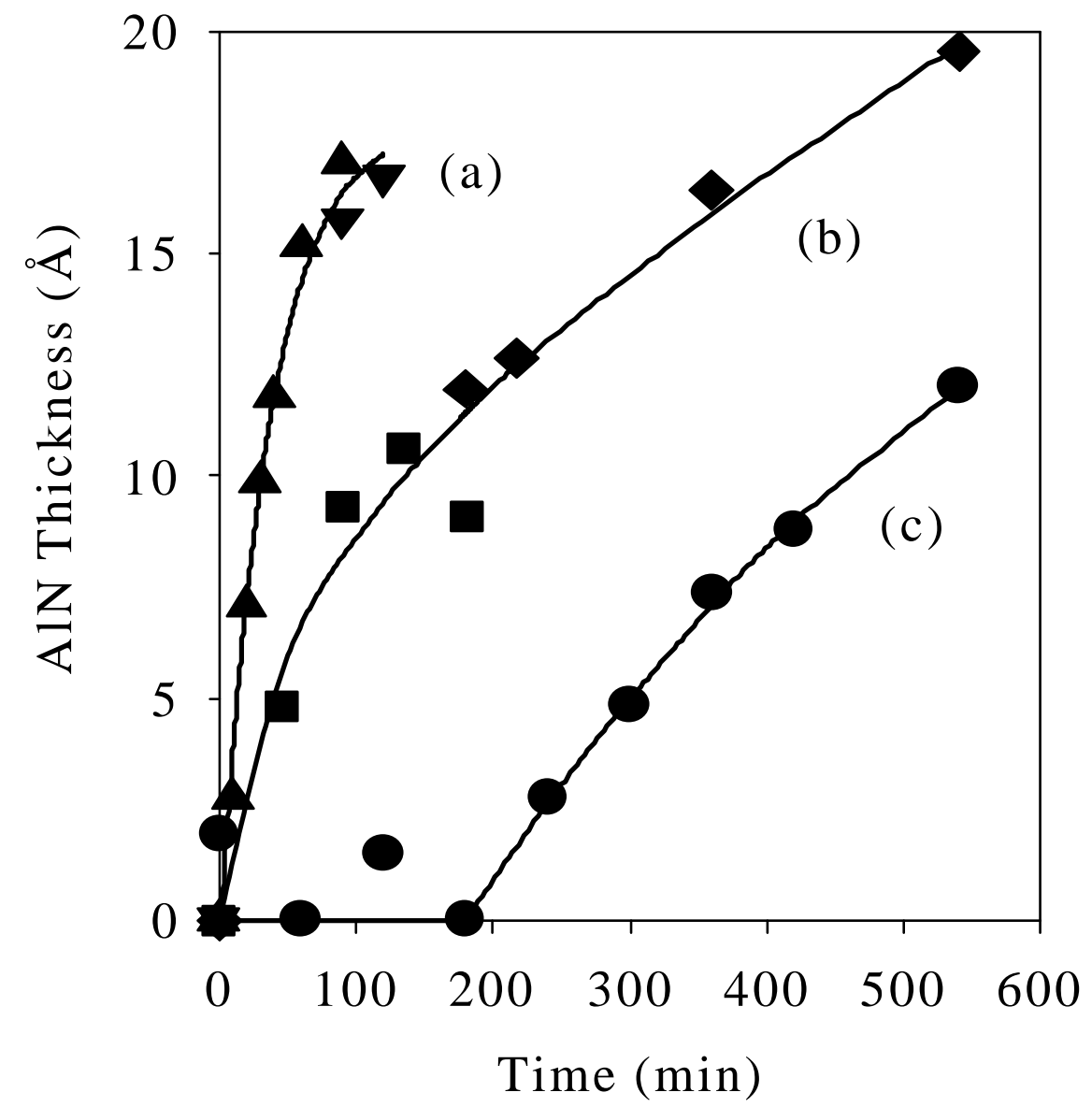

Figure 3.9 AlN overlayer thickness as a function of time for (a) and , single-hole aperture; (b) and , 9-hole aperture with ions deflected; and (c) , 9-hole aperture without ion deflection. The solid lines are guides to the eye. 
The trends shown in Figure 3.9 indicate that both energetic nitrogen ions and atomic nitrogen are quite reactive, with some indication that the energetic ions are the more reactive species. This is particularly evident for larger AIN thickness. Of equal interest is that a flux of lower energy ions apparently suppresses the nitridation process. A likely mechanism is that atomic nitrogen is adsorbed on the growing surface in a weakly bound state before being more tightly bound in the AlN matrix. The weakly bound nitrogen is then attacked by incoming ions (and possibly atoms) forming molecular nitrogen that quickly desorbs, removing these atoms from participating in the nitridation process. The initiation stage represents a period where the adsorbed nitrogen is very weakly bound to the sapphire surface and easily removed. Based on the rapid onset of AlN growth once initiated, it is likely that once AlN is formed, the nitrogen is more strongly bound to the surface and the nitridation process then proceeds at a reasonable rate.

Figure 3.10 summarizes the Auger chemical analysis of nitridation using the EPI source. The results were substantially different from that observed with the Oxford source. Again, the $\mathrm{N}$ signal increases while the $\mathrm{O}$ signal decreases, but now there seems to be a large contribution from surface boron. While boron was observed for nitridation with each Oxford source configuration, the Auger analysis indicates significantly more boron on the $\mathrm{Al}_{2} \mathrm{O}_{3}$ surface exposed to the EPI source. The results imply that an overlayer of $\mathrm{BN}$ was formed within the first 20 minutes of nitridation as indicated by the saturation of the B signal, followed by an apparent cessation of BN formation. During this initial stage, both the $\mathrm{Al}$ and $\mathrm{O}$ Auger signals reduced in proportion, consistent with the formation of a BN overlayer without any contribution from AlN. Surprisingly, both the $\mathrm{B}$ and the $\mathrm{Al}$ signal then became constant, while the $\mathrm{O}$ signal decreased and the $\mathrm{N}$ signal continued to increase. The latter is suggestive that AlN is now growing, although since the $B$ signal also remains constant, it is feasible that an $\mathrm{Al}_{1-\mathrm{x}} \mathrm{B}_{\mathrm{x}} \mathrm{N}$ alloy may actually be forming. The short predicted mean free paths for $\mathrm{B}(7 \AA)$ and $\mathrm{Al}(6 \AA)$ Auger electrons compared to that of $\mathrm{O}(11 \AA)$ or $\mathrm{N}(14 \AA)$ would result in a constant $\mathrm{B}$ and $\mathrm{Al}$ signal with the others still changing. Assuming for comparison that only AlN formation is occurring, 


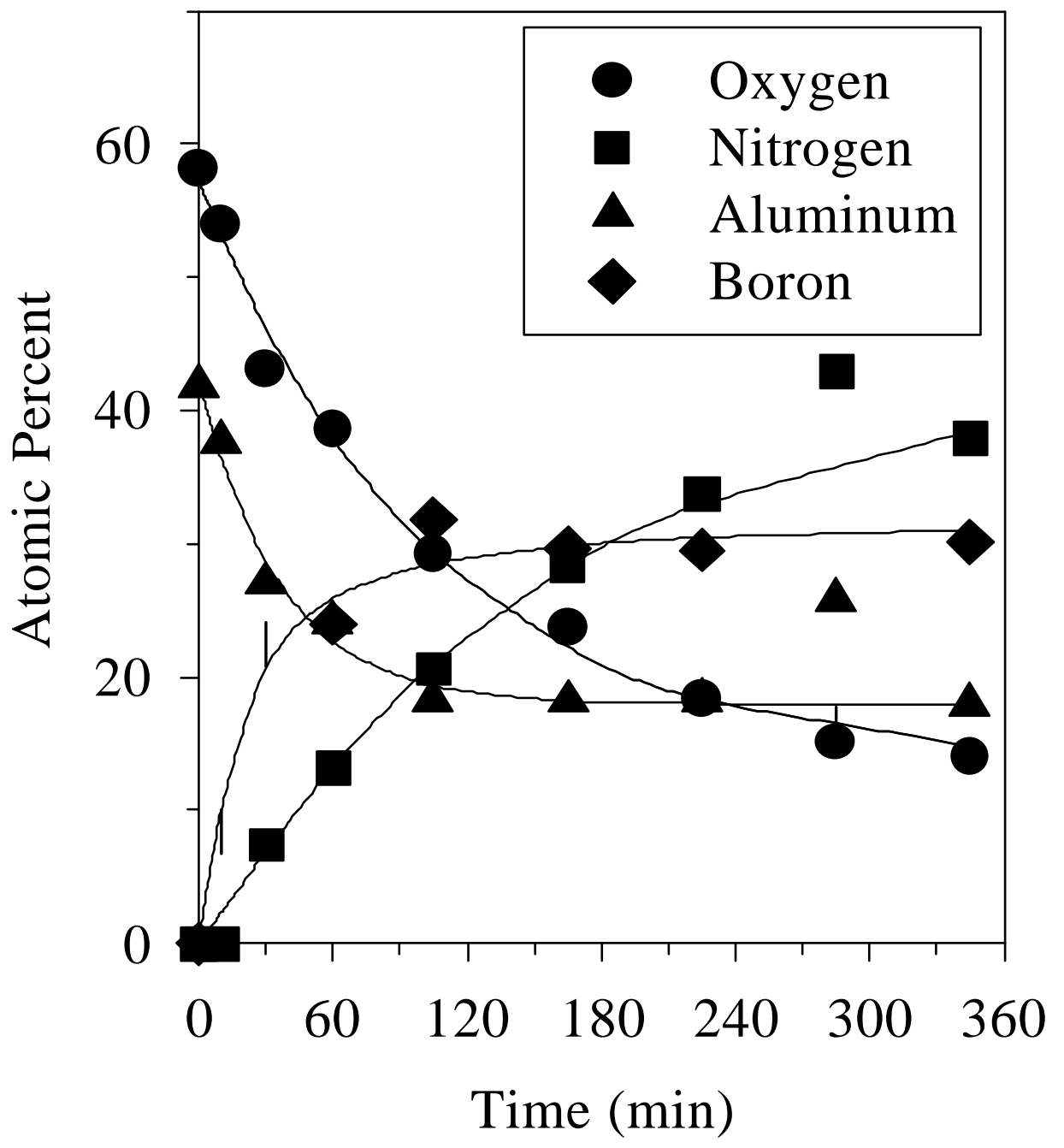

Figure 3.10 Auger analysis of $\mathrm{Al}, \mathrm{O}, \mathrm{N}$, and $\mathrm{B}$ during nitridation using the EPI source. 
modeling interprets an AlN growth rate during this second stage of $0.07 \AA / \mathrm{min}$. This growth rate is consistent with the concentration of atomic nitrogen in the flux coupled with the lack of ions. This latter result may indicate that the relatively large concentration of nitrogen metastables believed to be present is relatively non-reactive, at least as far as nitridation is concerned. Interpretation of this set of Auger measurements, however, is certainly complicated.

The results depicted in Figure 3.10 are suggestive that the flux from the EPI source has a significantly larger B content than the Oxford source, with the source of B from both sources likely due to the degradation of the PBN liner in the source itself. SIMS results from GaN (see Figure 3.11) and doping studies in II-VI materials ${ }^{54}$ indicate the EPI source emits far less B than does the Oxford source, and this result is more indicative of the efficiency of metastable nitrogen for group III-nitride layer growth. Figure 3.11 summarizes the B content for two GaN layers measured using SIMS. Figure 3.11(a) represents the B concentration in a layer grown using the Oxford source with the 9-hole aperture, yielding an average B concentration of about $8 \times 10^{19} \mathrm{~cm}^{-3}$. Similar results, although not shown, were obtained for the other aperture sets, and also after replacing the PBN liner in the Oxford source. The EPI source actually emitted significantly less $\mathrm{B}$, with incorporation levels in the mid- $10^{17}$ to $1 \times 10^{18} \mathrm{~cm}^{-3}$ range, as shown by Figure 3.11(b). The variation in B concentration for each trace corresponds to different source rf-powers, with the minimums corresponding to $200 \mathrm{~W}$ and the maximums to $600 \mathrm{~W}$. A similar difference in $\mathrm{B}$ incorporation has also been reported to occur with these sources for nitrogen doping of $\mathrm{ZnSe}$ and CdTe. ${ }^{54}$

Consequently, the rapid formation of a BN overlayer by the EPI source coupled with the smaller concentration of actual $\mathrm{B}$ emitted from this source suggest that the $A^{3} \Sigma_{u}^{+}$ nitrogen metastables are a particularly efficient specie for the growth of III-N compounds. This is further supported, as shown in the next section, by the significant increase in nitrogen incorporation efficiency (and hence growth rate) observed for MBE growth of GaN with the EPI rf-plasma source. 


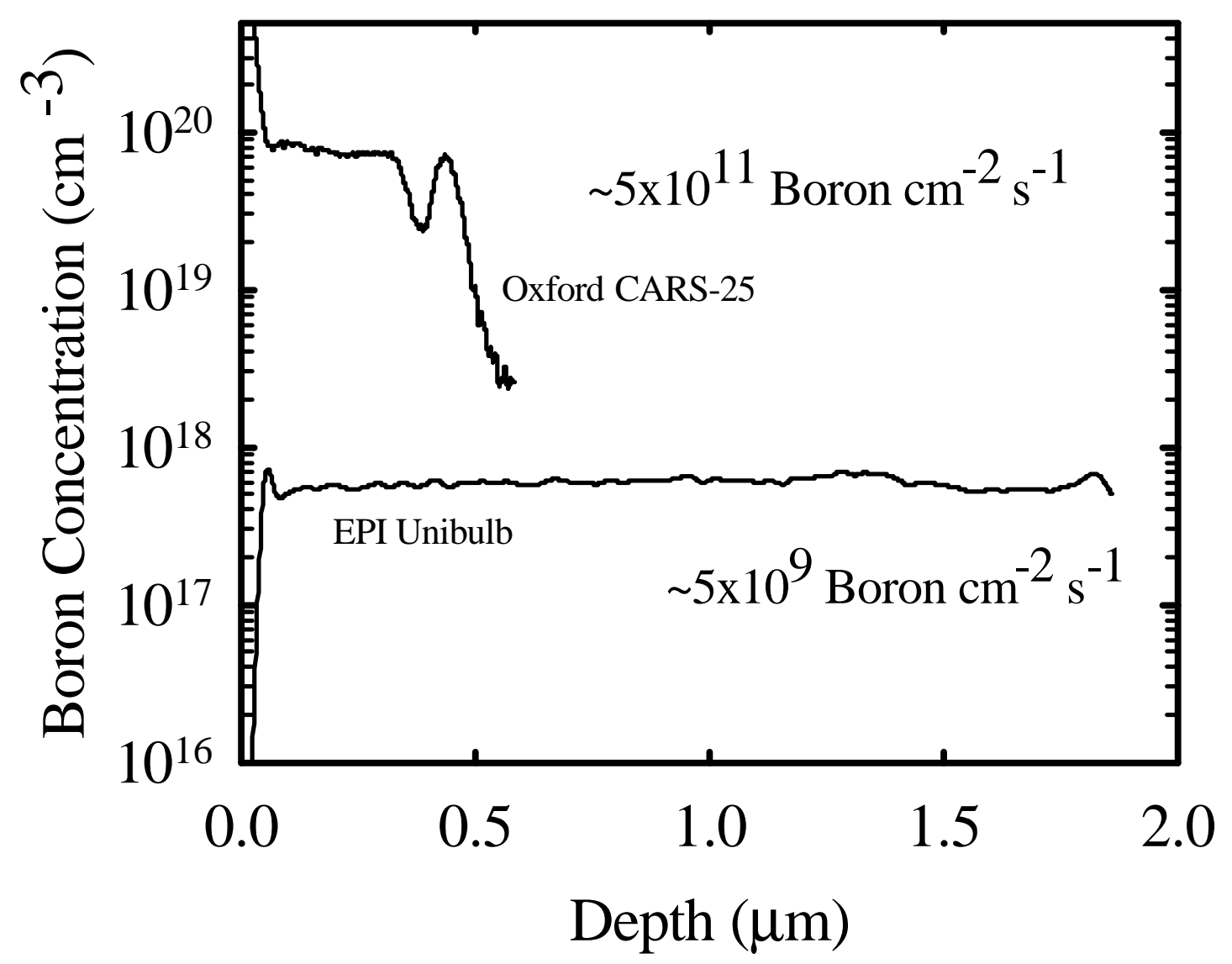

Figure 3.11 SIMS measurement of the boron concentration in GaN samples grown by two different rf-plasma sources. 
RHEED measurements of the lattice constant variation during nitridation of sapphire were performed using our EPI source. The RHEED pattern was monitored continuously for sapphire exposure to the active nitrogen flux for about 180 minutes at temperatures of 200,400 and $700^{\circ} \mathrm{C}$. In contrast to previous results, ${ }^{48}$ no evidence was observed for any nitridation at $200^{\circ} \mathrm{C}$. The differences in the fraction of active species found in different rf-plasma sources can possibly explain this effect. ${ }^{55}$ Evidence of nitridation was readily observed at 400 and $700^{\circ} \mathrm{C}$. Immediately after active nitrogen exposure, diffraction streaks began to appear that are generally attributed to the replacement of oxygen by nitrogen in the sapphire lattice. An additional 10 to 15 minutes of exposure to active nitrogen resulted in the disappearance of the underlying sapphire pattern.

Figure 3.12 illustrates the evolution of the lattice parameter of the nitridation layer measured using RHEED. The measurements were calibrated using the bulk lattice parameters of sapphire. The variation in lattice constant appeared to reach an initial plateau, followed by a second increase to a final value that remained stable for longer times. In general, this final measured lattice constant was several percent lower than the $3.11 \AA$ lattice constant of AlN, in agreement with Widmann et al. ${ }^{48}$ The lattice constant tended to be significantly smaller for the higher temperature nitridation. Faint and very diffuse rings appear superimposed over the streaks in the RHEED pattern at about the time of the second increase in lattice constant.

It is obvious that B can play a significant role during the long periods required for nitridation using an rf-plasma source. Auger analysis indicates a final layer that contains about 10 to $20 \%$ BN. This is consistent with RHEED lattice constant measurements that also imply significant amounts of BN. If this were due to alloy formation, the Auger scans shown in Figures 3.9 and 3.11 would imply approximate x-values of 0.04 and 0.4 respectively. The RHEED studies indicated similar compositions assuming that $\mathrm{Al}_{1-\mathrm{x}} \mathrm{B}_{\mathrm{x}} \mathrm{N}$ is being formed. RHEED analysis consistently indicates a lattice constant closer to that 


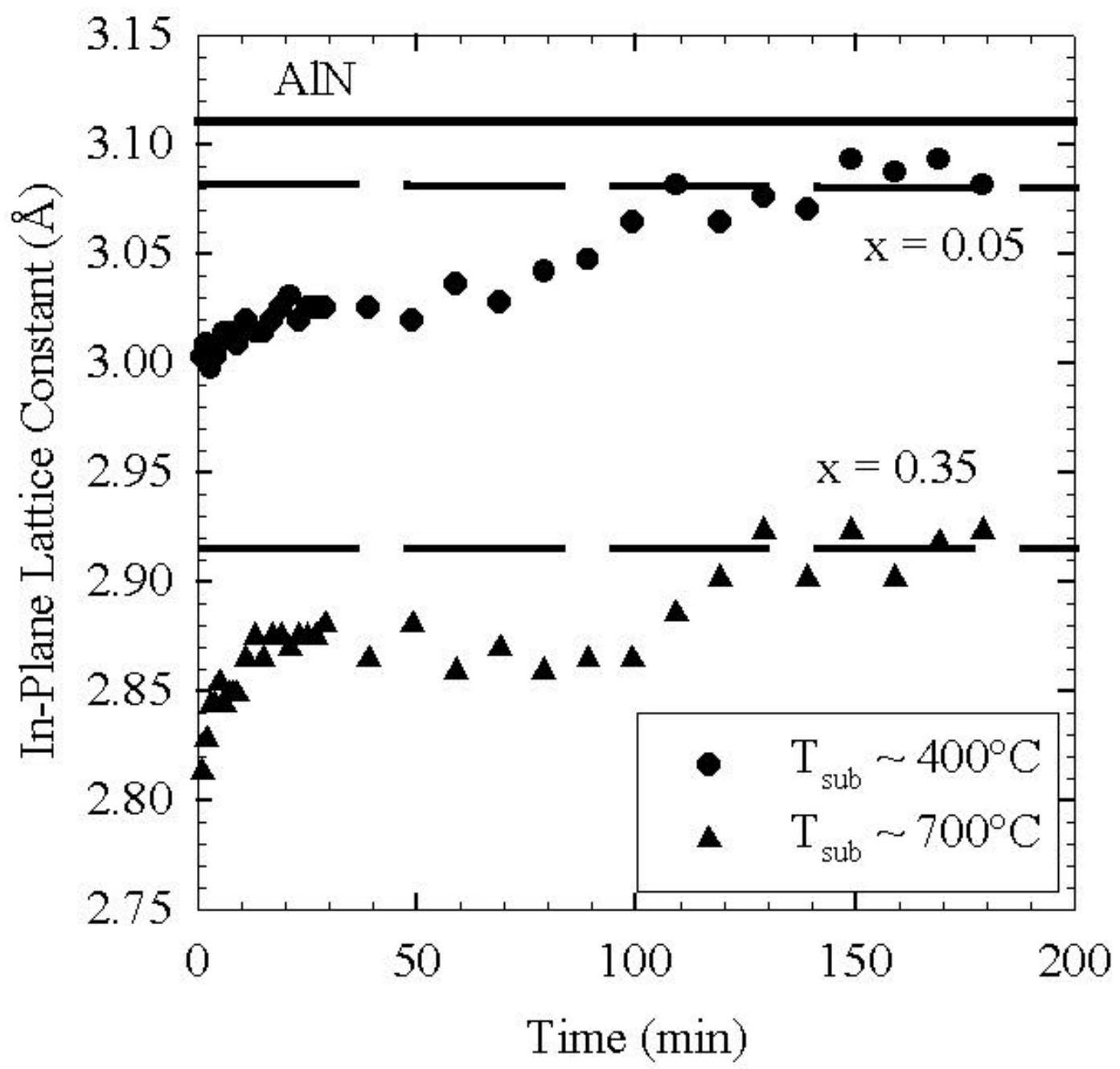

Figure 3.12 Variation of the in-plane lattice constant with nitridation time as observed using RHEED. The $x$-values represent the composition of $\mathrm{Al}_{1-\mathrm{x}} \mathrm{B}_{\mathrm{x}} \mathrm{N}$ that would result in this lattice constant. 
of pure AlN for lower temperature nitridation implying that the higher temperatures are more effective for B capture in the evolving nitridation layer.

Polyakov et al. ${ }^{56}$ studied the growth of AlBN solid solutions by MOCVD. They found that for compositions larger than about $\mathrm{x}=0.01$ phase segregation occurred into $\mathrm{BN}$ and low $x$-value AlBN. The implication is that high $x$-value AlBN is thermodynamically unstable. In Polyakov's study, the second B-rich phase appeared to be wurtzite BN. These measurements cannot identify if this is occurring in these nitridation layers. It is possible that high $\mathrm{x}$-value AlBN can be formed for the thin nitridation layers. However, it is also quite likely that phase segregation is indeed occurring, particularly for the more B-rich layers found at higher temperatures. The onset of segregation may be indicated by the relatively abrupt second rise in lattice constant observed by RHEED, accompanied by the appearance of faint, diffuse rings. Indeed, the presence of both the faint ring pattern and the strong, streaky pattern is indicative of an inhomogeneous surface. Widmann et al. found that lower nitridation temperatures led to reduced nucleation of cubic grains in subsequent AlN buffer layer growth. Based on the current nitridation results, a possible mechanism explaining this effect is the formation of larger $\mathrm{x}$-value AlBN at higher temperatures, followed by segregation and nucleation of cubic $\mathrm{BN}$ inclusions once a critical thickness is reached. Polyakov et al.'s study suggests that the majority of the B-rich phase may maintain the wurtzite structure, with higher temperatures and larger B concentrations necessary to form the cubic phase.

\subsection{Effect of Various Nitrogen Species on the Growth of GaN}

While the nitridation experiments give an indication as to the reactivity of the different active nitrogen species, their effect on III-N layer formation may be quite different. It is clear that nitrogen ions and atoms are highly reactive. In fact, they may be too reactive for the successful growth of $\mathrm{GaN}$ as highly reactive species would tend to promote decomposition of the surface while less reactive species would not. This certainly seems to be the case with the molecular nitrogen metastables, which appear to be relatively non-reactive during nitridation, but particularly effective during the growth of GaN and, unintentionally, BN. Now that each source has been characterized and the 
predominant active specie has been found, it is possible to determine the effect of various active species on the growth of GaN.

\subsubsection{Growth Rate Measurements}

Figure 3.13 illustrates the relative growth rate of $\mathrm{GaN}$ as a function of temperature for various configurations of the EPI and Oxford sources. Growth using a predominantly atomic nitrogen flux (the Oxford source) always led to the early onset of decreased growth rate with increasing substrate temperature. ${ }^{57}$ This effect is typically seen in the range $700-800^{\circ} \mathrm{C}$ as the growth rate decreases rapidly for a given set of fluxes. It was proposed that the increased Ga desorption from the surface at these temperatures could be responsible. $^{26,58}$ The rates for desorption and decomposition follow an Arrhenius temperature dependence:

$$
\text { Rate }=A e^{\frac{-E_{a}}{k_{B} T}}
$$

where $E_{a}$ is the activation energy of the process, $k_{B}$ is the Boltzmann constant, $T$ is the temperature, and $\mathrm{A}$ is a constant. GaN decomposition has been shown to proceed with an activation energy of $3.1 \mathrm{eV}$ and a pre-exponent constant of $5 \times 10^{28} \mathrm{~cm}^{-2} \mathrm{sec}^{-1}{ }^{59}$ The activation energy for $\mathrm{Ga}$ desorption is $2.7 \mathrm{eV}$ with a pre-factor of $1 \times 10^{28} \mathrm{~cm}^{-2} \mathrm{sec}^{-1}{ }^{60}$ Since Ga desorption may play a significant role, the temperature dependent growth rate decrease expected if the only contributing factor was the increased Ga-desorption is also shown in Figure 3.13. This trend is consistent with the above mentioned RHEED results which indicated a rapidly increasing Ga-flux is required to maintain Ga-stabilized conditions for rf-plasma MBE growth above $700^{\circ} \mathrm{C}$. However, this is only true for Gapolar growth, and the decrease in growth rate is seen on both polarities. Additionally, Ga-desorption cannot be the only effect, as increasing the Ga overpressure does not overcome the decreased growth rate for a given temperature. ${ }^{24,57,61}$

The relative growth rate vs temperature is shown for two operating conditions of the EPI source. For Ga-stable conditions and growth rates comparable to the Oxford 


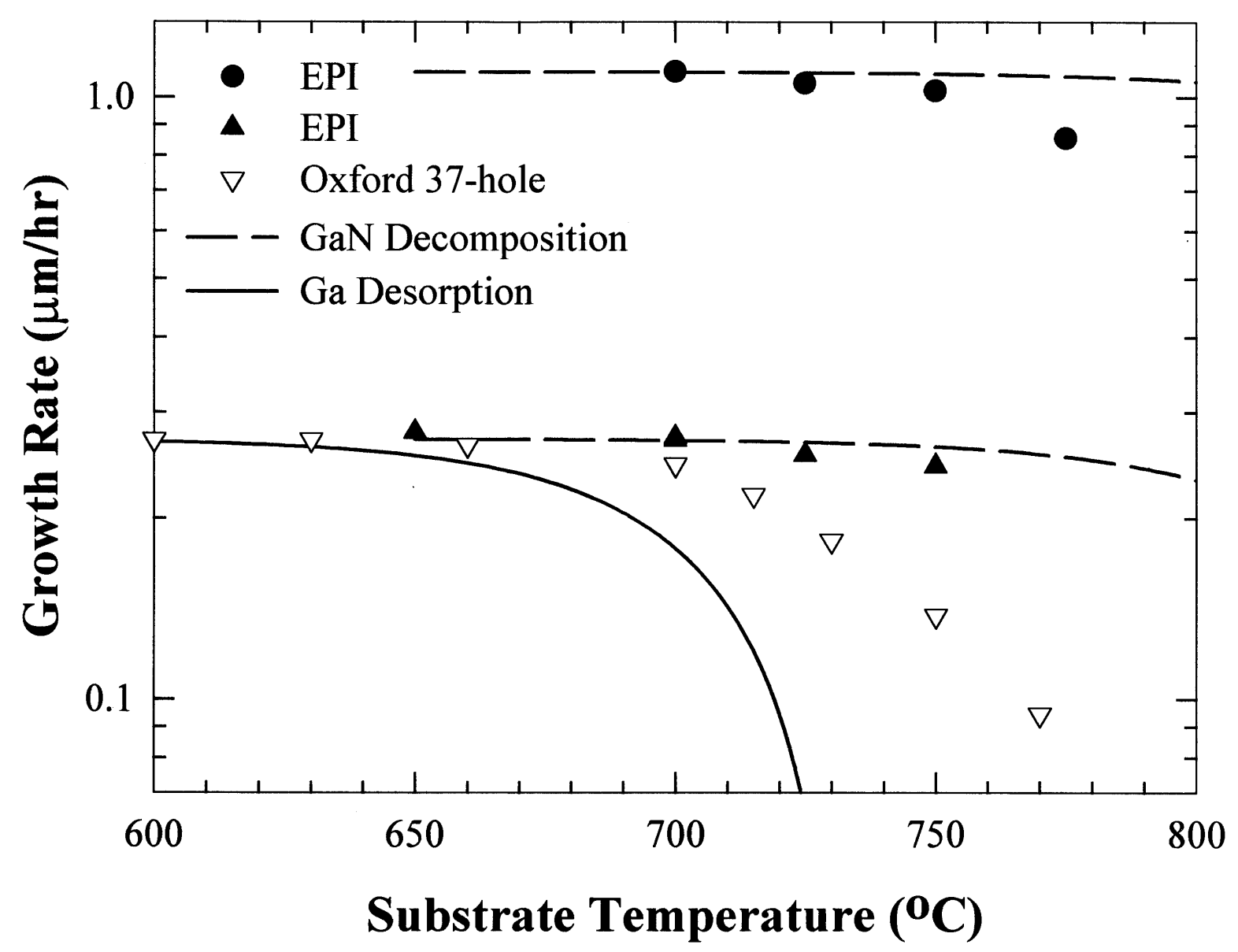

Figure 3.13 Relative growth rates of GaN for various conditions. Growth with molecular nitrogen metastables brings the growth rate vs temperature curve more in line with predictions from decomposition rates. 
source, the decrease in growth rate is now shifted to a higher temperature. A similar trend is observed at a growth rate of $1.0 \mu \mathrm{m} / \mathrm{hr}$. It is important to note that this latter growth rate was unattainable with the Oxford source. Shown for comparison is the dependence of growth rate on temperature expected if the only contributing factor was GaN decomposition. ${ }^{58}$ The decrease in growth rate is now comparable to the decomposition rate and is similar to that reported for ammonia-based MBE. ${ }^{24,62}$ The results with the EPI source indicate that Ga desorption is not the dominant limiting factor, and that the role of active nitrogen species must be taken into account.

Table 3.2 contains a subset of possible reactions occurring during Ga stable growth. The first column lists reactions leading to growth, while the second column details reactions in competition with growth. Of particular relevance is that ionic and neutral atomic nitrogen can participate in both the growth and in the decomposition of GaN. While rate constants for the reactions are not well known, there is a significant driving force for decomposition based on free energy considerations ${ }^{63}$ that may explain the relatively poor efficiency for growth with atomic nitrogen indicated in Table 3.1. Thus, competition between growth, surface decomposition, and adsorbed nitrogen capture may limit the growth efficiency of atomic nitrogen. Such a situation would promote point defect formation, supported by the poor electrical properties discussed below. The decrease in growth rate observed at $775^{\circ} \mathrm{C}$ for the EPI source may also be related to the residual atomic nitrogen flux in that source.

Both atomic and metastable molecular nitrogen contain significantly more energy than required for GaN formation. ${ }^{63}$ It costs approximately $2 \mathrm{eV}$ to form a GaN bond, but metastable molecules have about $6 \mathrm{eV}$ of energy available, and atoms have even more. This extra energy must go somewhere as the GaN is formed. An interesting scenario ${ }^{64}$ proposes that incorporation of atomic nitrogen releases this energy into the lattice where it can drive unfavorable reactions. This extra energy can contribute to point defect formation and can have a dramatic effect on the quality of the crystal. The excited molecule, however, can incorporate one atom into the growing $\mathrm{GaN}$ layer while the other desorbs, carrying away the excess energy. 
Table 3.2. Possible mechanisms occurring during the growth of GaN.

\begin{tabular}{cc}
\hline \hline Growth & Competition to Growth \\
\hline$G a+\frac{1}{2} N_{2}^{+}+e^{-} \rightarrow G a N$ & $G a N \rightarrow G a \uparrow+\frac{1}{2} N_{2} \uparrow$ \\
$G a+\frac{1}{2} N_{2}^{*} \rightarrow G a N$ & $G a_{\text {adsorbed }} \rightarrow G a \uparrow$ \\
$G a+N \rightarrow G a N$ & $G a N+N \rightarrow G a+N_{2} \uparrow$ \\
$G a+N^{+}+e^{-} \rightarrow G a N$ & $G a N+N^{+}+e^{-} \rightarrow G a+N_{2} \uparrow$ \\
\hline \hline
\end{tabular}




\subsubsection{Desorption Mass Spectroscopy Measurements}

As mentioned previously, DMS can be used to study the growth kinetics of GaN, such as the adsorption of $\mathrm{Ga}$ and the effects on growth of atomic hydrogen. DMS was used to monitor the desorbed Ga-flux during growth with nitrogen metastables using the EPI source. These measurements were only possible for growth of N-polar GaN. There was a constant signal of desorbing Ga during Ga-stable growth that made measurements of small flux changes impossible. For N-polar growth, reduction of the desorbed Ga-flux to values less than the minimum detectable flux of $0.3 \times 10^{14} \mathrm{~cm}^{-2} \mathrm{~s}^{-1}$ led to a switch from Ga-stable to N-stable growth. This change is in agreement with the change in surface morphology indicated by the RHEED pattern switching from a streaky, two-dimensional pattern to a spotty pattern characteristic of three-dimensional growth. In general, Ga-stable conditions were maintained during growth with a desorbed Ga-flux between 0.5 and $1 \times 10^{14} \mathrm{~cm}^{-2} \mathrm{~s}^{-1}$. For a constant flux of both $\mathrm{N}$ and $\mathrm{Ga}$, an approximate $20 \%$ increase in the desorbing Ga-flux was observed between 700 and $780^{\circ} \mathrm{C}$. While this increase in $\mathrm{Ga}$ desorption correlates well with the observed decrease in growth rate, the observed increase in desorption is significantly less than expected from the rate indicated in Figure 3.13. This gives further evidence that while Ga-desorption plays a role in GaN growth, it is not a significant limitation for Ga-stable growth at these temperatures using nitrogen metastables.

\subsubsection{Optical and Electrical Measurements}

Hall effect measurements are shown in Figure 3.14(a) comparing the electrical properties of GaN grown using the Oxford and EPI sources. A significant increase in carrier mobility is observed for growth with the molecular nitrogen metastables found in the EPI source. A considerable decrease in the associated carrier concentration is shown in Figure 3.14(b) for the metastable molecular nitrogen flux. The EPI results are consistent with improved electrical properties that have also been observed by other groups using a similar source configuration. ${ }^{45,65,66}$ The electrical results for the Oxford source, however, are comparable to most early values reported for rf-plasma MBE. The improvement in material quality using nitrogen metastables is also seen in photoluminescence measurements. Several undoped samples have shown free excitonic 


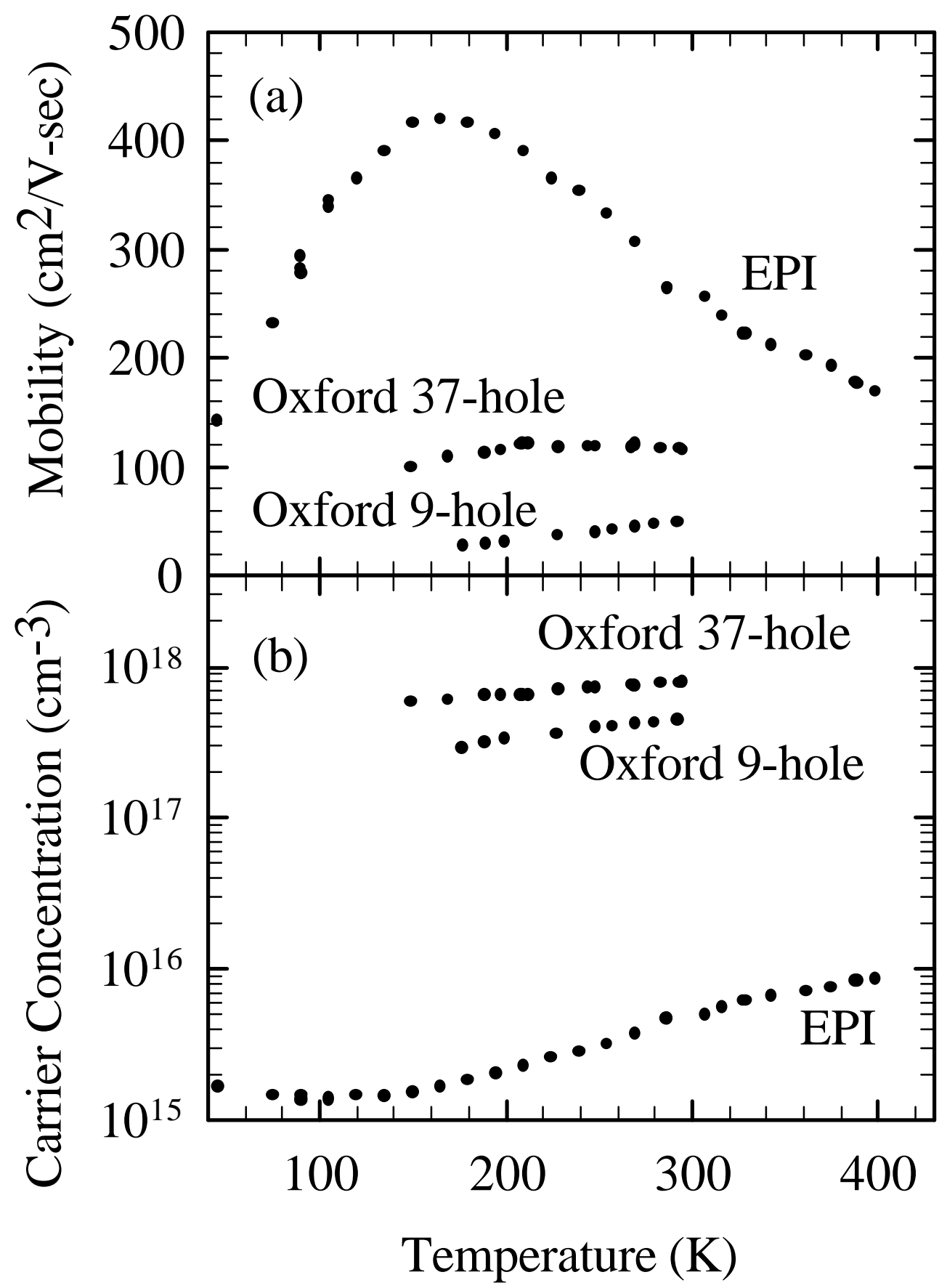

Figure 3.14 Comparison of the electrical properties of GaN grown with the Oxford and EPI plasma sources. The results for the Oxford source are for samples grown under atomic hydrogen. 
luminescence, as seen in Figure 3.15, indicative of high quality material. Our current study indicates that growth with predominantly atomic nitrogen may result in a significant concentration of point defects limiting layer quality. Indeed, our highest mobility values with the Oxford source were obtained for growth under an atomic hydrogen flux, which may stabilize the growing surface. ${ }^{57}$ Although lower carrier concentrations than those shown could be obtained with the Oxford source for growth without hydrogen, the accompanying mobilities were also significantly smaller.

\subsubsection{Effects of Atomic Hydrogen}

The dramatic effect of atomic hydrogen on the growth of GaN by rf-plasma MBE reported in previous studies ${ }^{55,57}$ can now be better understood, at least for N-polarity growth. A bulk terminated surface would have a nitrogen dangling bond at the surface. This is normally accommodated by the formation of a surface Ga adlayer, as described by Smith et al. ${ }^{67}$ The dangling bond, however, would make this surface more prone to decomposition through attack by atomic nitrogen. The rapid decrease in growth rate with increasing temperature may be related to the thermal activation of this reaction. If atomic hydrogen is available, it can also attach to the dangling bond thereby passivating the surface. The presence of the N-H bond would prevent the enhanced decomposition due to attack by atomic nitrogen. This scenario is supported by the recovery of the growth rate for the Oxford source when using atomic hydrogen as shown in Figure 3.13. At the highest temperatures investigated, growth with atomic hydrogen results in a growth rate about a factor of three times larger than growth without atomic hydrogen. Growth under atomic hydrogen using the Oxford source appeared to be $\mathrm{N}$ stable as indicated by a spotty three-dimensional RHEED pattern, Ga limited growth rates, ${ }^{57,61}$ and the growth characteristics of inversion domains. ${ }^{68}$ The recovery to the $0.3 \mu \mathrm{m} / \mathrm{hr}$ growth rate shown in Figure 3.13 is indicative of the Ga-limited nature coupled with the fact that the Ga flux was kept constant for this comparison. Increasing the Ga flux lead to steadily increasing growth rates until $\mathrm{Ga}$ condensation occurred at the temperatures investigated, to a maximum growth rate of $0.5 \mu \mathrm{m} / \mathrm{hr}$ in our study. However, the electrical properties of these films remained poor in comparison with the EPI source. 


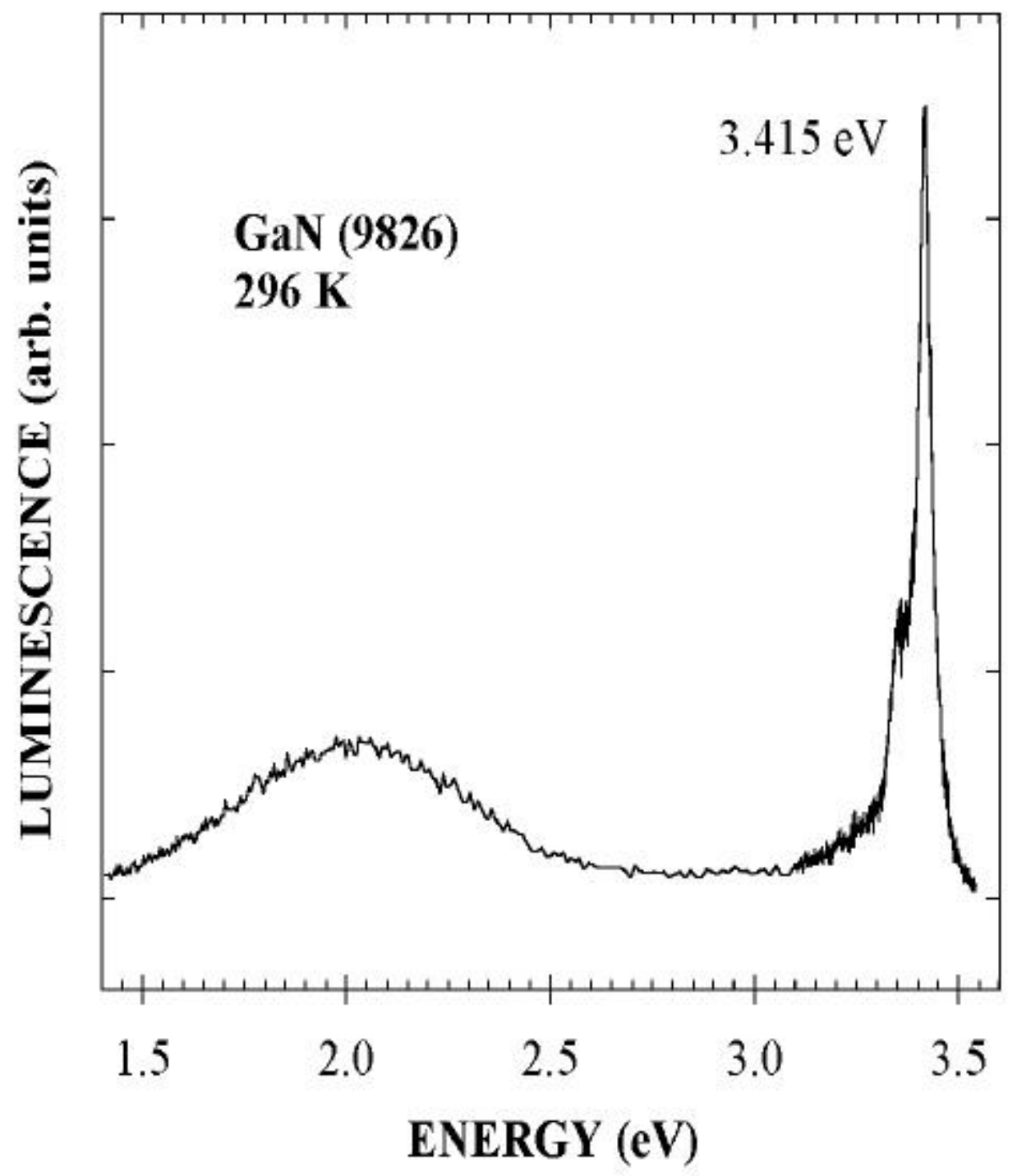

Figure 3.15 Room temperature photoluminescence measurement of undoped $\mathrm{GaN}$ showing a strong free excitonic recombination. This is indicative of high quality material. 
The effect of atomic hydrogen on growth rate with the EPI source was significantly different, likely reflecting the difference in the predominant active nitrogen specie. Rather than recovering the growth rate for a fixed Ga flux, as was seen during high temperature growth with the Oxford source, an increase in the growth rate was observed for the same growth conditions as the $0.3 \mu \mathrm{m} / \mathrm{hr}$ case, as indicated by Figure 3.13. Interestingly, the increase at the lower temperature was approximately the same magnitude as the measured atomic nitrogen flux. Atomic hydrogen affected growth rates less at higher temperatures, with very little difference at the highest temperature investigated. Figure 3.16 contains the desorbed Ga flux observed for growth under atomic hydrogen. Since the same source conditions were used as the $0.3 \mu \mathrm{m} / \mathrm{hr}$ case, the reduced $\mathrm{Ga}$ desorption reflects the increase in growth rate. Increasing the substrate temperature led to a larger increase in desorption rate than for growth without atomic hydrogen.

The effect of increasing the atomic hydrogen flux for fixed growth conditions is shown in Figure 3.17. Here, the base conditions lead to a GaN growth rate of about $1.6 \mu \mathrm{m} / \mathrm{hr}$. The addition of an atomic hydrogen flux increased the rate to more than $2 \mu \mathrm{m} / \mathrm{hr}$. Further increase in the atomic hydrogen flux then led to a steadily decreasing growth rate. The beam equivalent pressures shown reflect the total hydrogen flux, not just that of atomic hydrogen. The substrate temperature is large enough for N-H bond breaking coupled with hydrogen desorption, and so any "passivation" effect must be dynamic. The interaction of atomic hydrogen with the growing $\mathrm{GaN}$ surface is a complex situation. A relatively low concentration may protect the growing surface from attack by atomic nitrogen, while at higher concentrations the atomic hydrogen begins to compete with $\mathrm{Ga}$ for nitrogen bonds, resulting in a higher probability for Ga desorption. In addition, there is some evidence that hydrogen may even enhance the surface decomposition of $\mathrm{GaN}$ at higher temperatures, such as those used for MOCVD growth. ${ }^{69,70}$ These results indicate that the effect of atomic hydrogen is certainly complex and must be considered when trying to understand the growth of GaN. 


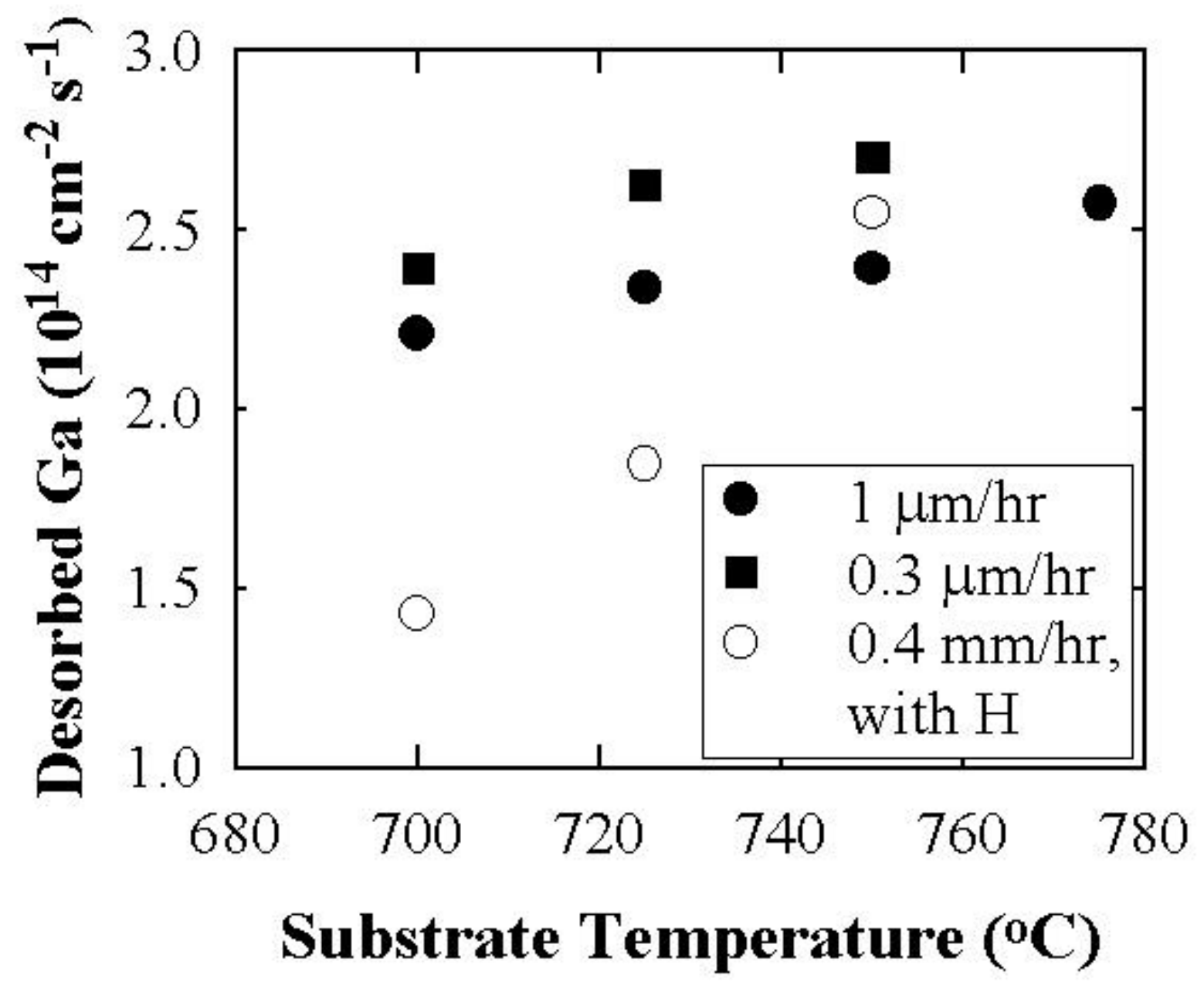

Figure 3.16 Desorbed Ga flux vs. temperature for two different growth rates and growth under an atomic hydrogen flux. 


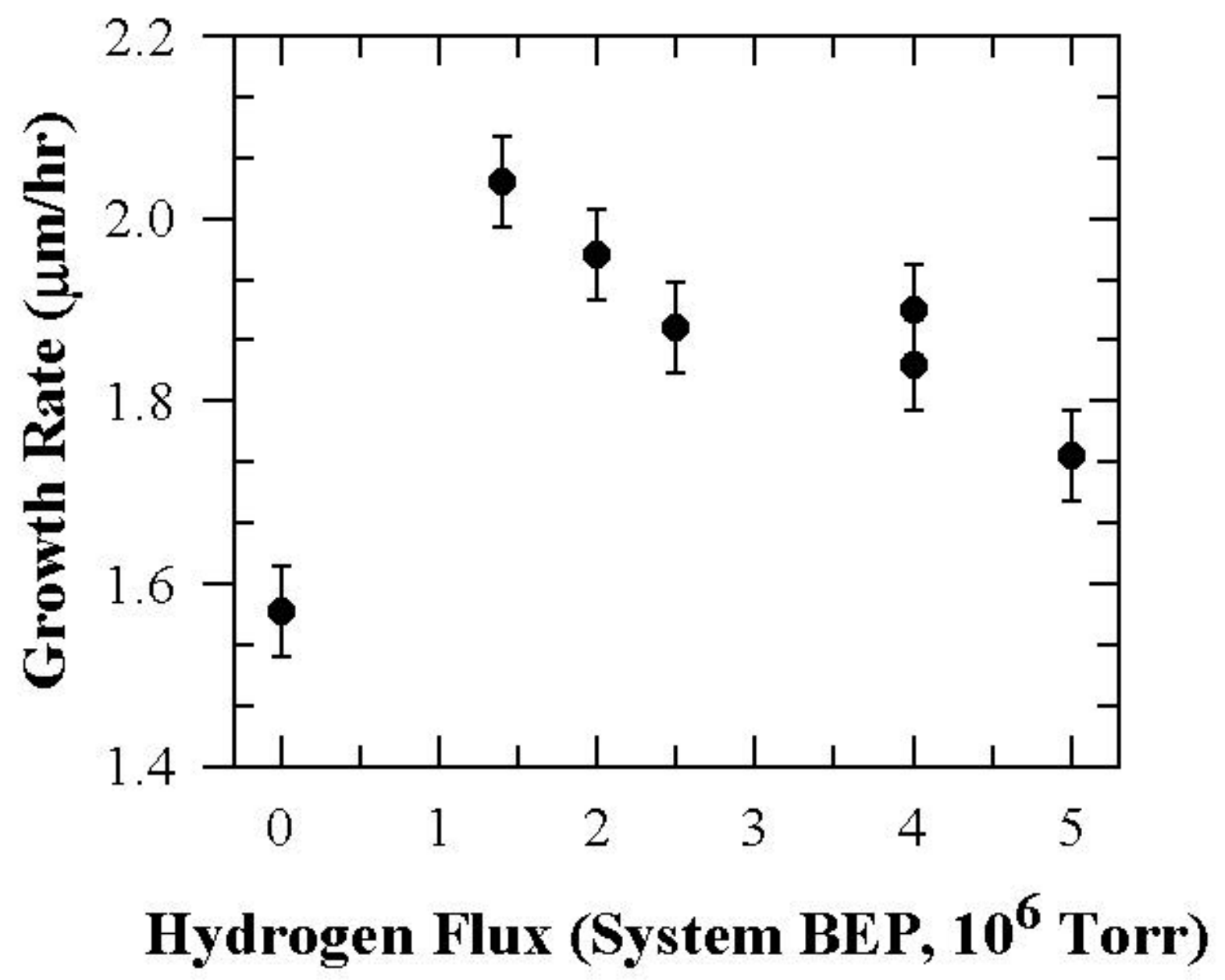

Figure 3.17 Effect of atomic hydrogen on the growth rate of GaN when using the EPI source. 


\subsection{Summary}

Two rf-plasma sources were studied to determine the reactivity of various types of active nitrogen. The flux from rf-plasma sources is a complex mixture of ionic and neutral atomic and molecular species. The Oxford CARS-25 source, in conjunction with an electrostatic deflector plate, could provide an active nitrogen flux consisting of high energy ionic molecular and atomic nitrogen, a mixture of atomic nitrogen and lower energy ions, or predominantly atomic nitrogen. The EPI Unibulb source produced a significant amount of metastable molecular nitrogen. The nitridation rate for high-energy ions was $\sim 0.3 \AA / \mathrm{min}$ for the first $10 \AA$, about three times faster than nitridation by atomic nitrogen at a similar concentration. The mixed atomic nitrogen/low energy ion condition showed an initiation stage that lasted for $\sim 180$ minutes, followed by nitridation at a rate of $0.03-0.04 \AA / \mathrm{min}$. This initiation stage may be due to formation and desorption of molecular nitrogen from the sapphire surface. Metastable nitrogen does not appear to be very reactive for nitridation, but is effective for the growth of group III-nitrides.

Boron contamination originating from the PBN liners of the plasma sources was observed. While it is not clear what role a small background of B has on the properties of $\mathrm{GaN}, \mathrm{B}$ may have a significant influence on the nitridation of sapphire using rf-plasma sources. In particular, the formation of $\mathrm{Al}_{1-\mathrm{x}} \mathrm{B}_{\mathrm{x}} \mathrm{N}$ alloys can explain observed lattice constants smaller than expected for AlN. Segregation of BN in large x-value alloys coupled with nucleation of the more thermodynamically stable cubic BN phase may explain one possible mode for the subsequent nucleation of cubic GaN or AlN on nitrided sapphire.

Studies of growth rate as a function of temperature suggest the GaN surface is prone to "attack" by neutral and ionic atomic nitrogen above $700^{\circ} \mathrm{C}$, promoting enhanced decomposition. Growth using neutral metastable molecular nitrogen results in a temperature-dependent growth rate similar to that of growth with ammonia. Hydrogen can be used to stabilize the growing surface, at least for N-polarity growth. Too much 
hydrogen may promote Ga desorption, possibly due to competition for $\mathrm{N}$ bonds. Growth with predominantly metastable nitrogen also resulted in improved electrical quality. Metastable molecular nitrogen is preferable to neutral or ionic atomic nitrogen for MBE growth. 


\section{Chapter 4: Magnesium in Gallium Nitride}

At present, the only technologically feasible p-type dopant for the III-N system is $\mathrm{Mg}$, which exhibits a relatively large acceptor activation energy of $\sim 200 \mathrm{meV} .^{13}$ Assuming this energy and a hole effective mass of $0.8 \mathrm{~m}_{0}$ in $\mathrm{GaN}$, only $1 \%$ of the $\mathrm{Mg}$ acceptors are electrically active at room temperature. Since so few of the acceptors are active, extremely high $\mathrm{Mg}$ concentrations are required to achieve the p-type conductivity necessary for many device applications. Further complications occur when $\mathrm{Mg}$ is used as the p-type dopant for the growth of GaN by MOCVD since electron irradiation ${ }^{71}$ or thermal annealing ${ }^{72}$ must be used to dissociate compensating $\mathrm{Mg}-\mathrm{H}$ complexes and activate the $\mathrm{Mg}$. In addition, $\mathrm{Mg}$ has a relatively high vapor pressure. A considerable amount of the $\mathrm{Mg}$ that has been deposited inside the vacuum system during previous growths will become gaseous at the high temperatures used in MOCVD growth (greater than $1000^{\circ} \mathrm{C}$ ). This $\mathrm{Mg}$ "memory effect" gives rise to broad doping profiles that make careful junction placement difficult, ${ }^{73}$ and leads to excessive amounts of $\mathrm{Mg}$ being incorporated into layers that are intended to be undoped.

$\mathrm{MBE}$ is an alternate growth technique that may ameliorate these problems. For example, since MBE can be a "hydrogen-free" growth process, high p-type activation in as-grown MBE GaN layers has been reported due to the lack of $\mathrm{Mg}-\mathrm{H}$ compensating bonds. $^{73}$ These same conditions also gave sharp doping interfaces. While there have been many studies of $\mathrm{Mg}$ doping during the $\mathrm{MBE}$ growth of $\mathrm{GaN},{ }^{44}$ many effects remain poorly understood. The significantly varying results are likely due to differences in growth conditions employed, which are not always clearly described in the literature. In addition, as pointed out by Li et al., ${ }^{74}$ both Ga-polar and N-polar GaN can be grown by MBE, depending on the nucleation conditions. The different surface polarities can yield vastly different results, with $\mathrm{Li}$ et al. obtaining p-type conduction with $\mathrm{Mg}$ only for the Ga-polarity. In contrast, Orton et al. ${ }^{75}$ have reported electrical measurements equivalent to Li et al.'s Ga-polar results for Mg-doped N-polar material (inferred from their growth conditions) obtained by growing under N-stable conditions. There are many other 
interesting phenomena related to $\mathrm{Mg}$ that cause it to be a non-ideal dopant. Among these are the significant temperature dependence of incorporation, ${ }^{76}$ surface segregation/accumulation effects, ${ }^{77}$ surfactant effects ${ }^{78}$ and the potential to cause Ga-to$\mathrm{N}$-polarity inversion. ${ }^{79}$ Each of these effects must be dealt with in order to achieve the requirements for p-type doping.

\subsection{Incorporation of Magnesium}

This study was initiated to facilitate the understanding of $\mathrm{Mg}$ as a p-type dopant during the MBE growth of GaN. It is necessary to understand how $\mathrm{Mg}$ is incorporated into the crystal during growth in order to understand the effects listed above. A number of $\mathrm{Mg}$ doped layers were grown under various growth conditions with that aim in mind. The results of measurements on these samples confirm that there are serious hurdles to be overcome for Mg-doping, and help to explain some of the more puzzling results from the literature.

\subsubsection{Growth Conditions}

The Mg-doped GaN layers in this study were grown by rf-plasma assisted MBE using the EPI Unibulb nitrogen plasma source described in Chapters 2 and 3. Incorporation of $\mathrm{Mg}$ was studied on both polarities of $\mathrm{GaN}$. N-polarity GaN was obtained by nucleating GaN buffer layers directly on sapphire under heavily Ga-rich conditions and incorporation in Ga-polarity GaN was studied by growth on MOCVD $\mathrm{GaN}$ templates grown on (0001) sapphire substrates. The doped layers were grown at a rate of $0.25 \mu \mathrm{m} / \mathrm{hr}$, which corresponds to a nitrogen flow rate of $0.85 \mathrm{sccm}$ and rf-power of $200 \mathrm{~W}$. The samples were grown under Ga-stable conditions $(\mathrm{Ga} / \mathrm{N}$ flux ratio greater than 1) that result in high quality GaN growth. ${ }^{19,23,61} \mathrm{Mg}$ step-doped structures were produced by opening and closing the Mg shutter. In-situ growth rates were monitored using the laser reflectance technique described in Chapter 2. All changes in oven and substrate temperatures occurred with the Mg shutter closed. 


\subsubsection{Dependence of Magnesium Incorporation on Surface Polarity}

Figure 4.1 contains the SIMS measurement of Mg made on a N-polarity Mg stepdoped structure, and Figure 4.2 contains a similar scan for a Ga-polar sample. The general features observed here for both samples are representative of all the $\mathrm{Mg}$ structures investigated. Several features are immediately obvious. First, at the lower $\mathrm{Mg}$ oven temperatures and higher substrate temperatures there is no evidence of $\mathrm{Mg}$ incorporation. It does appear that $\mathrm{Mg}$ may start to incorporate for an oven temperature of $250^{\circ} \mathrm{C}$ and a substrate temperature of $650^{\circ} \mathrm{C}$, but at a gradually increasing rate rather than an abrupt turn-on. Significant $\mathrm{Mg}$ is seen to incorporate at larger $\mathrm{Mg}$ flux, with strong substrate temperature dependence as reported previously. ${ }^{76}$

Our results also indicate a strong dependence of $\mathrm{Mg}$ incorporation on polarity for identical growth conditions. In particular, at higher $\mathrm{Mg}$ flux and higher temperatures, $\mathrm{Mg}$ incorporation is found to be approximately fifteen to twenty-five times less in N-polar GaN. A significant difference was observed for each set of comparable conditions examined, with larger incorporation always occurring for the Ga-polarity. A summary of the results measured for all the Mg step-doped structures is given in Table 4.1. From this table, it is clear that $\mathrm{Mg}$ incorporates at a higher rate for Ga-polarity growth than for the N-polarity.

This observation is consistent with the lower electrical activation observed for N-polar growth compared to Ga-polar growth observed by Li et al. ${ }^{74}$ The fact that p-type conduction was only seen for Ga-polar growth is easily explained by the larger incorporation of $\mathrm{Mg}$ for this surface polarity. In this case, $\mathrm{Mg}$ incorporation for $\mathrm{N}$-polar growth (which is less by more than an order of magnitude) may not be high enough to overcome the existing n-type background. Such samples would be highly resistive, or indeed, n-type due to compensation.

\subsubsection{The Effects of Atomic Hydrogen}

Calculations by Neugebauer and Van de Walle ${ }^{80}$ suggested that the presence of hydrogen would enhance Mg incorporation. The results shown in Figures 4.1 and 4.2 


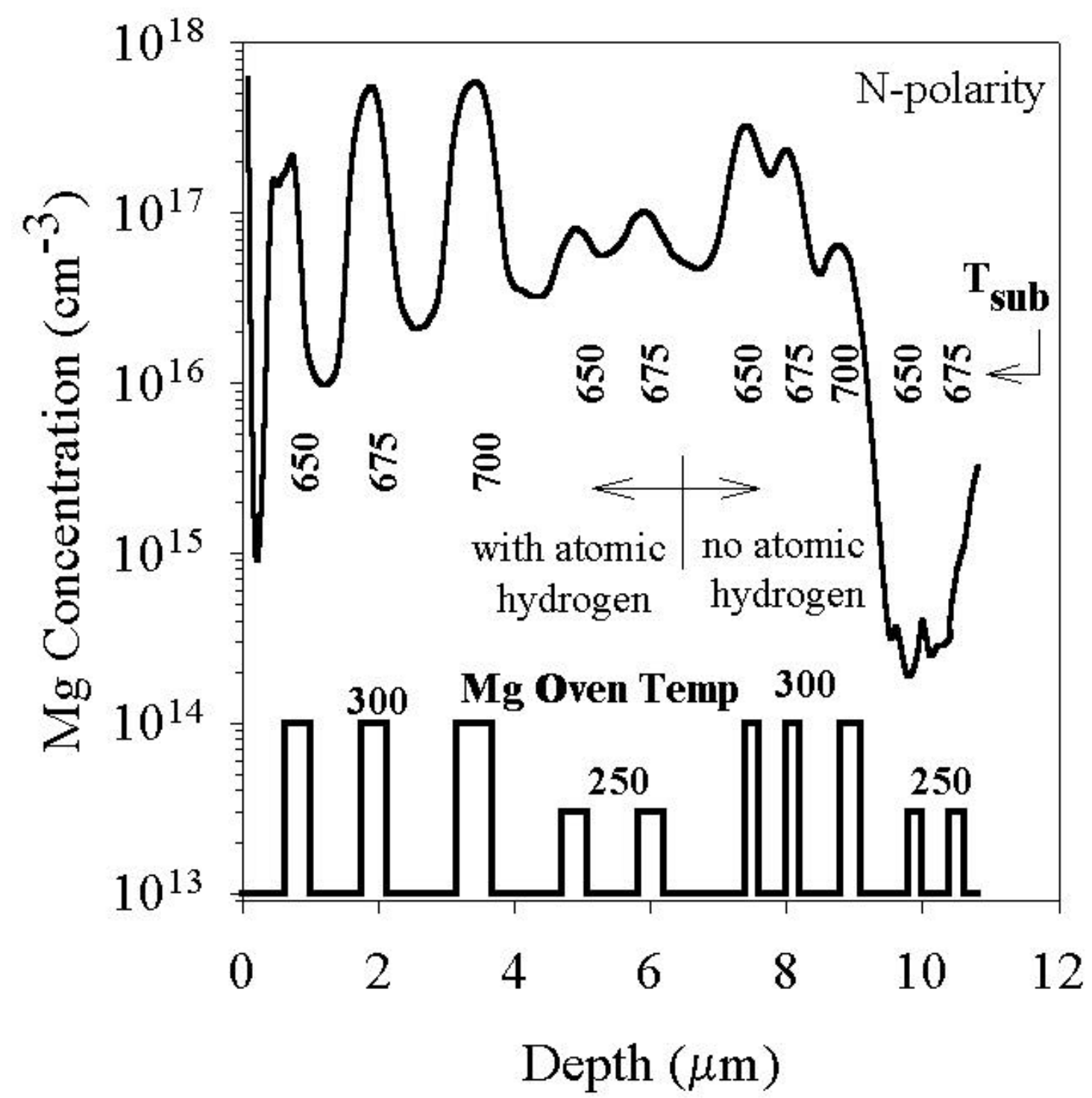

Figure 4.1 SIMS measurement of Mg incorporation in a N-polar film grown under various conditions. Shown schematically on the bottom of the figure are the opening and closing positions of the Mg shutter, as well as the Mg furnace temperatures. 


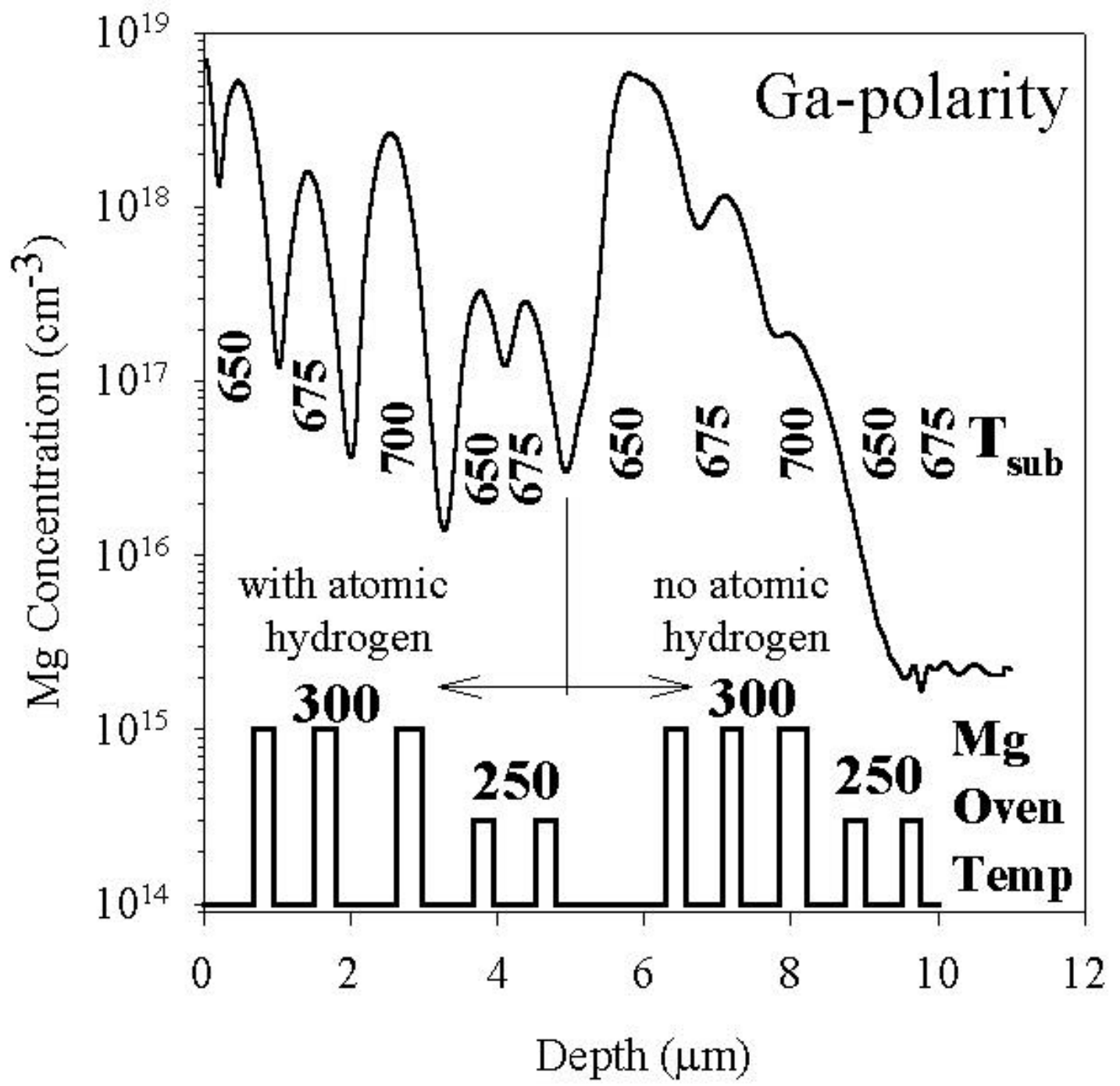

Figure 4.2 SIMS measurement of Mg incorporation in a N-polar film grown under various conditions. Shown schematically on the bottom of the figure are the opening and closing positions of the Mg shutter, as well as the $\mathrm{Mg}$ furnace temperatures. 
Table 4.1 SIMS results for all Mg step-doped samples investigated.

\begin{tabular}{|c|c|c|c|c|c|c|c|}
\hline \multirow[t]{2}{*}{$\mathbf{T}_{\text {sub }}$} & \multirow[t]{2}{*}{$\mathbf{T}_{\mathbf{M g}}$} & \multirow[t]{2}{*}{ Polarity } & \multirow[t]{2}{*}{ [Mg] } & \multirow[t]{2}{*}{ [Mg]:H } & \multirow{2}{*}{$\begin{array}{c}\text { [Mg] Ratio } \\
\text { with/ w/out } H\end{array}$} & \multicolumn{2}{|c|}{$\begin{array}{c}\mathrm{Mg}] \text { Ratio } \mathrm{Ga} / \mathrm{N} \\
\text { polarity }\end{array}$} \\
\hline & & & & & & w/out $\mathrm{H}$ & with $\mathrm{H}$ \\
\hline$\overline{7705}$ & 250 & $\overline{\mathrm{N}}$ & $\overline{\mathrm{ND}}$ & ND & & & \\
\hline 705 & 300 & $\mathrm{~N}$ & $1.0 \times 10^{18}$ & $2.0 \times 10^{18}$ & 2.0 & & \\
\hline 705 & 350 & $\mathrm{~N}$ & $4.5 \times 10^{18}$ & $6.0 \times 10^{18}$ & 1.3 & & \\
\hline 705 & 400 & $\mathrm{~N}$ & $8.0 \times 10^{18}$ & $1.0 \times 10^{19}$ & 1.3 & & \\
\hline 700 & 350 & $\mathrm{Ga}$ & $7.0 \times 10^{19}$ & $1.0 \times 10^{20}$ & 1.4 & 15.6 & 16.7 \\
\hline 725 & 350 & $\mathrm{Ga}$ & $9.0 \times 10^{18}$ & $2.3 \times 10^{19}$ & 2.6 & & \\
\hline 710 & 350 & $\mathrm{Ga}$ & $7.1 \times 10^{19}$ & & & & \\
\hline 650 & 250 & $\mathrm{~N}$ & ND & $8.1 \times 10^{16}$ & $>200$ & & \\
\hline 675 & 250 & $\mathrm{~N}$ & $\mathrm{ND}$ & $1.0 \times 10^{17}$ & $>250$ & & \\
\hline 650 & 300 & $\mathrm{~N}$ & $3.3 \times 10^{17}$ & $2.1 \times 10^{17}$ & 0.6 & & \\
\hline 675 & 300 & $\mathrm{~N}$ & $2.3 \times 10^{17}$ & $5.4 \times 10^{17}$ & 2.3 & & \\
\hline 700 & 300 & $\mathrm{~N}$ & $6.5 \times 10^{16}$ & $5.9 \times 10^{17}$ & 9.1 & & \\
\hline 650 & 250 & $\overline{\mathrm{Ga}}$ & $\mathrm{ND}$ & $3.3 \times 10^{17}$ & $>165$ & & 4.1 \\
\hline 675 & 250 & $\mathrm{Ga}$ & $\mathrm{ND}$ & $2.8 \times 10^{17}$ & $>140$ & & 2.8 \\
\hline 650 & 300 & $\mathrm{Ga}$ & $5.8 \times 10^{18}$ & $5.3 \times 10^{18}$ & 0.9 & 17.6 & 25.2 \\
\hline 675 & 300 & $\mathrm{Ga}$ & $1.1 \times 10^{18}$ & $1.5 \times 10^{18}$ & 1.4 & 4.8 & 2.8 \\
\hline 700 & 300 & $\mathrm{Ga}$ & $1.9 \times 10^{17}$ & $2.7 \times 10^{18}$ & 14.2 & 2.9 & 4.6 \\
\hline
\end{tabular}


and shown more explicitly in Table 4.1 verify this prediction. In particular, without hydrogen, no incorporation was observed for low $\mathrm{Mg}$ flux. The addition of atomic hydrogen caused incorporation at levels more consistent with projections from higher Mg-flux incorporation rates. The effect of hydrogen on $\mathrm{Mg}$ incorporation was less pronounced at higher $\mathrm{Mg}$ flux or lower substrate temperature. Indeed, the atomic hydrogen has little effect or may slightly inhibit incorporation at $650{ }^{\circ} \mathrm{C}$ for reasonable $\mathrm{Mg}$ flux. The presence of atomic hydrogen appeared to sharpen interfaces although diffusion effects are also present in our samples. However, the profiles indicate that surface accumulation layers are forming, as discussed below. This suggests that the increase in sharpness is due to enhanced incorporation as opposed to the suppression of surface accumulation. Note also that while hydrogen enhances incorporation for both polarities, it does not eliminate the polarity dependence of the magnitude of $\mathrm{Mg}$ incorporation.

Importantly, and in contrast to growth by MOCVD, SIMS on the Mg-doped layers grown under atomic hydrogen indicated that while hydrogen has a significant effect on $\mathrm{Mg}$ incorporation, hydrogen itself is not incorporated at significant levels. Hydrogen levels stayed at least an order of magnitude lower than the concentration of $\mathrm{Mg}$. During MOCVD growth, $\mathrm{Mg}$ and $\mathrm{H}$ incorporate in a 1:1 ratio. That is, if hydrogen is incorporated at the growing surface in our samples, it anneals out during growth at the temperatures used. In this way, hydrogen may be used during the MBE growth of GaN to increase the incorporation of $\mathrm{Mg}$, while still producing as-grown p-type material.

\subsection{Surface Accumulation/Segregation}

\subsubsection{Evidence for Surface Accumulation/Segregation}

During the analysis of the Mg SIMS data, it became necessary to determine the exact times (and hence depth in the sample) that the Mg shutter was opened or closed. This has already been seen schematically in Figures 4.1 and 4.2. The exact placement of these events was made possible by the laser reflectance growth rate measurements described in Chapter 2. The MBE/MOCVD interface was clearly identified by "chemical 
markers" seen in SIMS measurements such as a spike in Si at the film/substrate interface or the appearance of boron from the rf-plasma source liner in the MBE grown layer. The estimated error in placement is less than $0.1 \mu \mathrm{m}$.

Figure 4.3 shows that the incorporation of $\mathrm{Mg}$ is out of phase with the opening and closing of the shutter. The Mg concentration generally increases while the shutter is open, consistent with the observations of Orton et al. ${ }^{75}$ This is shown much more clearly in Figure 4.4 for $\mathrm{Mg}$ incorporation on both polarities. Importantly, significant amounts of $\mathrm{Mg}$ are incorporated after the $\mathrm{Mg}$ shutter was closed which is strongly indicative of surface segregation and accumulation of $\mathrm{Mg}$. Cheng et al. ${ }^{77}$ report evidence for surface accumulation of $\mathrm{Mg}$ for $\mathrm{Mg}$-doped $\mathrm{GaN}$ based on Auger spectroscopy of layers after growth. Ramachandran et al. ${ }^{78}$ report a significant persistence of the surfactant effect of $\mathrm{Mg}$ after closing the $\mathrm{Mg}$ shutter, indicating that $\mathrm{Mg}$ remains on the surface in the absence of a Mg flux. A final supporting piece of evidence is the high concentration of $\mathrm{Mg}$ seen at the surface of the sample during SIMS measurements. Typically, the first few data points of a SIMS measurement are ignored while waiting for steady-state conditions to be achieved. For these Mg-doped layers, however, the high measured $\mathrm{Mg}$ concentration persisted past these data points and is probably not measurement-related. It should be noted that about $0.25 \mu \mathrm{m}$ of undoped material was grown on top of the last $\mathrm{Mg}$ doped step in these samples. This is a further indication that $\mathrm{Mg}$ rides on the surface well after the shutter is closed.

For the Ga-polar growth shown in Figure 4.4, numerically summing the number of $\mathrm{Mg}$ atoms that incorporate after the shutter is closed shows that approximately $3.3 \times 10^{14} \mathrm{Mg}$ atoms $/ \mathrm{cm}^{2}$ were on the surface (assuming all of the surface atoms do eventually incorporate). This number corresponds to between $1 / 4$ and $1 / 2$ monolayer of $\mathrm{Mg}$ on the surface of the growing layer. Currently, there are two models that predict stable surface configurations of $\mathrm{Mg}$ on the GaN surface. Calculations by Bungaro et al. ${ }^{81}$ indicate that $1 / 4$ monolayer of $\mathrm{Mg}$ should be the most energetically favorable. This structure would have two electrons to fill dangling-bond derived surface states such that a surface gap could form. A surface energy gap would decrease the diffusion barrier, a 


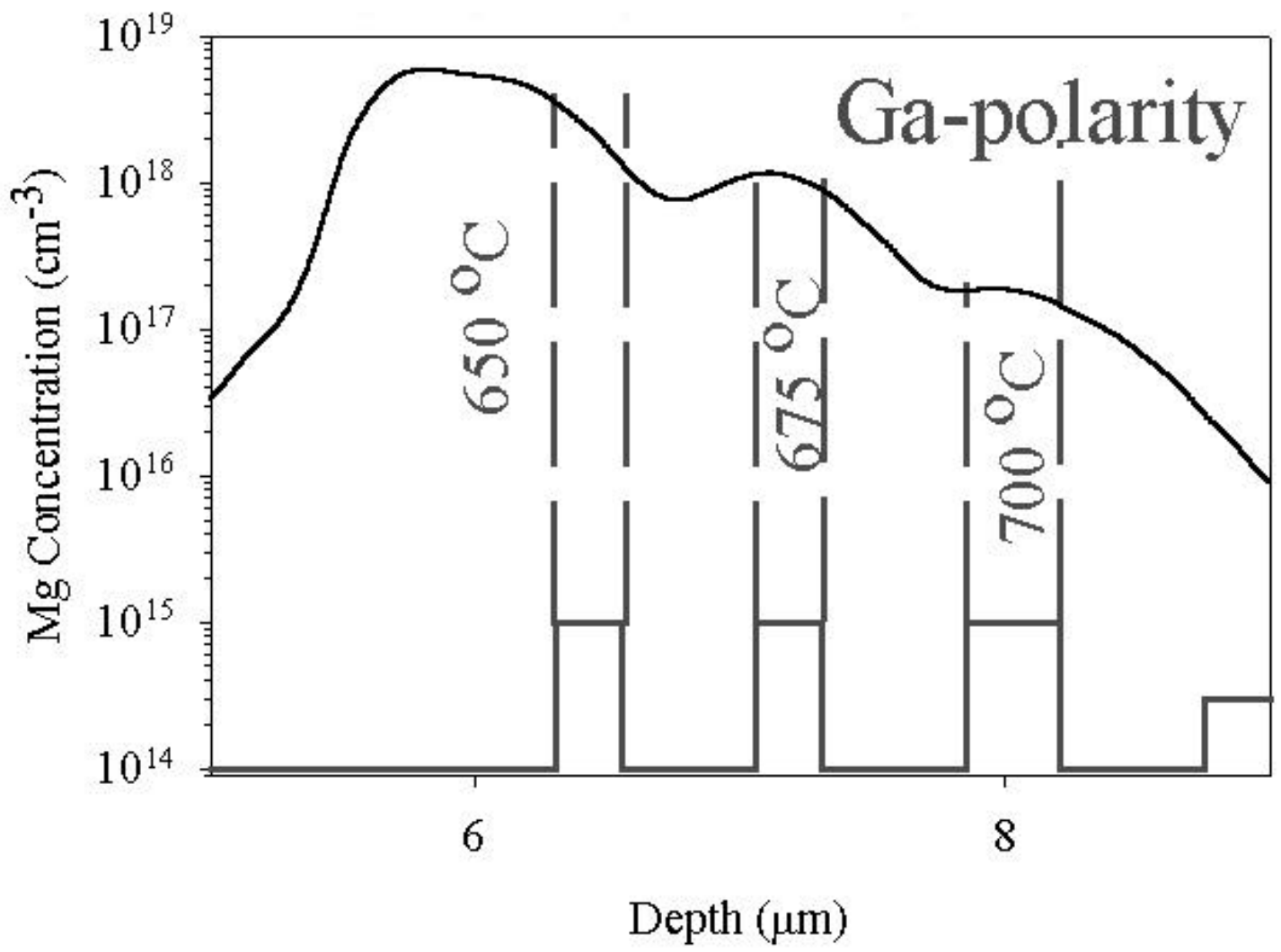

Figure 4.3 SIMS measurement of a Mg step-doped layer showing clear signs of surface accumulation. The opening and closing of the Mg shutter is indicated and the substrate temperatures are listed for each peak. 

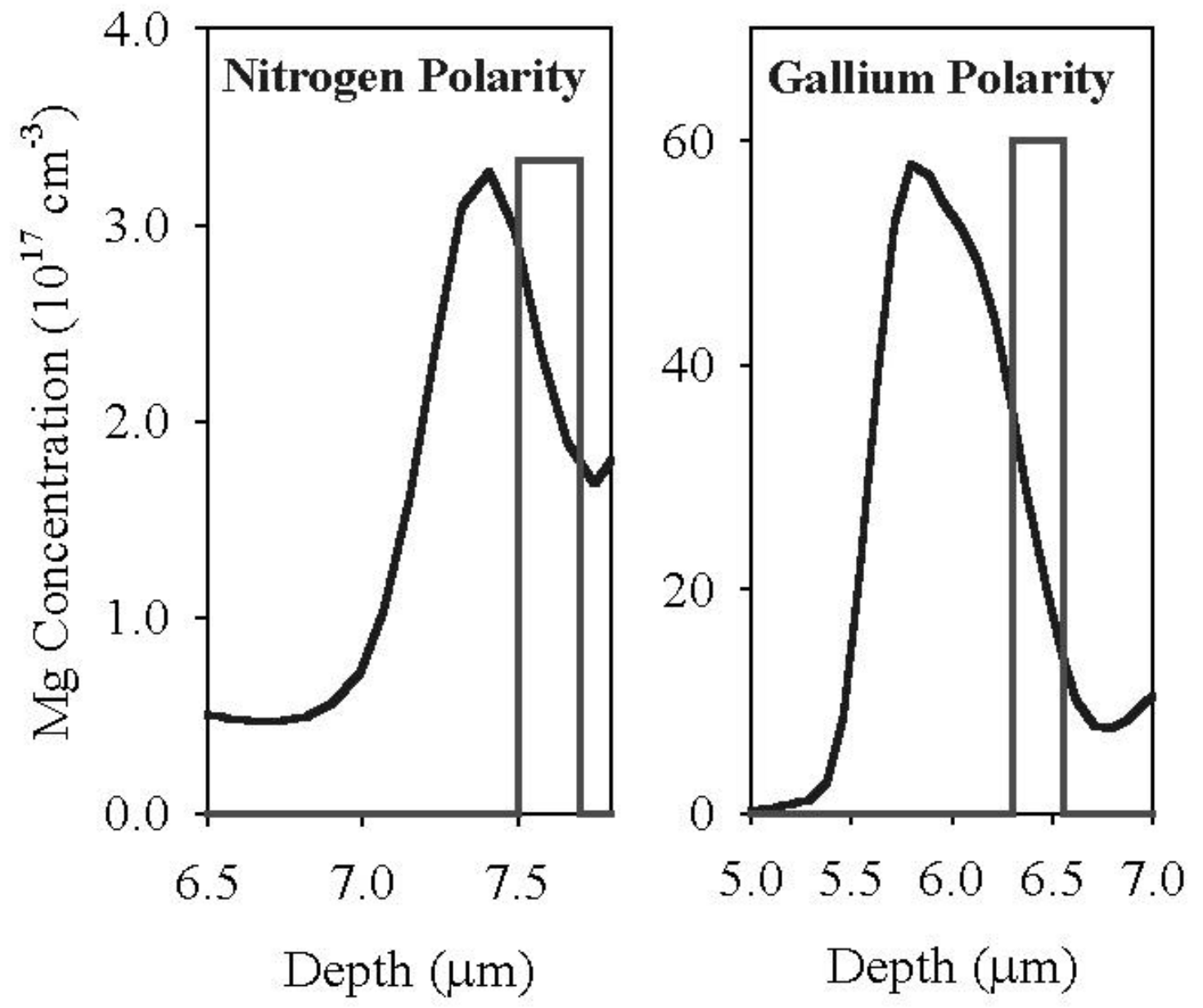

Figure 4.4 SIMS measurement of Mg showing surface accumulation on both polarities of GaN. The region where the $\mathrm{Mg}$ shutter was open is also shown. 
consequence of which is the possibility of a surfactant effect. In contrast, Ramachandran et al. ${ }^{79}$ propose that $3 / 4$ of a monolayer of $\mathrm{Mg}$ is the stable surface configuration based on total energy computations and electron counting arguments. Since there are no surface dangling bonds in this configuration, the surface should be fairly non-reactive and a low barrier to diffusion would also be expected, again consistent with the observed surfactant effect. Ramachandran et al. concede that their experimental results could be consistent with either model, but find that the $3 / 4$ monolayer model is energetically more favorable for Mg-rich or Ga-deficient conditions. The accuracy of our surface concentrations from SIMS results are not high enough to prove or disprove either model.

There are approximately $1 \times 10^{15}$ sites available per $\mathrm{cm}^{2}$ on the GaN surface. By summing the atoms that are incorporated after the shutter is closed, coupled with the knowledge of the bulk concentration from Figure 4.4, we find that approximately $3 \times 10^{-4}$ of the $\mathrm{Mg}$ incorporates in each monolayer of growth while the remainder segregates to the new surface. This is consistent with calculations by Neugebauer et al. $^{82}$ that suggest that there are relatively few Ga vacancy surface sites available for $\mathrm{Mg}$ capture during typical growth condition. Orton et al. ${ }^{75}$ used a simple model involving the rate of $\mathrm{Mg}$ capture (chemisorption) from a pool of weakly physisorbed $\mathrm{Mg}$ to show that the fraction of surface sites available for capturing $\mathrm{Mg}$ atoms is $\sim 5 \times 10^{-4}$. This number is quite close to our estimation for a similar growth temperature.

\subsubsection{Surface Rearrangement and Polarity Inversion}

An interesting, and for a time, puzzling SIMS result was obtained for one particular Mg step-doped Ga-polar sample. As is depicted in Figure 4.5, when the $\mathrm{Mg}$ oven was first opened at $400^{\circ} \mathrm{C}$ the $\mathrm{Mg}$ signal at a depth of $\sim 2.5 \mu \mathrm{m}$ from the surface of the sample showed a drastic decrease in concentration. Further incorporation rates were more consistent with $\mathrm{Mg}$-doping in $\mathrm{N}$-polar $\mathrm{GaN}$ even thought this sample was grown on an MOCVD template. The lower Mg-incorporation rate for $\mathrm{N}$-polar material explains this phenomenon when combined with the idea of polarity inversion. Ramachandran et al. $^{79}$ have reported that the presence of about a monolayer of $\mathrm{Mg}$ on the Ga-polarity GaN surface during growth results in the surface inverting to N-polarity. The 


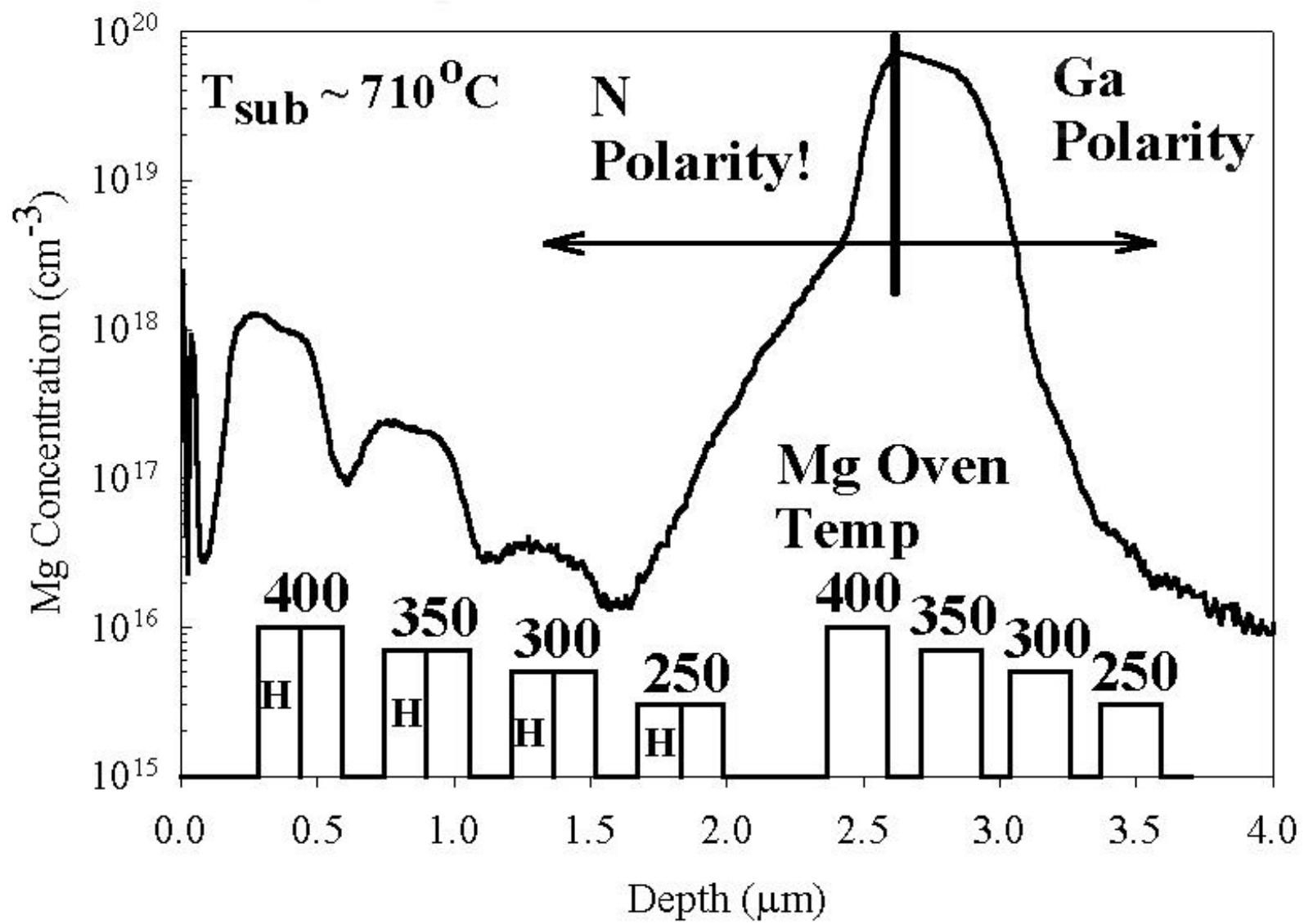

Figure 4.5 SIMS measurement of Mg showing an anomalous decrease in incorporation with high incident $\mathrm{Mg}$ flux. Shown schematically on the bottom of the figure are the opening and closing positions of the $\mathrm{Mg}$ shutter. 
SIMS measurement indicated a dramatic decrease in $\mathrm{Mg}$ incorporation, from $\sim 10^{20}$ to $\sim 10^{18} \mathrm{~cm}^{-3}$, in going from a Mg-oven temperature of 350 to $400{ }^{\circ} \mathrm{C}$, respectively. The latter oven temperature should result in a flux of approximately one monolayer/sec or greater of $\mathrm{Mg}$ arriving at the surface of the growing sample in our system. Figures 4.6 and 4.7 show the subsequent TEM and convergent electron beam diffraction (CBED) characterizations performed by Dr. Linda T. Romano at the Xerox Palo Alto Research Center. These measurements indicated an inversion from Ga- to N-polarity occurred when the growing layer was exposed to this large Mg flux. ${ }^{83}$ That is, the dramatic decrease in $\mathrm{Mg}$ incorporation is due to the change from Ga-polar to N-polar material since incorporation rates for $\mathrm{Mg}$ are lower in $\mathrm{N}$-polar material.

The polarity inversion proceeds by the formation of a multifaceted inversion domain boundary (IDB) ${ }^{83}$ The IDB is faceted predominantly along the $\{0001\}$ and $\{\mathrm{h}, \mathrm{h},-2 \mathrm{~h}, \mathrm{l}\}$ planes, with $\mathrm{l} / \mathrm{h}$ approximately equal to 3 , as seen in the high resolution TEM image of Figure 4.6. First-principles total energy calculations show that the $\{\mathrm{h}, \mathrm{h},-2 \mathrm{~h}, 1\}$ segments of the boundary are stabilized by the incorporation of $\mathrm{Mg}$ in threefold coordinated lattice sites. The formation of facets is found to be energetically favorable under the gallium rich conditions used for this sample.

This study of $\mathrm{Mg}$ in GaN allows for the explanation of one of the more puzzling results in the literature: the decrease in electrical activation for a large $\mathrm{Mg}$ flux on Gapolar material. ${ }^{44,73}$ In these studies, the p-type carrier concentration due to $\mathrm{Mg}$ doping increases with increasing Mg flux up to a certain point. Upon reaching a critical flux of $\mathrm{Mg}$, the electrical activation drops by a significant amount. This can now be understood in terms of surface polarity inversion followed by the reduced $\mathrm{Mg}$ incorporation during the subsequent $\mathrm{N}$-polar growth. When the critical flux of $\mathrm{Mg}$ is reached the surface inverts to N-polarity GaN and the incorporation of $\mathrm{Mg}$ is reduced by more than an order of magnitude. 

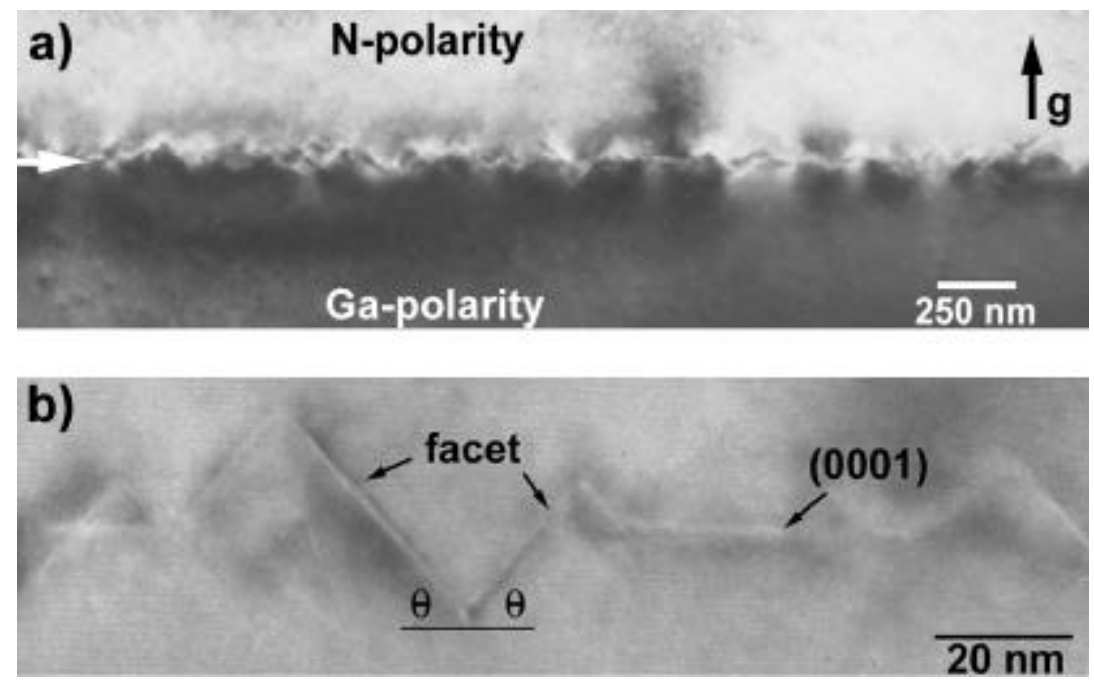

Figure 4.6 (a) High magnification image of the inversion domain boundary (IDB) taken along the [11 20$]$ axis with diffraction vector $g=0002$. (b) Lattice image of the IDB taken along the [1 $\overline{1} 00]$ axis showing facets that form along the (0001) plane and at an angle $\theta \sim 50$ degrees with the basal plane. 

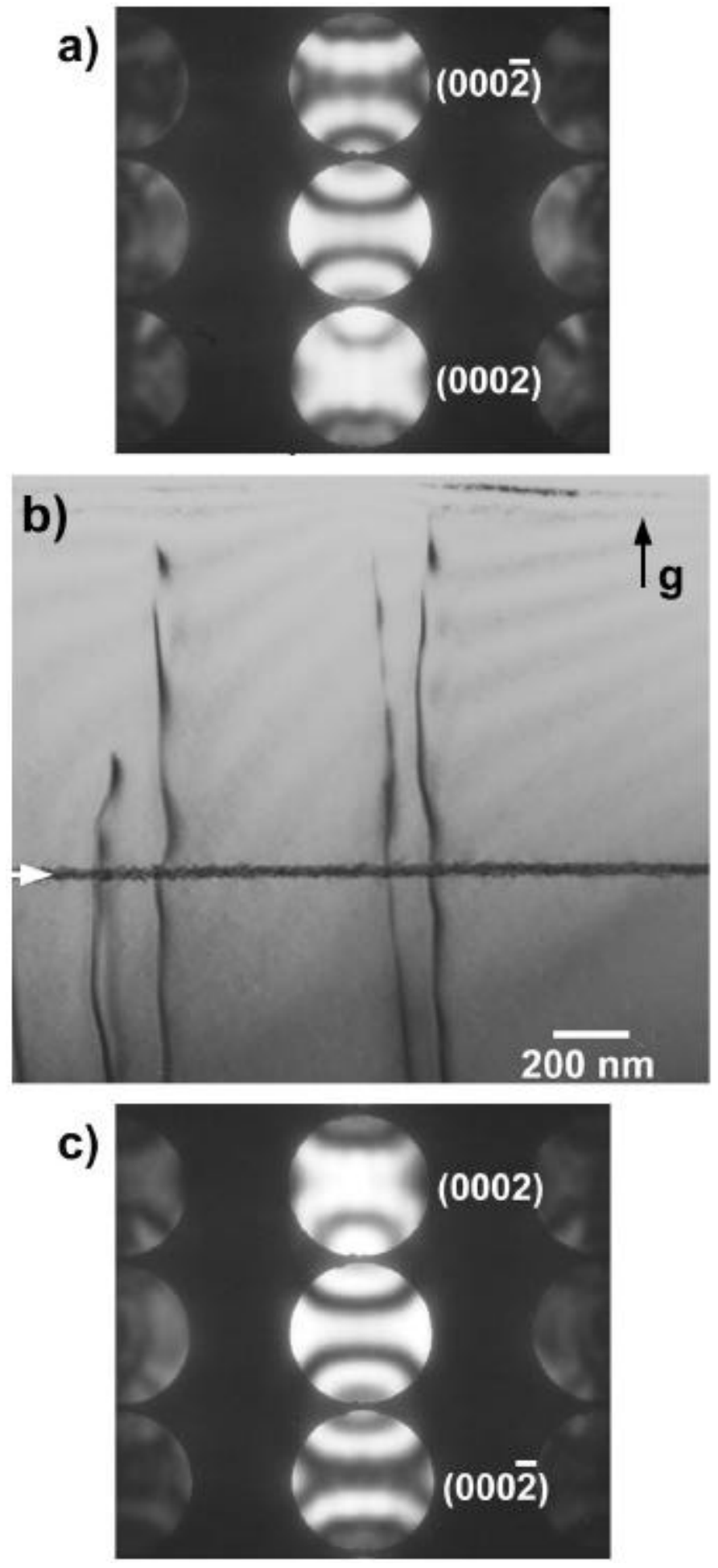

Figure 4.7 Convergent beam electron diffraction patterns taken (a) above and (c) below the interface that is indicated by the arrow in (b). (b) is a bright field image taken with a diffraction vector $\mathrm{g}=0002$ near the $[11 \overline{2} 0]$ axis of a GaN:Mg film. 


\subsection{Optical Characterization}

Power- and temperature-dependent PL measurements have been performed on selected Mg-doped samples. Low temperature reflectance measurements were also used to identify free excitonic transitions in an effort to account for different amount of strain in the samples. Typical features seen in our samples, and in the literature, ${ }^{84,85}$ are an acceptor bond exciton transition $\left(\mathrm{A}^{0}, \mathrm{X}\right)$ at $\sim 3.480 \mathrm{eV}$, a donor-acceptor pair recombination (DAP) located $\sim 3.278 \mathrm{eV}$, and phonon replicas of the DAP and $\left(\mathrm{A}^{0}, \mathrm{X}\right)$ emissions spaced by $\sim 92 \mathrm{meV}$. These features are shown in a low temperature PL scan of a Mg-doped sample in Figure 4.8. The identification of each feature is consistent with power- and temperature-dependent measurements. When assuming a typical donor energy in GaN of $\sim 25-35 \mathrm{meV}$ the DAP peak yields an acceptor energy of $\sim 225 \mathrm{meV}$ for $\mathrm{Mg}$. The localization energy of the $\left(\mathrm{A}^{0}, \mathrm{X}\right)$ peak is $\sim 12 \mathrm{meV}$.

\subsection{Summary}

There is a significant difference in $\mathrm{Mg}$ incorporation depending on polarity with the Ga-polarity incorporating a factor of up to twenty-five times more $\mathrm{Mg}$ than the N-polarity under the Ga-rich growth conditions used. There is also a very strong substrate temperature dependence on $\mathrm{Mg}$ incorporation with more $\mathrm{Mg}$ incorporated at lower temperatures. Consistent with theory, a distinct increase in $\mathrm{Mg}$ incorporation was observed in the presence of atomic hydrogen.

There is strong evidence that a surface-accumulation layer of $\mathrm{Mg}$ is forming during doping. Only a small fraction of the actual $\mathrm{Mg}$ flux is incorporated while the remainder segregates to the new surface. Reports in the literature have shown a decrease in both $\mathrm{Mg}$ incorporation and electrical activation for higher $\mathrm{Mg}$-flux conditions during doping of Ga-polar material. These effects can now be understood in terms of a combination of surface polarity inversion coupled with the lower incorporation rate for N-polarity GaN. 


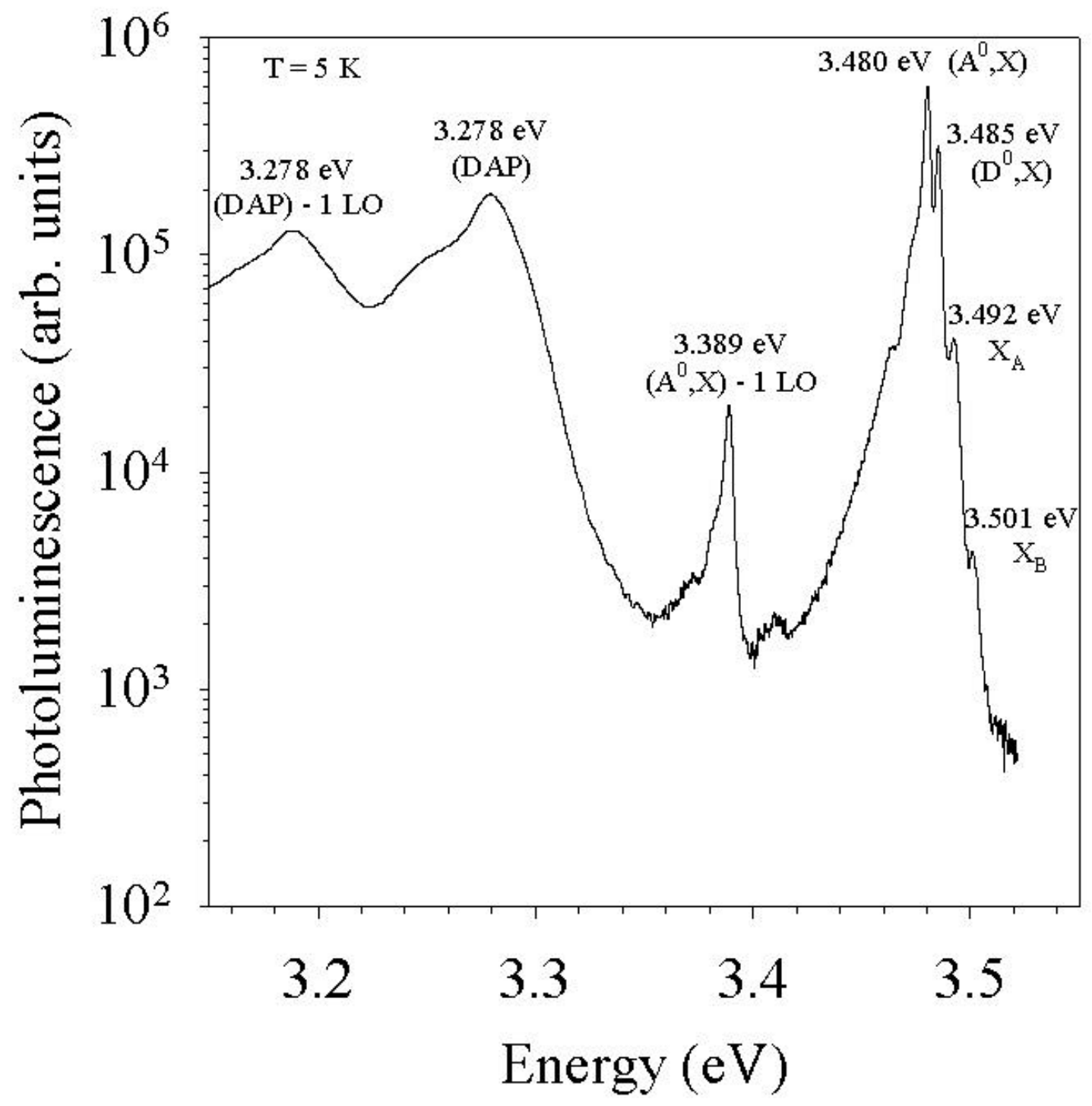

Figure 4.8 Low temperature photoluminescence spectrum of a Mg-doped GaN film. The acceptor bound exciton $\left(\mathrm{A}^{0}, \mathrm{X}\right)$ and donor-acceptor pair (DAP) peaks are prominent. 
For sufficiently high $\mathrm{Mg}$ doping levels the (0001) growth surface of GaN exhibits an instability with respect to polarity inversion, with the formation of a multifaceted IDB. Since $\mathrm{Mg}$ incorporation on the $(000 \overline{1})$ surface is much less efficient than on the $(0001)$ surface, this Mg-induced polarity inversion may limit the p-type doping levels achievable in MBE grown GaN. 


\section{Chapter 5: Beryllium in Gallium Nitride}

As has been illustrated, $\mathrm{Mg}$ is not an ideal impurity for the realization of p-type doping in $\mathrm{GaN}$, displaying many problems that lead to limitations in overall p-type conductivity. There has been a great deal of work done searching for an alternative without much success. Many possible acceptors have been studied, including the Group II elements $\mathrm{Ca}$ and $\mathrm{Zn}$, which have both been shown theoretically and experimentally to form much deeper acceptor levels than $\mathrm{Mg}$, and the Group IV element $\mathrm{C}$. Carbon is amphoteric and hence likes to substitute for both Ga and $\mathrm{N}$ making self-compensation a major drawback. In fact, $\mathrm{C}$ is now often used during the growth of $\mathrm{GaN}$ to produce high resistivity, or semi-insulating, GaN. ${ }^{11}$

Another possibility is the Group II element Be. Be is a common p-type dopant in the more conventional III-V compound semiconductors, such as GaAs. The Be acceptor level was theoretically predicted to be as low as $60 \mathrm{meV}$ above the valence band, ${ }^{86}$ making it potentially a far more shallow acceptor than $\mathrm{Mg}$. While $\mathrm{Mg}$, due to its acceptor level lying at $\sim 200 \mathrm{meV}$, has only about $1 \%$ electrical activity at room temperature, Be with an ionization energy of $60 \mathrm{meV}$ would be $\sim 14 \%$ active. Our own work and the work of other groups indicate that Be forms a level in $\mathrm{GaN}$ with an optical activation energy of $\sim 100 \mathrm{meV}{ }^{87,88,89}$ Hall measurements on Be-doped samples have been less consistent, but reported values fall in a range of $130-170 \mathrm{meV} .{ }^{90,91}$ While neither of these values is as shallow as theoretically predicted, they still represent a potential improvement in electrical activation. This level of activation shows the possibility of a major improvement in p-type conduction in $\mathrm{GaN}$, and is the driving force behind the work that is described in this chapter.

\subsection{Incorporation of Beryllium in GaN}

In order to determine if $\mathrm{Be}$ is a viable acceptor in $\mathrm{GaN}$, it is important to understand how it behaves during growth. To do this, step-doped structures similar to those grown for the study of $\mathrm{Mg}$ incorporation were produced. Ga-polarity samples were 
obtained by growth on MOCVD- or HVPE-grown GaN wafers and N-polarity samples were grown directly on sapphire. The substrates were prepared according to the procedure outlined in Appendix A using the same growth parameters listed for the study of $\mathrm{Mg}$ incorporation. For these samples, the only difference during growth from the $\mathrm{Mg}$ step-doped structures was the use of a 5 cc dopant cell for the evaporation of Be. Again, step-doped structures were produced by opening and closing the Be shutter, and all changes in Be and substrate temperature occurred with the Be shutter closed.

\subsubsection{General Behavior of Beryllium Incorporation}

Be incorporation was studied in the concentration range of $\sim 5 \times 10^{15} \mathrm{~cm}^{-3}$ to $\sim 1 \times 10^{20} \mathrm{~cm}^{-3}$. The incorporation characteristics of Be are slightly different depending on the total concentration, but are in general much better behaved than $\mathrm{Mg}$.

SIMS measurements from the step-doped layers are summarized in Table 5.1. Also shown in the table is the total number of Be atoms incorporated per $\mathrm{cm}^{-2}$ for each step. With the exception of samples 0017 and 0018, these numbers are directly comparable since each step was grown for the same amount of time at identical nitrogen and gallium flux conditions. Be incorporation at higher flux is typically a factor of two larger on N-polarity than Ga-polarity for the same Be flux. Additionally, incorporation is not a strong function of substrate temperature at higher concentrations on either polarity although some temperature dependence is present at low concentrations. This is in stark contrast to the case of $\mathrm{Mg}$ incorporation where significant temperature dependence was observed in all cases. Be incorporation was also studied as a function of III/V ratio. Figure 5.1 illustrates that the incorporation rates for Be do not change significantly even with large changes in the flux ratio. Numerical integration of the peaks indicates that the same number of Be atoms (less than a 5\% increase when going from very Ga-rich growth conditions to $\mathrm{N}$-stable growth) incorporates in each case.

In all, Be appears to be a much better behaved dopant in terms of incorporation than does Mg. Be does not suffer from the drastic substrate temperature or polarity 
Table 5.1 SIMS results for all Be step-doped samples investigated.

\begin{tabular}{|c|c|c|c|c|c|c|c|c|}
\hline Sample & $\begin{array}{l}\mathrm{T}_{\text {sub }} \\
\left({ }^{\circ} \mathrm{C}\right)\end{array}$ & $\begin{array}{l}\mathrm{T}_{\mathrm{Be}} \\
\left({ }^{\circ} \mathrm{C}\right)\end{array}$ & Polarity & $\begin{array}{c}{[\mathrm{Be}]} \\
\left(\mathrm{cm}^{-3}\right)\end{array}$ & $\begin{array}{c}{[\mathrm{Be}]: \mathrm{H}} \\
\left(\mathrm{cm}^{-3}\right)\end{array}$ & $\begin{array}{c}{[\mathrm{Be}] \text { Ratio }} \\
\text { with/ w/out } \\
\mathrm{H}\end{array}$ & $\begin{array}{c}\text { Total Be } \\
\text { incorp. } \\
\left(\mathrm{cm}^{-2}\right)\end{array}$ & $\begin{array}{c}\text { Total Be } \\
\text { incorp. w/H } \\
\quad\left(\mathrm{cm}^{-2}\right)\end{array}$ \\
\hline \multirow{5}{*}{0006} & "675 & "650 & $\overline{\mathrm{N}}$ & $\overline{\mathrm{ND}}$ & $4.5 \times 10^{15}$ & & & $5.4 \times 10^{16}$ \\
\hline & 675 & 700 & $\mathrm{~N}$ & $7.0 \times 10^{15}$ & $8.0 \times 10^{15}$ & 0.9 & $1.2 \times 10^{17}$ & $1.1 \times 10^{17}$ \\
\hline & 675 & 750 & $\mathrm{~N}$ & $6.2 \times 10^{16}$ & -- & & $1.1 \times 10^{17}$ & \\
\hline & 675 & 800 & $\mathrm{~N}$ & $5.0 \times 10^{17}$ & -- & & $8.3 \times 10^{18}$ & \\
\hline & 650 & 750 & $\mathrm{~N}$ & $9.0 \times 10^{16}$ & -- & & $9.0 \times 10^{17}$ & \\
\hline \multirow{5}{*}{0008} & 675 & 650 & $\overline{\mathrm{Ga}}$ & Accum. & $5 \times 10^{14}$ & & & $1.2 \times 10^{16}$ \\
\hline & 675 & 700 & $\mathrm{Ga}$ & Accum. & $2.2 \times 10^{15}$ & & & $9.2 \times 10^{16}$ \\
\hline & 675 & 750 & $\overline{\mathrm{Ga}}$ & Accum. & $5.0 \times 10^{16}$ & & & $8.2 \times 10^{17}$ \\
\hline & 675 & 800 & $\mathrm{Ga}$ & $1.0 \times 10^{17}$ & $5.0 \times 10^{17}$ & & & $7.6 \times 10^{18}$ \\
\hline & 650 & 750 & $\mathrm{Ga}$ & $2.0 \times 10^{17}$ & $1.2 \times 10^{17}$ & 0.6 & $1.8 \times 10^{18}$ & $1.5 \times 10^{18}$ \\
\hline \multirow{3}{*}{0015} & 675 & 800 & $\mathrm{Ga}$ & $1.0 \times 10^{17}$ & -- & & $9.4 \times 10^{18}$ & \\
\hline & 675 & 900 & $\mathrm{Ga}$ & $2.9 \times 10^{18}$ & -- & & $2.3 \times 10^{20}$ & \\
\hline & 675 & 1000 & $\mathrm{Ga}$ & $3.7 \times 10^{19}$ & $2.8 \times 10^{19}$ & 0.9 & $3.0 \times 10^{21}$ & $2.8 \times 10^{21}$ \\
\hline \multirow{3}{*}{0016} & 675 & 800 & $\mathrm{~N}$ & $6.0 \times 10^{16}$ & -- & & $7.2 \times 10^{18}$ & \\
\hline & 675 & 900 & $\mathrm{~N}$ & $2.5 \times 10^{18}$ & -- & & $2.1 \times 10^{20}$ & \\
\hline & 675 & 1000 & $\mathrm{~N}$ & $1.1 \times 10^{20}$ & $5.0 \times 10^{19}$ & 0.5 & $7.3 \times 10^{21}$ & $3.9 \times 10^{21}$ \\
\hline 0017 & 550 & 900 & $\overline{\mathrm{Ga}}$ & $1.9 \times 10^{19}$ & $\overline{--}$ & & & \\
\hline 0018 & 675 & 900 & $\mathrm{Ga}$ & $1.9 \times 10^{19}$ & -- & & & \\
\hline
\end{tabular}




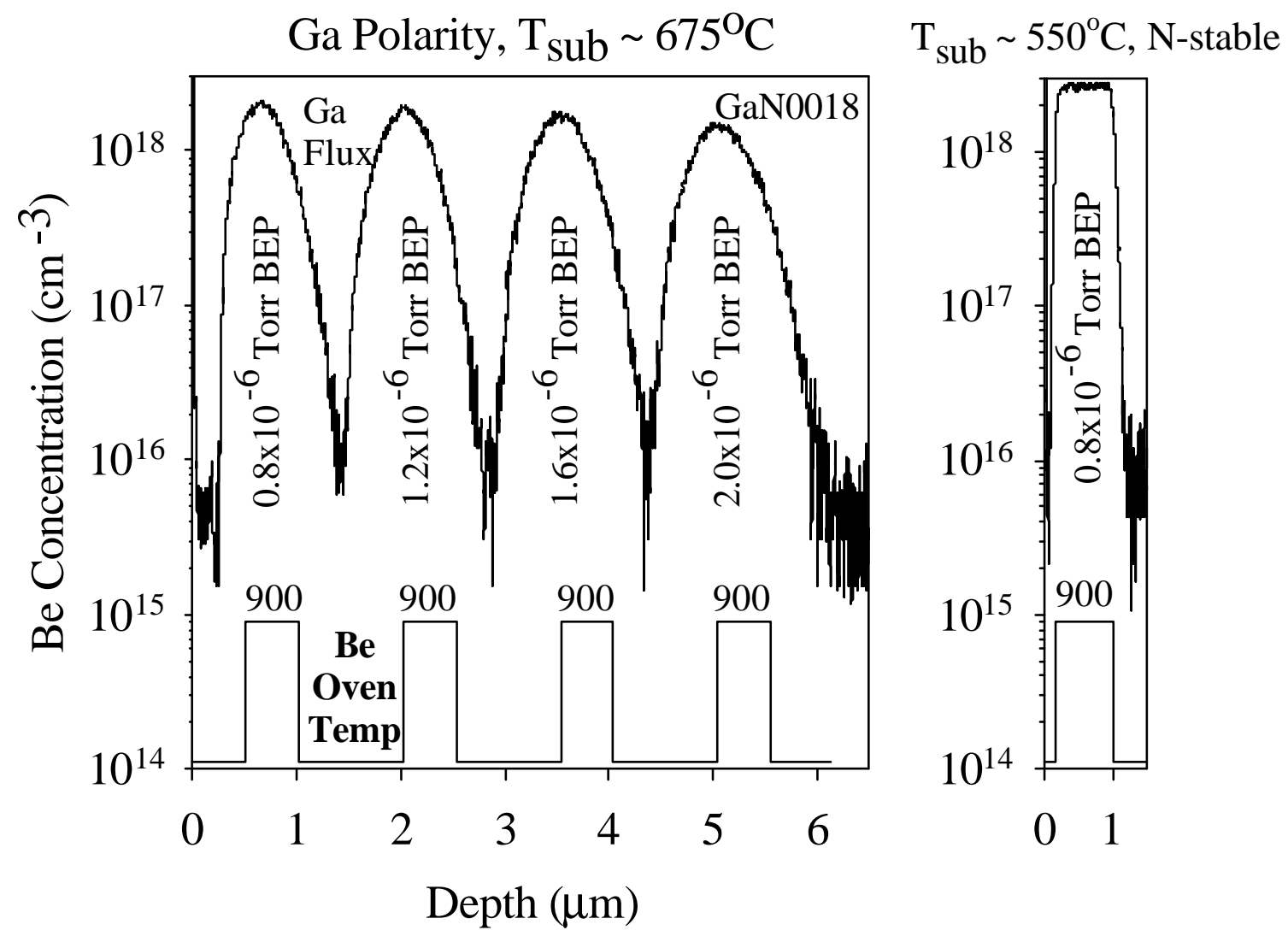

Figure 5.1 SIMS measurement showing the lack of Be incorporation dependence on the growth conditions of GaN. The shutter openings and closings are shown schematically on the bottom of the figures along with the Be oven temperature and Ga fluxes used. 
dependence that is seen during $\mathrm{Mg}$ doping. If Be can be used as a p-type dopant in $\mathrm{GaN}$, this lack of dependence on growth conditions would be preferable to doping with $\mathrm{Mg}$ since less stringent growth requirements would need to be met. This "ease of use" can have a sizeable effect on material yield.

\subsubsection{Effects of Atomic Hydrogen}

Many of the step-doped layers were also used to investigate growth under atomic hydrogen. The addition of hydrogen is theorized to enhance the incorporation of $\mathrm{Be}$ in $\mathrm{GaN}$ as it does for $\mathrm{Mg} .{ }^{92}$ Similar to the $\mathrm{Mg}$ case for Ga-polar growth, the presence of atomic hydrogen enhances incorporation at low fluxes and sharpens the profiles at higher fluxes. In contrast to the calculations, however, atomic hydrogen did not seem to influence Be incorporation at higher Be fluxes or for any $\mathrm{N}$-polarity samples investigated.

SIMS measurements made on the Be-doped samples indicated a low background for both oxygen and carbon, less than the background (low-to-mid $10^{16} \mathrm{~cm}^{-3}$ ) for these measurements. Measurements also indicate that hydrogen is incorporated at levels at or above the Be concentration when grown under atomic $\mathrm{H}$, in contrast to the incorporation of $\mathrm{Mg}$. This implies that Be-H complexes may be forming, providing an additional compensation mechanism for doped material. The Be-H formation energy has been calculated to be higher than the corresponding $\mathrm{Mg}-\mathrm{H}$ bond energy. ${ }^{92}$ The current results indicate that while $\mathrm{Mg}-\mathrm{H}$ bonds may be broken at the temperatures used during $\mathrm{MBE}$ growth allowing the compensating $\mathrm{H}$ to anneal out, the $\mathrm{Be}-\mathrm{H}$ bonds remain stable. An increase in the background oxygen and carbon was also observed for growth under atomic $\mathrm{H}$.

\subsection{Surface Accumulation/Segregation}

\subsubsection{Evidence for Surface Accumulation/Segregation}

As seen in Chapter 4, surface accumulation/segregation effects play a large role in the incorporation kinetics of $\mathrm{Mg}$ in $\mathrm{GaN}$. Be also shows signs of surface accumulation, although the process may be different for the two dopants. $\mathrm{Mg}$ exists as a stable configuration of atoms on the surface of the growing $\mathrm{GaN}$ while a small fraction 
incorporates during each layer of growth. Theoretical results have not indicated that Be has such a stable configuration. ${ }^{93}$ Although the mechanisms for incorporation of $\mathrm{Mg}$ and Be are apparently different, the results are similar in many respects. As indicated in Figure 5.2 for a Ga-polar sample, at low concentrations the Be incorporation profile appears to be that expected for classic surface accumulation/segregation. There are no steps apparent in the trace, even though the Be flux was interrupted several times. While diffusion effects are undoubtedly present as well, this pronounced lack of any step-like structure is consistent with surface accumulation. Also, the steps of the Be profile seen in Figure 5.3 representing high doping concentrations were offset with respect to the opening and closing of the dopant shutter, also consistent with surface accumulation. It is important to know how much $\mathrm{Be}$ is residing on the surface during growth. This amount can be estimated for these samples due to a somewhat fortuitous effect of the RHEED beam.

On many N-polarity samples we observed evidence of a stripe associated with the high-energy electron irradiation occurring during RHEED measurements. Effects of electron irradiation from the RHEED beam during MBE growth have been previously reported. ${ }^{94,95,96}$ Of interest to the topic at hand, Figure 5.4 shows a comparison between the measured Be concentration profiles inside and just outside such a RHEED stripe. It appears that in this case electron irradiation suppresses surface accumulation of Be. The same number of atoms is incorporated in each case but the irradiated section lines up much more closely with the opening and closing of the Be shutter. Thus, the suppression of surface accumulation under electron irradiation allows an estimate of the Be diffusion coefficient to be made for this temperature $\left(700^{\circ} \mathrm{C}\right)$ of $1 \times 10^{-15} \mathrm{~cm}^{2} \mathrm{~s}^{-1}$. Knowing the diffusion coefficient, an estimate of the number of Be atoms on the sample surface can be obtained with some confidence by counting the Be incorporated after the doping shutter is closed. From the SIMS measurement shown in Figure 5.3, summing results in $3 \times 10^{12}$, $7 \times 10^{13}$ and $8 \times 10^{14} \mathrm{Be}$ atoms $/ \mathrm{cm}^{2}$ on the GaN surface for Be oven temperatures of 800 , 900 and $1000^{\circ} \mathrm{C}$, respectively. For equivalent doping in N-polarity GaN, surface concentrations of $2 \times 10^{12}, 5 \times 10^{13}$ and $7 \times 10^{14} \mathrm{Be}$ atoms $/ \mathrm{cm}^{2}$ were observed for the same sequence of Be oven temperatures. The results of the SIMS measurements indicate that 


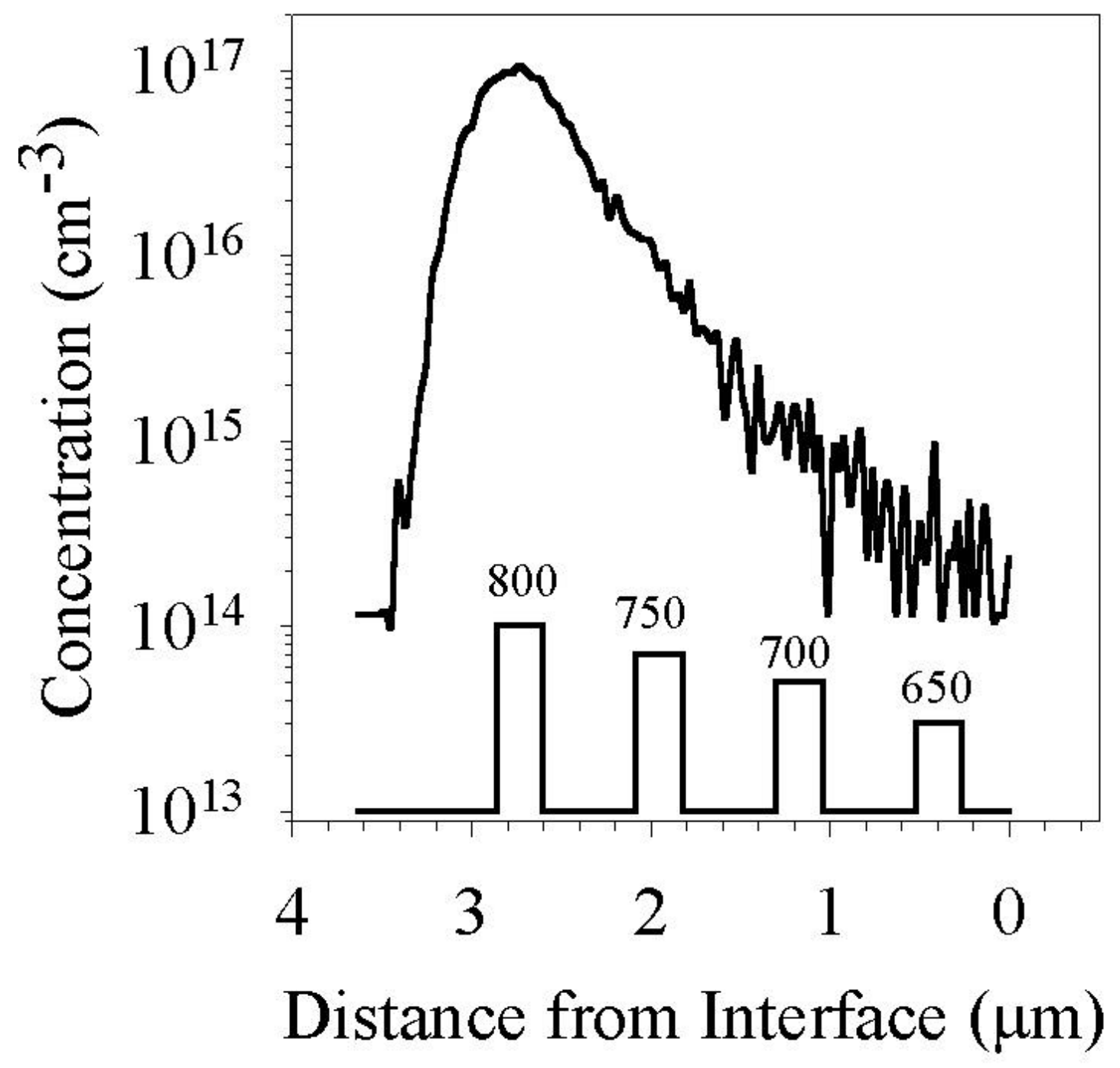

Figure 5.2 SIMS measurement of the Be concentration in a Ga-polar GaN sample.

The lack of any discernable step-like structure is indicative of surface accumulation. 


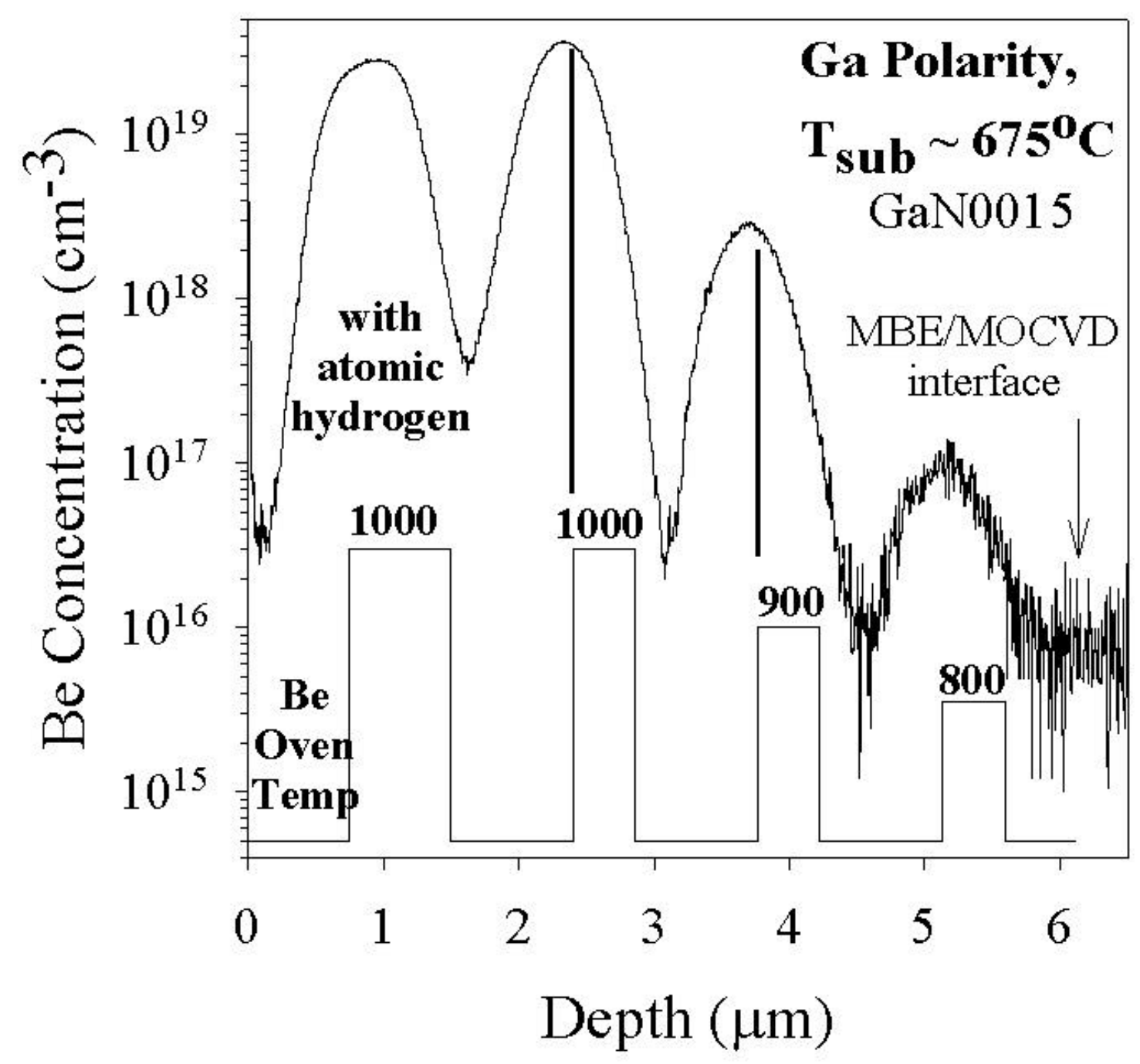

Figure 5.3 SIMS measurement showing the Be incorporated into a Ga-polar sample. Shown schematically on the bottom of the figure are the opening and closing positions of the Be shutter, as well as the Be furnace temperatures. 


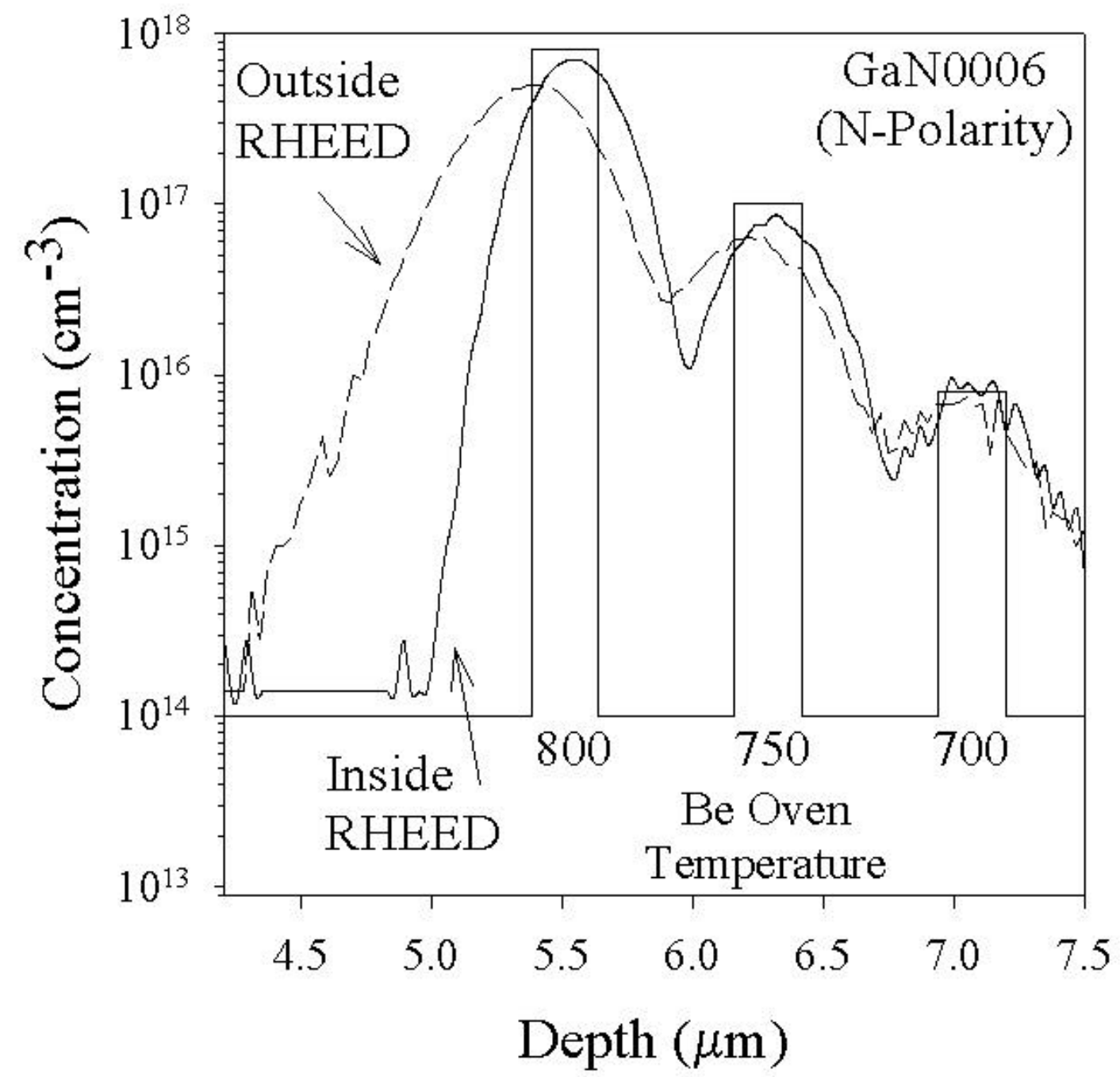

Figure 5.4 SIMS measurement inside and just outside the stripe created by the electron irradiation of the RHEED beam. The high-energy electrons appear to suppress the formation of the accumulation layer. Shown schematically on the bottom of the figure are the opening and closing positions of the Be shutter, as well as the Be furnace temperatures. 
for the largest surface accumulation essentially a monolayer (approximately $1.1 \times 10^{15}$ atoms $/ \mathrm{cm}^{2}$ ) of Be resides on the surface during growth.

In addition to the Be-profile shapes, most of the SIMS measurements were also suggestive of a higher concentration of $\mathrm{Be}$ at the surface similar to $\mathrm{Mg}$ doped samples. Again, these high concentration data points persisted deeper into the sample than can be easily explained by measurement-related effects. Auger measurements were made on several step-doped and uniformly doped layers using the same equipment as that employed for nitridation studies. Auger measurements have a very small probability of detecting electron emission from Be, so the fact that a Be peak was observed at all is an indication of significant Be surface coverage. Although quantitative interpretation was complicated due to the presence of a Ga satellite peak located near the energy of the $\mathrm{Be}$ Auger transition, the presence of Be was measured to be at levels representing a significant fraction of a monolayer. The Auger and near-surface SIMS measurements are consistent with the numbers reported above for incorporation after the Be shutter was closed. As further evidence of the segregation effect, it should be noted that the step-doped samples were capped with approximately $0.25 \mu \mathrm{m}$ of undoped material, yet still showed a significant contribution from Be in the Auger scans. This surface layer of Be metal may have a significant influence on the as-grown properties, as will be seen with the electrical characterization of these doped samples.

\subsubsection{Structural Effects of Beryllium Incorporation}

Dr. L.T. Romano performed TEM at the Xerox Palo Alto Research Center on several of the step-doped layers. Figure 5.5 is a TEM micrograph of the step-doped sample with the SIMS profile shown in Figure 5.3. The MBE layer shown was grown on an MOCVD template and is hence Ga-polar. As is typical with appropriate cleaning and growth initiation, there was no structural evidence of an interface between the MOCVD and MBE material. There are threading dislocations visible in Figure 5.5 that proceed without interruption into the MBE layer that are typically stable for growth along the caxis. The dashed lines in the figure represent the MBE/MOCVD interface and the first 


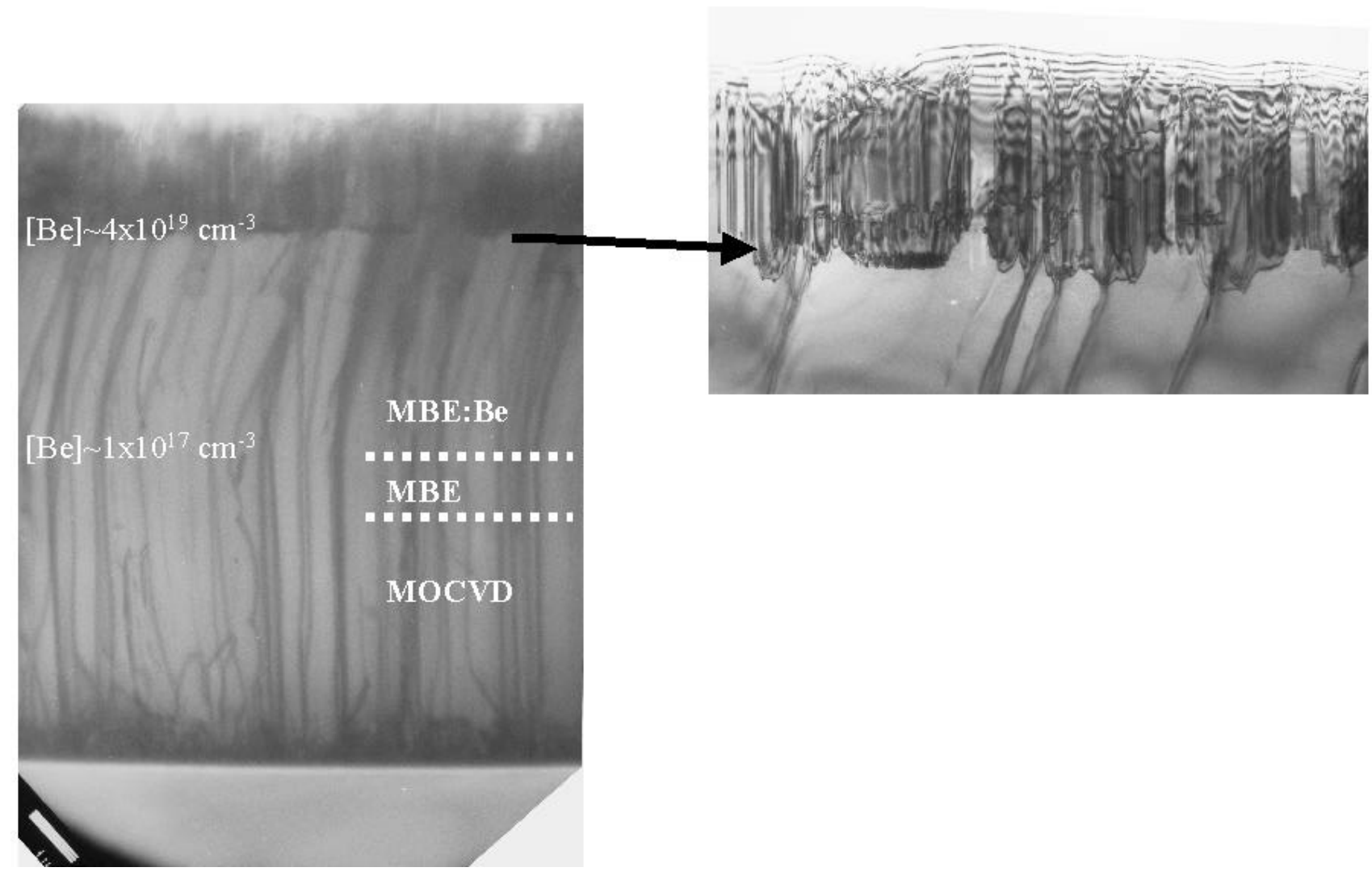

Figure 5.5 TEM micrographs of the same sample as shown in Figure 5.3. The dislocations bend (left image) upon the first introduction of Be, while high concentrations of Be promote polarity inversion and defect formation (right image). 
introduction of $\mathrm{Be}$ (at a level of about $10^{17} \mathrm{~cm}^{-3}$ ) in the sample. Two important features are observed in the TEM analysis. First, when Be is first introduced into the growing MBE layer (the upper dashed line) the dislocations are seen to bend at an angle of about $20^{\circ}$. The "bent" dislocations then continue to propagate at this angle in a stable fashion, without any observable effect either due to the closing of the Be shutter which results in a decreasing Be concentration or the next opening of the shutter which results in Be incorporation at levels up to the low $10^{18} \mathrm{~cm}^{-3}$ level. This effect was unexpected, as there have been no reports of bending dislocations for any other uniform GaN growth. The behavior of these dislocations suggests that the surface may experience considerable strain during growth due to Be-doping.

The second and most significant observation, however, is the extreme degradation in structural quality that occurs in this sample when Be was incorporated at levels of about $4-5 \times 10^{19} \mathrm{~cm}^{-3}$, corresponding to a Be oven temperature of $1000^{\circ} \mathrm{C}$. The primary mechanism appeared to be related to the formation of inversion domains similar to what has been observed in many cases of heavy Mg doping by both MOCVD and MBE. ${ }^{79,97}$ Similar observations were made for N-polar samples, grown directly on sapphire by MBE. In stark contrast to the case of Mg-induced polarity inversion, however, this inversion is not as "clean". As seen in the high resolution TEM images in Figures 5.5 and 5.6, there is a profound degradation in sample quality by the formation of inversion domains and cubic grains at the interface.

Since Raman spectroscopy can be used to assess strain in $\mathrm{GaN}^{29,30}$ Raman measurements were made on several uniformly Be-doped layers in Prof. Nancy C. Giles' laboratory. Typically, the structures measured consisted of a $1-\mu \mathrm{m}$ thick Be-doped layer grown on a 2- $\mu \mathrm{m}$ thick undoped MOCVD layer, and thus measured Raman shifts were indicative of strain in the composite system. Be levels of $10^{18} \mathrm{~cm}^{-3}$ or less indicated little strain, while levels near the onset of degradation $\left(\sim 4 \times 10^{19} \mathrm{~cm}^{-3}\right)$ indicated a significant compressive strain for the composite system. 


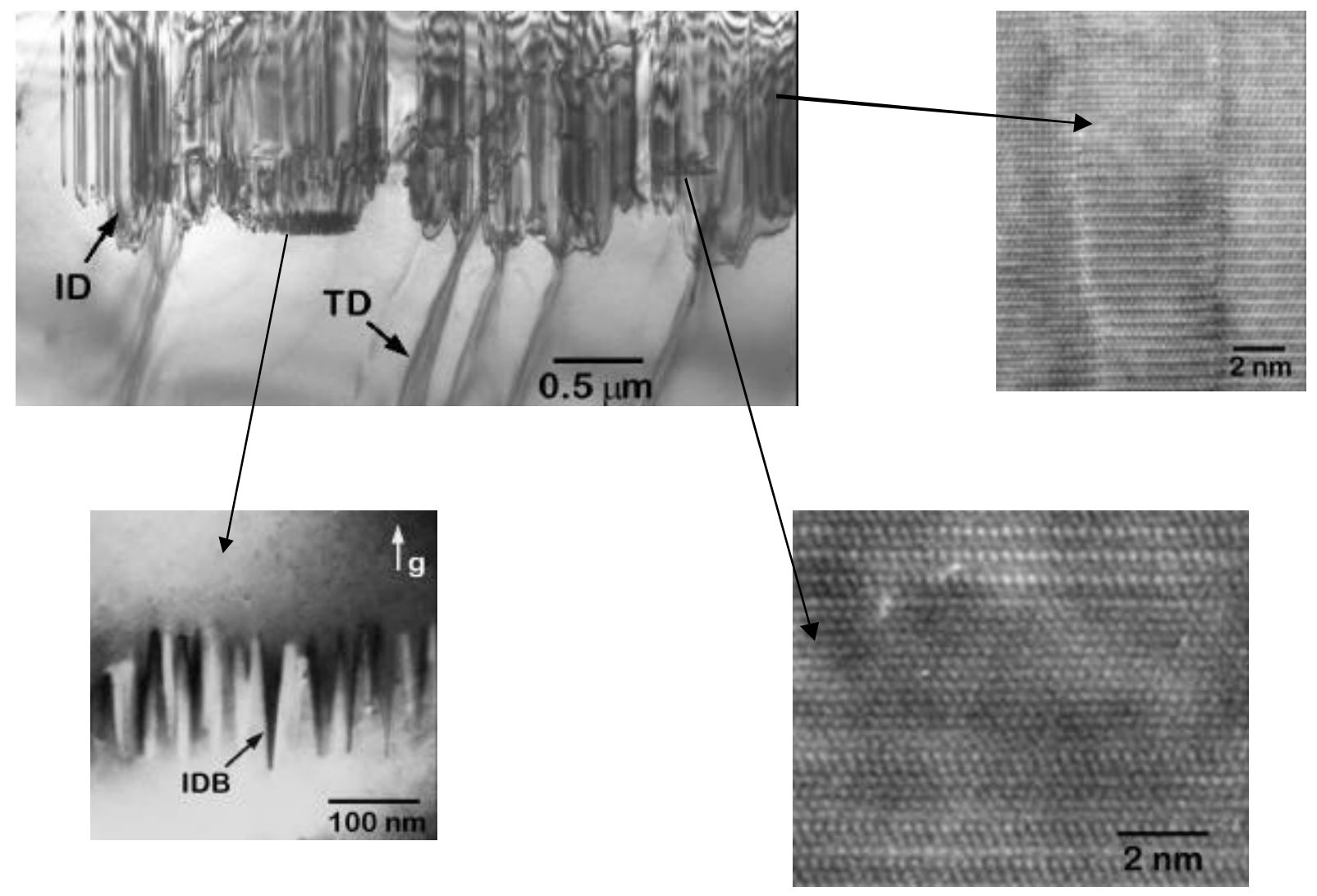

Figure 5.6 High resolution TEM micrographs of the sample shown in Figure 5.5. The lower left and upper right images show inversion domains and the image at the lower right shows a cubic grain. 
The Raman result coupled with surface accumulation suggests a mechanism for the observed effects of Be incorporation on structural properties. The measured Be surface concentration would correspond to a very large compressive strain at the surface of the growing layer. This strain could promote a slight tilting of the growth front, resulting in the "bent" dislocations in Figure 5.5. At the highest doping levels, surface concentrations of Be approach a complete monolayer, resulting in enough strain to cause surface inversion and dislocation formation to occur in an attempt to relieve the strain. Of course, for near-monolayer coverage, the Be concentration may be so large that solubility or second phase formation may be interfering with the growth. Regardless of the mechanism, it is clear that surface accumulation of Be under standard GaN growth conditions by MBE ultimately leads to a breakdown of structural quality, and sets a limit on the maximum Be concentration attainable. It is of interest that the phenomenon of structural degradation was observed for Be doping of cubic $\mathrm{GaN}$ at similar incorporation levels, $\sim 10^{20} \mathrm{~cm}^{-3} .90$

\subsection{Optical Characterization}

Figures 5.7a and 5.7b show low-temperature photoluminescence spectra from two of the Be-doped GaN layers. All N-polar Be-doped GaN exhibited a strong feature near $3.38 \mathrm{eV}$, which is the most prominent feature for most samples as shown in Figure 5.7. Temperature and power dependence studies consistently indicate that the $3.38 \mathrm{eV}$ peak is donor-acceptor pair luminescence. A previous study of Be-doped GaN has also reported the same feature with a similar interpretation. ${ }^{98}$ The only other features are phonon replicas of donor-acceptor pair luminescence and a bound exciton at $\sim 3.475 \mathrm{eV}$. Using the typical shallow donor energy in $\mathrm{GaN}$ of 20 to $35 \mathrm{meV}$, this indicates an acceptor ionization level at about $100 \mathrm{meV}$. The previously unreported feature at $3.397 \mathrm{eV}$ shown in Figure 5.7a was observed on several of the p-type samples, and has a temperature and power dependence characteristic of an electron-acceptor transition. The transition lineshape obtained using weak below-gap illumination was consistent with this interpretation. Again, this would indicate an acceptor level $\sim 100$ to $110 \mathrm{meV}$ above the valence band. The feature at $3.472 \mathrm{eV}$ in Figure $5.7 \mathrm{a}$ is tentatively assigned to an acceptor-bound exciton with a binding energy of 9 to $10 \mathrm{meV}$. This binding energy is 


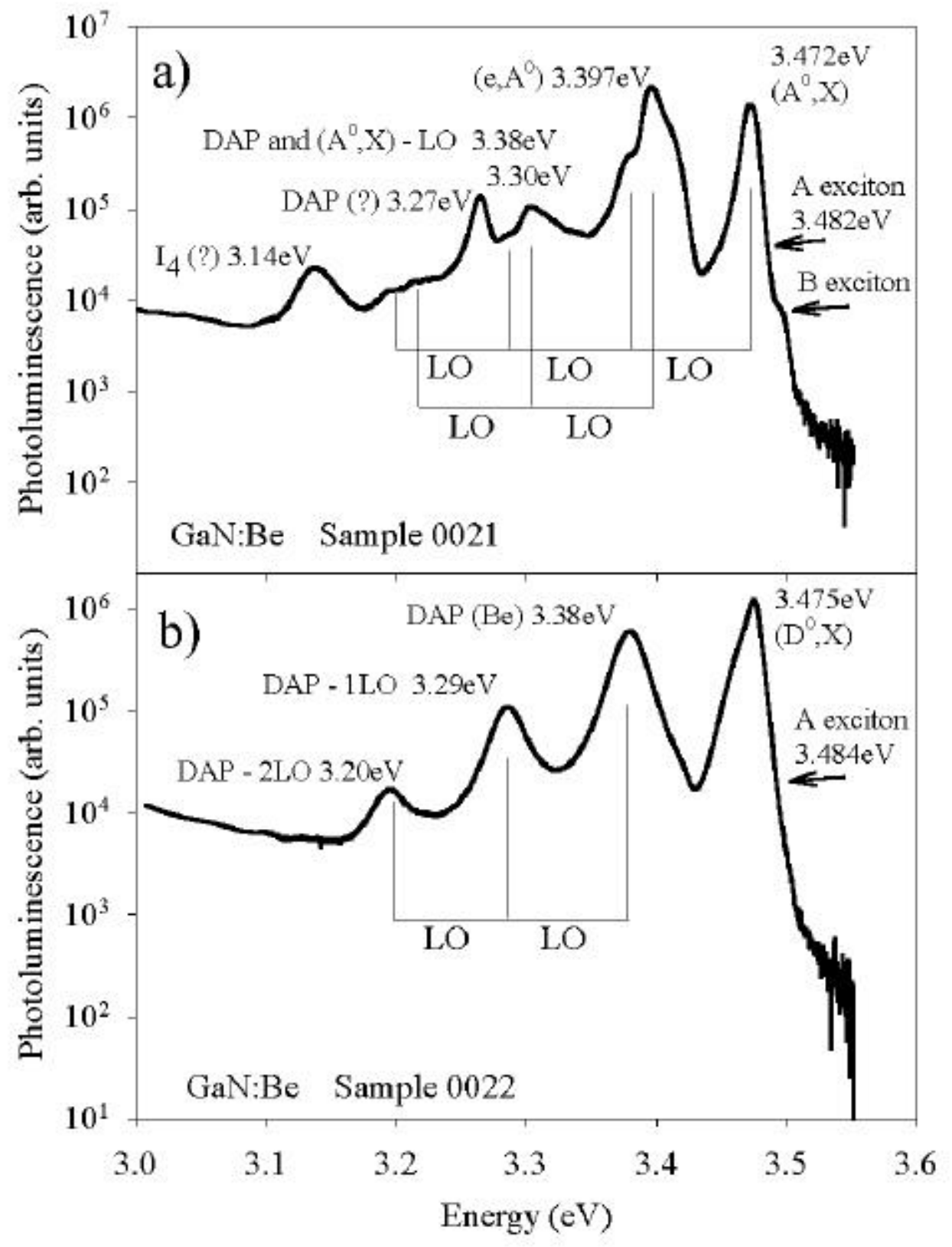

Figure 5.7 Low temperature photoluminescence measurements of Be-doped GaN. Both samples show evidence of a donor-acceptor pair (DAP)at an energy of $3.38 \mathrm{eV}$, while the sample in a) shows the electron-acceptor $\left(e, A^{0}\right)$ transition. 
again consistent with Be having a smaller ionization energy than $\mathrm{Mg}$, which has an exciton binding energy of $12.6 \mathrm{meV}$.

The feature at $3.27 \mathrm{eV}$ in Figure 5.7a has also been previously reported for Bedoped material and has donor-acceptor pair characteristics. ${ }^{99}$ There is a transition near $3.14 \mathrm{eV}$ that has previously been labeled the $\mathrm{I}_{4}$ transition. Interestingly, these latter two features were removed after etching the surface of the GaN (see Section 5.4), indicating they may be related to the surface accumulation layer.

\subsection{Electrical Characterization}

Electrical measurements on Be-doped GaN samples were complicated in several respects. First, in the case of Ga-polar growths, all of the early samples were grown on n-type conducting substrates. This complicates the Hall measurements as the measured values are for the composite doped-layer/substrate system. Any p-type conductivity that was present in the samples may not have been high enough for the composite system to appear p-type, as the n-type background in many of these substrates was in the $10^{17}$ to $10^{18} \mathrm{~cm}^{-3}$ range. Ideally, a p-type layer grown on an n-type substrate should form a p-n junction that will create a depletion region and confine the electrical measurements to the p-type layer. In the case of $\mathrm{GaN}$, however, the current can shunt along dislocations that propagate parallel to the $\mathrm{c}$-axis in the crystal to the underlying n-type layer, defeating the junction isolation. Later Ga-polar samples were grown on semi-insulating MOCVD wafers to combat this problem.

Perhaps most importantly, there is the matter of the Be surface accumulation layer and its effects on the electrical measurements. It is probable that a residual layer of $\mathrm{Be}$ metal resides on the surface of the as-grown sample, as has been witnessed through SIMS and Auger measurements. This metallic layer would serve as a short between contacts, crippling any possibility of useful Hall results. Consequently, many of our early measurements performed on Be-doped material were difficult to interpret. High background voltages and spurious readings were common. Acid etching using aqua regia (3:1 $\left.\mathrm{HCl}: \mathrm{HNO}_{3}\right)$ was attempted to remove the surface Be with limited success. This 
process proved non-optimal as the etched surfaces were quite rough. Table 5.2 lists the growth conditions and electrical measurements, where possible, of the Be-doped samples. (a) C-doped MBE-grown substrates. Contact issues and high background voltages are typically responsible when results are listed as "not measurable."

Of the samples where Hall measurements were possible, five different N-polar samples exhibited p-type conduction. All five samples were doped with $1-3 \times 10^{18} \mathrm{~cm}^{-3}$ Be atoms but the highest carrier concentration measured was $\sim 8 \times 10^{16} \mathrm{~cm}^{-3}$. This would indicate that most of the $\mathrm{Be}$ is compensated. One possibility for the compensation mechanism is the formation of Be interstitials that would act as donors in the system. Be interstitials have been predicted to have a low formation energy in $\mathrm{GaN}$ and may be present at high concentrations. ${ }^{92}$ Additionally, Be interstitials are predicted to have a low barrier to diffusion and are coulombically attracted to the substitutional Be acceptors indicating that compensated pairs could easily form. To date, Be interstitials have not been found experimentally but their possible effects on the electrical properties of $\mathrm{GaN}$ cannot be ignored. Interestingly, the measured hole mobilities of these four samples are quite high, with a range of $\sim 20-200 \mathrm{~cm}^{2} / \mathrm{V}$-sec, significantly higher than those found in $\mathrm{Mg}$-doped material which range from $1-10 \mathrm{~cm}^{2} / \mathrm{V}$-sec. It is possible that the increased mobilities are due to the lower scattering cross-sections that would result from the coulombically attracted substitutional and interstitial Be pairs. Single coulomb scatterers have a higher scattering cross-section and would limit carrier mobilities.

The last four samples listed in Table 5.2 were all grown on semi-insulating MOCVD substrates from the Naval Research Laboratory in an attempt to eliminate the effect of substrate conduction during Hall measurements. In each case, measurements were not possible due to the high resistivity of the samples (no current flow was detectable with a maximum input voltage of $12 \mathrm{~V}$ ).

To date only one Ga-polar sample has been measured as ptype, and only one sample has shown any temperature dependence during Hall measurements. This sample, GaN0018, was a step-doped structure used to calibrate the incorporation of Be vs Ga 
Table 5.2 Electrical results from Be-doped GaN.

\begin{tabular}{|c|c|c|c|c|c|c|c|}
\hline Sample & Substrate & $\begin{array}{c}\text { Substrate } \\
\text { Dial Temp } \\
\left({ }^{\circ} \mathrm{C}\right)\end{array}$ & $\begin{array}{c}\text { Be Temp } \\
\left({ }^{\circ} \mathrm{C}\right)\end{array}$ & $\begin{array}{c}\text { Ga Flux } \\
\left(\mathrm{x} 10^{-7} \text { Torr }\right)\end{array}$ & $\begin{array}{c}\text { Growth Rate } \\
(\mu \mathrm{m} / \mathrm{hr})\end{array}$ & $\begin{array}{l}\text { RT Hall Density } \\
\qquad\left(\mathrm{cm}^{-3}\right)\end{array}$ & $\begin{array}{l}\text { RT Mobility } \\
\left(\mathrm{cm}^{2} / \mathrm{V}-\mathrm{sec}\right)\end{array}$ \\
\hline 0010 & MOCVD & 675 & 750 & 15 & 0.5 & $-8.7 \times 10^{17}$ & 475 \\
\hline 0018 & MOCVD & 675 & 900 & 16 & 0.5 & $1.7 \times 10^{17}$ & 12 \\
\hline 0019 & sapphire & 675 & 900 & 16 & 0.5 & not measurable & not measurable \\
\hline 0020 & sapphire & 675 & 900 & 16 & 0.5 & not measurable & not measurable \\
\hline 0021 & sapphire & 675 & 900 & 9 & 0.5 & $5.5 \times 10^{15}$ & 170 \\
\hline 0022 & sapphire & 675 & 900 & 16 & 0.5 & $1.7 \times 10^{15}$ & 207 \\
\hline 0025 & $\mathrm{MBE}^{(\mathrm{a})}$ & 675 & 900 & 16 & 0.5 & $7.5 \times 10^{16}$ & 83 \\
\hline 0026 & $\mathrm{MBE}^{(\mathrm{a})}$ & 675 & 900 & 29 & 0.25 & $1.1 \times 10^{16}$ & 31 \\
\hline 0028 & $\mathrm{MBE}^{(\mathrm{a})}$ & 700 & 950 & 13 & 0.25 & $7.5 \times 10^{15}$ & 23 \\
\hline 0031 & sapphire & 700 & 950 & 10 & 0.25 & not measurable & not measurable \\
\hline 0032 & sapphire & 700 & 950 & 8 & 0.25 & $-7.5 \times 10^{14}$ & 11 \\
\hline 0033 & sapphire & 675 & 950 & 8 & 0.25 & $-1.4 \times 10^{13}$ & 558 \\
\hline 0035 & sapphire & 675 & 950 & 16 & 0.25 & not measurable & not measurable \\
\hline 0038 & sapphire & 675 & 900 & 9 & 0.5 & not measurable & not measurable \\
\hline 0039 & sapphire & 675 & 900 & 9 & 0.5 & not measurable & not measurable \\
\hline 0040 & sapphire & 675 & 900 & 9 & 0.5 & not measurable & not measurable \\
\hline 0072 & MOCVD & 675 & 792 & 16 & 0.25 & $\sim-3 \times 10^{16}$ & 60 \\
\hline 0073 & MOCVD & 675 & 855 & 16 & 0.25 & $\sim-5 \times 10^{15}$ & 200 \\
\hline 0074 & MOCVD & 675 & 750 & 16 & 0.25 & semi-insulating & semi-insulating \\
\hline 0083 & MOCVD & 675 & 805 & 12 & 0.25 & semi-insulating & semi-insulating \\
\hline 0084 & MOCVD & 675 & 805 & 9 & 0.25 & not measurable & not measurable \\
\hline 0085 & MOCVD & 675 & 805 & 9 & 0.25 & not measurable & not measurable \\
\hline 0086 & MOCVD & 675 & 805 & 7 & 0.25 & not measurable & not measurable \\
\hline 0087 & MOCVD & 675 & 805 & 2 & 0.25 & $-5.9 \times 10^{17}$ & 75 \\
\hline 0095 & MOCVD & 675 & 805 & 8 & 0.25 & semi-insulating & semi-insulating \\
\hline 0097 & MOCVD & 675 & 805 & 4 & 0.25 & semi-insulating & semi-insulating \\
\hline 00128 & MOCVD & 780dial & 925 & 10 & 0.17 & semi-insulating & semi-insulating \\
\hline 0101 & MOCVD & 780dial & 840 & 17 & 0.17 & semi-insulating & semi-insulating \\
\hline
\end{tabular}


flux. Approximately the top $2 \mu \mathrm{m}$ of the sample were etched away using chemically-assisted ion-beam etching (CAIBE) to eliminate any effect of the surface accumulation layer. The temperature-dependent Hall mobility and carrier concentration data shown in Figure 5.8 display a wide amount of scatter indicating continuing problems with electrical measurements. These problems are seen not only for Be doping, but for $\mathrm{Mg}$ doping as well, indicating that they may be due to difficulties in characterizing p-type $\mathrm{GaN}$ in general. Results such as these have led some groups to use much smaller areas of samples for Hall measurements, typically on the order of $50-100 \mu \mathrm{m}$ on a side. Measurements are then performed in a number of places on the sample in the hope of finding a region that behaves well electrically.

The conductivity of the sample is typically a much more robust number generated by these measurements. The temperature dependent conductivity of this sample is shown in Figure 5.9. The conductivity, $\sigma$, depends on the mobility and the carrier concentration:

$$
\sigma=p e \mu
$$

Both the mobility, $\mu$, and the carrier concentration, $\mathrm{p}$, depend on temperature. The carrier concentration has an additional dependence on temperature of $\mathrm{T}^{3 / 2}$ due to the near-gap density of states as well as an exponential temperature dependence:

$$
p(T)=\frac{1}{4}\left(\frac{2 m_{v}^{*} k_{B} T}{\pi \hbar^{2}}\right)^{\frac{3}{2}} e^{\frac{-E_{a}}{k_{B} T}}
$$

It is difficult, therefore, to extract an activation energy from the conductivity. However, scattering, and hence mobility, is typically assumed to be due to acoustic phonons at higher temperatures. This scattering mechanism has a temperature dependence of $\mathrm{T}^{3 / 2}$. Since this effectively cancels the density of states dependence, the only dependence on temperature left in the conductivity comes from the exponential. In 


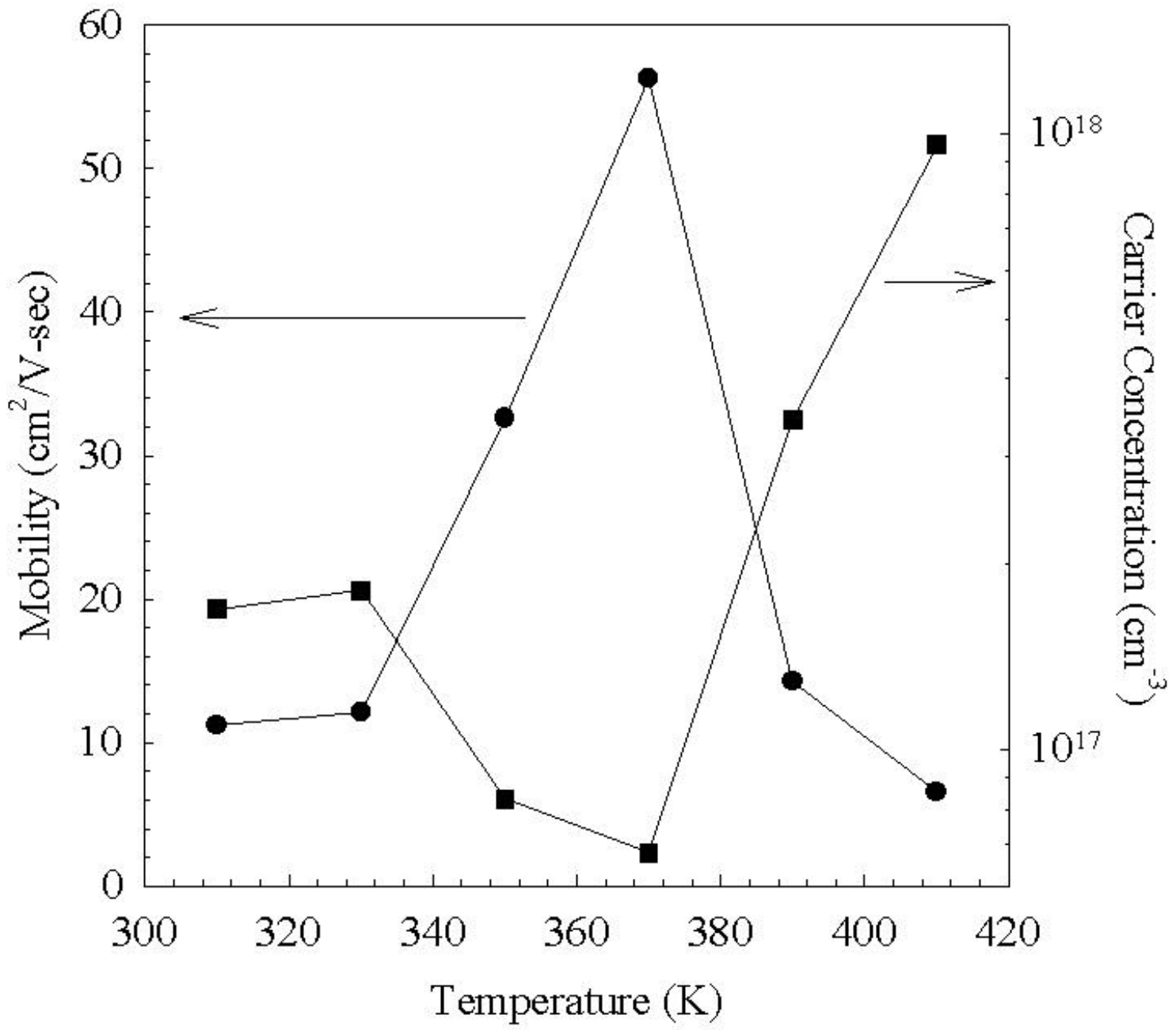

Figure 5.8 The mobility (circles) and carrier concentration (squares) vs temperature data for a Be-doped GaN layer. The wide scatter of the data represents continuing problems with electrical characterization. 


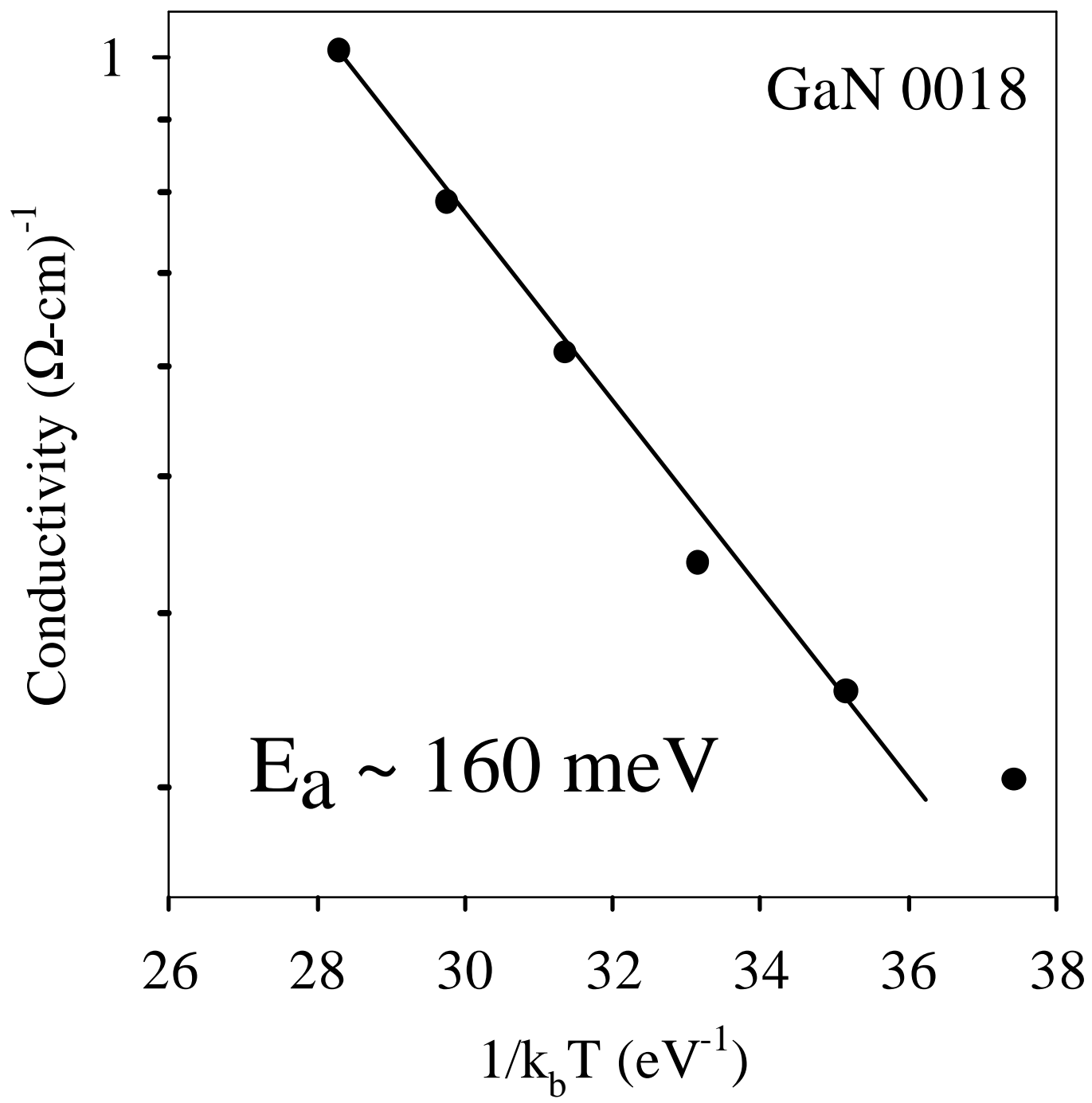

Figure 5.9 Temperature dependent conductivity of a Be-doped GaN sample. The thermal activation energy is calculated to be $\sim 160 \mathrm{meV}$. 
this way, the conductivity can be used to determine the activation energy of Be. A thermal activation energy of $160 \mathrm{meV}$ was calculated for the sample shown in Figure 5.9, roughly in agreement with other groups who report energies ranging from 130-170 meV. ${ }^{90,91}$

\subsection{Summary}

A detailed study of the incorporation of Be in GaN grown by rf-plasma assisted MBE has shown striking differences with the case of $\mathrm{Mg}$ incorporation. Be does not show as strong of a dependence on the polarity of GaN, with about two times more incorporated on the N-polarity. Incorporation is also not a strong function of substrate temperature at higher $\mathrm{Be}$ concentrations although some temperature dependence is present at low concentrations. Additionally, Be does not show a dependence on growth conditions, with incorporation rates remaining virtually unchanged when going from Ga-rich to N-stable growth. These results indicate that the incorporation of $\mathrm{Be}$ is much better behaved than for the case of $\mathrm{Mg}$ in $\mathrm{GaN}$. In addition, the effect of atomic hydrogen during growth was greatly reduced. Hydrogen was not found to significantly affect any N-polar growth in this study, while Ga-polar material only showed an effect at low Be concentrations. Hydrogen was found to incorporate at levels equal to or above the concentration of $\mathrm{Be}$ atoms in measured samples, indicative of the higher energy of the Be-H bond compared with the corresponding Mg-H bond..

Strong evidence was observed for surface accumulation of Be during growth by SIMS and Auger measurements. SIMS measurements showed an increased concentration of $\mathrm{Be}$ at the surface of the step-doped layers even though an undoped cap layer was grown in all cases. The Be steps were also out of phase with the opening and closing of the Be shutter. For the highest concentrations, the coverage of $\mathrm{Be}$ on the surface was estimated to be close to a monolayer.

Be doping was found to have a significant effect on the structure of GaN. TEM results showed threading dislocations bending away from the c-axis with the first introduction of Be into the crystal, and a severe degradation of the surface under high 
doping levels. Ga-polar material was seen to undergo a polarity inversion at Be levels of $\sim 4-5 \times 10^{19} \mathrm{~cm}^{-3}$ but, unlike the case of $\mathrm{Mg}$, significant numbers of defects were formed. Raman measurements were performed that indicate Be doping causes considerable compressive strain in the growing layer that may account for these effects.

Temperature and power-dependent PL spectra show strong evidence for donor-toacceptor and electron-to-acceptor transitions, which indicate a Be acceptor level about $100 \mathrm{meV}$ above the valence band, much shallower than the $\mathrm{Mg}$ acceptor level ( 200 meV). Hall effect measurements are less reliable, but several samples have been measured as ptype. Only one sample has shown a clear temperature dependence of electrical properties. This sample yields a thermal activation energy of approximately $160 \mathrm{meV}$. Measured hole mobilities in the p-type samples are significantly higher than for Mg doping, however samples are highly compensated. A possible mechanism for this is self-compensation by $\mathrm{Be}$ interstitials. 


\section{Chapter 6: Oxygen in Gallium Nitride}

Many groups have studied the role of oxygen in $\mathrm{GaN}$, but few careful studies of intentional oxygen doping have been performed. It is important to understand how oxygen behaves in $\mathrm{GaN}$ because it is one of the most prevalent impurities in semiconductor growth systems. Indeed, oxygen is commonly invoked as a primary cause of the residual background electron concentration in GaN. A controlled study of the incorporation, electrical properties and structural effects of oxygen needs to be performed to assess the role of oxygen in GaN. To date, most studies involving oxygen in GaN have focused on unintentionally doped material with high background levels of impurities, or material that has not been of high quality with oxygen supplied by an "uncontrolled" system background or out-diffusion from the sapphire substrate. Also of importance, and something that has not received the proper attention, is the effect of surface polarity on the incorporation of oxygen. Zywietz et al. ${ }^{100}$ used first-principles calculations to predict that, for typical Ga-stable growth conditions, the Ga-polar surface would adsorb lower amounts of oxygen than the N-polar surface. A SIMS study of Gaand N-polar GaN grown by MOCVD by Sumiya et al. ${ }^{101}$ found about 170 times more oxygen for N-polar growth. However, the N-polar material in this study was of poor quality, typical of N-polar MOCVD growth, making it difficult to determine if poor structure is the cause of the increased incorporation. It is also not clear this result is directly applicable to MBE growth of GaN.

This chapter describes the results of a controlled study of oxygen incorporation in GaN. The oxygen doped GaN layers were grown using procedures similar to the studies of $\mathrm{Mg}$ and $\mathrm{Be}$ doping. In this case, ultra high purity oxygen gas (99.998\%) was introduced through a tube directed at the substrate using an ultra-high vacuum leak valve and monitored using a residual gas analyzer as described in Chapter 2. Incorporation was studied on both N-polarity and Ga-polarity $\mathrm{GaN}$ in the same way as for the previous doping studies. 


\subsection{Incorporation of Oxygen in GaN}

Figure 6.1 shows a SIMS measurement of oxygen incorporation in a N-polar step-doped sample. During growth, the doped regions were created by adjusting the leak valve to attain the proper oxygen partial pressure. Approximately $0.25 \mu \mathrm{m}$ of doped material was grown followed by closing the oxygen valve to grow a $0.5 \mu \mathrm{m}$ undoped spacer. The partial pressures of oxygen are listed for each step in the figure. A clear dependence of the concentration of oxygen is seen with increasing oxygen partial pressure. It should also be noted that the junctions are very sharp (less than $8 \mathrm{~nm} /$ decade) for N-polarity growth. The SIMS results for a similar Ga-polar structure grown on an MOCVD template is shown in Figure 6.2. This sample showed the same trend for incorporation with oxygen partial pressure, but the steps were not as abrupt (approximately $88 \mathrm{~nm} /$ decade).

$\mathrm{N}$-type doping with oxygen was found to be controllable and reproducible under $\mathrm{Ga}$-stable growth conditions using $\mathrm{O}_{2}$ gas as the dopant source. Figure 6.3 shows the oxygen incorporation as determined by SIMS from the step-doped layers compared to room-temperature Hall effect measurements on uniformly doped layers. N-polar GaN incorporates about one out of every fifty available oxygen atoms into the growing layer based on measured partial pressures. Incorporation in Ga-polar $\mathrm{GaN}$ is less effective by about an order of magnitude. The two oxygen incorporation trend lines for Ga-polar growth indicated in Figure 6.3 were generated for different Ga overpressures. Growth conditions were found to have a dramatic influence on the incorporation of oxygen, as shown in Figure 6.4 for a Ga-polar film. During Ga-stable growth, oxygen incorporation exhibited only a small dependence on Ga overpressure. Incorporation increased about a factor of two when changing the Ga BEP from 1.0 to $1.6 \times 10^{-6}$ Torr. In contrast, however, oxygen incorporation was found to increase by up to four orders of magnitude for growth approaching N-stable conditions (approximately 5.0x $10^{-7}$ Torr Ga BEP). The dramatic increase in oxygen incorporation for a given partial pressure as growth approaches $\mathrm{N}$-stable conditions is indicated by the data contained in Table 6.1. The sharp increase with decreasing Ga flux makes oxygen incorporation difficult to control under those 


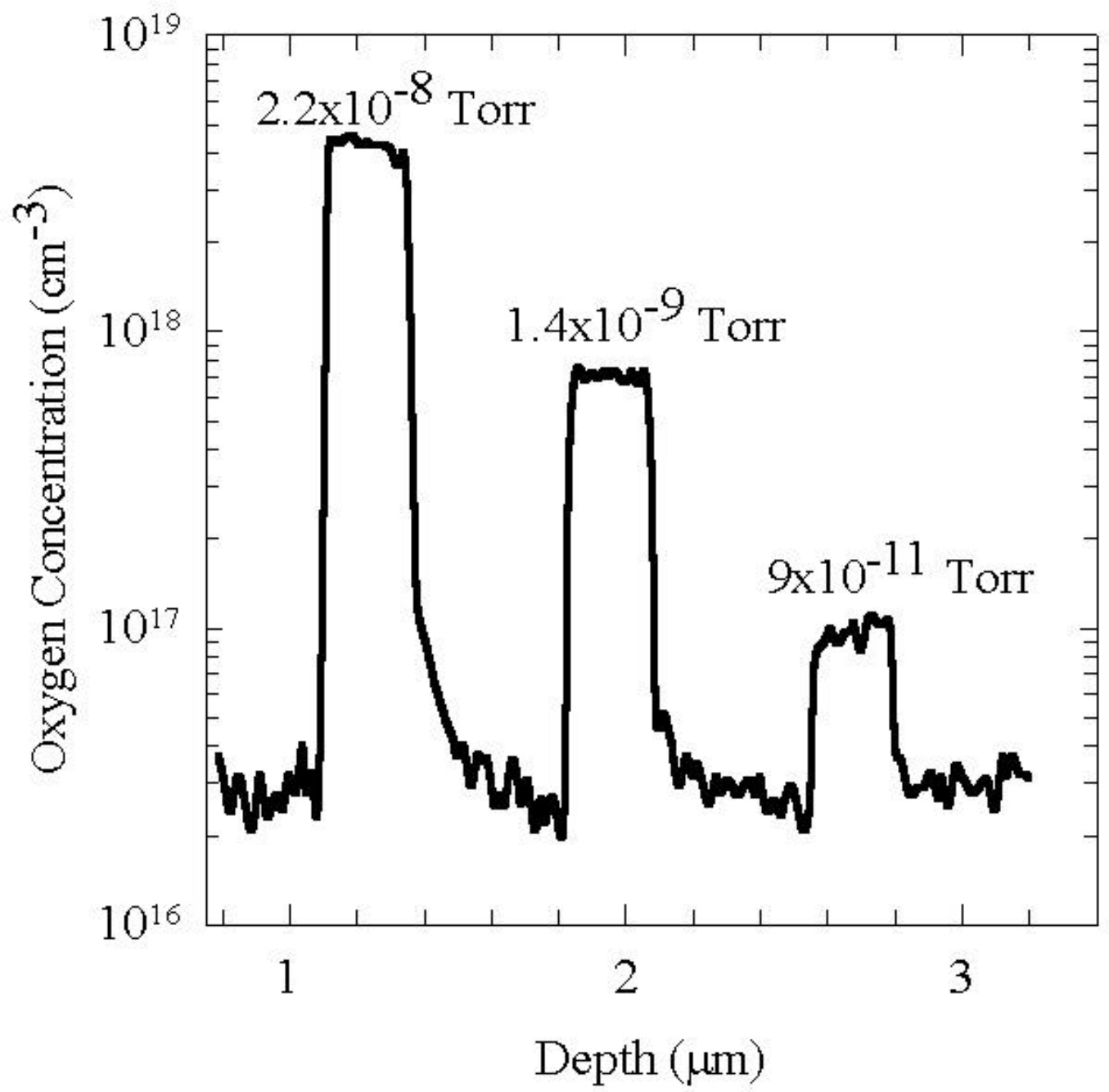

Figure 6.1 SIMS measurement showing oxygen incorporation in a N-polar step-doped sample. The partial pressure of oxygen used for each step is indicated. The steps were generated by opening and closing a leak valve. 


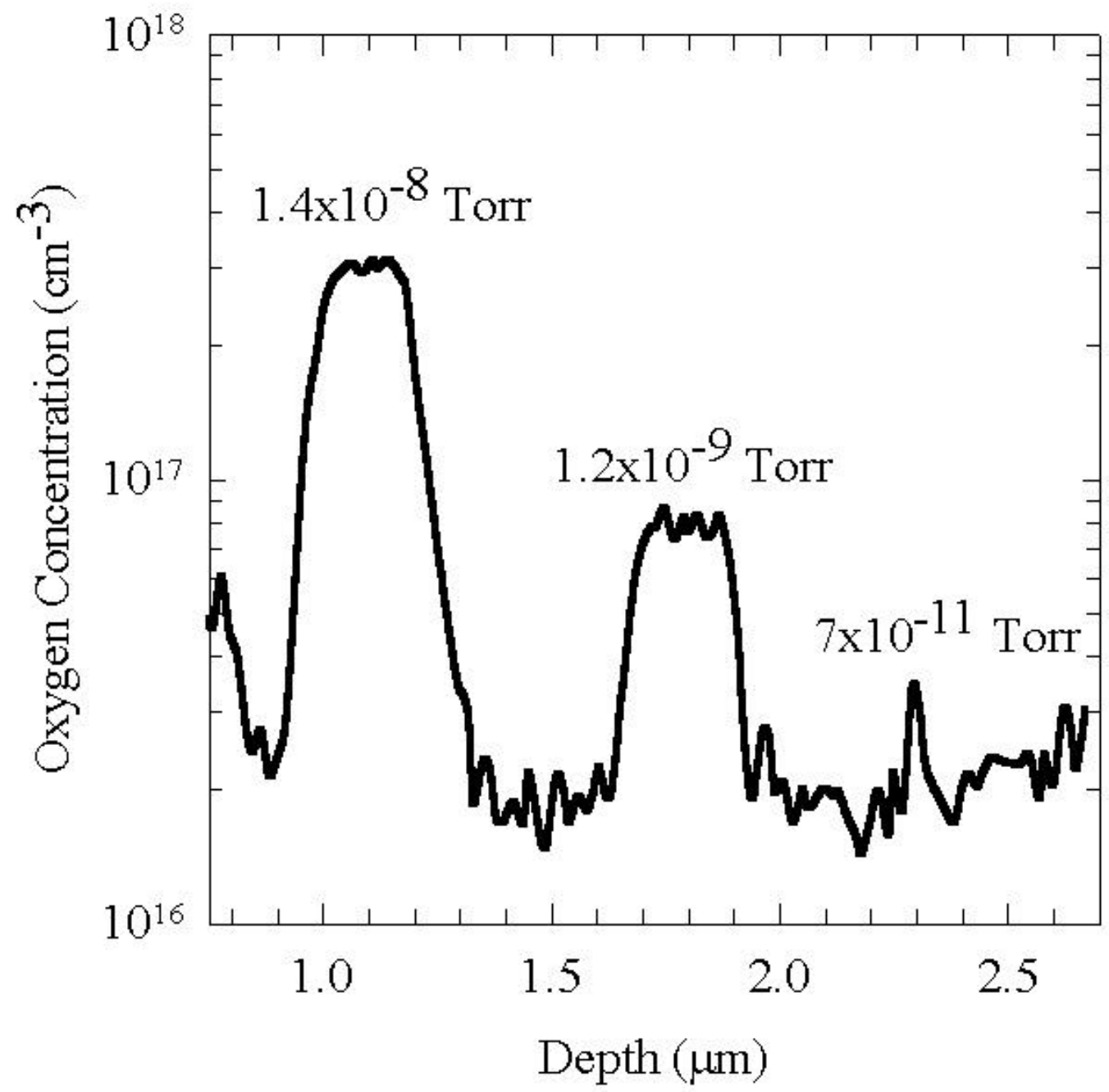

Figure 6.2 SIMS measurement showing oxygen incorporation in a Ga-polar step-doped sample. The partial pressure of oxygen used for each step is indicated. The steps were generated by opening and closing a leak valve. 


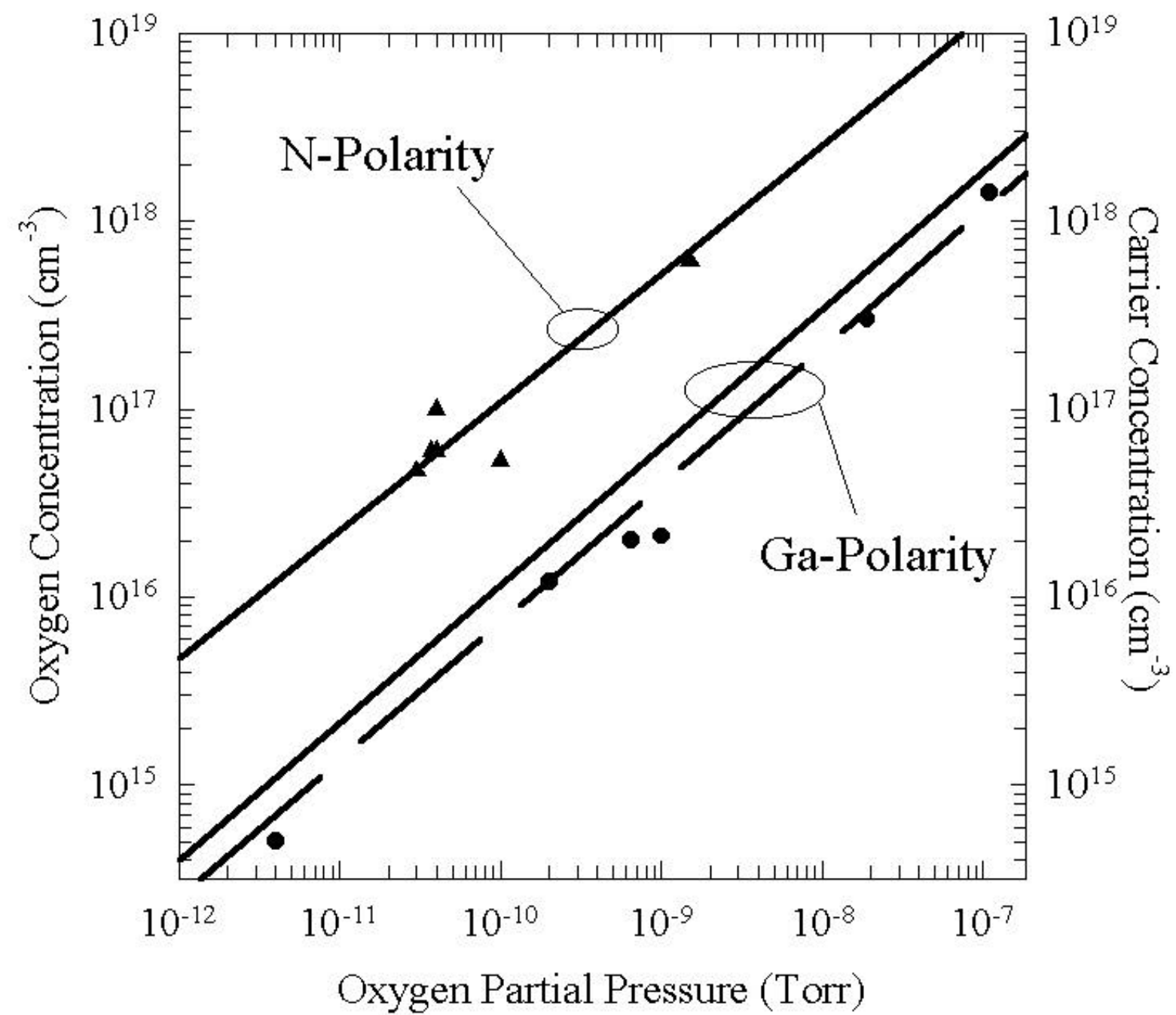

Figure 6.3 SIMS of oxygen concentration (lines) and room temperature Hall carrier concentration (points) vs oxygen partial pressure. The lines represent a least-squares fit to the SIMS oxygen concentration for constant Ga-overpressure (solid: $1.6 \times 10^{-6}$ Torr BEP, dashed: 1.0x $10^{-6}$ Torr BEP). N-polar Hall results were for layers grown under a Ga-overpressure of $1.6 \times 10^{-6}$ Torr BEP while Ga-polar Hall results were for a Ga-overpressure of $1.0 \times 10^{-6}$ Torr BEP. 


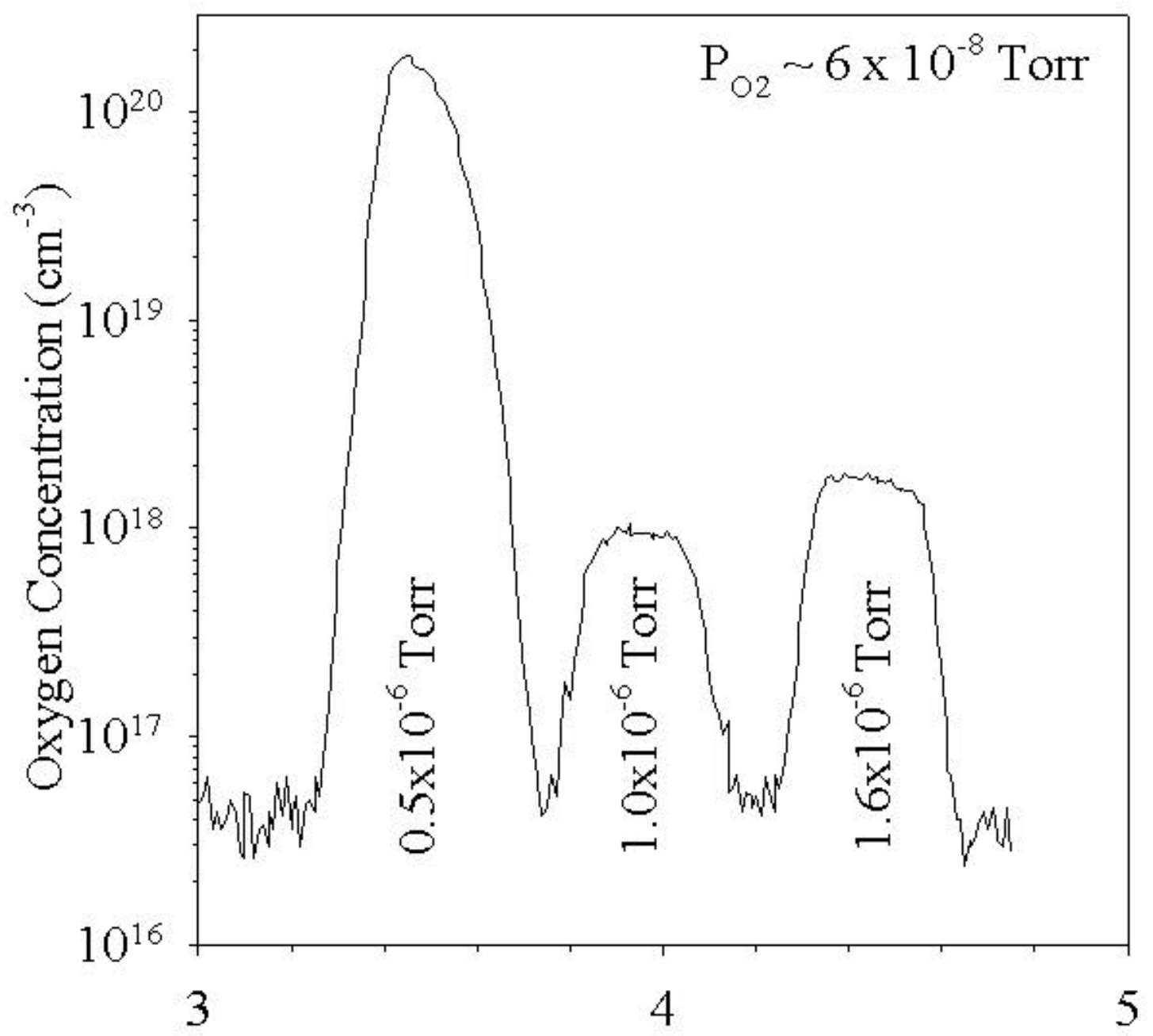

Depth $(\mu \mathrm{m})$

Figure 6.4 The variation of oxygen incorporation with Ga overpressure. The Ga BEP is indicated for each step. 
Table 6.1 The effect of Ga overpressure on oxygen incorporation.

\begin{tabular}{|c|c|c|c|c|c|}
\hline Polarity & $\begin{array}{l}\text { Ga BEP } \\
\text { (Torr) }\end{array}$ & $\mathrm{P}_{\mathrm{O} 2}$ (Torr) & $\begin{array}{l}{[\mathrm{O}]^{*}} \\
\left(\mathrm{~cm}^{-3}\right)\end{array}$ & $\begin{array}{c}\text { Carrier Density } \\
\qquad\left(\mathrm{cm}^{-3}\right)\end{array}$ & Cracks? \\
\hline $\mathrm{N}$ & $1.6 \times 10^{-6}$ & $1 \times 10^{-10}$ & & $5.4 \times 10^{16}$ & No \\
\hline $\mathrm{N}$ & $8.3 \times 10^{-7}$ & $1 \times 10^{-10}$ & & $6.3 \times 10^{17}$ & No \\
\hline $\mathrm{N}$ & $1.8 \times 10^{-6}$ & $3.0 \times 10^{-7}$ & & $3.2 \times 10^{18}$ & No \\
\hline $\mathrm{N}$ & $9.2 \times 10^{-7}$ & $1.8 \times 10^{-7}$ & & $9.0 \times 10^{19}$ & No \\
\hline $\mathrm{N}$ & $5.0 \times 10^{-7}$ & $1.7 \times 10^{-7}$ & & $6.8 \times 10^{19}$ & No \\
\hline $\mathrm{Ga}$ & $5.0 \times 10^{-7}$ & \multirow{3}{*}{$6 \times 10^{-8}$} & $1.5 \times 10^{20}$ & & \multirow{3}{*}{ No } \\
\hline $\mathrm{Ga}$ & $1.6 \times 10^{-6}$ & & $1.6 \times 10^{18}$ & & \\
\hline $\mathrm{Ga}$ & $1.0 \times 10^{-6}$ & & $8.5 \times 10^{17}$ & & \\
\hline $\mathrm{Ga}$ & $9.5 \times 10^{-7}$ & $1.0 \times 10^{-7}$ & & $1.4 \times 10^{18}$ & No \\
\hline $\mathrm{Ga}$ & $4.8 \times 10^{-7}$ & $5.0 \times 10^{-8}$ & $2.6 \times 10^{21}$ & $4.0 \times 10^{19}$ & No \\
\hline $\mathrm{Ga}$ & $4.9 \times 10^{-7}$ & $2.0 \times 10^{-7}$ & $1.5 \times 10^{22}$ & $8.4 \times 10^{19}$ & Yes \\
\hline $\mathrm{Ga}$ & $4.9 \times 10^{-7}$ & $1.5 \times 10^{-7}$ & $2.5 \times 10^{22}$ & $3.0 \times 10^{20}$ & Yes \\
\hline
\end{tabular}

* As measured by SIMS 
conditions, unlike Ga-stable growth. Note that oxygen levels as high as $2.5 \times 10^{22} \mathrm{~cm}^{-3}$ were obtained.

Oxygen incorporation results under Ga-stable conditions are consistent with the trend predicted by Van de Walle and Neugebauer, but not the absolute magnitude. ${ }^{102}$ Their calculations indicated that the formation energy of substitutional oxygen should decrease with an increasing III/V ratio for Ga-stable conditions. For conditions approaching $\mathrm{N}$-stable, the incorporation of oxygen shows a dramatic increase by up to four orders of magnitude. The difference in oxygen incorporation for different $\mathrm{Ga}$ overpressures, as well as the polarity-dependence of incorporation, can possibly be explained by considering the state of the surface during growth. One or more adlayers of Ga atoms are on the surface during Ga-stable growth conditions. ${ }^{103}$ For Ga-polar growth, these adlayers do not appear to affect oxygen incorporation as they do for N-polar growth. $^{100}$ The (0001) surface becomes less favorable for oxygen adsorption with increasing oxygen coverage, while the adsorption energy for the $(000 \overline{1})$ surface remains essentially unchanged. This is consistent with the observation that the N-polar surface incorporates more oxygen. These Ga adlayers, however, would be absent for conditions approaching $\mathrm{N}$-stable growth. Since there is no $\mathrm{Ga}$ to act as a barrier to oxygen adsorption for N-stable growth, both polarities should be highly receptive to oxygen capture. ${ }^{100}$ This agrees with the present observation of a drastic increase in incorporation for low Ga fluxes.

\subsection{Electrical Characterization}

The Hall effect results for the Ga-polar samples are for the same growth conditions as the dashed line in Figure 6.3. The carrier concentrations determined by Hall are consistent with oxygen acting as a shallow donor that remains uncompensated up to concentrations of at least $10^{18} \mathrm{~cm}^{-3}$ for each polarity of GaN. Interestingly, measured carrier concentrations from our unintentionally doped Ga polarity material correlates nicely with the residual background partial pressure of oxygen in our system during growth (approximately $4-5 \times 10^{-12}$ Torr). This strongly suggests that our residual n-type 
carrier background is due to oxygen. The SIMS and Hall effect results provide consistent evidence of the influence of polarity on the incorporation of oxygen.

The temperature-dependent Hall effect data for a Ga-polar sample is shown in Figure 6.5. Importantly, when the carrier concentration data such as that shown in this figure is analyzed using a full charge-balance equation assuming a single donor with a compensating acceptor (see Appendix B), the results indicate uncompensated material. This result is again consistent with the close correlation between Hall and SIMS results indicated in Figure 6.3. Direct analysis of the data shown in Figure 6.5 gives an activation energy of $E_{d} \sim 30 \mathrm{meV}$ for this oxygen doped sample. This energy is related to the actual energy level, $\mathrm{E}_{\mathrm{d} 0}$, of oxygen in $\mathrm{GaN}$ through the expression

$$
E_{d}=E_{d 0}-\beta N^{\frac{1}{3}}
$$

where $\mathrm{N}$ is the concentration of donors in the sample and $\beta$ is the screening constant. ${ }^{104}$ When carrier screening is taken into account using a screening constant $\beta=2.1 \times 10^{-5} \mathrm{meV}-\mathrm{cm}$ for $\mathrm{GaN}^{105}$ the donor level is determined to be $35 \mathrm{meV}$. All temperature-dependent Hall measurements to date are consistent with oxygen forming a shallow donor level with a donor energy between 35 and $39 \mathrm{meV}$. Figure 6.5 also indicates a room temperature mobility of $\sim 500 \mathrm{~cm}^{2} / \mathrm{V}$-sec and a peak low temperature value of $\sim 1500 \mathrm{~cm}^{2} / \mathrm{V}$-sec. Similar mobilities were observed for oxygen doping on both polarities of GaN. The results obtained for our oxygen-doped samples indicate a steadily declining mobility with increasing oxygen concentration, consistent with increased carrier scattering, with the mobilities slightly lower than the corresponding results for Si-doping in GaN. ${ }^{106}$ The latter may indicate that the growth conditions used for oxygen doping are not yet fully optimized.

Significant compensation occurs for concentrations above $10^{21} \mathrm{~cm}^{-3}$ as seen by comparing the oxygen incorporation and the carrier density in Table 6.1. It has been 


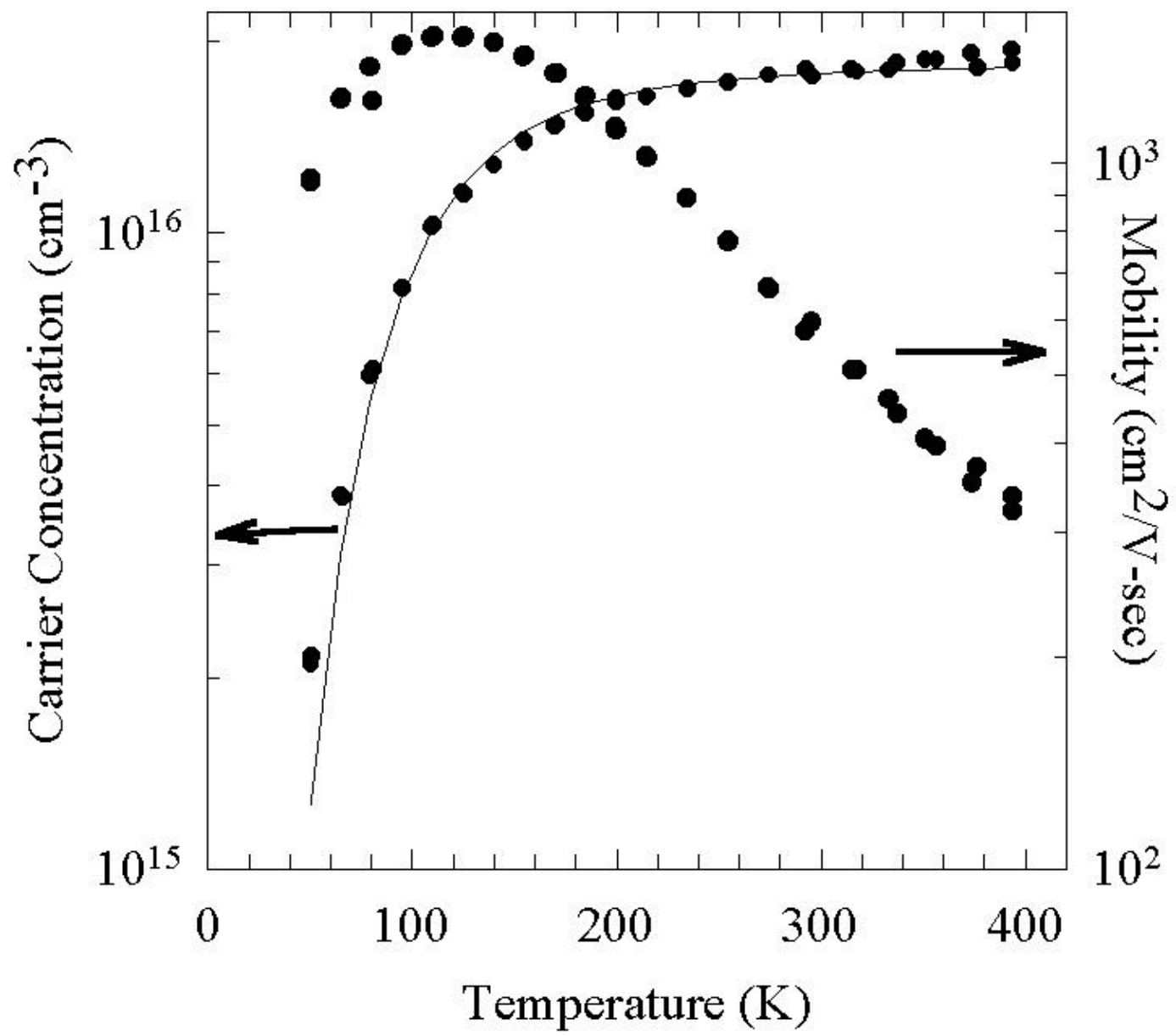

Figure 6.5 Temperature-dependent Hall effect measurement of an oxygen-doped Ga-polar sample. The line is the fit to the full charge balance equation assuming a single donor with a potential compensating acceptor. The measured donor activation energy is $30 \mathrm{meV}$ and results indicate the sample is uncompensated. 
proposed that $\mathrm{Ga}$ vacancies have a formation energy that is lower in the presence of oxygen and that significant $\mathrm{V}_{\mathrm{Ga}}-\mathrm{O}_{\mathrm{N}}$ pairs could form during growth. ${ }^{107} \mathrm{~A}$ positron annihilation study showed that $\mathrm{V}_{\mathrm{Ga}}$ formed at higher concentrations with increasing oxygen in support of this theory. ${ }^{108}$ In addition, Yang et al. showed in a photoluminescence study that $\mathrm{V}_{\mathrm{Ga}}-\mathrm{O}_{\mathrm{N}}$ pairs form an acceptor level $\sim 0.8 \mathrm{eV}$ above the valence band. ${ }^{109}$ It should be noted that our highly doped samples (oxygen levels greater than about $10^{18} \mathrm{~cm}^{-3}$ ) show a below gap absorption and appear yellow in color. It is conceivable that this absorption could be related to the formation of a $\mathrm{V}_{\mathrm{Ga}}-\mathrm{O}_{\mathrm{N}}$ complex. These complexes would act as a double acceptor and could also explain the electrical compensation seen at high doping levels.

\subsection{Structural Effects of Oxygen}

The samples with the highest incorporation of oxygen also exhibit severe cracking. Figure 6.6 shows a uv fluorescence image of a heavily cracked $\mathrm{GaN}$ sample with an oxygen concentration of approximately $2 \times 10^{22} \mathrm{~cm}^{-3}$. Similar cracking is observed for heavy $\mathrm{Si}$ doping due to tensile stress occurring for $\mathrm{Si}$ levels above $2 \times 10^{19} \mathrm{~cm}^{-3}{ }^{110}$ Raman measurements were made to measure stress in the doped films by monitoring shifts in the $\mathrm{E}_{2}$ phonon peak on the majority of the oxygen doped samples. For many Ga-polar layers, the $\mathrm{E}_{2}$ peak was first measured for the MOCVD template prior to growth for comparison with that measured for the composite system of $1 \mu \mathrm{m}$ doped material grown by MBE on about $2 \mu \mathrm{m}$ of MOCVD GaN. Table 6.2 is a compilation of the growth conditions for all samples measured by Raman, with the corresponding Raman and Hall effect results in Table 6.3. Interestingly, no evidence of increased strain was measured in uncracked layers for concentrations up to $\sim 3 \times 10^{21} \mathrm{~cm}^{-3}$. This is consistent with calculations indicating the tensile strain observed in $\mathrm{Si}$ is not due to electronic effects ${ }^{110}$ or else it would be observed in these highly doped layers. Some drastic effect does occur, however, at oxygen levels above this concentration as all layers with concentrations higher than $10^{22} \mathrm{~cm}^{-3}$ this exhibit significant cracking. 


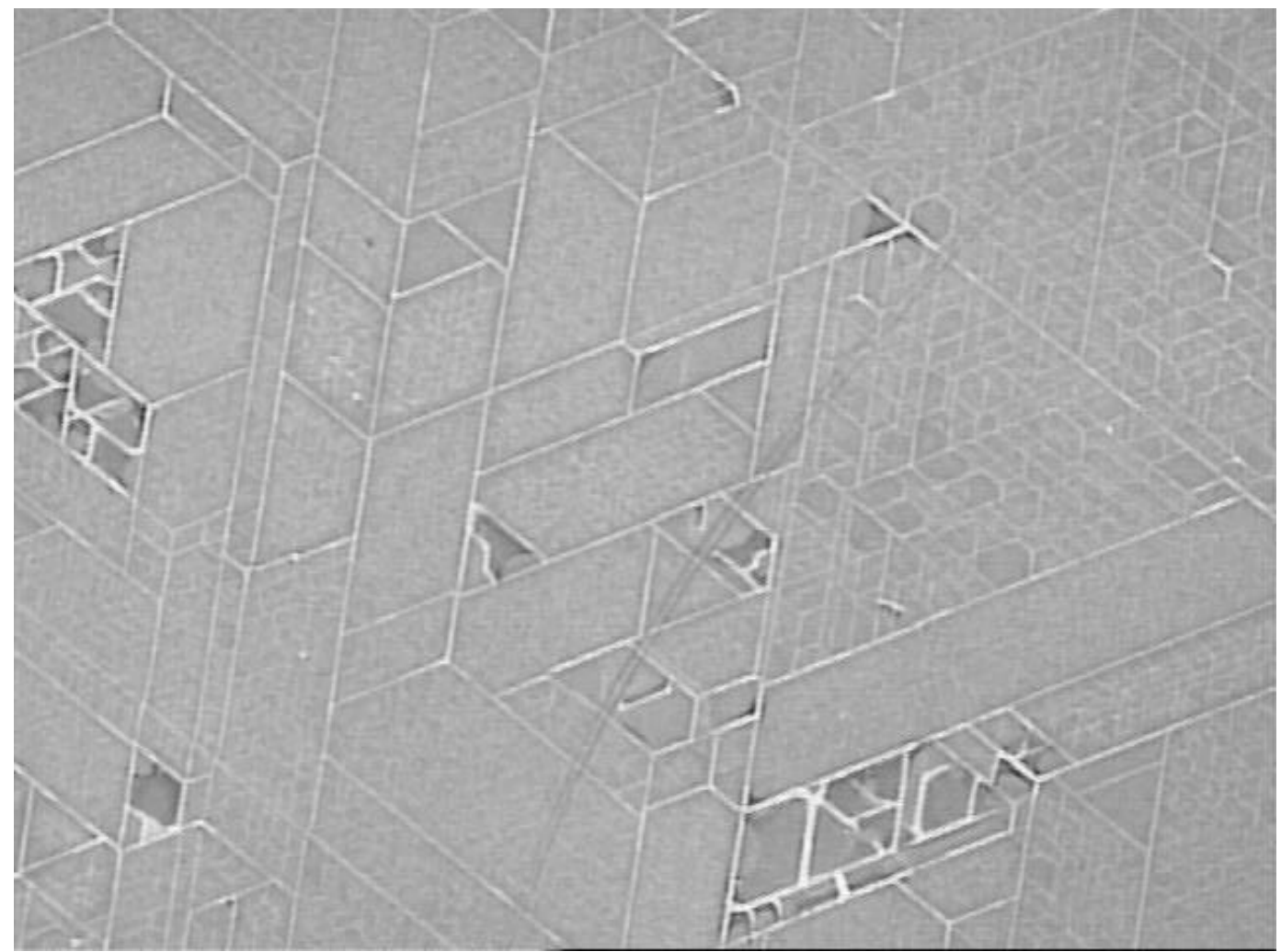

Figure 6.6 Ultraviolet fluorescence image of a heavily oxygen doped $\mathrm{GaN}$ sample. 
Table 6.2 Growth conditions for oxygen doped samples.

\begin{tabular}{|c|c|c|c|c|c|}
\hline Sample & $\mathrm{T}_{\text {sub }}$ (dial) & $\mathrm{P}_{\mathrm{O} 2}$ (Torr) & $\mathrm{P}_{\mathrm{Ga}}$ (Torr) & $\begin{array}{c}{[\mathrm{O}]} \\
\left(\mathrm{cm}^{-3}\right)\end{array}$ & $\begin{array}{c}\text { Doped Layer } \\
\text { Thickness } \\
(\mu \mathrm{m})\end{array}$ \\
\hline \multicolumn{6}{|c|}{ N-polarity, grown on sapphire } \\
\hline 00119 & 780 & Undoped & $1.6 \times 10^{-6}$ & & 1.0 \\
\hline 0154 & 780 & $5.0 \times 10^{-10}$ & $9.5 \times 10^{-7}$ & & 0.9 \\
\hline 0155 & 780 & $1.8 \times 10^{-7}$ & $9.2 \times 10^{-7}$ & & 0.9 \\
\hline 0158 & 780 & $1.7 \times 10^{-7}$ & $5.0 \times 10^{-7}$ & & 0.9 \\
\hline \multicolumn{6}{|c|}{ Ga-polarity, grown on MOCVD template, $n \sim 6 \times 10^{17}$} \\
\hline 0156 & 780 & Undoped & $9.6 \times 10^{-7}$ & $5 \times 10^{14} *$ & 0.9 \\
\hline 0157 & 780 & $2.0 \times 10^{-7}$ & $9.9 \times 10^{-7}$ & $1.5 \times 10^{18} *$ & 0.9 \\
\hline 0161 & 780 & $2.1 \times 10^{-7}$ & $4.4 \times 10^{-7}$ & $1-2 \times 10^{22} *$ & 0.9 \\
\hline \multicolumn{6}{|c|}{ Ga-polarity, grown on semi-insulating MOCVD template } \\
\hline 00105 & 780 & Undoped & $6.7 \times 10^{-7}$ & & 1.0 \\
\hline 0162 & 780 & Undoped & $1.2 \times 10^{-6}$ & $5 \times 10^{14}$ & 1.0 \\
\hline 0110 & 750 & $5 \times 10^{-8}$ & $4.8 \times 10^{-7}$ & $2.6 \times 10^{21}$ & 1.4 \\
\hline 0165 & 750 & $5.5 \times 10^{-8}$ & $4.6 \times 10^{-7}$ & & 1.0 \\
\hline 0172 & 750 & $5.0 \times 10^{-8}$ & $5.6 \times 10^{-7}$ & & 1.1 \\
\hline 0159 & 780 & $2.0 \times 10^{-7}$ & $4.9 \times 10^{-7}$ & $1.5 \times 10^{22}$ & 0.4 \\
\hline 0160 & 750 & $1.5 \times 10^{-7}$ & $4.9 \times 10^{-7}$ & $2.5 \times 10^{22}$ & 0.8 \\
\hline
\end{tabular}

* estimated from SIMS calibrations 
Table 6.3 Raman spectroscopy and Hall effect results for oxygen-doped samples.

\begin{tabular}{|c|c|c|c|c|}
\hline Sample & $\begin{array}{c}\mathrm{E}_{2} \text { peak position } \\
\left(\mathrm{cm}^{-1}\right)\end{array}$ & $\begin{array}{c}\text { Mobility } \\
\left(\mathrm{cm}^{2} / \mathrm{Vs}\right)\end{array}$ & $\begin{array}{c}\text { Density } \\
\left(\mathrm{cm}^{-3}\right)\end{array}$ & Cracks? \\
\hline \multicolumn{5}{|c|}{ N-polarity, grown on sapphire } \\
\hline 00119 & 570.3 & $\sim 220$ & $\sim 1 \times 10^{15}$ & No \\
\hline 0154 & 569.6 & 210 & $1.7 \times 10^{18}$ & No \\
\hline 0155 & 570.2 & 40 & $9.0 \times 10^{19}$ & No \\
\hline 0158 & 570.5 & 20 & $6.8 \times 10^{19}$ & \\
\hline
\end{tabular}

\begin{tabular}{|c|c|c|c|c|c|}
\hline Sample & $\begin{array}{c}\mathrm{E}_{2} \text { peak } \\
\text { position }\left(\mathrm{cm}^{-1}\right)\end{array}$ & $\begin{array}{l}E_{2} \text { peak } \\
\text { substrate }\end{array}$ & $\begin{array}{l}\text { Mobility } \\
\left(\mathrm{cm}^{2} / \mathrm{Vs}\right)\end{array}$ & $\begin{array}{l}\text { Density } \\
\left(\mathrm{cm}^{-3}\right)\end{array}$ & Cracks? \\
\hline \multicolumn{6}{|c|}{ Ga-polarity, grown on MOCVD template, $n \sim 6 \times 10^{17}$} \\
\hline 0156 & 569.2 & 569.0 & & & No \\
\hline 0157 & 569.0 & 569.0 & & & No \\
\hline 0161 & 569.2 & 569.2 & & & Yes \\
\hline \multicolumn{6}{|c|}{ Ga-polarity, grown on semi-insulating MOCVD template } \\
\hline 00105 & 571.0 & 570.6 & 30 & $\sim 4 \times 10^{14}$ & No \\
\hline 0162 & 571.7 & 571.5 & 365 & $3.8 \times 10^{17}$ & No \\
\hline 0110 & 570.5 & 570.6 & 65 & $4.0 \times 10^{19}$ & No \\
\hline 0165 & 571.8 & 571.6 & 18 & $9.0 \times 10^{19}$ & Yes \\
\hline 0172 & 571.4 & 571.5 & & & No \\
\hline 0159 & 571.5 & 571.5 & 17 & $8.4 \times 10^{19}$ & Yes \\
\hline 0160 & 571.7 & 571.5 & 10 & $3.0 \times 10^{20}$ & Yes \\
\hline
\end{tabular}


In contrast to the high-doping case, however, low concentrations of oxygen appear to be beneficial during the initial stages of GaN growth. An AFM study of oxygen doped N-polar material indicated the appearance of smooth steps that are not normally observed for the standard growth conditions in this system. The addition of low levels of oxygen during low temperature buffer layer growth enhanced the coalescence of nucleation islands and led to smooth films. This can be seen from the AFM images in Figure 6.7. The image in Figure 6.7a is from a sample that was grown with an approximate oxygen concentration of $10^{16} \mathrm{~cm}^{-3}$ during buffer layer and layer growth. Figure $6.7 \mathrm{~b}$ displays an image from a sample grown at a similar oxygen concentration, but the oxygen was turned on after the buffer layer was completed. The sample with the oxygen doped buffer layer shows a surface with far fewer features. Note also that the height scales for the two images are correspondingly different.

\subsection{Summary}

A controlled study of oxygen incorporation on high quality $\mathrm{GaN}$ was performed by MBE. SIMS and Hall effect results show a distinct surface polarity dependence, with the $(000 \overline{1})$ polarity incorporating about an order of magnitude more oxygen than the (0001) polarity. This is the first time that this polarity difference has been shown unambiguously. Oxygen also shows an incorporation dependence on the Ga overpressure used during growth. Incorporation during Ga-stable conditions has a small dependence on Ga flux with more oxygen incorporated with increasing Ga BEP. A drastic incorporation increase is observed when approaching $\mathrm{N}$-stable conditions. This effect is explained by taking into account the effect of the Ga adlayers that exist on the surface during Ga-stable growth.

Oxygen is an effective and controllable donor impurity. Uncompensated, unstrained material with carrier concentrations up to $10^{18} \mathrm{~cm}^{-3}$ have been obtained although significant strain and electrical compensation do occur at higher levels. Significant cracking of the samples is observed at higher doping levels. Electrical 


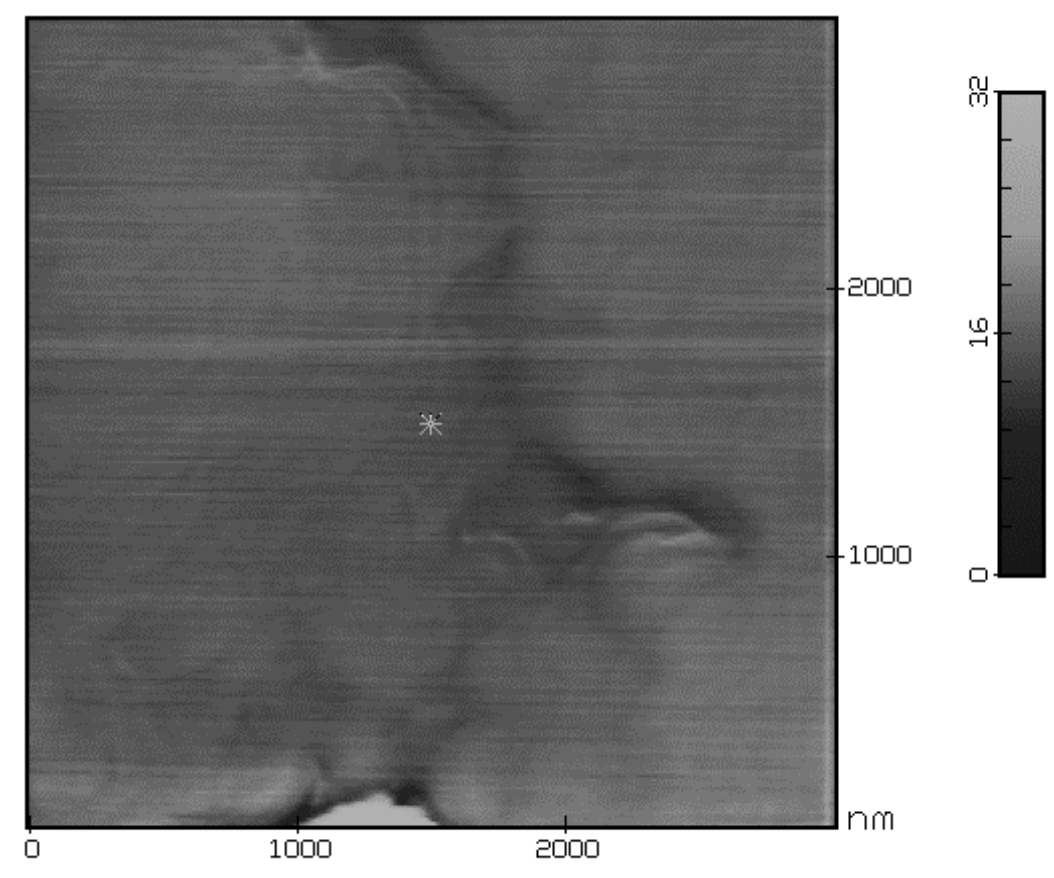

a)

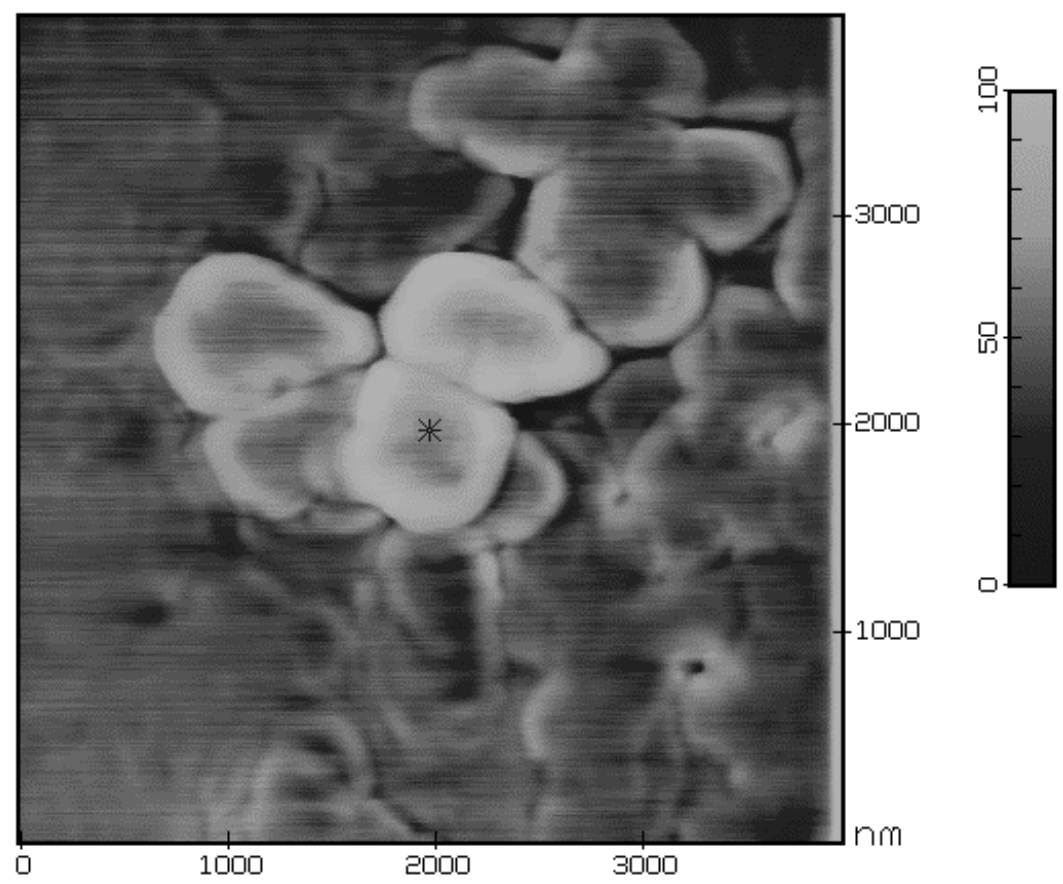

b)

Figure 6.7 AFM images from oxygen doped samples. The sample in a) was grown with an oxygen doped buffer layer while the sample in b) had an undoped buffer layer. Note the differences in the height scales (in $\mathrm{nm}$ ). 
measurements indicated that doped material is of high quality with reasonable values for mobilities on both polarities. Low concentrations of oxygen during low temperature buffer layer growth aid in the coalescence of GaN nucleation islands, leading to smooth films. 


\section{Chapter 7: Conclusions and Work Proposed for the Future}

\subsection{Conclusions}

In this study, growth kinetics and doping issues in $\mathrm{GaN}$ were studied in great detail by rf-plasma assisted molecular beam epitaxy. The most important results are listed here.

1) Control was established over the dominant active nitrogen specie emitted from two different rf-plasma sources. The Oxford CARS-25 source could provide an active flux consisting mainly of either atoms, a mixture of atoms and low-energy ions, or high-energy ions. The EPI Unibulb source produced a significant amount of metastable molecular nitrogen.

2) The reactivity of each active nitrogen specie was determined by the nitridation of sapphire. The nitridation rate for high-energy ions was about three times larger than nitridation by atomic nitrogen at a similar concentration. A mixture of atomic nitrogen and low energy ions showed an initiation stage followed by a slower nitridation rate. Metastable nitrogen does not appear to be very reactive for nitridation.

3) Boron contamination originating from the PBN liners of the plasma sources was observed during the nitridation of sapphire and growth of GaN. The formation of $\mathrm{Al}_{1-\mathrm{x}} \mathrm{B}_{\mathrm{x}} \mathrm{N}$ alloys can explain the observation of smaller than expected lattice constants for AlN formation during the sapphire nitridation.

4) Studies of growth rate as a function of temperature suggest that the $\mathrm{GaN}$ surface is prone to "attack" by neutral and ionic atomic nitrogen above $700^{\circ} \mathrm{C}$, promoting enhanced decomposition. Growth using neutral metastable molecular nitrogen results in a temperature-dependent growth rate more consistent with predicted thermal decomposition rates. 
5) Growth under an atomic hydrogen flux stabilizes the growing surface for $\mathrm{N}$-polarity growth. Too much hydrogen may promote Ga desorption, possibly due to competition for $\mathrm{N}$ bonds.

6) Growth with predominantly metastable nitrogen resulted in improved electrical quality. A dramatic increase in Hall mobility was observed for growth with the EPI Unibulb source. Metastable molecular nitrogen is preferable to neutral or ionic atomic nitrogen for MBE growth.

7) There is a significant dependence of $\mathrm{Mg}$ incorporation on polarity with the Ga-polarity incorporating a factor of up to twenty-five times more $\mathrm{Mg}$ than the N-polarity under the Ga-rich growth conditions used.

8) Calculations predicting that hydrogen enhances the incorporation of $\mathrm{Mg}$ in $\mathrm{GaN}$ were verified experimentally. Although hydrogen enhances incorporation for both polarities of $\mathrm{GaN}$, it does not eliminate the polarity dependence.

9) $\mathrm{Mg}$ forms a surface accumulation layer with a stable configuration of close to a monolayer. Only a small fraction of the actual surface $\mathrm{Mg}$ is incorporated while the remainder segregates to the new surface.

10) Puzzling results in the literature concerning p-type doping with $\mathrm{Mg}$ have been explained using the results generated in this study. Anomalous reports of a dramatic decrease in p-type conductivity with increasing $\mathrm{Mg}$ flux have been explained by the effects of polarity dependence and polarity inversion.

11) The incorporation of $\mathrm{Be}$ in $\mathrm{GaN}$ is much better behaved than for the case of Mg. Be does not show a strong dependence on the polarity of GaN, with about two times more incorporated on the N-polarity. Incorporation is also not a strong function of substrate temperature at higher Be concentrations, although some temperature dependence is present at low concentrations. Additionally, Be does not show a 
dependence on growth conditions, with incorporation rates remaining virtually unchanged when going from Ga-rich to N-stable growth.

12) Hydrogen was not found to significantly affect Be incorporation during N-polar growth, while Ga-polar material showed a slight incorporation enhancement at low Be concentrations. This result conflicts with calculations that predict hydrogen should enhance the solubility of Be in GaN.

13) Hydrogen was found to incorporate at levels equal to or above the concentration of $\mathrm{Be}$ atoms for layers grown under atomic hydrogen in measured samples, indicative of the higher energy of the Be- $\mathrm{H}$ bond compared with the corresponding $\mathrm{Mg}-\mathrm{H}$ bond.

14) Be forms a surface accumulation layer during growth. For the highest concentrations, the coverage of $\mathrm{Be}$ on the surface was estimated to be close to a monolayer.

15) Be doping was found to have a significant effect on the structure of GaN. TEM results showed threading dislocations bending away from the c-axis with the first introduction of Be into the crystal, and a severe degradation of the surface under high doping levels.

16) PL measurements show that Be forms an acceptor level about $100 \mathrm{meV}$ above the valence band. Hall effect measurements indicate that selected samples are p-type but suffer from significant compensation. Measured hole mobilities in the p-type samples are significantly higher than for $\mathrm{Mg}$ doping. Only one sample to date has allowed the measurement of a thermal activation energy, which was determined to be approximately $160 \mathrm{meV}$. 
17) A controlled study of oxygen incorporation on high quality $\mathrm{GaN}$ indicates a distinct surface polarity dependence, with the $(000 \overline{1})$ polarity incorporating about an order of magnitude more oxygen than the (0001) polarity.

18) Oxygen incorporation depends on the $\mathrm{Ga}$ overpressure used during growth. Incorporation during Ga-stable conditions has a weak dependence on Ga flux with more oxygen incorporated with increasing Ga BEP. A drastic incorporation increase is observed when approaching N-stable conditions.

19) Oxygen doping is effective and controllable resulting in uncompensated material with carrier concentrations up to $10^{18} \mathrm{~cm}^{-3}$. Electrical measurements indicated that doped material is of high quality with reasonable values for mobilities on both polarities.

20) Significant stress occurs at high incorporation indicated by the observation of cracking in oxygen doped samples for concentrations higher than about $3 \times 10^{21} \mathrm{~cm}^{-3}$.

\subsection{Future Work}

One extension of the current work is in the area of co-doping. In principle, it should be possible to incorporate clusters of impurities that behave as a p-type dopant. The pairing of a donor and two acceptors should yield one electrically active hole, and should present a decreased scattering cross section compared to an isolated ionized impurity, thereby increasing hole mobility. At least one group has shown promising results from co-doping in $\mathrm{GaN}{ }^{111}$ In that study, cubic $\mathrm{GaN}$ was doped with $\mathrm{Be}$ and $\mathrm{O}$ that resulted in hole concentrations of approximately $5 \times 10^{18} \mathrm{~cm}^{-3}$ with a maximum room temperature hole mobility of about $150 \mathrm{~cm}^{2} / \mathrm{V}$-sec. Figure 7.1 shows the SIMS measurement of a Ga-polar sample that has been co-doped with $\mathrm{Be}$ and $\mathrm{O}$. The oxygen partial pressure was maintained at $1 \times 10^{-9}$ Torr throughout the region shown in the figure. It can be seen that for low fluxes of Be, very little oxygen incorporates. When higher fluxes of Be are used, however, a dramatic increase in oxygen incorporation occurs. In fact, at the highest concentrations, a "magic" 2 to 1 ratio of $\mathrm{Be}$ to $\mathrm{O}$ exists. This is 


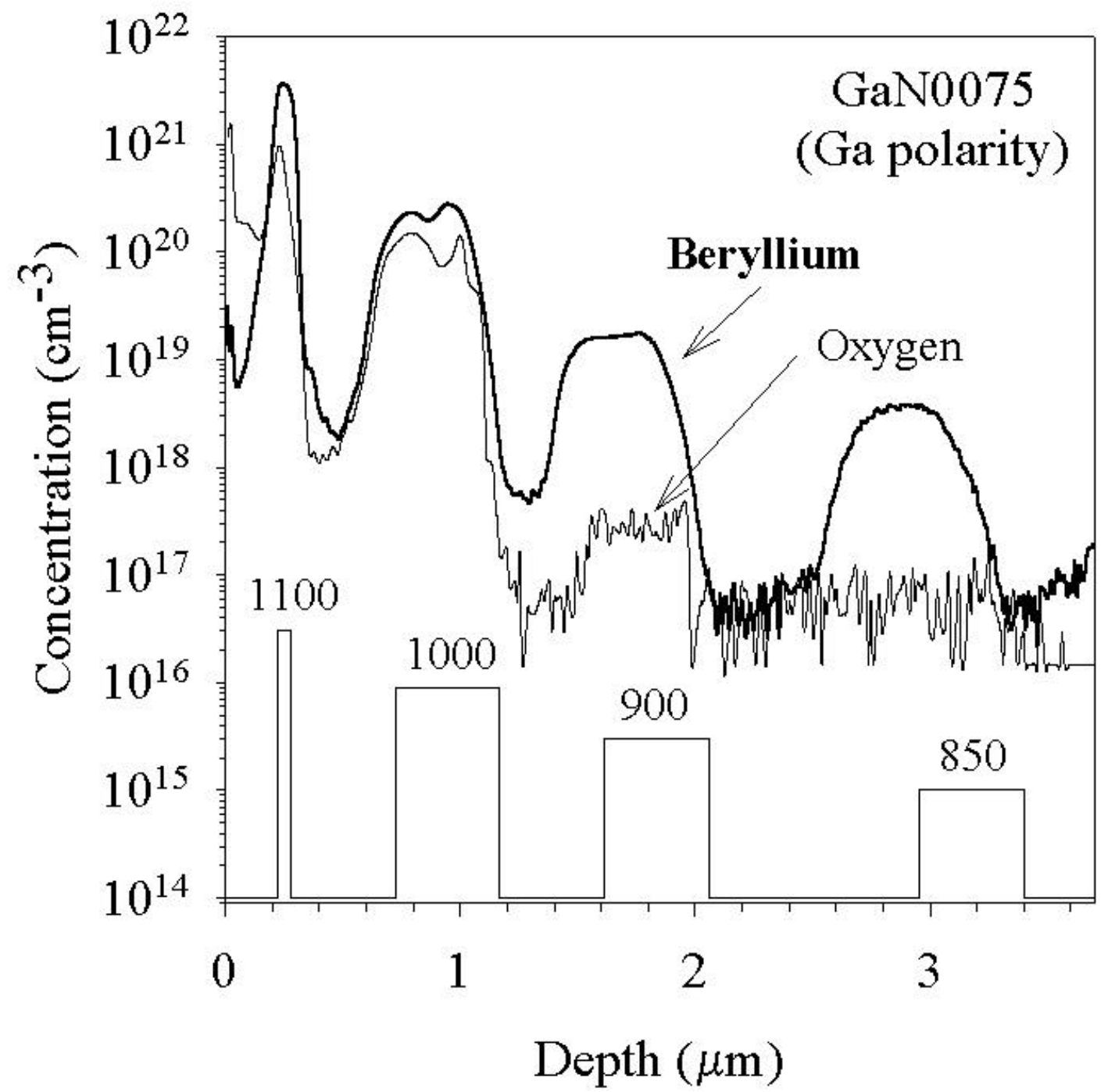

Figure 7.1 SIMS measurement of a step-doped sample that was co-doped with Be and $\mathrm{O}$. A dramatic increase of oxygen incorporation was observed for high Be fluxes. The partial pressure was held constant during the growth. Be oven temperatures are indicated for each step. 
precisely what is needed to achieve p-type doping. Be was also incorporated at levels far in excess of the limits observed during the study of Be incorporation (see Chapter 5).

Several uniform co-doped samples were grown under widely varying growth conditions to exploit this fortuitous effect. Unfortunately, Hall effect results indicated that every Ga-polar sample was electrically insulating while the only N-polar sample grown was highly n-type. In spite of these initial disappointing results, the idea of co-doping in GaN should not be abandoned just yet. Future research should focus on alternative co-doping schemes. The study of the more established p-type dopant $\mathrm{Mg}$ as the partner to $\mathrm{O}$ is an excellent next step. The incorporation characteristics of $\mathrm{Mg}$ in the presence of an oxygen background should be studied. If this incorporation study shows promise, uniformly doped layer should be grown to determine the electrical activity of the co-dopants.

$\mathrm{GaN}$ is a remarkable material that will continue to expand into a great number of markets worldwide. Further basic research will continue to improve the quality of material and help to solve some of the more troubling growth issues such as p-type doping. It is the sincere hope of the author that this work will serve as a foundation for further studies and improvement of GaN. 


\section{Appendix A: $\quad$ Basic Procedures}

\section{A.1. Sapphire Substrate Preparation}

The following is the standard degreasing procedure used for all substrates. Approximately $50 \mathrm{~mL}$ of each solvent is used at $\sim 80^{\circ} \mathrm{C}$.

Trichloroethylene (TCE): 5 minutes

Trichloroethylene (TCE) 5 minutes

Acetone 2 minutes

Methanol 5 minutes

Methanol 5 minutes

After the last dip in methanol, the sample is removed and immediately blown dry with ultra high purity (UHP) nitrogen gas. When the sapphire substrate has been degreased, it is placed in a $3: 1 \mathrm{H}_{2} \mathrm{SO}_{4}: \mathrm{H}_{3} \mathrm{PO}_{4}$ solution that is held at $\sim 160^{\circ} \mathrm{C}$ for 10 minutes. This solution is contained in a quartz beaker and the acid temperature is measured directly by a teflon-coated thermocouple. The sample and beaker are then placed in a water-cooling bath for an additional 5 minutes. After the chemical etch, the substrate is rinsed under flowing deionized (DI) water $(>18 \mathrm{M} \Omega-\mathrm{cm})$ for five minutes. The substrate is then immediately blown dry with UHP nitrogen gas. This procedure has been determined within our laboratory to produce surfaces that are flat to within the resolution of our AFM set-up.

The substrate is mounted on the substrate block using a 52\%:48\% indium tin eutectic. This eutectic offers the wetting properties of indium and the lower vapor pressure of tin. Since tin has a lower vapor pressure, it will not evaporate as quickly as indium at a given temperature. This is important as indium alone evaporates after only a matter of hours at typical $\mathrm{GaN}$ growth temperatures. After the substrate has been mounted, the block is placed into the load lock attached to the growth system. The load 
lock is evacuated to a pressure less than $1 \times 10^{-7}$ Torr before it is transferred to the growth chamber.

\section{A.2. MOCVD- and HVPE-Grown GaN Substrate Preparation}

MOCVD- and HVPE-grown GaN substrates are used to investigate Ga-polarity GaN growth. These substrates are degreased in the same manner as described above for sapphire. Once a substrate is degreased, it is placed in a 1:1 $\mathrm{HCl}: \mathrm{DI} \mathrm{H}_{2} \mathrm{O}$ solution at room temperature for 5 minutes. After this chemical cleaning procedure, the substrate is placed under flowing DI water for 5 minutes. The substrate is then immediately blown dry with UHP nitrogen gas. The GaN substrates are mounted on the substrate block and loaded into the growth system in the same way as described above for sapphire.

\section{A.3. In Situ Substrate Cleaning and Relative Temperature Check}

Once the substrate has been transferred into the growth chamber, it is heated to a substrate dial temperature of $750^{\circ} \mathrm{C}$. Atomic hydrogen is used to clean all types of substrate used in this study. Atomic hydrogen has been shown to be very effective at removing surface contamination on semiconductors. The substrate is cleaned with $\sim 2 \times 10^{-6}$ Torr BEP of hydrogen for 20 minutes.

At this time when using sapphire, it is possible to check the relative temperature of the substrate by determining the condensation temperature of $\mathrm{Ga}$ on sapphire. Finding the dial temperature at which Ga condenses on the clean sapphire surface can determine the relative temperature of the sample. By comparing the Ga condensation temperature, $\mathrm{T}_{\text {cond, }}$, from day-to-day, it is possible to minimize the effect of the changes in the block's emissivity. It is also useful in that $\mathrm{T}_{\text {cond }}$ depends on the temperature of the sample surface, not the temperature at the monitoring thermocouple.

After the completion of hydrogen cleaning, the substrate is exposed to a Ga flux of $1.3 \times 10^{-6}$ Torr BEP while the temperature is slowly lowered. The actual flux used is not important as long as the flux is the same from day-to-day. Condensation is a 
competition between how much material is arriving and how much is reflected or desorbed. If the amount of arriving material is changed (by using a different flux), the condensation temperature will also change. The substrate temperature is lowered until the RHEED pattern disappears, indicative of Ga condensation. This temperature can then be compared to previous growths to determine if any adjustment in growth parameters is necessary. It is known from this method that the temperature of the sample can decrease by up to $30^{\circ} \mathrm{C}$ when going from a clean to a coated block due to the change in emissivity. Once the block is coated, however, a change of $10^{\circ} \mathrm{C}$ between growth runs is considered large. Most day-to-day variation is limited to less than $\pm 5^{\circ} \mathrm{C}$.

\section{A.4. Buffer Layer Growth}

The growth of a buffer layer on sapphire for GaN growth is very important due to the large thermal and lattice mismatches involved between $\mathrm{GaN}$ and sapphire. Low temperature buffer layers have been shown to lead to high-quality N-polarity GaN. Numerous studies have been performed on the optimization of buffer layers, but many of the results appear to be system dependent. ${ }^{112,113,114,115}$ Few common threads are seen between these studies and the growth of buffer layers remains something of an art. One common thread amongst the various buffer layer studies is the use of a large Ga flux, almost to the point of condensation. The other is that while these buffer layers are grown at "low temperature", meaning lower than the growth temperature, with higher "low" temperatures tending to increase the size of nucleation islands.

Once $\mathrm{T}_{\text {cond }}$ has been determined, it is necessary to remove the excess $\mathrm{Ga}$ and return the sapphire to its original, pristine state. This is done by thermally desorbing the Ga. The substrate temperature is raised approximately $50^{\circ} \mathrm{C}$ until the RHEED pattern returns to the bright $1 \times 1$ pattern ( 5 minutes). Once this has occurred, the substrate temperature is lowered to a point $10^{\circ} \mathrm{C}$ above $\mathrm{T}_{\text {cond }}$. Care must be taken with the ramp rate so that the actual temperature of the substrate does not fall below $\mathrm{T}_{\text {cond }}$ while the temperature stabilizes. 
At this point, the Ga should be open to the substrate $\left(1.3 \times 10^{-6}\right.$ Torr BEP), and the nitrogen source should be operating, but shuttered. The $\mathrm{N}$ source parameters used are $200 \mathrm{~W}, 0.6 \mathrm{sccm}$. This gives a growth rate during N-limited growth of $\sim 0.15 \mu \mathrm{m} / \mathrm{hr}$ for a source-to-substrate distance of $\sim 24 \mathrm{~cm}$. To start buffer layer growth, the $\mathrm{N}$ shutter is opened.

Figure A.1 shows how the RHEED pattern changes during buffer layer growth. When the $\mathrm{N}$ shutter is first opened, an immediate change should be seen in the RHEED. If the sapphire is charged due to the electron beam, the charging will go away. The streaks will dim and it will be apparent that the azimuth is changing by $30^{\circ}$. The change in azimuth is due to the orientation of $\mathrm{GaN}$ on sapphire. The [0001] directions of both materials are aligned, but the symmetry directions perpendicular to the c-axis are rotated by $30^{\circ}$ with respect to each other. ${ }^{116}$ This will be followed by Ga condensation due to Ga having a higher $\mathrm{T}_{\text {cond }}$ on $\mathrm{GaN}$ than on sapphire. The Ga shutter is closed after one minute of growth, leaving the $\mathrm{N}$ shutter open, to allow the condensed Ga to either desorb or react with the active nitrogen.

After one minute of growth interruption, the Ga shutter is re-opened. Again, Ga will condense, but not as quickly as during the first growth stage. This is most likely due to the smoothing of the film and the lack of places for $\mathrm{Ga}$ to attach to the surface. The RHEED pattern should begin to take on the appearance of "beaded streaks" before it is lost under the condensed Ga. Growth is continued for one minute, followed by a one minute growth interruption with the Ga shutter closed.

This pattern of opening and closing the Ga shutter should be completed a total of five times (five growth stages and five growth interruptions). After the $5^{\text {th }}$ growth stage, the $\mathrm{N}$ shutter is closed instead of the Ga shutter to terminate the buffer layer. The RHEED pattern from the buffer layer should continue to improve during the 5 stages, with less Ga condensation occurring. Even with the $\mathrm{N}$ shutter closed after buffer layer growth and the Ga shutter open, no condensation should occur. The RHEED pattern should be unreconstructed and streaky. 


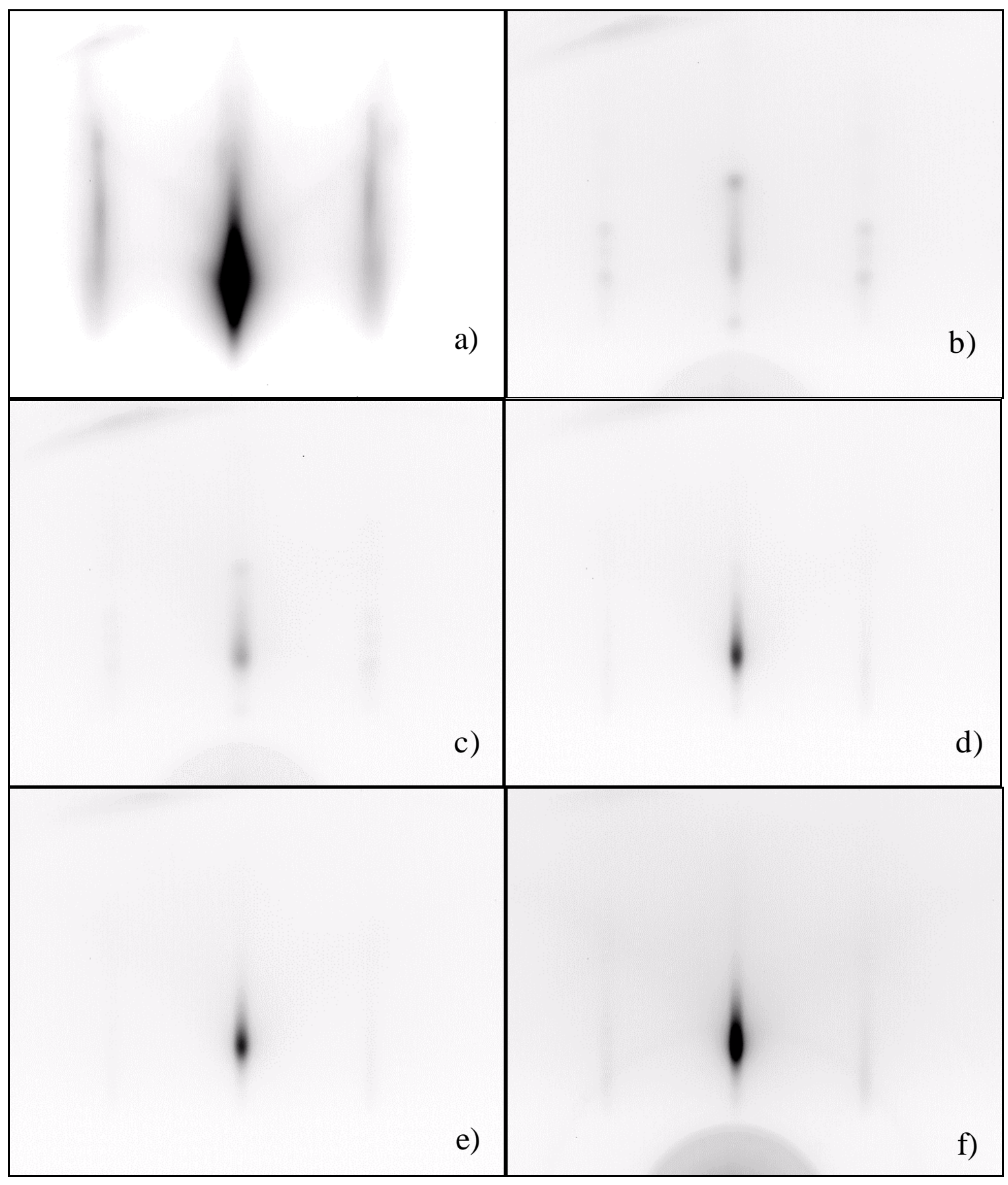

Figure A.1 RHEED images of the buffer layer growth. a) The [1 100$]$ direction of bare sapphire. b) The [11 $\overline{2} 0$ ] direction of $\mathrm{GaN}$ after the first minute of growth and the first one minute growth interruption. The pattern is faint and broken. c) After the second growth interruption. d) After the third growth interruption. The pattern is becoming bright and streaky. e) After the fourth growth interruption. f) The completed buffer layer. 
Following the termination of the buffer layer, the sample is heated to the growth temperature under a $\mathrm{Ga}$ flux. The $\mathrm{Ga}$ and nitrogen fluxes are re-set to the necessary levels, and once stabilized, layer growth is commenced. 


\section{Appendix B: The Hall Effect}

The Hall Effect was discovered by E.H. Hall in 1879 and results from magnetic forces acting on currents flowing through a conducting material that has been placed in a magnetic field. Suppose current flows along the $\mathrm{x}$-direction of a conducting material that has been placed in a uniform magnetic field such that the z-axis of the material lines up with the field (See Figure B.1). The simplest case assumes that the current is only due to one type of charge carrier, for example, electrons. If the current is in the positive $\mathrm{x}$ direction, then the electrons are travelling along the $-\mathrm{x}$-axis. The motion of these electrons is defined by the Lorentz force, given by

$$
\mathbf{F}=\mathrm{q}(\mathbf{E}+\mathbf{v x} \mathbf{B})
$$

where $\mathrm{q}$ is the charge of the electron, $\mathbf{E}$ and $\mathbf{B}$ are the electric and magnetic fields, respectively, and $\mathbf{v}$ is the velocity of the electron. As the electrons move through the material, they experience an acceleration in the negative y-direction due to the magnetic force, and charge begins to build at the sides of the sample. As this negative charge builds a corresponding positive charge is set up on the opposite face of the sample from the stationary ion cores, thereby creating an electric field that points from positive to negative along the y-axis. This field will eventually become strong enough to keep further electrons from deflecting and a steady state condition will be achieved where the Lorentz force will be exactly balanced by the force of the induced electric field, or

$$
E_{y}=v_{x} B_{z} .
$$

The current density going into the sample is given by $\mathrm{J}_{\mathrm{x}}=\mathrm{nqv}_{\mathrm{x}}$, so we can write 


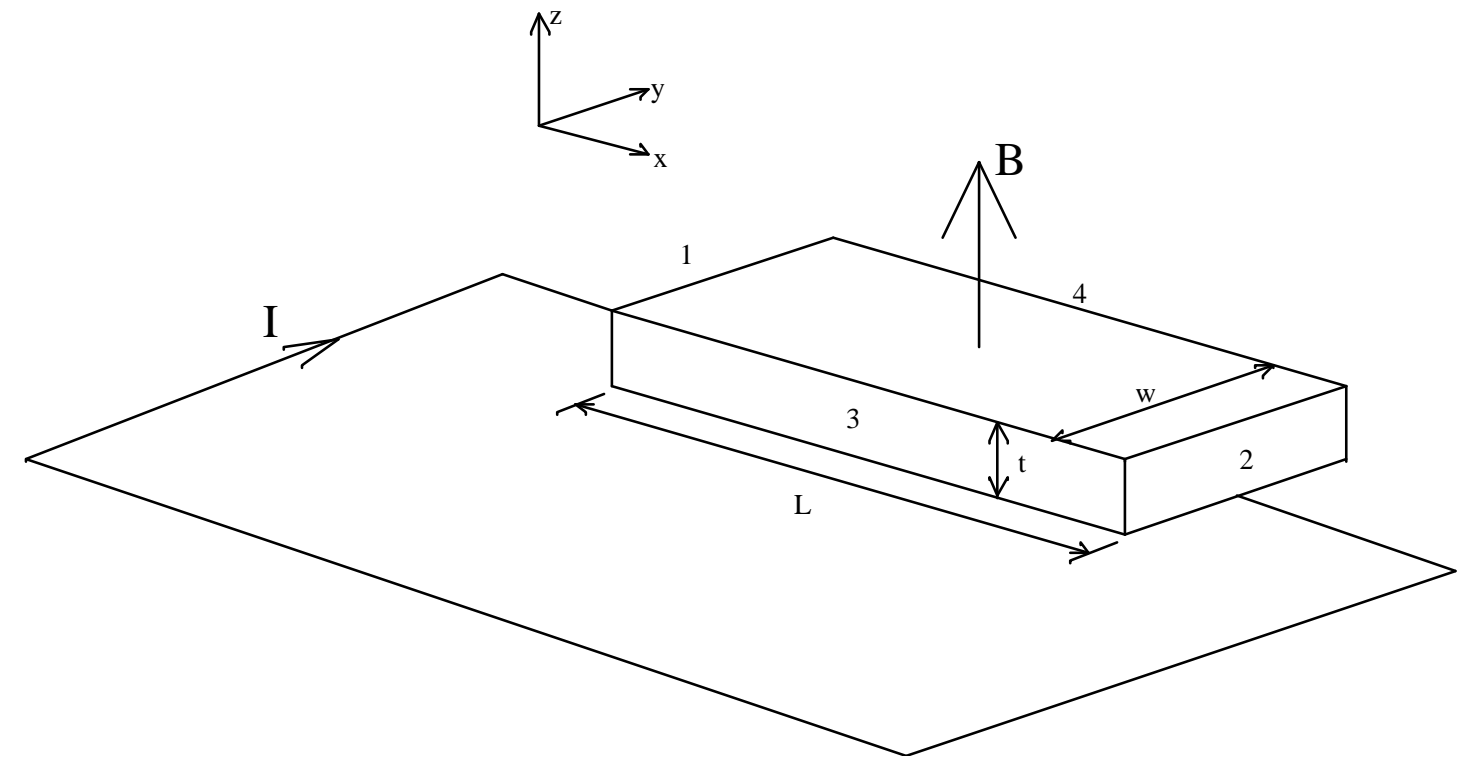

Figure B.1. Schematic for conventional Hall effect measurements. 


$$
E_{y}=\frac{J_{x} B_{z}}{n q}
$$

By considering the macroscopic quantities measured, it can be shown from this that the Hall coefficient, $\mathrm{R}_{\mathrm{H}}$, is simply

$$
R_{H}=\frac{1}{n q} .
$$

From the current density, and the relation that $\mathbf{J}=\sigma \mathbf{E}$, one can also show that the conductivity of the sample is given by

$$
\sigma=n q \mu
$$

where $\mu$ is the mobility of the electrons, and the velocity of the electrons is equal to their mobility multiplied by the electric field. From these two values it is possible to calculate the Hall mobility and carrier concentration of the sample.

$$
\begin{gathered}
\mu_{H}=\left|R_{H}\right| \sigma \\
n=\frac{1}{q R_{H}}
\end{gathered}
$$

The above equations can also be derived from a quantum mechanical approach for semiconductors that introduce a constant factor related to scattering lifetimes. This factor is of order unity, and is almost universally ignored in interpreting Hall results since it is extremely difficult to calculate. Also, if conduction is due to positive charges, the same procedure gives the results for p-type material where $\mathrm{n}$ is replaced by $\mathrm{p}$, the hole concentration. 
The technique developed by van der Pauw can be used for samples of arbitrary shape to yield $\mathrm{R}_{\mathrm{H}}$ and $\sigma$. The van der Pauw technique consists of placing an ohmic contact at four different points on a sample, usually the four corners of a square sample. This geometry allows measurements of samples of arbitrary shape so that no post growth processing is required. A weighting function is also used that corrects for differences in contact placement and sample shape. In addition, each measurement that depends on current flow is made using both positive and negative currents, effectively reversing the sign of the measured quantity. This allows for averaging to eliminate ohmic voltage drops during Hall measurements and to subtract out any voltages due to the contacts themselves. The van der Pauw measurement gives both sample conductivity, $\sigma$, and the Hall coefficient, $R_{H}$, determined from the Hall voltage as $\Delta V_{H}=R_{H} I B$ where $I$ is the current flowing in the sample and B is the applied magnetic field.

In order to interpret the Hall results to find the total numbers of acceptors and donors in a doped sample, we must also solve the charge balance equation.

$$
p-n=N_{a}-N_{d}+n_{d}-p_{a}
$$

where $\mathrm{N}_{\mathrm{a}}$ is the total number of acceptors per unit volume, $\mathrm{N}_{\mathrm{d}}$ is the total number of donors per unit volume, and $\mathrm{p}_{\mathrm{a}}$ and $\mathrm{n}_{\mathrm{d}}$ are the number of unionized acceptors and donors, respectively. The number of unionized acceptors, $\mathrm{p}_{\mathrm{a}}$, can be written in terms of $\mathrm{N}_{\mathrm{a}}$ as follows:

$$
p_{a}=\frac{N_{a}}{\frac{1}{g_{a}} e^{\frac{\left(\mu-E_{a}\right)}{k_{B} T}}+1}
$$

where $\mathrm{k}_{\mathrm{B}}$ is the Boltzmann constant, $\mathrm{E}_{\mathrm{a}}$ is the energy of the acceptor level as defined from the valence band, $\mu$ is the energy of the Fermi level, and $g_{a}$ is the degeneracy of the acceptor. If we assume complete ionization of the donors, valid for most p-type semiconductors, then 


$$
n_{d} \cong 0
$$

and the charge balance equation can be written

$$
p-n=N_{a}\left(1-\frac{1}{\frac{1}{g_{a}} e^{\frac{\left(\mu-E_{a}\right)}{k_{B} T}}+1}\right)-N_{d} .
$$

From here it is helpful to eliminate the Fermi energy from the equation. With the assumption that the electron concentration is negligible compared to the concentration of holes in the temperature region of interest, and

$$
p=P_{v}(T) e^{\frac{-\left(\mu-E_{v}\right)}{k_{B} T}}
$$

where $P_{v}(T)$ is the near gap density of hole states, one may solve for $e^{-\mu / k T}$. Thus, one may arrive at the useful form of the charge balance equation.

$p(T)=\frac{-P_{v} e^{\frac{-\left(E_{a}-E_{v}\right)}{k_{B} T}}}{2 g_{a}}-\frac{N_{d}}{2}+\sqrt{\frac{P_{v}^{2} e^{\frac{-2\left(E_{a}-E_{v}\right)}{k_{B} T}}}{4 g_{a}^{2}}+\frac{N_{d}^{2}}{4}+\frac{P_{v} N_{d} e^{\frac{-\left(E_{a}-E_{v}\right)}{k_{B} T}}}{2 g_{a}}+\frac{P_{v}\left(N_{a}-N_{d}\right) e^{\frac{-\left(E_{a}-E_{v}\right)}{k_{B} T}}}{g_{a}}}$

This is the equation used to fit the temperature-dependent carrier concentration data for the Hall samples. From the above equation it is possible to make simplifying assumptions which can lead to ambiguity in the acceptor energy, $\mathrm{E}_{\mathrm{a}}$. If you assume that $\mathrm{N}_{\mathrm{d}}<<\mathrm{p}<<\mathrm{N}_{\mathrm{a}}$ then it can be shown that

$$
p(T) \cong \sqrt{\frac{P_{v}\left(N_{a}-N_{d}\right)}{g_{a}}} e^{\frac{-\left(E_{a}-E_{v}\right)}{2 k_{B} T}} .
$$


However, if you assume that $\mathrm{p}<<\mathrm{N}_{\mathrm{d}}<<\mathrm{N}_{\mathrm{a}}$ then the equation reduces to

$$
p(T) \cong \frac{P_{v}\left(N_{a}-N_{d}\right)}{N_{d} g_{a}} e^{\frac{-\left(E_{a}-E_{v}\right)}{k_{B} T}} .
$$

Consequently, a factor of 2 difference can arise in the experimental determination of $\mathrm{E}_{\mathrm{a}}$ depending on which approximation is used and is often a source of confusion in Hall analysis. Indeed, an a priori assumption about doping must be made in order to choose which equation to use. No such possible discrepancy arises if the full charge balance equation is used.

Thus, a number of things can be determined about a sample from these Hall measurements. At low temperatures, where conduction due to intrinsic carriers is negligible, it is possible to determine the activation energy, $E_{a}$, the mobility of the holes, and the compensation ratio, defined as the number of donors to the number of acceptors. It is only possible to determine the ratio of donors to acceptors for relatively deep impurities such as $\mathrm{Mg}$ and $\mathrm{Be}$ in GaN and not absolute numbers for $\mathrm{N}_{\mathrm{a}}$ and $\mathrm{N}_{\mathrm{d}}$ because measurements are made in the regime of incomplete ionization corresponding to the second approximation above. It is possible with impurities that form shallow levels in $\mathrm{GaN}$, e.g. oxygen donors, to determine absolute quantities since essentially all of the oxygen will be ionized at room temperature. Hence, the carrier concentration at high temperatures will represent the value $\mathrm{N}_{\mathrm{d}}-\mathrm{N}_{\mathrm{a}}$ for oxygen-doped material. For deeper levels, this is true only when high enough temperatures to completely ionize the acceptors are used, invoking a need for the complete expression to determine the value for $N_{a}-N_{d}$ unambiguously. 


\section{Appendix C: $\quad$ Elements Measured by SIMS ${ }^{117}$}

\begin{tabular}{|c|c|c|c|}
\hline Element & Precision ${ }^{(\mathrm{a}}$ & $\begin{array}{l}\text { Absolute } \\
\text { Accuracy }\end{array}$ & $\begin{array}{l}\text { Minimum Detectable } \\
\text { Level in } \mathrm{GaN}\left(\mathrm{cm}^{-3}\right)\end{array}$ \\
\hline Hydrogen & \multirow{7}{*}{ $\pm 20 \%$} & \multirow{7}{*}{ $\pm 30 \%$} & $1 \times 10^{17}$ \\
\hline Beryllium & & & $1 \times 10^{14}$ \\
\hline Boron & & & $6 \times 10^{14}$ \\
\hline Carbon & & & $1 \times 10^{16}$ \\
\hline Oxygen & & & $1 \times 10^{16}$ \\
\hline Magnesium & & & $6 \times 10^{14}$ \\
\hline Silicon & & & $1 \times 10^{15}$ \\
\hline
\end{tabular}

a) Precision is the sample-to-sample measurement variation that is dependent on the impurity matrix combination and the analysis protocol.

b) Absolute accuracy is based on the accuracy of the ion-implanted reference standard used for quantification. 


\section{References}

1. W.C. Johnson, J.B. Parsons, and M.C. Crew, J. Phys. Chem. 36, 2561 (1932).

2. E.H.C. Parker, editor, The Technology and Physics of Molecular Beam Epitaxy, New York: Plenum Press, 1985.

3. R.L. Gunshore, L.A. Kolodziejski, A.V. Nurmikko, and N. Otsuka, in Semiconductors and Semimetals, vol. 33, edited by T. Pearsal, New York: Academic Press, 1990.

4. S.J. Pearton, J.C. Zolper, R.J. Shul, and F. Ren, J. Appl. Phys. 86, 1 (1999).

5. S. Strite and H. Morkoc, J. Vac. Sci. Technol. B 10, 1237 (1992), and references therein.

6. H. Morkoc, S. Strite, G.B. Gao, M.E. Lin, B. Sverdlov, and M. Burns, J. Appl. Phys. 76, 1363 (1994).

7. J.I. Pankove, E.A. Miller, and J.E. Berkeyheiser, RCA Review 32, 283 (1971).

8. B.J. Baliga, IEEE Electron. Device Lett. 10, 455 (1989).

9. T.P. Chow and R. Tyagi, IEEE Trans. Electron. Devices 41, 1481 (1994).

10. R.J. Trew, M.W. Shin, and V. Gatto, Solid State Electron. 41, 1561 (1997).

11. J.B. Webb, H. Tang, S. Rolfe, and J.A. Bardwell, Appl. Phys. Lett. 75, 953 (1999).

12. H. Amano, I. Akasaki, T. Kozawa, K. Hiramatsu, N. Sawaki, and K. Ikeda, J. Lumin. 40/41, 121 (1988).

13. W. Gotz, N.M. Johnson, J. Walker, D.P. Bour, and R.A. Street, Appl. Phys. Lett. 68, 667 (1996).

14. P. Kozodoy, H. Xing, S.P. DenBaars, U.K. Mishra, A. Saxler, R. Perrin, S. Elhamri, and W.C. Mitchel, J. Appl. Phys. 87, 1832 (2000).

15. H. Amano, M. Kito, K. Hiramatsu, and I. Akasaki, Jpn. J. Appl. Phys 28, L2112 (1989).

16. S. Nakamura, N. Iwasa, M. Seno, and T. Mukai, Jpn. J. Appl. Phys. 31, 1258 (1992).

17. K. Iwata, H. Asahi, S.J. Yu, K. Asami, H. Fujita, M. Fushida, and S. Gonda, Jpn. J. Appl. Phys. 35, L289 (1996). 
18. P. Hacke, G. Feuillet, H. Okumura, and S. Yoshida, Appl. Phys. Lett. 69, 2507 (1996).

19. A.R. Smith, V. Ramachandran, R.M. Feenstra, D.W. Greve, A. Ptak, T. Myers, W. Sarney, L. Salamanca-Riba, M. Shin, and M. Skowronski, MRS Internet J. Nitride Semicond. Res. 3, 12 (1998).

20. M.R. Millecchia, Masters Thesis, West Virginia University (1997).

21. Applied EPI, Inc. MBE Products Group Application Note, January (1996).

22. T.A. Flaim and P.D. Ownby, J. Vac. Sci. Technol. 8, 661 (1971).

23. E.J. Tarsa, B. Heying, X.H. Wu, P. Fini, S.P. DenBaars, and J.S. Speck, J. Appl. Phys. 82, 5472 (1997).

24. R. Held, D.E. Crawford, A.M. Johnson, A.M. Dabiran, and P.I. Cohen, J. Electron. Mater. 26, 272 (1997).

25. K.R. Evans, R. Kaspi, C.R. Jones, R.E. Sherriff, V. Jogai, and D.C. Reynolds, J. Cryst. Growth 127, 523 (1993).

26. C.R. Jones, T. Lei, R. Kaspi, and K.R. Evans, Mat. Res. Soc. Symp. Proc. 395, 141 (1996).

27. R. Held, D.E. Crawford, A.M. Johnston, A.M. Dabiran, and P.I. Cohen, J. Electron. Mater. 26, 272 (1997).

28. L.J. van der Pauw, Philips Res. Rpts. 13, 1 (1958).

29. V.Yu. Davydov, N.S. Averkiev, I.N. Goncharuk, D.K. Nelson, I.P. Nikitina, A.S. Polkovnikov, A.N. Smirnov, and M.A. Jacobson, J. Appl. Phys. 82, 5097 (1997).

30. S. Ruvimov, Z. Liliental-Weber, T. Suski, J.W. Ager III, J. Washburn, J. Krueger, C. Kisielowski, E.R. Weber, H. Amano, and I. Akasaki, Appl. Phys. Lett. 69, 990 (1996).

31. P.M. Asbeck, E.T. Yu, S.S. Lau, G.J. Sullivan, J. Van Hove, and J. Redwing, Electron. Lett. 33, 1230 (1997).

32. I.P. Smorchkova, C.R. Elsass, J.P. Ibbetson, R. Vetury, B. Heying, P. Fini, E. Haus, S.P. DenBaars, J.S. Speck, and U.K. Mishra, J. Appl. Phys. 86, 4520 (1999). 
33. A.D. Bykhovski, B.L. Gelmont, and M.S. Shur, J. Appl. Phys. 81, 6332 (1997).

34. X.Q. Shen, T. Ide, S.H. Cho, M. Shimizu, S. Hara, H. Okumura, S. Sonoda, and S. Shimizu, J. Cryst. Growth 218, 155 (2000).

35. T. Ide, M. Shimizu, X.Q. Shen, S. Hara, H. Okumura, and T. Nemoto, Mat. Sci. Forum 338, 1459 (2000).

36. R. Held, D.E. Crawford, A.M. Johnson, A.M. Dabiran, and P.I. Cohen, Surf. Rev. Lett. 5, 913 (1998).

37. A.R. Smith, V. Ramachandran, R.M. Feenstra, D.W. Greve, M.S. Shin, M. Skowronski, J. Neugebauer, and J.E. Northrup, J. Vac. Sci. Technol. A 16, 1641 (1998).

38. T. Zywietz, J. Neugebauer, and M. Scheffler, Appl. Phys. Lett. 73, 487 (1998).

39. A. Lofthus and P.H. Krupenie, J. Chem. Phys. 6, No. 1 (1977).

40. R. Beresford, K.S. Stevens, Q. Cui, A. Schwartzman, H. Cheng, Mat. Res. Soc. Symp. Proc. 449, 361 (1997).

41. S.L. Buczkowski, Masters Thesis, West Virginia University (1996).

42. S. Kumar, Masters Thesis, West Virginia University (1996).

43. I. Berishev, E. Kim, and A. Bensaoula, J. Vac. Sci. Technol. A16, 2791 (1998).

44. M.A.L. Johnson, Zonghai Yu, C. Boney, W.C. Hughes, J.W. Cook, Jr., J.F. Schetzina, H. Zho, B.J. Skromme, and J.A. Redmond, Mater. Res. Soc. Symp. Proc. 449, 215 (1997).

45. M.A.L. Johnson, J.D. Brown, N.A. El-Masry, J.W. Cook, Jr., J.F. Schetzina, H.S. Kong, and J.A. Redmond, J. Vac. Sci. Technol. B 16, 1282 (1998).

46. N. Grandjean, J. Massies, and L. Leroux, Appl. Phys. Lett. 69, 2071 (1996).

47. T.D. Moustakas, R.J. Molnar, T. Lei, G. Menon, and J.C.R. Eddy, Mater. Res. Soc. Symp. Proc. 242, 427 (1992).

48. F. Widmann, G. Feuillet, B. Daudin, and J.L. Rouviere, J. Appl. Phys. 85, 1550 (1999). 
49. M. Yeadon, M.T. Marshall, F. Hamdani, S. Pekin, H. Morkoc, and J.M. Gibson, J. Appl. Phys. 83, 2847 (1998).

50. A.J. Ptak, K.S. Ziemer, L.J. Holbert, C.D. Stinespring, and T.H. Myers, MRS Internet J. Nitride Semicond. Res. 5S1, W3.33 (2000). Also appears in Mat. Res. Soc. Symp. Proc. 595, W3.33.1, (1999).

51. Handbook of Auger Electron Spectroscopy, Perkin-Elmer, Physical Electronics Division (Eden Prairie, MN, 1995).

52. Analysis followed standard procedures such as outlined in D. Briggs and M.P. Seah, Practical Surface Analysis Vol. 1, John Wiley and Sons (Chichester, England, 1990), p. 207.

53. S. Tanuma, C.J. Powell, and D.R. Penn, Surf. Interface Anal., 17911 (1991).

54. M. Moldovan, L.S. Hirsch, A.J. Ptak, C.D. Stinespring, T.H. Myers, and N.C. Giles, J. Elec. Mat, 27, 756 (1998).

55. A.J. Ptak, K.S. Ziemer, M.R. Millecchia, C.D. Stinespring, and T.H. Myers, MRS Internet J. Nitride Semicond. Res. 4S1, G3.10 (1999).

56. A.Y. Polyakov, M. Shin, W. Qian, M. Skowronski, D.W. Greve and R.G. Wilson, J. Appl. Phys 81, 1715 (1997).

57. T.H. Myers, L.S. Hirsch, L.T. Romano, and M.R. Richards-Babb, J. Vac. Sci. Technol. B, 16, 2261 (1998).

58. D.D. Koleske, A.E. Wickenden, R.L. Henry, W.J. DeSisto, and R.J. Gorman, J. Appl. Phys. 84, 1998 (1998).

59. R. Groh, G. Gerey, L. Bartha, and J.I. Pankove, Phys. Status Soldi A 26, 353 (1974).

60. O. Brandt, H. Yang, and K.H. Ploog, Phys. Rev. B 54, 4432 (1996).

61. Zonghai Yu, S.L. Buczkowski, N.C. Giles, T.H. Myers, and M.R. Richards-Babb, Appl. Phys. Lett. 69, 2731 (1996).

62. N.-E. Lee, R.C. Powell, Y.-W. Kim, and J.E. Greene, J. Vac. Sci. Technol. A 13, $2293(1995)$. 
63. N. Newman, J. Cryst. Growth 178, 102 (1997); N. Newman, "Thermochemistry of III-N Semiconductors" in Semiconductors and Semimetals 50, pp. 55-101 (Academic Press Inc, 1998).

64. Private communication, R.P. Muller, Beckman Institute, Caltech, Pasadena, CA.

65. J.M. Reifsnider, D.W. Gotthold, A.L. Holmes, and B.G. Streetman, J. Vac. Sci. Technol. B 16, 1278 (1998).

66. Private communication, J.S. Speck, Materials Department, University of California Santa Barbara, Santa Barbara, CA.

67. A.R. Smith, R.M. Feenstra, D.W. Greve, J. Neugebauer, and J.E. Northrup, Phys. Rev. Lett. 79, 3934 (1997).

68. L.T. Romano and T.H. Myers, Appl. Phys. Lett. 71, 3486 (1997).

69. D.D. Koleske, A.E. Wickenden, R.L. Henry, M.E. Twigg, J.C. Culbertson, and R.J. Gorman, Appl. Phys. Lett. 73, 2018 (1998).

70. M.E. Bartram, MRS Internet J. Nitride Semicond. Res. 4S1, G3.68 (1999).

71. H. Amano, M. Kito, K. Hiramatsu, and I. Akasaki, Jpn. J. Appl. Phys., Part 228 , L2112 (1989).

72. S. Nakamura, T. Mukai, and M. Senoh, Jpn. J. Appl. Phys., Part 2 30, L1998 (1991).

73. I.P. Smorchkova, E. Haus, B. Heying, P. Kozodoy, P. Fini, J.P. Ibbetson, S. Keller, S.P. DenBaars, J.S. Speck, and U.K. Mishra, Appl. Phys. Lett. 76, 718 (2000).

74. L.K. Li, M.J. Jurkovic, W.I. Wang, J.M. Van Hove, and P.P. Chow, Appl. Phys. Lett. 76, 1740 (2000).

75. J.W. Orton, C.T. Foxon, T.S. Cheng, S.E. Hooper, S.V. Novikov, B. Ya. Ber, and Yu. A. Kudriavtsev, J. Cryst. Growth 197, 7 (1999).

76. S. Guha, N.A. Bojarczuk, and F. Cardone, Appl. Phys. Lett. 71, 1685 (1997).

77. T.S. Cheng, S.V. Novikov, C.T. Foxon, and J.W. Orton, Solid State Comm. 109, 439 (1999).

78. V. Ramachandran, R.M. Feenstra, J.E. Northrup, and D.W. Greve, MRS Internet J. Nitride Semicond. Res. 5S1, W3.65 (2000). 
79. V. Ramachandran, R.M. Feenstra, W.L. Sarney, L. Salamanca-Riba, J.E. Northrup, L.T. Romano, and D. W. Greve, Appl. Phys. Lett. 75, 808 (1999).

80. J. Neugebauer and C.G. Van de Walle, Phys. Rev. Lett. 75, 4452 (1995).

81. C. Bungaro, K. Rapcewicz, and J. Bernholc, Phys. Rev. B59, 9771 (1999).

82. J. Neugebauer, T. Zywietz, M. Scheffler, and J.E. Northrup, Proc. $2^{\text {nd }}$ Int. Conf. on Nitride Semiconductors, Tokushima, Japan, 216 (1997).

83. L.T. Romano, J.E. Northrup, A.J. Ptak, and T.H. Myers, Appl. Phys. Lett. 77, 2479 (2000).

84. B.J. Skromme and G. L. Martinez, Mat. Res. Soc. Symp. Proc. 595, W9.8.1, (1999).

85. B.J. Skromme, H. Zhao, B. Goldenberg, H.S. Kong, M.T. Leonard, G.E. Bulman, C.R. Abernathy, and S.J. Pearton, Mater. Res. Soc. Symp. Proc. 449, 713 (1996).

86. F. Bernardini, V. Fiorentini, and A. Bosin, Appl. Phys. Lett 70, 2990 (1997).

87. M.A. Sanchez-Garcia, E. Calleja, F.J. Sanchez, F. Calle, E. Monroy, D. Basak, E. Munoz, C. Villar, A. Sanz-Hervas, M. Aguilar, J.J. Serrano, and J.M. Blanco, J. Electron. Mater. 27, 276 (1998).

88. D.J. Dewsnip, A.V. Andrianov, I. Harrison, J.W. Orton, D.E. Lacklison, G.B. Ren, S.E. Hooper, T.S. Cheng, and C.T. Foxon, Semicond. Sci. Technol. 13, 500 (1998). 89. T.H. Myers, A.J. Ptak, Lijun Wang, and N.C. Giles, Institute of Pure and Applied Physics Conference Series 1, 451 (2000).

90. K.H. Ploog and O. Brandt, J. Vac. Sci. Technol. A 16, 1609 (1998).

91. Y. Sun, L.S. Tan, S.J. Chua, and S. Prakash, Mat. Res. Soc. Symp. Proc. 595, W3.82.1 (2000).

92. C.G. Van de Walle, Report for the Office of Naval Research, Contract No. N0001499-C-0161.

93. Private communication, C.G. Van de Walle, Xerox Palo Alto Research Center, Palo Alto, CA.

94. B.L. VanMil, A.J. Ptak, N.C. Giles, T.H. Myers, P.J. Treado, M.P. Nelson, J.M. Ribar, and R.D. Smith, J. Vac. Sci. Technol. B 18, 2295 (2000). 
95. H.H. Farrell, J. L. de Miguel, and M.C. Tamargo, J. Appl. Phys 65, 4084 (1989).

96. Y.S. Wu, C.R. Becker, A. Wang, R.N. Bicknell-Tassius, and G. Landwehr, J. Appl. Phys. 69, 268 (1991).

97. P. Vennegues, M. Benaissa, B. Beaumont, E. Feltin, P. De-Mierry, S. Dalmasso, M. Leroux, and P. Gibart, Appl. Phys. Lett. 77, 880 (2000).

98. F.J. Sanchez, F. Calle, M.A. Sanchez-Garcia, E. Calleja, E. Munoz, C.H. Molloy, D.J. Somerford, J.J. Serrano, and J.M. Blanco, Semicond. Sci. Technol. 13, 1130 (1998).

99. A. Salvador, W. Kim, O. Atkas, A. Botchkarev, Z. Fan, and H. Morkoc, Appl. Phys. Lett. 69, 2692(1996).

100. T.K. Zywietz, J. Neugebauer, and M. Scheffler, Appl. Phys. Lett. 74, 1695 (1999).

101. M. Sumiya, K. Yoshimura, K. Ohtsuka, and S. Fuke, Appl. Phys. Lett. 76, 2098 (2000).

102. C.G. Van de Walle and J. Neugebauer, Mat. Sci. Forum 258, 19 (1997).

103. A.R. Smith, R.M. Feenstra, D.W. Greve, M.S. Shin, M. Skowronski, J. Neugebauer, and J.E. Northrup, J. Vac. Sci. Technol. B 16, 2242 (1998).

104. See, for example, R.H. Bube, Electronic Properties of Crystalline Solids: An Introduction to Fundamentals, (Academic Press, Inc., New York, New York, 1974).

105. D. Meister, M. Bohm, M. Topf, W. Kriegseis, W. Burkhardt, I. Dirnstorfer, S. Rosel, B. Farangis, B.K. Meyer, A. Hoffmann, H. Siegle, C. Thomsen, J. Christen, and F. Bertram, J. Appl. Phys. 88, 1811 (2000).

106. See, for example, S.N. Mohammad, A.A. Salvador, and H. Morkoc, Proc. of the IEEE 83, 1306 (1995).

107. J. Neugebauer and C.G. Van de Walle, Appl. Phys. Lett. 69, 503 (1996).

108. J. Oila, V. Ranki, J. Kivioja, K. Saarinen, P. Hautojarvi, J. Likonen, J.M. Baranowski, K. Pakula, T. Suski, M. Leszczynski, and I. Grzegory, Phys. Rev. B 63, 04205 (2001).

109. H.C. Yang, T.Y. Lin, and Y.F. Chen, Phys. Rev. B 62, 12593 (2000). 
110. L.T. Romano, C.G. Van de Walle, J.W. Ager III, W. Gotz, and R.S. Kern, J. Appl. Phys. 87, 7745 (2000).

111. O. Brandt, H. Yang, H. Kostial, and K.H. Ploog, Appl. Phys. Lett. 69, 2707 (1996).

112. S. Fuke, H. Teshigawara, K. Kuwahara, Y. Takano, T. Ito, M. Yanagihara, and K. Ohtsuka, J. Appl. Phys. 83, 764 (1997).

113. C.-C. Yang, M.-C. Wu, C-A. Chang, and G-C. Chi, J. Appl. Phys. 85, 8427 (1999).

114. K.S. Kim, C.S. Oh, K.J. Lee, G.M. Yang, C-H. Hong, K.Y. Lim, H.J. Lee, and A. Yoshikawa, J. Appl. Phys. 85, 8441 (1999).

115. C-C. Yang, M-C. Wu, and G-C. Chi, J. Appl. Phys. 86, 6120 (1999).

116. See, for example, R.C. Powell, N.-E. Lee, Y.-W. Kim, and J.E. Greene, J. Appl. Phys. 73, 189 (1993).

117. Evans Analytical Group, Charles Evans and Associates SIMS Analysis Report (2000). 